\title{
Characterization of LIN-61 methyl mark binding and its function in $C$. elegans vulva development
}

\author{
Dissertation \\ for the award of the degree \\ 'Doctor rerum naturalium' (Dr. rer. nat.) \\ Division of Mathematics and Natural Sciences \\ of the Georg-August-University Göttingen
}

submitted by

Nora Köster-Eiserfunke

born in Aachen

May 2010 
Thesis Supervisor:

Dr. Wolfgang Fischle

Doctoral Committee:

Dr. Wolfgang Fischle ( $1^{\text {st }}$ Referee) Chromatin Biochemistry,

Max-Planck-Institute for biophysical

Chemistry, Göttingen

Prof. Dr. Herbert Jäckle (2 ${ }^{\text {nd }}$ Referee) Molecular developmental Biology,

Max-Planck-Institute for biophysical

Chemistry, Göttingen

Prof. Dr. Sigrid Hoyer-Fender

Developmental Biology, Georg-August-

University, Göttingen

Date of submission of thesis: 31.05 .2010

Date of oral exam: 02.08.2010 


\begin{abstract}
Affidavit
I hereby ensure that the presented thesis "Characterization of LIN-61 methyl mark binding and its function in $C$. elegans vulva development" has been written independently and with no other sources and aids than quoted.
\end{abstract}

May 31, 2010, Göttingen 


\section{Table of contents}

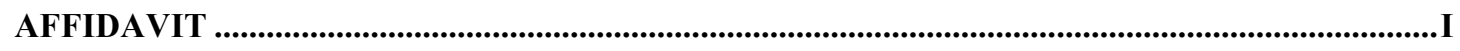

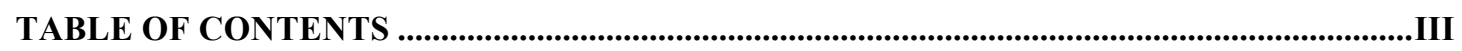

LIST OF FIGURES ................................................................................................................ VII

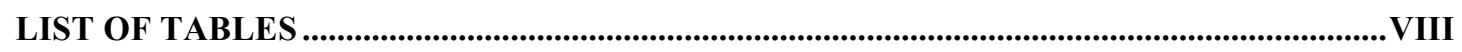

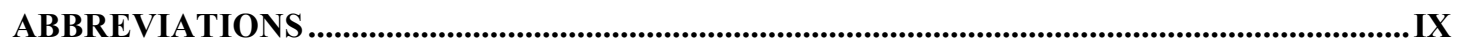

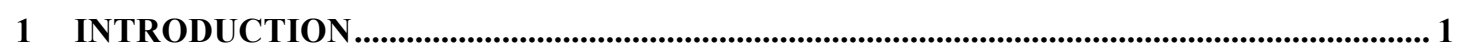

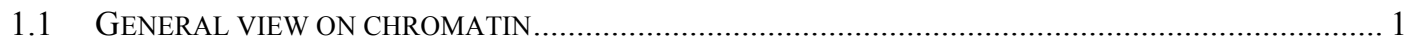

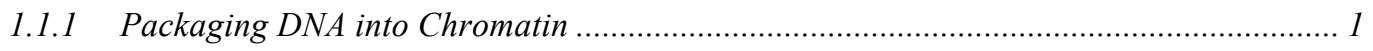

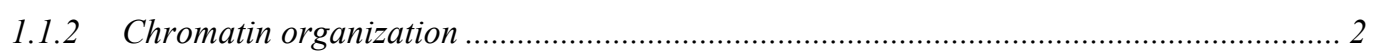

1.1.3 Specification and regulation of chromatin states ..................................................... 3

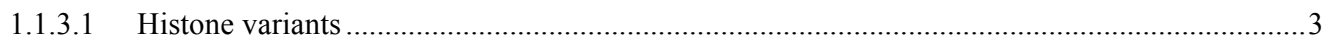

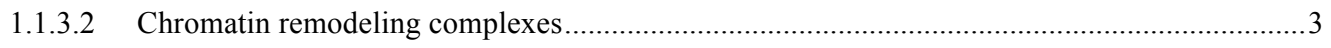

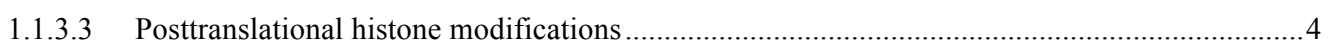

1.1.3.4 Chromatin effector proteins ..........................................................................................

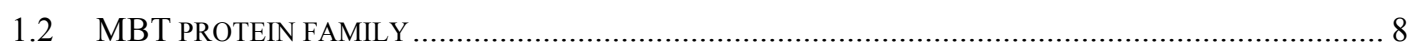

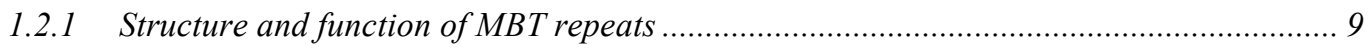

1.2.1.1 Binding of MBT repeats to methyl lysine ...........................................................................

1.2.1.2 Composition of methyl-lysine binding pockets within MBT proteins ..................................... 10

1.2.1.3 Multi domain organization of MBT repeats ........................................................................ 11

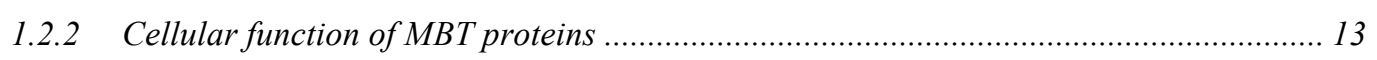

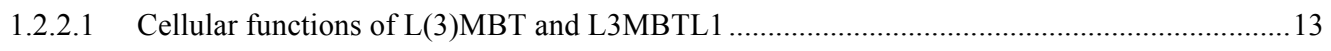

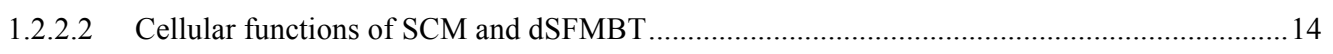

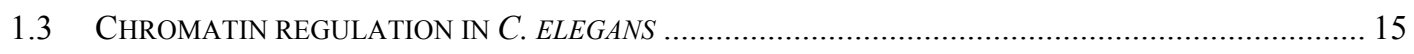

1.3.1 synMuvB pathway in C. elegans............................................................................... 16

1.3.1.1 Vulva development in C. elegans ..................................................................................... 16

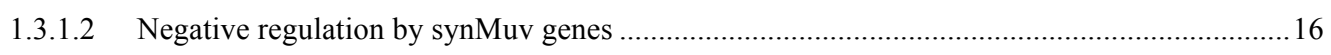

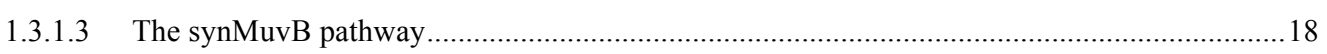

1.3.1.4 Biological function of synMuvB factors apart from vulval development.................................21

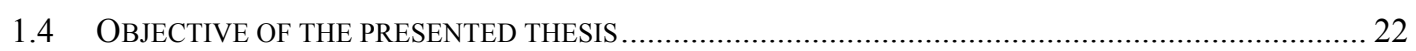

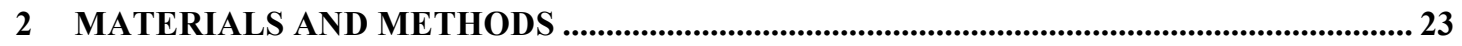

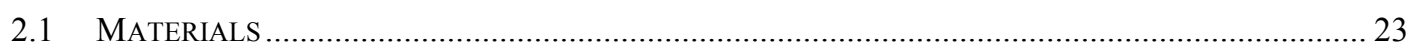

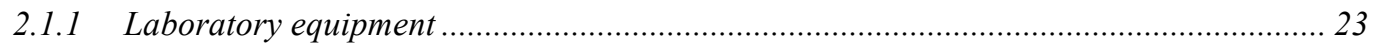

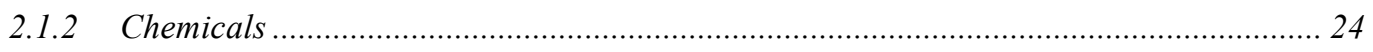

2.1.3 Consumables............................................................................................................. 25

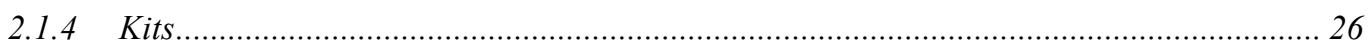

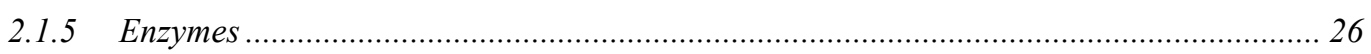




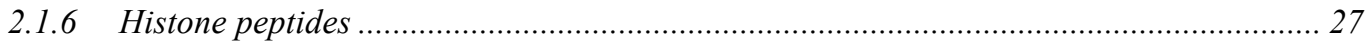

2.1.7 Antibodies obtained from different sources......................................................... 27

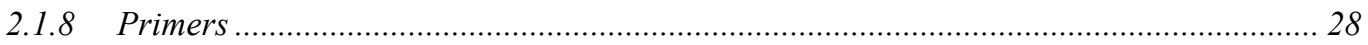

2.1.9 Plasmids obtained from different sources .......................................................... 28

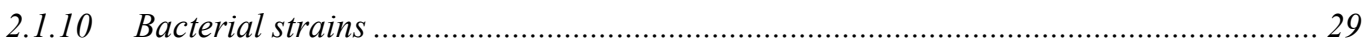

2.1.11 C. elegans strains obtained from different sources ..................................................... 29

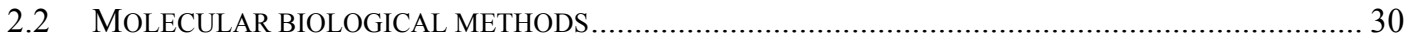

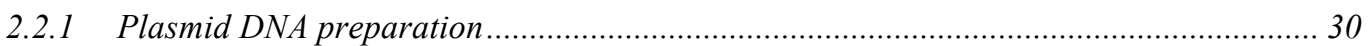

2.2.2 DNA digestion with restriction endonucleases............................................................. 30

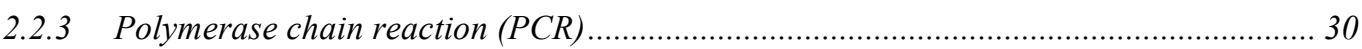

2.2.4 Separation and isolation of DNA fragments ............................................................... 31

2.2.5 Transformation of plasmids into chemically competent bacteria ................................ 32

2.2.6 General cloning procedure ........................................................................................... 32

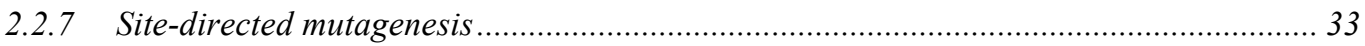

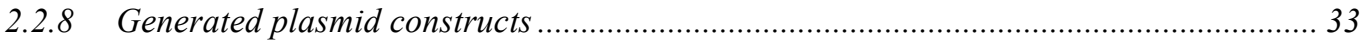

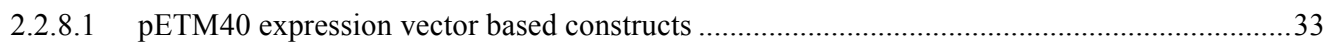

2.2.8.2 pcDNA3.1(+) mammalian expression vector based constructs ................................................33

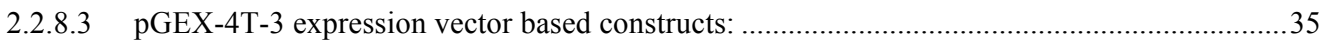

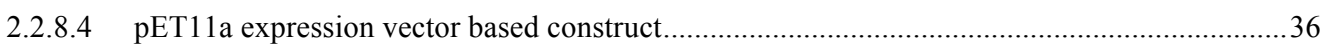

2.2.8.5 pET16b expression vector based construct ............................................................................. 36

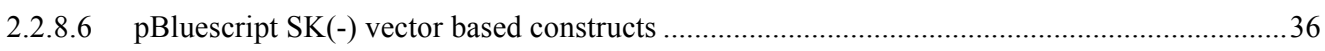

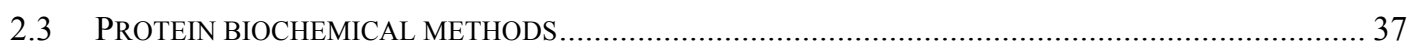

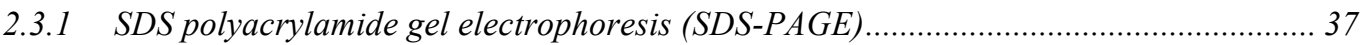

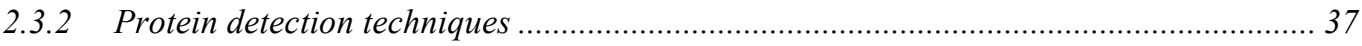

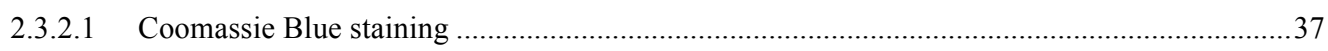

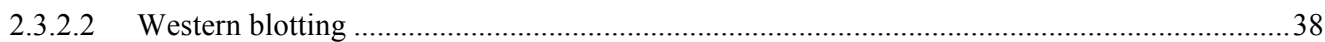

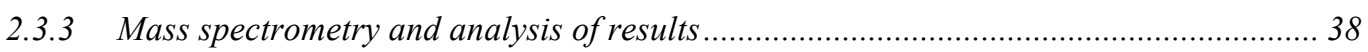

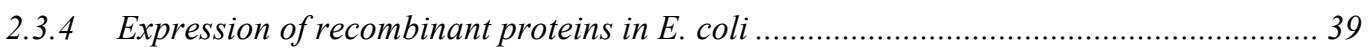

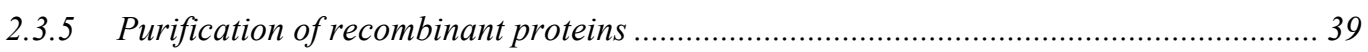

2.3.5.1 Purification of $\mathrm{HIS}_{6}$-tagged recombinant proteins ............................................................40

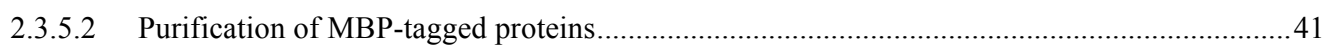

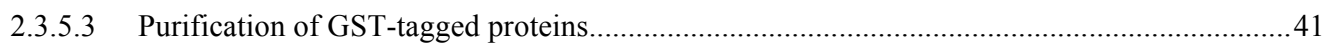

2.3.6 Expression of MYC-tagged recombinant proteins in rabbit reticulocyte lysate ............. 42

2.3.7 Histone peptide pull-down experiments ............................................................... 42

2.4 GENERATION OF LIN-61 SPECIFIC ANTIBODIES................................................................... 43

2.4.1 Generation of $\alpha L I N-61$ peptide antibodies ............................................................. 43

2.4.2 Generation of antibodies using recombinant LIN-61 for immunization ........................ 43

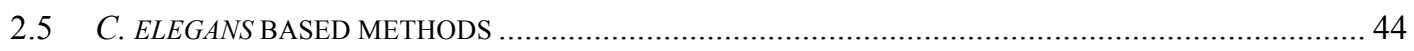

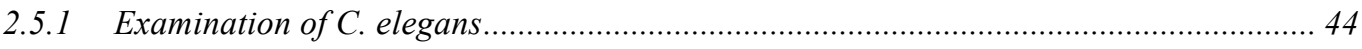

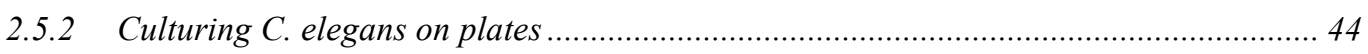

2.5.3 Culturing C. elegans in liquid medium......................................................................... 45 


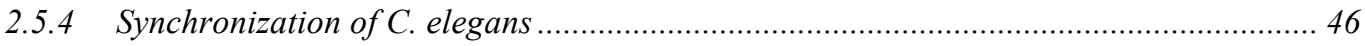

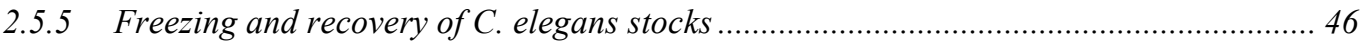

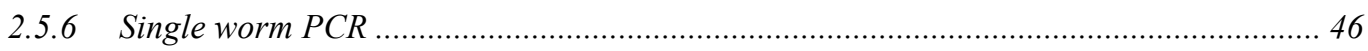

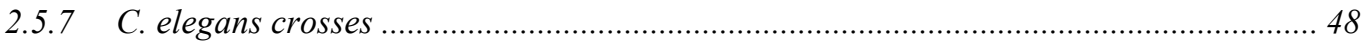

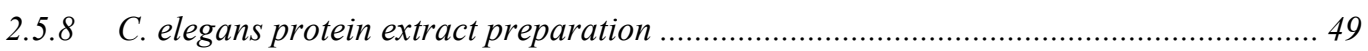

2.5.8.1 Protein extract preparation based on Cheeseman et al. (2004)..................................................49

2.5.8.2 Protein extract preparation based on Wysocka et al. (2006) .....................................................49

2.5.8.3 Generation of crude C. elegans lysate for western blot analysis.............................................50

2.5.9 Histone peptide pull-down experiments using C. elegans extract................................. 50

2.5.10 Gel filtration of C. elegans extract ................................................................. 50

2.5.11 Immunprecipitation of $C$. elegans extract ............................................................... 51

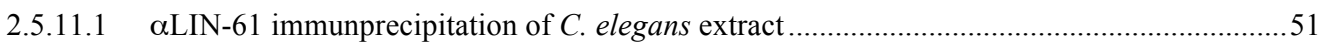

2.5.11.2 $\alpha \mathrm{GFP}$ immunprecipitation of $C$. elegans extract...................................................................51

2.5.12 C. elegans RNA isolation and Reverse Transcription ............................................. 52

2.5.13 Quantitative PCR analysis of lin-3 transcripts .................................................... 52

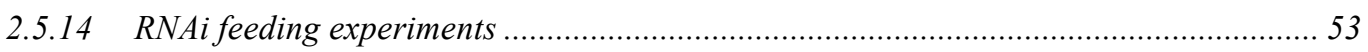

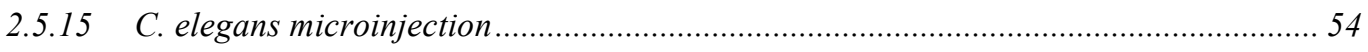

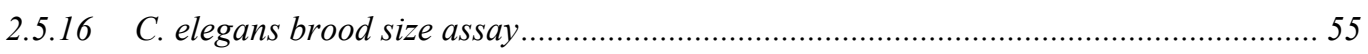

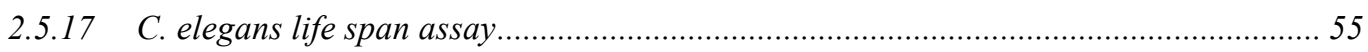

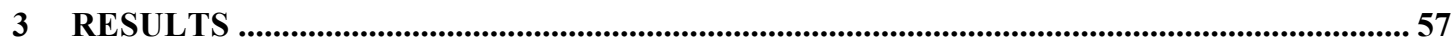

3.1 IDENTIFICATION OF H3K9ME3 BINDING PROTEINS IN C. ELEGANS ......................................... 57

3.1.1 The MBT proteins MBTR-1 and LIN-61 interact with H3K9me3 .............................. 57

3.2 CHARACTERIZATION OF LIN-61 H3K9ME3 INTERACTION...................................................... 58

3.2.1 Binding to H3K9me3 is an autonomous function of $L I N-61$...................................... 59

3.2.2 LIN-61 binds specifically to H3K9 methylated peptides ........................................... 63

3.2.3 Three MBT repeats and a functional aromatic cage motif are necessary for LIN-

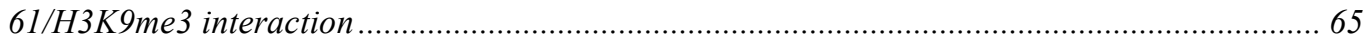

3.2.3.1 MBT repeats two to four of LIN-61 are necessary for H3K9me3 interaction .........................65

3.2.3.2 A functional aromatic cage motif is necessary for LIN-61 H3K9me3 binding .......................66

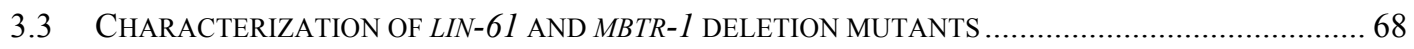

3.3.1 Description of lin-61 and mbtr-1 alleles used in this study ........................................ 68

3.3.2 lin-61 and mbtr-1 single and double mutants show no apparent morphological changes 70

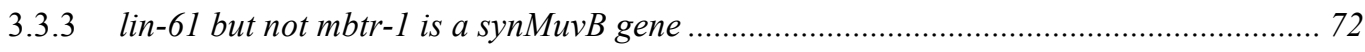

3.3.4 Genetic interaction of lin-61 with met-2 and hpl-2 causes a synMuv phenotype ........... 74

3.3.5 Synergistic effects of lin-61 with met-2 and hpl-2 on fertility .................................... 77

3.4 FUNCTION OF LIN-61 WITHIN THE SYNMUVB PATHWAY ....................................................... 82

3.4.1 LIN-61 H3K9me3 binding is essential for LIN-61 function within the synMuvB pathway 82 
3.4.1.1 Particular lin-61 alleles that cause a synMuv phenotype in a synMuvA mutant background

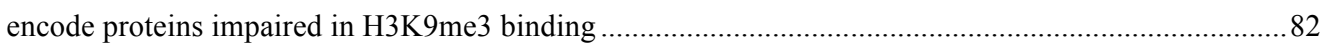

3.4.1.2 LIN-61 defective in H3K9me3 binding is not functional in the synMuvB pathway ................84

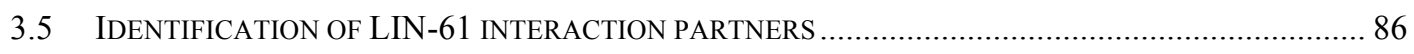

3.5.1 Identification of LIN-13 as interaction partner of LIN-61 ........................................ 86

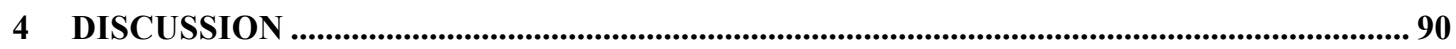

4.1 C. ELEGANS MBT PROTEINS AND HPL-2 INTERACT WITH H3K9ME3 ................................... 90

4.2 MBT PROTEINS IN C. ELEGANS DEFINE A NEW CLASS OF MBT PROTEINS ............................... 90

4.2.1 LIN-61 and MBTR-1 are special MBT proteins ................................................... 90

4.2.2 LIN-61 selectively binds to lysine in higher methylation state ................................... 92

4.3 BIOLOGICAL FUNCTIONS OF C. ELEGANS MBT PROTEINS........................................................ 95

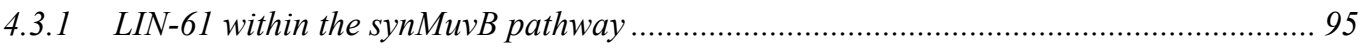

4.3.1.1 LIN-61 acts within the synMuvB pathway via H3K9 methyl mark binding ..........................95

4.3.1.2 LIN-61 interacts with the synMuvB factor LIN-13 ............................................................97

4.3.1.3 lin-61 genetically interacts with $\mathrm{hpl}-2$ and $m e t-2$..............................................................98

4.3.2 LIN-61 possess unique and redundant functions with HPL-2 and MET-2 besides the

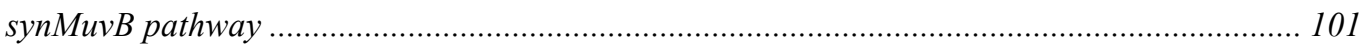

4.3.3 No biological functions are yet described for MBTR-1........................................ 104

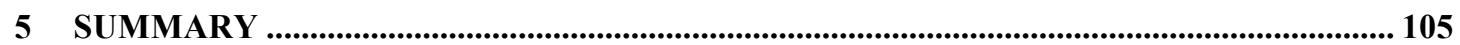

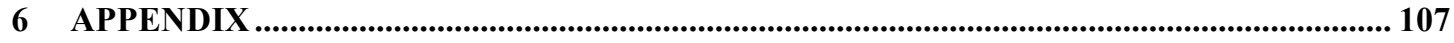

6.1 R PROGRAMMING FOR MASS SPECTROMETRY ANALYSIS ................................................. 107

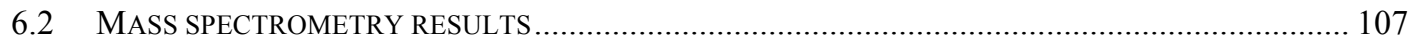

6.2.1 H3K9me3 bound C. elegans protein fraction ..................................................... 107

6.2.2 Common factors in bound fraction of H3K9me3 PD and two $\alpha L I N-61$ IPs from N2 C.

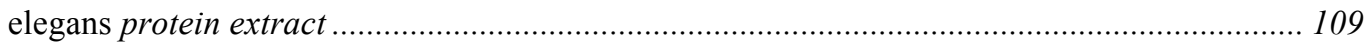

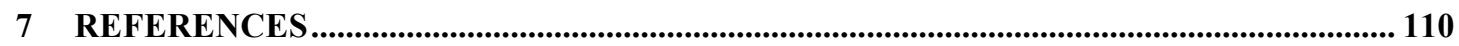

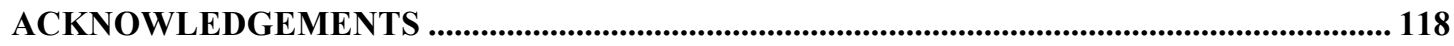

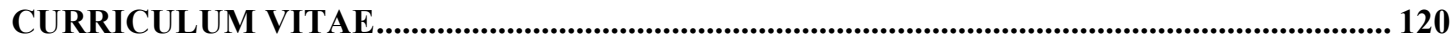




\section{List of figures}

FIGURE 1-1: SCHEMATIC OVERVIEW OF HISTONE MODIFICATIONS ACROSS A TYPICAL MAMMALIAN

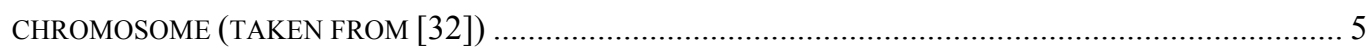

FIGURE 1-2: SCHEMATIC REPRESENTATION AND DOMAIN ORGANIZATION OF MBT PROTEINS................... 9

FIGURE 1-3: AROMATIC CAGE MOTIF OF L3MBTL1 AND RIBBON DIAGRAMS OF SELECTED MBT REPEATS 10

FIGURE 1-4: MODEL FOR THE MOLECULAR BASIS OF HOW SYNMUVA AND SYNMUVB GENES REGULATE ECTOPIC VULVAL INDUCTION 17

FigURE 2-1: SCHEMATIC OVERVIEW OF STUI - SACII FRAGMENT OF R06C7 ………………................. 36

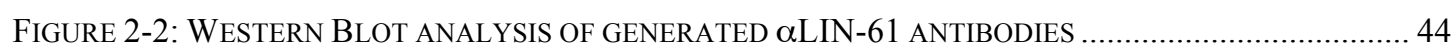

FiguRE 3-1: IDENTIFICATION OF LIN-61 AND MBTR-1 AS H3K9ME3 PEPTIDE BINDING PROTEINS ........ 57

FigURE 3-2: VERIFICATION OF LIN-61 AND MBTR-1 AS H3K9ME3 PEPTIDE BINDING PROTEINS ........... 58

FIGURE 3-3: ALIGNMENT OF LIN-61 AND MBTR-2 PROTEIN SEQUENCES WITH THE MBT REPEATS OF

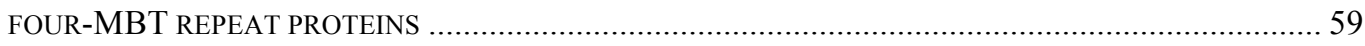

FIGURE 3-4: RECOMBINANT MBP-TAGGED LIN-61 INTERACTS SPECIFICALLY WITH H3K9ME3............... 62

FIGURE 3-5: ENDOGENOUS LIN-61 INTERACTS SPECIFICALLY WITH THE H3K9 METHYL MARK................... 63

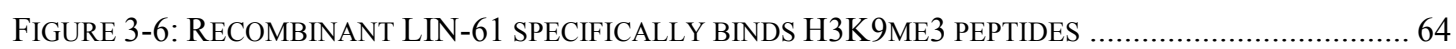

FIGURE 3-7: MBT REPEATS TWO TO FOUR OF LIN-61 ARE REQUIRED FOR H3K9ME3 PEPTIDE BINDING 66 FIGURE 3-8: D428A MUTATION RESULTS IN SLIGHT AND W455A OR F459A MUTATION IN STRONG

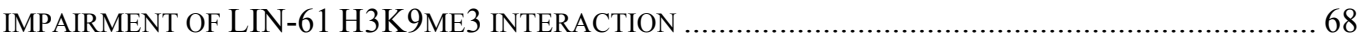

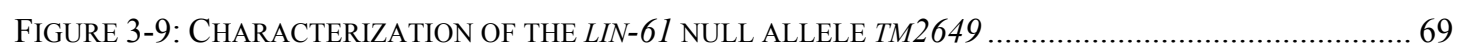

FIGURE 3-10: DESCRIPTION OF THE MBTR-1 ALLELE N4775 _................................................................. 70

FIGURE 3-11: LIN-61 AND MBTR-1 SINGLE OR LIN-61 MBTR-1 DOUBLE MUTANTS SHOW NO

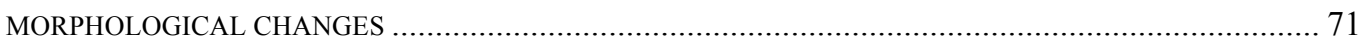

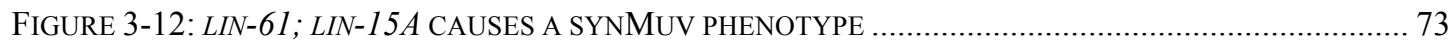

FIGURE 3-13: LIN-3 MRNA LEVEL IS ELEVATED IN LIN-61; LIN-15A …............................................ 74

FIGURE 3-14: $L I N-61 ; H P L-2\left(24.5^{\circ} \mathrm{C}\right)$ ARE MUV AND HAVE DISORGANIZED GONADAL STRUCTURES ...... 76

FIGURE 3-15: LIN-61 CAUSES REDUCED MEAN BROOD SIZE AT $24.5^{\circ} \mathrm{C}$ AND IS SYNERGISTIC TO HPL-2 AND MET-2 REGARDING STERILITY RATE AND MEAN BROOD SIZE …................................................ 79

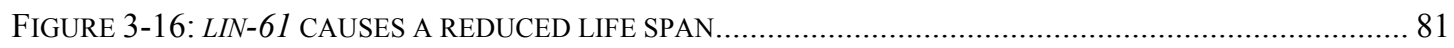

FIGURE 3-17: LIN-61 POINT MUTANTS ENCODED BY LIN-61 ALLELES WITH IMPAIRED FUNCTIONS WITHIN THE SYNMUVB PATHWAY AFFECT H3K9ME3 BINDING IN VITRO 83

FIGURE 3-18: THE MUV PHENOTYPE OF LIN-61; LIN-15A IS NOT RESCUED BY LIN-61 CONTAINING A TRIPLE AROMATIC CAGE POINT MUTATION

FIGURE 3-19: ENDOGENOUS LIN-61 EXISTS IN HIGH MOLECULAR WEIGHT COMPLEXES......................... 86

FIGURE 3-20: IDENTIFICATION OF LIN-13 AS AN INTERACTION PARTNER OF LIN-61 .......................... 89 


\section{List of tables}

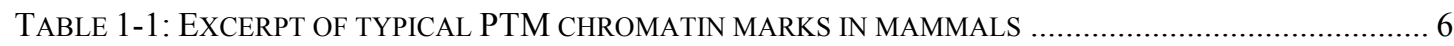

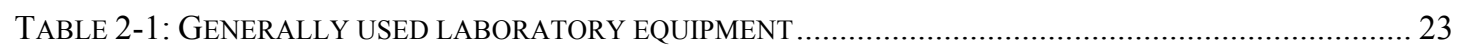

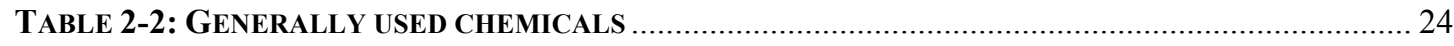

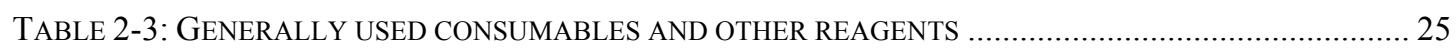

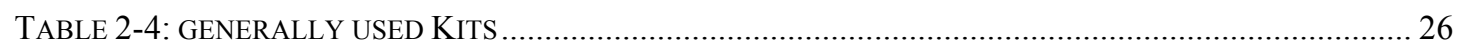

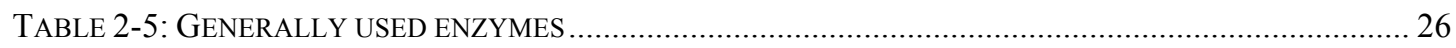

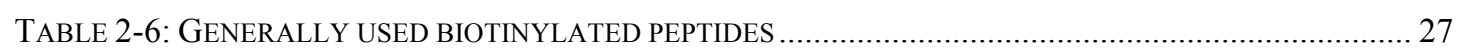

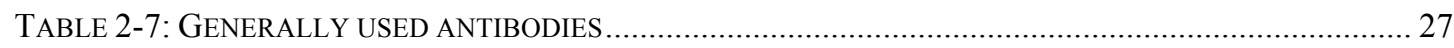

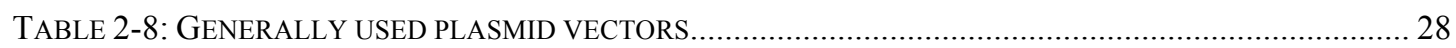

TABLE 2-9: PURCHASED PLASMIDS CONTAINING EITHER LIN-61 OR L3MBTL1 CDNA …………………..... 28

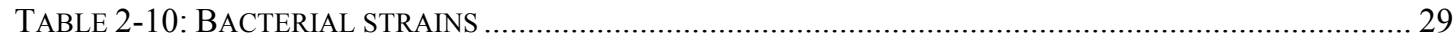

TABLE 2-11: C. ELEGANS STRAINS OBTAINED FROM DIFFERENT SOURCES .............................................. 29

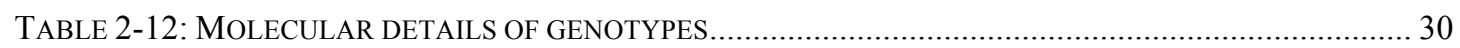

TABLE 3-1: LIN-61 CAUSES A SYNTHETIC MULTIVULVA PHENOTYPE IN COMBINATION WITH LIN-15A ..... 72

TABLE 3-2: LIN-61 CAUSES A SYNMUV PHENOTYPE BY GENETIC INTERACTION WITH MET-2 OR HPL-2 AT

$24.5^{\circ} \mathrm{C}$ 75 


\section{Abbreviations}

\begin{tabular}{|c|c|}
\hline Ac & Acetylation \\
\hline $\mathrm{AC}$ & Gonad anchor cell \\
\hline $\mathrm{ADP}$ & Adenosine diphosphate \\
\hline $\mathrm{Ar}$ & ADP-ribosylation \\
\hline ATP & Adenosine triphosphate \\
\hline BSA & Bovine serum albumin \\
\hline C. elegans & Caenorhabditis elegans \\
\hline $\mathrm{CD}$ & Chromo domain \\
\hline CENP-3 & Centromere protein 3 \\
\hline ChIP & Chromatin immunoprecipitation \\
\hline CID & Collision-induced dissociation \\
\hline DIC & Differential interference contrast microscopy \\
\hline DMSO & Dimethylsulfoxide \\
\hline DNA & Deoxyribonucleic acid \\
\hline dNTPs & Desoxyribonucleotide triphosphate \\
\hline DP & Dimerization partner \\
\hline DRM & DP, Rb class B synMuv complex \\
\hline dSFMBT & Drosophila SCM-related gene containing four MBT domains \\
\hline DTC & Distal tip cells \\
\hline DTT & DL-1,4-dithiothreitol \\
\hline E. coli & Escherichia coli \\
\hline E2F & Group of transcription factors \\
\hline EDTA & Ethylenediaminetetraacetic acid \\
\hline EGTA & Ethylene glycol tetraacetic acid \\
\hline Emb & Embryonic lethality \\
\hline FA & Fluorescence anisotropy \\
\hline FPLC & Fast protein liquid chromatography \\
\hline GFP & Green fluorescent protein \\
\hline GST & Glutathione S-transferase \\
\hline $\mathrm{H} 3 \mathrm{~K} 9 \mathrm{me} 0$ & Histone 3 lysine 9 unmodified \\
\hline H3K9me1 & Histone H3 lysine 9 monomethylation \\
\hline H3K9me2 & Histone $\mathrm{H} 3$ lysine 9 dimethylation \\
\hline H3K9me3 & Histone $\mathrm{H} 3$ lysine 9 trimethylation \\
\hline HAT & Histone acetyltransferase \\
\hline $\mathrm{HC}$ & Heavy chain of antibody \\
\hline HDAC & Histone deacetylase \\
\hline HEPES & 2-[4-(2-hydroxyethyl)-1-piperazinyl]-ethanesulfonic acid \\
\hline
\end{tabular}




\begin{tabular}{|c|c|}
\hline His & Histidine \\
\hline HMT & Histone methyltransferase \\
\hline HOX & Homeobox \\
\hline HP1 & Heterochromatin protein 1 \\
\hline HPL-2 & HP1-like-2 \\
\hline HRP & Horseradish peroxidase \\
\hline Hyp7 & Hypodermal syncytium hyp7 \\
\hline $\operatorname{Ig}$ & Immunoglobulin \\
\hline IP & Immunoprecipitation \\
\hline IPTG & Isopropylthio-b-D-galactoside \\
\hline ITC & Isothermal titration calorimetry \\
\hline L3MBTL & Human lethal (3) malignant brain tumor-like \\
\hline LC-MS/MS & Liquid Chromatography Tandem Mass Spectrometry \\
\hline LIN-61 & Protein with four MBT domains in C. elegans \\
\hline Lva & Larval arrest \\
\hline MBD & Methyl-CpG-binding domain \\
\hline MBP & Maltose binding protein \\
\hline MBT & Malignant brain tumor domain \\
\hline MBTD1 & Malignant brain tumor domain-containing protein 1 \\
\hline MBTR & MBT repeat-containing protein \\
\hline $\mathrm{Me}$ & Methylation \\
\hline \multirow[t]{2}{*}{ MET } & Methyltransferase \\
\hline & (C. elegans) \\
\hline Mi-2 & Chromatin remodeling protein $\mathrm{Mi}-2$ \\
\hline MS & Mass spectrometry \\
\hline MTA & Metastasis-associated \\
\hline Muv & Multivulva phenotype \\
\hline NGM & Nermatode growth medium \\
\hline Ni-NTA & Nickel-loaded nitrilotriacetic acid coated agarose \\
\hline NuRD & Nucleosome remodeling and deacetylase complex \\
\hline OD & Optical density \\
\hline ON & Overnight \\
\hline PBS & Phosphate buffered saline \\
\hline $\mathrm{PC}$ & Polycomb \\
\hline PCR & Polymerase chain reaction \\
\hline PEG & Polyethyleneglycol \\
\hline $\mathrm{Ph}$ & Phosphorylation \\
\hline PHD & Plant homeodomain \\
\hline PMSF & Phenylmethylsulfonylfluoride \\
\hline PRC1 & Polycomb-group repressive complex 1 \\
\hline
\end{tabular}


PRE

PTM

$\mathrm{RB}$

Rbbp

RNA

RNAi

Rpm

RT

SAM

$\mathrm{SCM}$

SCML2

SDS

SDS-PAGE

SETDB1

SUMO

SynMuv

TD

TEMED

VPC

Vul

$\mathrm{Wt}$

Zn finger
Polycomb response element

Posttranslational modifications

Retinoblastoma protein

Retinoblastoma-associated-binding-proteins

Ribonucleic acid

RNA interference

Revolutions per minute

Room temperature

Sterile-alpha-motif

Sex comb on midleg (Drosophila)

Sex comb on midleg-like 2 (Drosophila)

Sodium dodecyl sulfate

Sodium docecyl sulfate polyacrylamide gel electrophoresis

SET domain bifurcated 1

Small Ubiquitin-like Modifier

Synthetic multivulva

Tudor domain

Tetramethylethylenediamine

Vulval precursor cells

Vulvaless phenotype

Wild type

Zinc finger 


\section{Introduction}

\subsection{General view on chromatin}

In eukaryotic cells the inheritable genetic information that is composed of linear double helical DNA is stored in a cell nucleus of only a few micrometers in diameter [1]. The association of DNA with a set of histone and non-histone proteins to form a structure named chromatin effects the spatial organization of DNA by leading to compaction. The condensation of DNA is needed for packaging into the nucleus as the genome of eukaryotic cells can be very large, such as about three billion base pairs (which equates to about two meters in length) for human DNA [2]. Besides compressive function, chromatin provides an important base for the general organization of the genome.

\subsubsection{Packaging DNA into Chromatin}

In general, the structure of chromatin is based on a repeating unit of nucleosome core particles that are spaced by $\sim 10-80$ bp of linker DNA [3, 4] resulting in a 'beads on a string' configuration that leads to an approximately five-fold compaction of DNA [5]. Nucleosome core particles consist of a disc-shaped core composed of an octamer of histone proteins around which a DNA stretch of $147 \mathrm{bp}$ is wrapped in 1.65 turns [6]. The histone octamer is built by two copies of each of the four core histones H2A, $\mathrm{H} 2 \mathrm{~B}, \mathrm{H} 3$ and $\mathrm{H} 4$, which are assembled into one $\mathrm{H} 3 / \mathrm{H} 4$ tetramer and two $\mathrm{H} 2 \mathrm{~A} / \mathrm{H} 2 \mathrm{~b}$ dimers. The nucleosome, the spacing DNA and the histone H1 establish the complete chromatosome. Histones are highly conserved small basic proteins $(100-135$ aa) that share the so-called histone fold motif, a mainly $\alpha$-helical domain, in their C-terminal part for $\mathrm{H} 2 \mathrm{~B}, \mathrm{H} 3$ and $\mathrm{H} 4$ or middle part for $\mathrm{H} 2 \mathrm{~A}$. The histone proteins also share basic lysine and arginine rich N-terminal tail regions. The histone fold motifs are crucial in the organization of the nucleosome core particle by mediating histonehistone and histone-DNA interactions [7]. The N-terminal tail regions reach outside of the nucleosomal disc and have in contrast to the histone fold motifs an unstructured appearance [8].

Further compaction ( $\sim 50$-fold) of chromatin is achieved by folding into a $\sim 30 \mathrm{~nm}$ fiber of still debated structure, which is described based on two different models [3]. According to the solenoid (one-start helix) model, the nucleosomes are gradually 
coiled around a central axis, while the zigzag (two-start helix) model describes a zigzag arrangement of nuclear core particles with linker DNA connecting the nucleosomes on opposite sides of the fiber [9]. The folding of chromatin into $30 \mathrm{~nm}$ fibers is stabilized by interaction of linker histones $\mathrm{H} 1$ with nucleosomes resulting in the fixation of another approximately $20 \mathrm{bp}$ of linker DNA, while this length is dependent on chromatic area, cell type and cell cycle state $[9,10]$. The structures of further chromatin compaction are still unclear. Current models predict formation of loops of $30 \mathrm{~nm}$ fibers and additional tethering of chromatin fibers [11-13].

\subsubsection{Chromatin organization}

Although the exact structures of chromatin on higher organization levels remains to be solved, packaging of DNA into distinct chromatin states is known to play a pivotal role for the maintenance of intact chromosomes. It is an important mechanism in cellular processes like replication, recombination or mitosis as well as for regulation of gene expression. Based on characterization of interphase chromatin in 1928 by Heitz [14], the terms heterochromatin and euchromatin are used to define two major states of chromatin organization.

Heterochromatin: Within heterochromatin the packaging of chromatin is condensed throughout the cell cycle, the spacing of nucleosomes is regular, replication timing is late in S-phase, the frequency of meiotic recombination is low and the DNA is mainly inaccessible for DNA binding proteins. Heterochromatin is largely transcriptionally silent $[15,16]$. The heterochromatic state is characteristic for genomic regions that have a low gene density, but that are often rich in repetitive sequences [16].

Centromeres, pericentric regions and telomeres are packaged into heterochromatin, which fulfills in this context important functions in chromosome stability and proper mitotic chromosome segregation [17]. As the heterochromatic state within these regions is maintained permanently in all cells, it is termed constitutive heterochromatin. The term facultative heterochromatin is used to define condensed and transcriptionally silent states of chromatin that retain the potential to interconvert to a decondensed and transcriptionally active state [18]. Facultative heterochromatin are for example regions that are in a heterochromatic state in only a subset of cells (e.g. X-chromosome inactivation in mammals), on only one chromosome homolog (e.g. imprinted genomic loci) or in defined developmental stages (e.g. silencing of HOX gene clusters) [18]. 
Euchromatin: In contrast to heterochromatin, euchromatic regions are transcriptionally active, they are gene rich and their chromatin structure is defined by a less condensed but more accessible state with a rather irregular nucleosome arrangement [16]. In this more flexible chromatin state, genes can be turned on or off [19].

\subsubsection{Specification and regulation of chromatin states}

To establish, maintain, stabilize, mark and interconvert euchromatic and heterochromatic chromatin states, different mechanisms are involved.

\subsubsection{Histone variants}

Chromatin structure and function are in part regulated by the incorporation of histone variants that exist for all canonical histones but H4. Histone variants can at least in part alter the structure of nucleosomes due to a modified primary sequence [20, 21]. Also, histone variants like the H3 variant CENP-3 at centromeres designate specific chromatin states or regions. They might display specific recognition sites for effector proteins that in turn mediate downstream effects [22].

\subsubsection{Chromatin remodeling complexes}

Chromatin remodeling complexes are involved in many aspects of chromatin packaging and its dynamic regulation. All complexes have in common that they utilize the energy of ATP hydrolysis to change the state of chromatin packaging by moving, ejecting or reconstructing nucleosomes [23]. Certain chromatin remodeling complexes promote chromatin assembly, and by dense nucleosome packaging, repression of transcription. Other complexes promote the accessibility of DNA and by that facilitate many processes that need direct DNA access like transcription, replication, DNA repair or recombination [23]. As example, human NuRD (nucleosome remodeling and deacetylase) complexes are involved in gene repression. The ATPase activity in these complexes is provided by Mi-2 proteins ( $\alpha$ and $\beta$ ), which contain an ATPase domain and two chromodomains. NuRD complexes achieve diversity in regulatory functions through combinatorial assembly, and there are main accessory subunits: HDAC1 and/or HDAC2, members of the methyl CpG-binding domain (MBD) protein family, retinoblastoma-associated-binding proteins (Rbbp4 
(p48) and Rbbp7 (p46)) and members of the metastasis associated (MTA) protein family [24-26]

\subsubsection{Posttranslational histone modifications}

Posttranslational modifications (PTMs) on histones are involved in the establishment of a specific chromatin environment either directly by changes in chromatin structure or indirectly by recruitment of effector proteins. A large number of different types of PTMs on histones have been identified by mass spectrometry or by specific antibodies. Most PTMs are found on the N-terminal at histone tails, which are protruding out of the nucleosome [19]. Lysines have been identified to be acetylated, (mono-, di-, tri-) methylated, sumoylated or ubiquitylated at their $\varepsilon$-amino group. Arginines can be modified by (mono-, symmetrical di-, asymmetrical di-) methylation and serine, threonine or tyrosine can be phosphorylated [19, 27]. In all cases, enzymes have been identified that attach particular histone PTMs (writers) and others that remove the corresponding histone PTMs (erasers), indicating a dynamic process [19, 27-29]. While some enzyme classes like histone methyl transferases (HMTs), which methylate specific lysines, show a high degree of sequence specificity, others like histone acetyl transferases (HATs), show more promiscuous substrate specificity [19, 30].

\section{Distribution of histone modifications along genomic regions}

One critical function of histone PTMs is that they dictate different chromatin environments [19]. Genome wide profiling in mammalian systems using chromatin immunoprecipitation (ChIP)-based methods reveal that the spatial distribution of particular sets of histone PTMs along the genome demarcate specific chromosomal regions. Specific patterns of histone PTMs serve as signatures of specific chromosomal regions in mammals, although these are not definite, with many exceptions and dynamic changes (illustrated in Figure 1-1) [31-33]. 

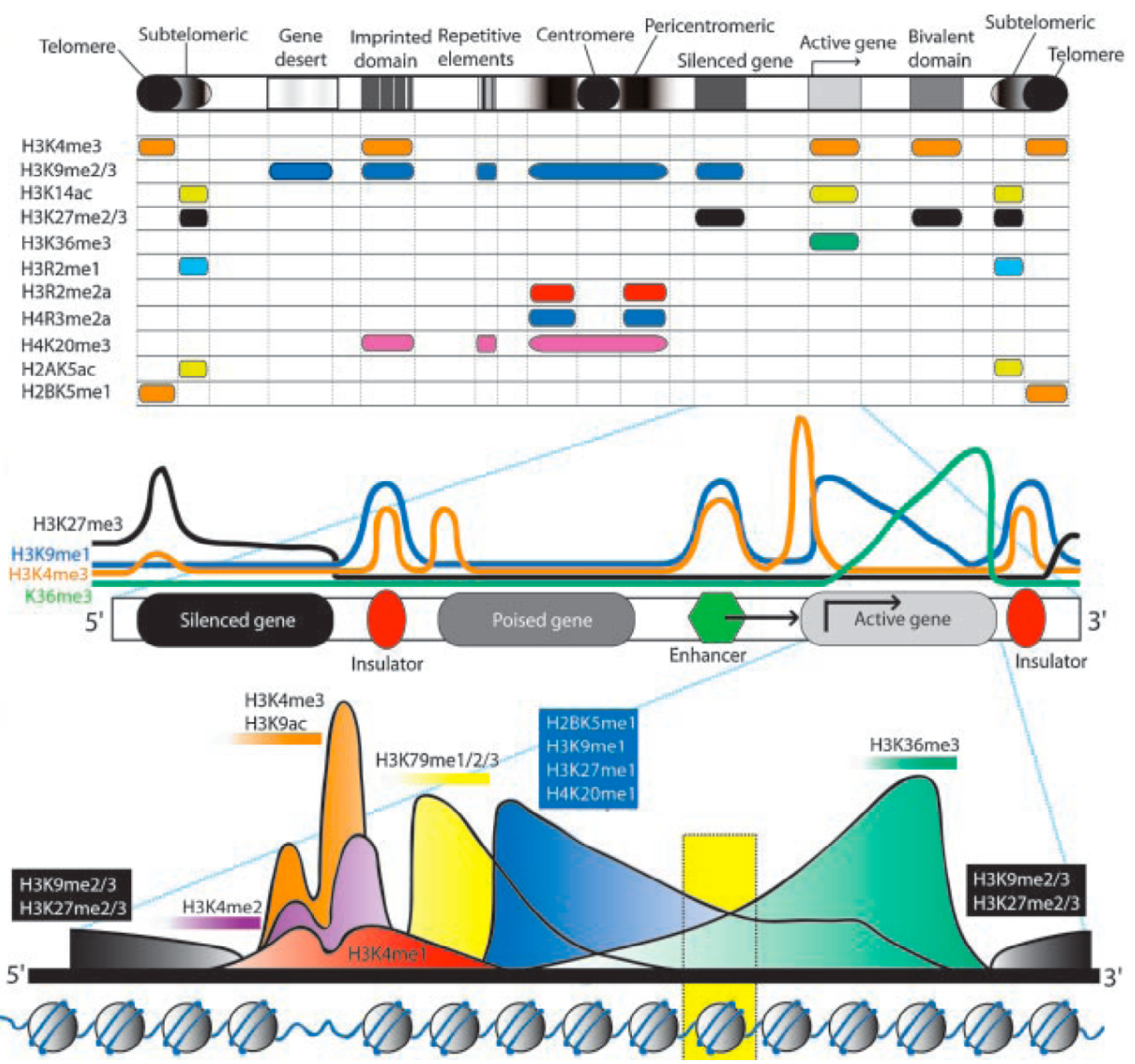

Figure 1-1: Schematic overview of histone modifications across a typical mammalian chromosome (taken from [32])

Interestingly, the three different methylation states of particular lysines (monomethyl $=\mathrm{Kme} 1, \quad$ dimethyl $=\mathrm{Kme} 2, \quad$ trimethyl $=\mathrm{Kme} 3$ ) are not necessarily distributed in similar genomic regions [32]. For example H3K9me1 could be detected together with other mono-methyl lysine marks at active gene regions in close proximity to the transcriptional start site and at insulator or enhancer regions. In contrast, $\mathrm{H} 3 \mathrm{~K} 9 \mathrm{me} 2$ and $\mathrm{H} 3 \mathrm{~K} 9 \mathrm{me} 3$ are enriched in constitutive heterochromatin regions like pericentromeric heterochromatin, repetitive elements or in non-genic regions greater than $1 \mathrm{Mb}$ ("gene-desert"). Additionally, the two marks are occurring at imprinted loci, and they are associated with gene silencing. Although $\mathrm{H} 3 \mathrm{~K} 9 \mathrm{me} 2 / \mathrm{me} 3$ is generally thought to hold repressive functions in chromatin regulation, Vakok et al. [34] could also detect $\mathrm{H} 3 \mathrm{~K} 9 \mathrm{me} 2 / \mathrm{me} 3$ in transcribed regions of active genes. 


\section{Correlation of histone modifications with biological effects}

Beside the organization of more global chromatin environments, histone PTMs are regulating the access to DNA needed for processes like transcription or DNA repair $[19,30,35]$.

As shown in Figure 1-1 and summarized in Table 1-1, numerous PTMs have been associated with either transcriptional activation or repression.

Table 1-1: Excerpt of typical PTM chromatin marks in mammals

\begin{tabular}{ll}
\hline \hline PTM chromatin marks implicated in transcriptional activation \\
\hline \hline Acetylated lysine (Kac): & $\mathrm{H} 3(9,14,18,56), \mathrm{H} 4(5,8,13,16)$, \\
& $\mathrm{H} 2 \mathrm{~A}, \mathrm{H} 2 \mathrm{~B}$ \\
Phosphorylated serine/threonine (S/Tph): & $\mathrm{H} 3(3,10,28), \mathrm{H} 2 \mathrm{~A}, \mathrm{H} 2 \mathrm{~B}$ \\
Methylated arginine (Rme): & $\mathrm{H} 3(17,23), \mathrm{H} 4(3)$ \\
Methylated lysine (Kme): & $\mathrm{H} 3(4,36,79)$ \\
Ubiquitylated lysine (Kub): & $\mathrm{H} 2 \mathrm{~B}(120)$
\end{tabular}

PTM chromatin marks implicated in transcriptional repression

\begin{tabular}{ll}
\hline \hline Methylated lysine (Kme): & H3 (9, 27), H4 (20) \\
Ubiquitylated lysine (Kub): & H2A (119) \\
Sumoylated lysine (Ksu): & H2B (6/7), H2A (126) \\
Deimination (R $\Rightarrow \mathrm{Cit})$ & $\mathrm{H} 3, \mathrm{H} 4$ \\
DNA methylation on cytosine (meC): & $\mathrm{CpG}$ islands
\end{tabular}

Adapted from $[19,27]$

Although $\mathrm{H} 3 \mathrm{~K} 36 \mathrm{me} 3$ is implicated in transcriptional activation, as this mark is enriched across actively transcribed genes, it seems to function in the suppression of inappropriate transcriptional cryptic start sites within the coding region [19, 27, 36, 37].

\section{Translating a histone mark}

Histone PTMs are thought to either modulate chromatin structure in cis by directly influencing the underlying chromatin state or in trans by recruitment of specific chromatin effector proteins, which then mediate changes in chromatin accessibility. To directly influence chromatin structure (cis acting), the particular histone PTMs must possess the capability to alter contacts between nucleosomes or the interaction 
of histones with DNA [38]. As one example H4K16ac could be identified to directly impede the formation of more condensed chromatin in vitro [39].

However, most histone PTMs in general seem to be implicated in specific recruitment of chromatin effector proteins ("readers"). These preserve specific chromatin states or mediate chromatin changes by downstream mechanisms.

\subsubsection{Chromatin effector proteins}

Chromatin effector proteins act on chromatin by interacting with histone PTMs via conserved "reader" modules that contain special binding pockets for particular PTMs (examples are given below) [40-42]. In addition to the PTMs, individual "reader" modules often recognize short peptide motifs and by that, ensure binding of a particular histone PTM in a sequence selective context and therefore sustain specific binding [41].

Acetyl-lysine marks are bound by effector proteins via a conserved binding module named bromodomain. Bromodomains specifically interact with acetyl-lysines that insert into a deep binding pocket at one end of an $\alpha$-helix bundle [42-44]. Bromodomains are found in many proteins associated with transcriptional activation and chromatin remodeling, like certain histone acetyltransferases (HATs).

Methyl-lysine marks are recognized and bound by several "reader" modules of chromatin effector proteins. The major class of methyl mark "reader" modules is the "royal family" [42, 45, 46].

"Reader" modules of the "royal family" have a $\beta$-barrel like structure (three stranded $\beta$-sheet and an $\alpha 3_{10}$-helix) in common. The chromo domain (CD), double CD, chromo barrel, tudor domain (TD), double/tandem TD and malignant brain tumor (MBT) domains belong to this protein family $[42,47]$.

All classes of methyl-lysine binding modules have in common that the methylated lysine is positioned within an aromatic cage motif, consisting of two to four aromatic residues and often one or more acidic residues. Binding takes place mainly by cation$\pi$-type interactions between the aromatic cage residues and the methylammonium moiety. Beyond that, specific individual features like hydrogen bonding and steric exclusion accomplish methylation state-specificity [42]. Dependent on the effector protein, binding of the methyl mark takes place either in a cavity insertion or in a surface groove recognition mode [42, 48, 49]. 
The two conserved Drosophila CD proteins, heterochromatin protein one (HP1) and polycomb (PC), are examples of methyl mark binding proteins that interact with a trimethyl lysine mark via their chromo domain in a surface groove recognition mode $[48,50,51]$. By specific interactions of particular residues near the methyl mark with residues in either HP1 or PC along the surface groove, binding specificity is achieved in a way that HP1 has a higher binding affinity for the H3K9me3 mark and PC for the H3K27me3 mark. By co-immunostaining experiments, HP1 is detected to co-localize with $\mathrm{H} 3 \mathrm{~K} 9 \mathrm{me} 3$ and $\mathrm{PC}$ is detected to co-localize with $\mathrm{H} 3 \mathrm{~K} 27 \mathrm{me} 2$ on polytene chromosomes [52].

Examples for proteins belonging to the "royal family" that bind methyl marks in a cavity insertion mode are all currently investigated proteins of the MBT protein family, which is described in more detail in the next section (1.2).

The mechanisms by which chromatin effector proteins exert their functions on chromatin are not fully understood, and in many cases, the concerted action of several chromatin effector proteins is thought to maintain a chromatin state or to induce changes in chromatin structure [40].

\subsection{MBT protein family}

The MBT (malignant brain tumor) protein family is a protein family that contains factors with two, three or four MBT domains stringed together in repeats. Besides MBT repeats, most MBT proteins contain other recognizable motifs, namely zinc finger motifs and/or a sterile-alpha-motif (SAM) domain [53]. Interestingly, the SAM domain is a protein-protein interaction motif [54]. In Figure 1-2, a schematic overview of MBT proteins in Drosophila, human and C. elegans is shown.

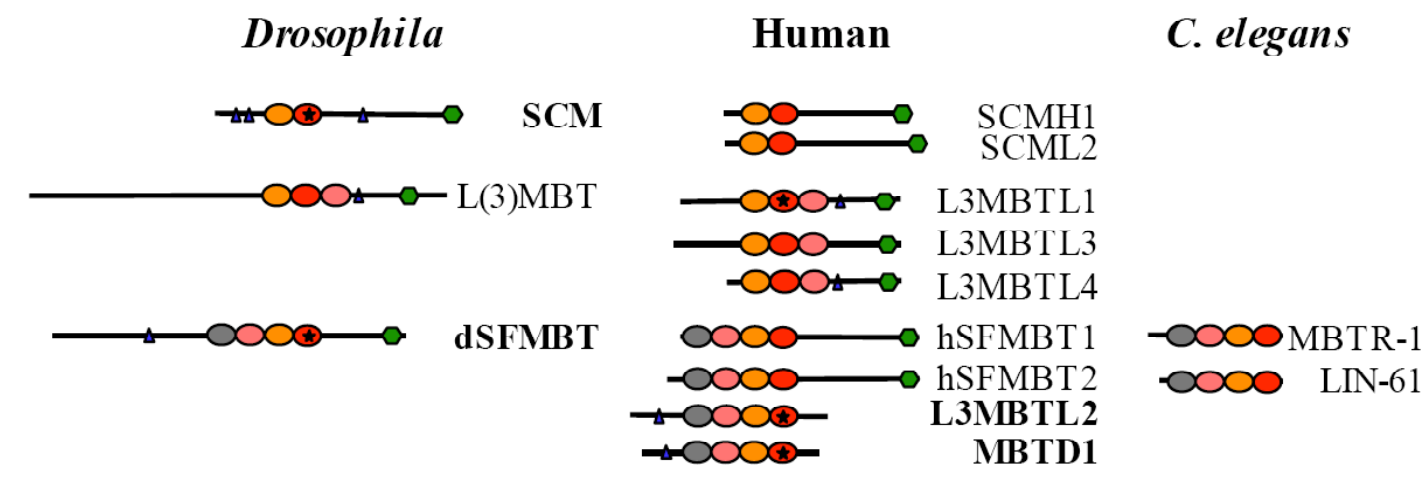

$\infty \infty_{\mathrm{MBT}}$ domains $\mathrm{SAM}$ domain $\Delta \mathrm{Zn}$ finger 
Figure 1-2: Schematic representation and domain organization of MBT proteins

Schematic overview of MBT proteins in Drosophila, human and C. elgans. The different domains are color coded according to the legend. MBT repeats that are known to interact with methyl lysine are indicated with an asterisk. Adapted from [53].

\subsubsection{Structure and function of MBT repeats}

\subsubsection{Binding of MBT repeats to methyl lysine}

The in vitro binding affinities of MBT repeats of different MBT proteins to histone tail peptides bearing either a non-, mono-, di- or tri-methylated lysine were determined by fluorescence anisotropy (FA) or isothermal titration calorimetry (ITC) measurements. Interestingly, all tested MBT repeats bind to a broad range of monoor di-methylated peptides but all show a clear discrimination against the non- or trimethylated counterparts [49, 55-59].

While other methylation mark "readers" bind their PTMs within a micromolar range, FA measurements with the MBT repeats of human L3MBTL1 for example show that the protein interacts with histone peptides mono- or di-methylated at H3K9, H3K36, H4K20 or H1K26 with affinities ranging from $5-27 \mu \mathrm{M}$ whereby the $\mathrm{K}_{\mathrm{D}}$ values for the corresponding non- or tri- methylated counterparts were at least 18 -fold higher. The binding affinities for peptides mono- or di-methylated at $\mathrm{H} 3 \mathrm{~K} 4$ or $\mathrm{H} 3 \mathrm{~K} 27$ were not as strong (ranging from 6 to $150 \mu \mathrm{M}$ ) but were nevertheless at least 5-fold stronger when compared to the non- or tri- methylated counterparts [49, 58]. ITC measurements using the MBT repeats of SCM with different peptides showed that SCM has the same binding preference to peptides regarding the lysine methylation state as other analyzed MBT proteins, but in general displays a lower affinity $\left(K_{D}\right.$ values for mono-methylated peptides $500-1200 \mu \mathrm{M}$ with no measurable interaction with non-, di- or tri-methylated peptides) [55].

In addition, it was shown that the MBT repeats of L3MBTL1 as well as of dSFMBT show strong binding towards a peptide bearing a mono-methylated lysine that is based on the $\mathrm{H}_{12-27}$ sequence but with a scrambled arrangement $[56,58]$. In contrast, no interaction of L3MBTL1 $\left(\mathrm{K}_{\mathrm{D}}>750\right)$ could be detected with H4K59me1, H4K79me1 or H3K79me1 peptides that are bearing a mono-methylated lysine within a backbone that correspond to histone core regions of $\mathrm{H} 3$ or $\mathrm{H} 4$ [58]. 


\subsubsection{Composition of methyl-lysine binding pockets within MBT proteins}

Each MBT domain is made up of an extended N-terminal arm that is basically a long loop (30 - 50 aa) and a globular core domain that is mainly formed by $\beta$-sheets building a barrel like topology (comprehending the characteristic $\beta-\beta-\beta-\alpha 3_{10}$ structural motif of the 'royal family') [47, 60, 61]. The methyl-lysine binding pocket is conserved in all analyzed methyl lysine binding MBT domains and lies within the MBT core domain. The conserved amino acids that accommodate methyl-lysine binding are arranged into a three-residue aromatic cage motif. Together with a few other hydrophobic residues, they form a hydrophobic cavity with one aromatic residue forming the bottom and the residual residues forming hydrophobic walls [59]. All analyzed co-crystal structures of MBT repeats and peptide ligands show that mono- or di-methylated lysines are perpendiculally inserted within this cavity and stabilized via van der Waals' and cation- $\pi$ interactions. In addition, the $\varepsilon$-amino group of the inserted lysine is directly hydrogen bonded and ion paired to the carboxylate of a conserved aspartate. The binding of a mono-methylated lysine is further stabilized by bridging hydrogen bonds between a proton of the lysine $\varepsilon$-amino group and a backbone carbonyl mediated by water molecules within the cavity. In contrast, the additional methyl group of a di-methylated lysine displaces the water molecule that contacts the $\varepsilon$-amino group in the case of mono-methyl lysine binding (see structural details of L3MBTL1 binding pocket in Figure 1-3 A) [49, 55-57, 59].

A

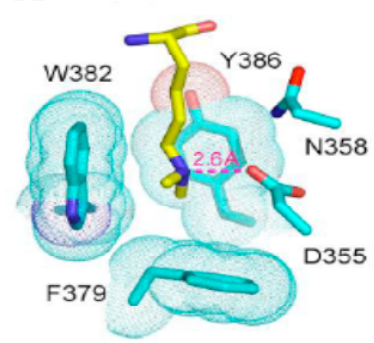

B

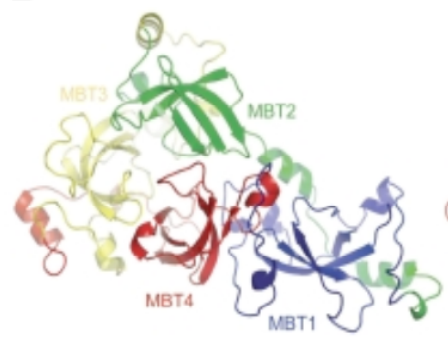

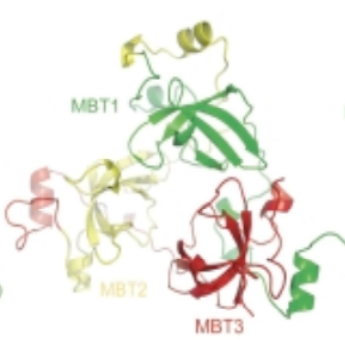

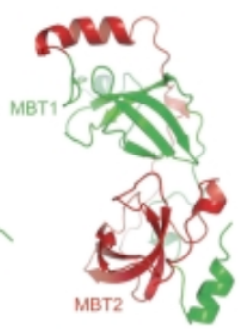

Figure 1-3: Aromatic cage motif of L3MBTL1 and ribbon diagrams of selected MBT repeats

(A) Structural details of methyl lysine binding pocket with bound di-methyl lysine of L3MBTL1. F379, W382 and Y386 build the aromatic cage. D355 is a conserved aspartate forming a hydrogen bond to the $\varepsilon$-amino group of the inserted methyl lysine (adapted from [49]) (B) Ribbon diagram of the MBT repeats of dSFMBT (left), L3MBTL1 (middle) and SCM (right). Corresponding MBT domains by comparison of their tertiary structures are depicted with equivalent colors (taken from [56]).

Selective binding of mono- or di-methylated lysines is explained by the assumption that on the one hand unmodified lysines would have much weaker van der Waals' and 
cation- $\pi$ interactions with the hydrophobic cage and that on the other hand trimethylated lysine would be too bulky to fit into the narrow binding cavity. In addition, the exchange of all ammonium protons by methyl groups results in a destruction of the hydrogen bond between the lysine $\varepsilon$-amino group and the conserved aspartate [49, 55-57, 59]. Supportive to the methyl lysine binding model are mutational studies of SCM and L3MBTL1 with ITC or FA experiments showing that the conserved aspartate within the corresponding peptide binding pocket of MBT repeat two preserves an essential function for peptide binding. In both cases an amino acid exchange to alanine causes a strongly diminished mono- or di-methyl lysine binding [49, 55, 58]. In addition, Min et al. [59] showed that each of the three conserved aromatic cage residues within MBT repeat two of L3MBTL1 is important for methyl lysine binding as the corresponding alanine exchanges result in loss of binding.

As discussed above, all tested MBT repeats recognize and bind to mono- or dimethylated lysine in a broad range of sequence contexts. However, using ITC measurements with more and more shortened H4K20me1 peptides and the MBT repeats of dSFMBT, Grimm et al. [56] showed that the peptide does contribute to some extend to the peptide-protein interaction. While a 16-residue H4K20me1 peptide is bound by the MBT repeats of dSFMBT with a $K_{D}$ value of $1 \mu \mathrm{M}$, shortening of the peptide to a three-residue peptide reduces affinity to some extent $\left(\mathrm{K}_{\mathrm{D}}=40 \mu \mathrm{M}\right)$. The fact that the broad range of mono- or di- methylated peptides that do interact with MBT repeats are all very basic (pI values 11-12) but that peptides that do not bind to MBT repeats like H3K79me1 (pI 4.4) are more acidic (see above) lead to the assumption that an overall positive charge of a peptide supports the proteinpeptide interaction $[56,58]$. This goes along with a rather negatively charged surface of dSFMBT and L3MBTL1 close to their binding pocket $[56,60,61]$. An impact of the protein surface in peptide binding could also explain why the MBT repeats of SCM show lower affinities for mono- or di- methylated peptides compared to other analyzed MBT repeats as its protein surface close to the binding pocket is less negatively charged $[56,60]$.

\subsubsection{Multi domain organization of MBT repeats}

Characteristic for MBT proteins is that they contain between two and four MBT repeats, whereby the MBT repeats are not individually stringed together but are 
arranged directly in tandem [61]. Crystal structures solved for the two MBT repeats of SCM or SCML2 show that in the case of two MBT repeats a saddle-like structure is formed whereby the extended arm of one repeat packs against the core domain of the other repeat. In addition, extensive interactions are formed between the two core domains of both MBT domains (ribbon diagram of SCM MBT repeats in Figure 1-3 B (right)) $[55,62]$. Crystallization of the three MBT repeats of L3MBTL1 showed that they adopt a triangular propeller-like architecture surrounding a central hole [61]. The propeller-like structure is established as for the two MBT repeat proteins by intensive interaction of the extended N-terminal arms with neighboring MBT core domains in a circular manner. The interdigitation of the N-terminal arm of the first repeat with the MBT core domain of the third repeat results in a conformation that puts the $\mathrm{N}$-terminus and the $\mathrm{C}$-terminus of the three-MBT repeat unit in proximity [61]. In MBT repeats of proteins with four MBT domains three MBT repeats (MBT repeat 2, 3 and 4) are composed similarly to L3MBTL1 (ribbon diagram of L3MBTL1 MBT repeats in Figure 1-3 B (middle)) while the first MBT repeat is docked onto the outer rim of the otherwise propeller-like structure [56, 57, 63]. Nonetheless the first MBT repeat sticks out of the propeller like structure, the arm of the first repeat packs against the core of the fourth repeat (ribbon diagram of dSFMBT MBT repeats Figure 1-3 B (left)) [56, 57].

Although the MBT repeats are build up at least in tandem, co-crystal structures of MBT repeats and peptide ligands indicate that in each case modified peptides are bound by only one MBT domain [55-57, 59]. Supportive to this observation are ITC measurements that show a 1:1 stoichiometry for the interaction of the MBT repeats of L3MBTL1 with mono-methylated peptides [58]. The single site of interaction is explained in MBT repeats of SCM, dSFMBT or MBTD1 by the fact that only one MBT domain is containing the conserved methyl-lysine contacting aspartate and all essential aromatic residues [55, 56, 63]. In contrast, all three MBT repeats of L3MBTL1 harbor the conserved peptide interacting residues but methyl-lysine binding of MBT repeat one or three is probably prevented by long or bulky side chains within their putative binding pockets [59]. Although only the second MBT domain of L3MBTL1 binds to methylated peptides, Li et al. [49] suggested that histone H3.3 peptides could interact with L3MBTL1 in a combinatorial readout. There H3.3P30 would insert into the binding pocket of the first MBT repeat and methylated H3.3K26/37 into the pocket of the second MBT repeat. Indeed, the 
authors showed that pocket one has the capacity to bind the dipeptide sequence proline-serine. The observation that the binding pocket of the third MBT repeat in the crystal structure of the three L3MBTL1 repeats is bound by one molecule of PEG [49] further supports the idea that MBT repeats might combine different not yet identified interaction platforms. Interestingly, based on sequence alignments neither hSFMBT1 that was shown to interact with histone $\mathrm{H} 3$ and $\mathrm{H} 4$ via its MBT repeats [64] nor hSFMBT2 contain at least one MBT repeat out of four comprising all conserved residues required for methyl-lysine binding [57].

\subsubsection{Cellular function of MBT proteins}

\subsubsection{Cellular functions of L(3)MBT and L3MBTL1}

Drosophila l(3)mbt, the first described MBT factor, was identified to be a tumor suppressor gene. A temperature sensitive l(3)mbt mutation causes malignant overgrowth of the larval brain at the restrictive temperature of $29^{\circ} \mathrm{C}$ [65]. Additionally, l(3)mbt mutations were identified to cause defects in germ-cell formation. Mutant Drosophila embryos contain a greatly reduced number of germcells. Moreover, it was shown that the synchronous mitotic divisions of the early embryo are disrupted [66]. Apart from defects derived from mutants, Koga et al. [67] showed that overexpression of human L3MBTL1 in U251MG glioma cells leads to multinucleated cells. The authors showed in addition that endogenous L3MBTL1 is associated with condensed chromatin during mitosis. They concluded that proper levels of L3MBTL1 are required to accomplish normal mitosis. Contradictory results regarding the localization of L3MBTL1 were obtained by Kalakonda et al. [58]. These authors determined the cell cycle profile of K562 cells and observed a decrease of L3MBTL1 levels in G2/M chromatin fractions but enrichment in G1/S and S phase chromatin fractions. This pattern was shown to be concordant with H4 monomethylated at lysine 20. Most recent work showed that H4K20me1 as well as Drosophila L(3)MBT are involved in a deacetylation mechanism of newly incorporated histones during chromatin assembly (a process boosted during S-phase) [68]. H4 that is found acetylated at K5 and K12 during chromatin assembly becomes deacetylated after mono-methylation of $\mathrm{H} 4$ at lysine 20 by PR-SET-7. Scharf et al. [68] argued that L(3)MBT binds to H4K20me1 and recruits HDAC activity to new 
assembled chromatin at the same time, since L(3)MBT and Rpd3 (a histone deacetylase) were shown to interact with each other.

Besides the suggested contribution of L(3)MBT in chromatin maturation, several studies indicate that both Drosophila L(3)MBT and human L3MBTL1 are involved in gene repression. It was shown that L3MBTL1 and even the three MBT repeats only are capable to repress luciferase transcription when tethered to the GAL4 binding sites of an transcriptional reporter either by binding to PR-SET-7 (GAL4-tagged) mediated H4K20me1 modification or directly by using GAL4-tagged L3MBTL1 [58, 69]. In addition, ChIP experiments revealed that L(3)MBT as well as L3MBTL1 occupy promoters of defined E2F target genes and thereby promote their repression $[58,70,71]$. Repression might be indirect via recruitment of repressor complexes (e.g. HP1 $\gamma$ and RB have been co-purified with L3MBTL1). However, there is also evidence for L3MBTL1 mediated direct compaction of nucleosomal arrays bearing either H4K20me1 or H1K26me1 marks [71].

\subsubsection{Cellular functions of SCM and dSFMBT}

The two MBT factors, SCM and dSFMBT are essential in Drosophila development as embryos lacking either SCM or dSFMBT die at the end of embryogenesis or in the larval stage $[72,73]$. In both cases, the animals show widespread misexpression of HOX genes, which assigns scm and $\mathrm{d} s f m b t$ to the Polycomb group (PcG) genes. In line with this finding, SCM was identified to be part of the PRC1 complex (a complex involved in HOX gene silencing) [74] although in substoichiometric amounts [75]. SFMBT interacts with the PcG protein $\mathrm{dPHO}$, forming the PHO-RC complex [73]. ChIP experiments revealed that SCM and dSFMBT both occupy cis-regulatory polycomb response elements (PREs) of HOX genes [56].

In functional assays, the expression of HOX genes was analyzed in clones of imaginal disc cells homozygous for either a dsfmbt or a scm knockout allele. In both cases misexpression of normally silenced $\operatorname{HOX}$ genes was observed [55, 56, 73]. Interestingly imaginal disc cells with mutations in both genes show synergistic effects on the misexpression of HOX genes and a tumor-like phenotype [56]. The presumably repressive function of SCM and dSFMBT is strengthened by the observation that artificial tethering of dSFMBT to a reporter gene causes transcriptional repression of the reporter [73]. The observation that a $\mathrm{SCM}^{\triangle \mathrm{MBT}}$ or a SCM point mutant protein in which the conserved aspartate of the binding pocket in 
MBT repeat two is replaced by an alanine were not able to repress the HOX gene $U b x$ as efficiently as a wild-type SCM protein leads to the assumption that functional MBT repeats are important for gene repression [55].

As dSFMBT was identified to exert a repressive function on reporter genes in two different genome-wide RNAi screens in which the recruitment to the reporter took place by different mechanisms, it is likely that dSFMBT is involved in gene repression apart from PHO mediated HOX gene silencing [70, 76]. Lu et al. [70] showed that a reporter gene with E2F transcription factor binding sites at its promoter is derepressed upon dSFMBT knockdown and Stielow et al. [76] demonstrated that dSFMBT mediates SUMOylation-dependent transcriptional repression.

Also, it was shown that dSFMBT, apart from HOX gene silencing, seem to play a role in germ cell development as germ cells that lack dsfmbt fail to develop [73].

\subsection{Chromatin regulation in $C$. elegans}

Caenorhabditis elegans (C. elegans) is a powerful model organism to study chromatin regulation in cellular and developmental processes as it is a very simple organism (only around 1000 somatic cells and a similar amount of germ cells). $C$. elegans has a similar complement of chromatin factors to that of humans and probably similar sets of histone PTMs as the C. elegans histones H3 (CeHIS3) and H4 (CeHIS4) are $97 \%$ and $98 \%$ identical to the human counterpart $[77,78]$.

Supportive to the assumption that chromatin regulation takes place by similar mechanisms as in other organisms is the finding that the $\mathrm{H} 3$ tail modifications $\mathrm{H} 3 \mathrm{~K} 4 \mathrm{me} 3$, H3K36me3 and $\mathrm{H} 3 \mathrm{~K} 9 \mathrm{me} 3$ are similarly distributed in C. elegans genes as in other organisms with $\mathrm{H} 3 \mathrm{~K} 4 \mathrm{me} 3$ near transcriptional start sites, H3K36me3 within the gene body and $\mathrm{H} 3 \mathrm{~K} 9 \mathrm{me} 3$ enriched on silent genes [78].

Indeed several histone PTMs and homologs of proteins known to be involved in chromatin regulation in other organisms hold functions in cellular and developmental processes in C. elegans like correct vulval development (see below 1.3.1), germline development, repetitive transgene silencing or somatic gonad development (for reviews see $[77,79])$. 


\subsection{1 synMuvB pathway in C. elegans}

\subsubsection{Vulva development in C. elegans}

The initial point for vulval development in C. elegans is in the L1/L2 larval stage during postembryonic development when six vulval precursor cells (VPCs) that are designated as P3.p, P4.p, P5.p, P6.p, P7.p and P8.p are generated from progenitor cells along the ventral mid-line $[80,81]$. During the larval L3 stage, three of those cells (P4.p, P5.p, P6.p) that are located in close proximity to the somatic gonad anchor cell (AC) become specified to generate vulval cells by the concerted interaction of several signaling cascades that are induced by the activation of a RAS/MPK-cascade [LET-60 (RAS) - LIN-46 (RAF) - MEK-2 (MEK) - SUR-1 (MAPK)]. The RAS/MPK-cascade itself is induced within these cells by binding of the epidermal growth factor ligand LIN-3 that is secreted by the AC cell. This event activates LET23, an epidermal growth factor tyrosine kinase receptor that is exposed on each VPC. Dependent on the distance to the AC cell and on signaling between the VPCs, the VPCs develop to vulval lineages of the $1^{\circ}$ (P5.p and P7.p) or $2^{\circ}$ (P6.p) type and will generate distinct sets of progeny to constitute the vulva. The residual three VPCs (P3.p, P4.p, P8.p) generate a $3^{\circ}$ lineage, which makes epidermal cells that fuse with the large syncytial epidermis hyp7 [80-83]. For correct vulval development, it is crucial that the dose of LIN-3 that can interact with exposed LET-23 on all six VPCs is fine-tuned. The VPC P6.p receives most, the neighboring two VPCs (P5.p, P7.p) some but the residual VPCs no LIN-3 signal [81] (Figure 1-4). While hermaphrodites with lin-3 mutations show a vulvaless (Vul) phenotype [84, 85], transgenic animals expressing elevated levels of lin-3 show a multivulva (Muv) phenotype [86]. Likewise, a gain of function mutation in let-60 causes a Muv phenotype [87]. Worms with a Muv phenotype typically have one functional vulva besides additional ventral protrusions formed from vulval tissue (pseudovulvae) that are caused by the fact that more than three VPCs adopt vulval fates.

\subsubsection{Negative regulation by synMuv genes}

Besides mutations that directly influence the signaling cascades within the VPCs, Muv phenotypes are also caused by loss of function mutations in so called synthetic multivulva (synMuv) genes. SynMuv genes can be classified into two groups (synMuvA and synMuvB), based on the observation that single or multiple loss of 
function mutations in genes of only one class do not cause a robust Muv phenotype whereas the combination of a loss of function mutation in each of the two classes cause a strong Muv phenotype [80, 88]. The two classes were assigned by Ferguson $\&$ Horvitz [88] categorizing a mutation that causes a robust Muv phenotype in a lin$8(n 111)$ background into the synMuvB class and a mutation that causes a robust Muv phenotype in a lin-9(n112) background into the synMuvA class.

Epistasis analysis showed that synMuv genes act upstream of let-23 and also upstream of lin-3 [81, 82, 89, 90]. Despite of the obvious assumption that synMuv genes might act within either the AC cell or the VPCs to repress ectopic vulval cell fate development, genetic mosaic analysis as well as tissue specific expression of the synMuvB gene lin-35 support the hypothesis that they instead act in the hypodermal syncytium hyp7 [91, 92]. Both above indicated observations are accomplished in the study of Cui et al. [89] as they could show that loss of lin-3 activity in hyp7 by tissue specific expression of a lin-3 hairpin-forming construct causes a suppression of Muv phenotype in synMuvA; synMuvB double mutants. Furthermore lin-3 mRNA levels are elevated in synMuvA; synMuvB double mutants. These results lead to the hypothesis that synMuv genes are involved either directly or indirectly in the repression of lin-3 in hyp7 and that ectopic expression of lin-3 is the main cause of the synMuv phenotype [89] (see model in Figure 1-4).

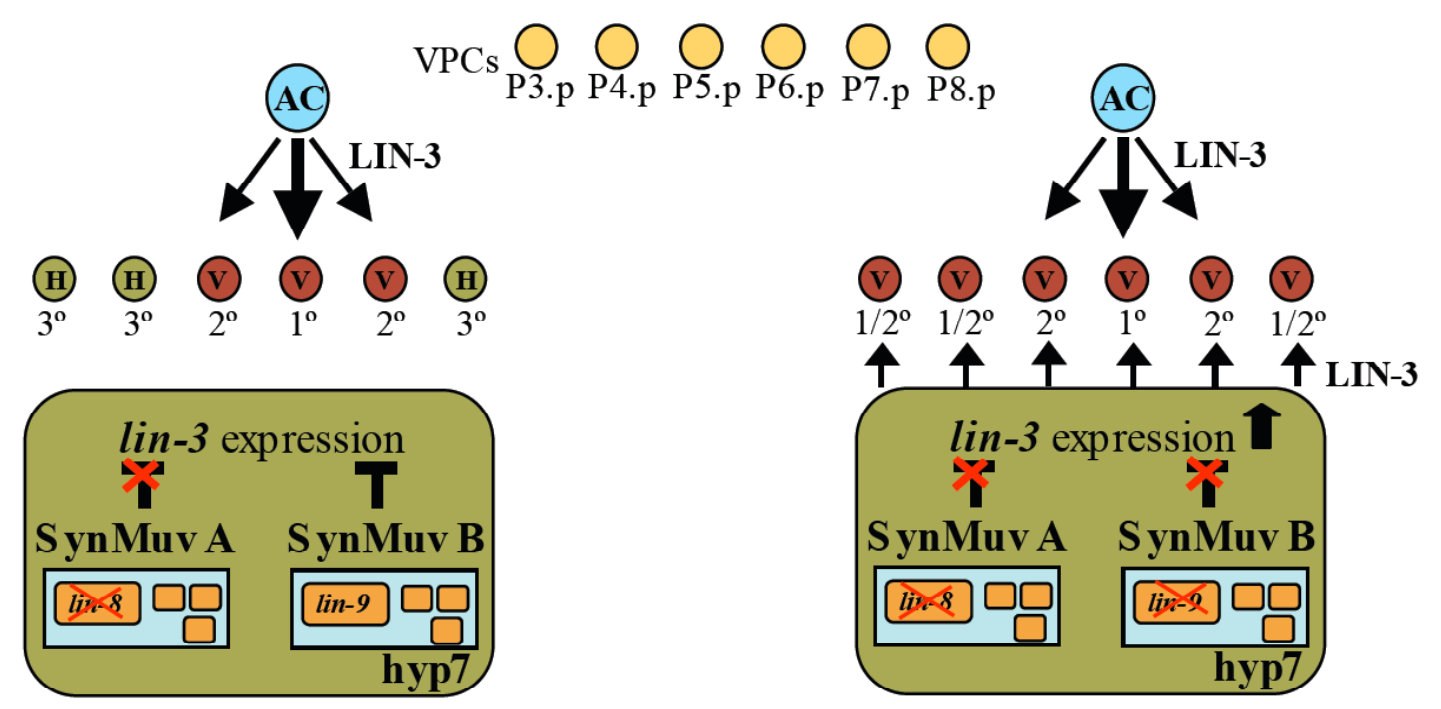

Figure 1-4: Model for the molecular basis of how synMuvA and synMuvB genes regulate ectopic vulval induction

In wild-type or by loss of function of either a synMuvA or a synMuvB gene only three (P5.p, P6.p, P7.p) of 6 vulval precursor cells (VPCs) are induced to adopt into vulval cell fate (V) by receiving a 
LIN-3 signal from the nearby anchor cell (AC). The other three VPCs (P3.p, P4.p, P8.p) receive no LIN-3 signal and divide once to produce daughters that fuse to hyp7 $(\mathrm{H})$ as lin-3 expression is inhibited in the surrounding hyp7 syncytium redundantly by synMuvA and synMuvB genes. In synMuvA; synMuvB double mutant worms (like lin-61; lin-15A), lin-3 expression in hyp7 is derepressed and elevated levels of LIN-3 induces all 6 VPCs to adopt into vulval cell fates. Adapted from [77, 89].

Interestingly the mechanisms by which synMuvA and synMuvB genes act on repression seem to be temperature sensitive. SynMuvA; synMuvB pairs show a higher prevalence of the Muv phenotype at higher temperatures [80, 88]. Despite detailed genetic studies, the exact molecular mechanisms of SynMuv factors have not been worked out so far.

\subsubsection{The synMuvB pathway}

While most synMuvA genes (lin-8, lin-15A, lin-38, lin-56) encode proteins of unknown function, many of the synMuvB genes encode proteins implicated in chromatin remodeling and transcriptional repression [80]. A number of synMuvB proteins have been identified to be associated in distinct complexes.

DRM complex: LIN-35, the only $C$. elegans homolog of the mammalian retinoblastoma tumor suppressor protein $\mathrm{RB}$ could be identified as a synMuvB factor. Further EFL-1 and DPL-1 are homologues to either the E2F transcription factor family or the dimerization-partner (DP) family, respectively [93, 94]. The EFL-1 protein is most similar to the Drosophila dE2F2 as well as to the mammalian E2F-4 protein, both belonging to a set of E2F transcription factors that are implicated in repression, known to form hetero-dimers with DP proteins and known to associate with RB proteins [93, 95, 96]. Indeed Ceol \& Horvitz [93] could show by in vitro GST pull-down experiments that EFL-1, DPL-1 and LIN-35 do interact with each other. In 2004 two intriguing studies showed that in Drosophila, the two RB proteins RBF1 and RBF2 not only interact with dE2F2 and DP but form bigger complexes that additionally contain the dMyb protein as well as Mip130, Mip120, Mip20 and CAF1/p55 [93, 97, 98]. Moreover, Lewis et al. [98] could identify dLIN-52 to be part of this core complex. The MBT protein L(3)MBT as well as the HDAC Rpd3 were found to be associated substoichiometrically. The complex identified by Korenjak et al. [97] is named dREAM while Lewis et al. [98] named the complex Myb-MuvB as all identified proteins but Myb have homologs in C. elegans that belong to the group of synMuvB proteins. Both studies suggest that the identified complexes are involved in gene repression as they showed that depletion of selected complex members lead to 
de-repression of E-group genes. In addition, Korenjak et al. [97] detected no overlapping staining pattern of selected complex members with the pattern of phophorylated RNA polymerase II on polytene chromosomes indicating a primarily association with transcriptional silent regions.

In 2006, Harrison et al. [99] showed that all C. elegans homologues proteins of the Drosophila core Myb-MuvB complex are associated in a complex in nuclear extract, which they named DRM complex. The DRM complex is composed of the synMuvB proteins RB (RBF1/RBF2), EFL-1 (dE2F2), DPL-1 (dDP), LIN-9 (Mip130), LIN-54 (Mip120), LIN-37 (MIP-40), LIN-53 (Caf1/p55) and LIN-52 (dLIN-52) but neither HDA-1 (Rpd3) nor the MBT protein LIN-61 could be co-precipitated with $\alpha$ LIN-37 or $\alpha$ LIN-9 antibodies [99]. In support, no proteins of the DRM complex could be coprecipitated with $\alpha \mathrm{LIN}-61$ antibodies [100].

NURD complex: Besides the DRM complex, conserved components of the NuRD complex belong to the synMuvB class. However these are not identified to form complexes in C. elegans. Namely: LET-418 (Mi-2), HDA-1 (HDAC1/2; Rpd3) and LIN-53 (RbAP48; Cafl/p55) [94, 101, 102]. It is worth mentioning that LIN-53 is also part of the DRM complex (see above). In addition, MEP-1 is a synMuvB factor [103]. Interestingly it was shown in Drosophila that dMi-2 and dMEP-1 form a complex involved in transcriptional repression separate from NuRD [104].

TIP60/NuA4-like histone actetyltransferase complex: In 2004, Ceol \& Horvitz [105] identified loss of function of mys-1, trr-1 or epc-1 to produce a synMuv phenotype in a synMuvA mutant background. As MYS-1 is a homologue of the mammalian Myst histone acetyltransferase TIP60 (Esa1 in yeast) and because EPC-1 and TRR-1 are homologues to yeast proteins, which are identified to be in a complex with Esal [106], Ceol \& Horvitz propose that a TIP60 complex might act in vulval cell fate specification. TIP60 complexes are implicated to function in DNA repair [107-109] but also in both transcriptional activation and repression [110, 111].

MET-1 and MET-2 are the only two HMTs out of 38 predicted lysine-specific HMTs in $C$. elegans shown to cause a Muv phenotype in a synMuvA mutant background [101, 112]. As H3K36me3 methylation is strongly decreased and H3K9me3 methylation is slightly decreased in met-1 mutant worms, met-1 (similar to yeast Set2) is assumed to encode a H3K36 HMT. Met-2 (similar to human SETDB1) in 
contrast is assumed to encode a H3K9 HMT as met-2 mutant worms show the opposite methylation pattern compared to met-1 mutant worms [112]. In rescue experiments, Andersen et al. [112] could demonstrate that mutations that abolish the enzymatic activity of either MET-1 or MET-2 impede their ability to rescue the synMuv phenotype of met-1; lin-15A or met-2; lin-15A double mutant worms, indicating that the enzymatic activity of met-1 and met-2 plays important roles within the synMuvB pathway.

HPL-2, one out of two HP-1 homologues in C. elegans, was shown to act in the synMuvB pathway [113-115]. Interestingly, it interacts with another synMuvBcategorized factor, the multi zinc finger protein LIN-13 [116].

In contrast to $h p l-2$, a mutation of the other C. elegans HP-1 homolog named hpl-1 does not cause a synMuv phenotype in a loss off function background of several synMuvA or synMuvB genes [113-115]. Nevertheless $h p l-2$; $h p l-1$ double mutant worms exhibit a strong Muv phenotype [114].

As HPL-2 is present in the same pathway as the H3K9me HMT MET-2, it was suggested that HPL-2 exert its repressive function within the synMuvB pathway by interaction with the H3K9me3 mark. However, Coustham et al. [116] found that HPL-2::GFP does not significantly overlap with the H3K9me3 mark but that its localization is dependent on the presence of LIN-13. In contrast, Wirth et al. [117] showed that endogenous HPL-2 does interact with H3K9me3 peptides in a peptide pull-down assay.

LIN-61 is another protein identified to act as a synMuvB factor $[100,101]$ and it belongs to the MBT protein family. Therefore it is likely that LIN-61 act as a chromatin effector protein within the synMuv pathway. Newsworthy is that the second MBT factor of C. elegans, MBTR-1, is not involved in vulval development [100].

Interestingly, from 18 genes that Poulin et al. [101] identified in a genome wide RNAi screen to act as synMuv genes, three belong to the sumoylation pathway. Apart from sumo (SMO-1) itself, the sumo activating enzyme UBA-2 as well as the E2 sumo conjugating enzyme UBC-9 seem to function in the repression of ectopic vulval development. Of interest is that none of the three genes can be classified into the synMuvB category. Only the depletion of any of the three factors in lin- $15 B$ (synMuvB gene) as well as in lin-15A (synMuvA gene) mutant background result in prevalent muv phenotypes [101]. 
It has to be mentioned that some synMuv genes like lin-13 exhibit a Muv phenotype to some extent without any further loss-of-function mutation in either class A or B synMuv genes. A lin-13 mutation causes a Muv phenotype at $25^{\circ} \mathrm{C}$ but is synthetic to synMuv A genes at $15^{\circ} \mathrm{C}[84,118]$.

Although the above described genes are categorized into the two different classes synMuvA or synMuvB, certain synMuv genes like the sumoylation genes (see above) defy clear-cut categorization [80, 101]. Another example is hpl-2. hpl-2 loss-offunction causes a penetrant Muv phenotype in all tested synMuvA mutant backgrounds but additionally in a subset of synMuvB mutant backgrounds (lin-15B, lin-9, lin-35, lin-37, met-1, met-2) however not in a lin-36 or lin-53 (both synMuvB) mutant background $[112-114,116]$. These observations lead to the assumption that some genes of the synMuvB class might act redundantly in the repression of ectopic vulval cell-fate development [119]. Encouraged by this hypothesis, Andersen et al. [119] analyzed Muv rates and lin-3 expression levels for several combinations of synMuvA gene mutations with double synMuvB gene mutations and vice versa. Their results show that nearly all tested synMuvB double mutations in a synMuvA mutant background cause a more penetrant Muv phenotype and a more elevated lin-3 expression level as compared to the corresponding single synMuvB mutations in the same synMuvA mutant background. Based on these results, Andersen et al. [119] concluded that most synMuvB genes are redundant with each other. The authors suggested that most synMuvB genes act in separate pathways within the superordinate synMuvB pathway.

\subsubsection{Biological function of synMuvB factors apart from vulval development}

Beside their involvement in negative regulation of ectopic vulval-cell fates, several synMuvB genes have broader functions in C. elegans. While some synMuvB gene mutations cause no obvious effects apart from the described synMuv phenotype (e.g. lin-35; [94]), other synMuvB gene mutations cause more severe phenotypes. For example loss-of-function of lin-53, efl-1 or epc-1 cause embryonic lethality (emb) $[93,94,105]$ whereas loss-of-function of let- 418 (at $20^{\circ} \mathrm{C}$ ) or $l i n-13$ (at $25^{\circ} \mathrm{C}$ ) cause larval arrest (lva) when maternal and zygotic activities are removed and sterility when animals are segregating from let-418/+ or lin-13/+ hermaphrodites [102, 118]. hpl-2 mutation causes $44 \%$ sterility at $24^{\circ} \mathrm{C}$ and up to $100 \%$ sterility at $25^{\circ} \mathrm{C}$ but wild-type appearance at $20^{\circ} \mathrm{C}[114]$. 
Besides the suggested repressive function of synMuvB factors on lin-3 expression, several synMuvB factors seem to be implicated in gene repression of a broader spectrum of genes. A lag-2::gfp reporter is ectopically expressed in the intestine in many mutant synMuvB gene backgrounds (met-1, met-2, hpl-2, hda-1, let-418, mep2, lin-53, lin-35, lin-9, lin-54, smo-1, ubc-9, uba-2, lin-15B). Although strong expression of lag-2::gfp is restricted to the two distal tip cells and the vulva in wildtype background $[101,112,120]$.

Loss-of-function of many synMuvB genes (lin-61, hpl-2, met-1, met-2, lin-35, lin-53, $d p l-1$, efl-1, lin-15B) are detected to suppress vulval defects (distinct from Muv) caused by the partial loss-of-function allele of the gene mat-3, presumably by the ectopic activation of mat-3 expression [100, 112, 121]. In addition, several synMuvB genes seem to be implicated in the repression of at least some germline restricted genes in somatic cells. Wang et al. [122] showed that mutations in lin-35, dpl-1, lin-9, lin-13, hpl-2 or lin-15B cause ectopic expression of $p g l-1$ (encodes a component of $\mathrm{P}$ granules) apart from the germline.

\subsection{Objective of the presented thesis}

Histone PTMs in C. elegans are key elements for chromatin state maintenance and regulation. Several homologues of proteins implicated in chromatin regulation in other organisms have been found to be involved in developmental and cellular processes in C. elegans $(1.3,[77])$. C. elegans is a simple, multicellular organism and therefore powerful to investigate chromatin regulation in cellular and developmental processes. Many putative chromatin effector proteins were analyzed by phenotypic characterization of C. elegans loss-of-function mutants or RNAi treated worms. But apart from a study published while the present study was ongoing [117] in which the interaction of endogenous HIS-24 and HPL-2 to modified histone tail peptides is shown, direct interaction of putative chromatin effector proteins with histone PTMs was not investigated in C. elegans before.

In the present study it is attempted to identify and to investigate identified chromatin factors directly involved in the readout of the repressive chromatin mark H3K9me3 in C. elegans. 


\section{Materials and methods}

\subsection{Materials}

\subsubsection{Laboratory equipment}

Generally used laboratory equipment is listed in Table 2-1

Table 2-1: Generally used laboratory equipment

\begin{tabular}{|ll|}
\hline \hline Equipment & Supplier \\
\hline ÄKTA Explorer FPLC System & GE Healthcare, Buckinhamshire (UK) \\
\hline Balances & Mettler-Toledo, Giessen \\
\hline Bioruptor & Diogenode, Liege (Belgien) \\
\hline Branson Digital Sonifier & $\begin{array}{l}\text { Heinemann Ultraschall- und Labortechnik, } \\
\text { Schwaebisch Gmuend }\end{array}$ \\
\hline Centrifuge Sorwall Evolution RC & Thermo Fisher Scientific, Rockford (USA) \\
\hline Centrifuges 5415R / 5810R & Eppendorf, Hamburg \\
\hline EmulsiFlex-C5 High Pressure Homogenizer & Avestin, Ottawa (K) \\
\hline PCR machine Eppendorf Mastercycler epgradientS & Eppendorf, Hamburg \\
\hline Freezer -20 ${ }^{\circ}$ C & Liebherr, Bulle (CH) \\
\hline Freezer -80 ${ }^{\circ}$ C & Thermo Fisher Scientific, Rockford (USA) \\
\hline Hera Safe sterile hood & Heraeus, Hanau \\
\hline Hereaus Kelviton Incubator & Thermo Fisher Scientific, Rockford (USA) \\
\hline Kodak X OMAT 2000 processor & Carestream Health, New York (USA) \\
\hline Mini-PROTEAN electrophoresis system & BIO-RAD, Muenchen \\
\hline Mini-Trans-Blot system & BIO-RAD, Muenchen \\
\hline Multitron shaker & HT Infors, Braunschweig \\
\hline NanoDrop ${ }^{\circledR}$ ND-1000 & Peqlab, Erlangen \\
\hline Inverted Research Microscope, ECLIPSE Ti-E & Nikon, Kingston (UK) \\
\hline NuPAGE ${ }^{\circledR}$ Pre-cast system & Incitrogen, Karlsruhe \\
\hline Olympus SZX10 & Olympus, Hamburg \\
\hline pH meter & Mettler-Toledo, Giessen \\
\hline Power Supply PowerPac Universal & BIO-RAD, Muenchen \\
\hline Rotilabo ${ }^{\circledR}-$ microhomogenisator & Roth, Karlsruhe \\
\hline Scanner Perfection V750 Pro & Epson, Meerbusch \\
\hline Sub-Cell-GT Agarose gel electrophoresis System & BIO-RAD, Muenchen \\
\hline Thermomixer comfort & Eppendorf, Hamburg \\
\hline UV Transilluminator & BIO-RAD, Muenchen \\
\hline Vortex Genie 2 & Scientific Industries \\
\hline Waterbath TW12 & Julabo, Seelbach \\
\hline Real-time PCR machine DNA Engine OPTICON & Bio-Rad, Muenchen \\
\hline Micromanipulator & Eppendorf, Hamburg \\
\hline & \\
\hline
\end{tabular}




\subsubsection{Chemicals}

Chemicals used for buffers and media are listed in Table 2-2

Table 2-2: Generally used chemicals

\begin{tabular}{|c|c|}
\hline Chemical & Manufacturer \\
\hline $100 \mathrm{mM}$ dNTP Set & Invitrogen, Karlsruhe \\
\hline 2-Mercaptoethanol & Sigma-Aldrich, Steinheim \\
\hline $\begin{array}{l}\text { 4-(2-Hydroxyethyl)-1-piperazineethanesulfonicacid } \\
\text { (HEPES) }\end{array}$ & VWR, Poole \\
\hline 5-fluorodeoxyuridine (FUDR) & Sigma-Aldrich, Steinheim \\
\hline Acetic acid & Merck, Mannheim \\
\hline Acrylamide:bisacrylamide $(37.5: 1)$ & Merck, Mannheim \\
\hline Agar & Roth, Karlsruhe \\
\hline Agarose & Serva, Heidelberg \\
\hline Ammonium chloride $\left(\mathrm{NH}_{4} \mathrm{Cl}\right)$ & Merck, Mannheim \\
\hline Ammonium persulfate (APS) & AppliChem GmbH, Darmstadt \\
\hline Ampicillin & AppliChem GmbH, Darmstadt \\
\hline Benzamidine & Sigma-Aldrich, Steinheim \\
\hline Boric acid $\left(\mathrm{H}_{3} \mathrm{BO}_{3}\right)$ & Merck, Mannheim \\
\hline Albumin, from bovine serum (BSA) & Sigma-Aldrich, Steinheim \\
\hline Bromphenol blue & Serva, Heidelberg \\
\hline Calcium chloride $\left(\mathrm{CaCl}_{2}\right)$ & Roth, Karlsruhe \\
\hline Chloroform & Merck, Mannheim \\
\hline Cholesterol & Sigma-Aldrich, Steinheim \\
\hline Citric acid monohydrate & Alfar Aesar, Karlsruhe \\
\hline Cobalt chloride $\left(\mathrm{CoCl}_{2}\right)$ & Riedel-de Haen,Seelze \\
\hline Coomassie briliant blue & BIO-RAD, Muenchen \\
\hline Copper chloride $\left(\mathrm{CuCl}_{2}\right)$ & Merck, Mannheim \\
\hline Copper sulfate $\left(\mathrm{CuSO}_{4}\right)$ & Merck, Mannheim \\
\hline Dimethyl pimelimidate $\cdot 2 \mathrm{HCl}(\mathrm{DMP})$ & Pierce, Rockford (USA) \\
\hline Dimethylsulfoxide (DMSO) & Sigma-Aldrich, Steinheim \\
\hline Dipotassium hydrogen phosphate $\left(\mathrm{K}_{2} \mathrm{HPO}_{4}\right)$ & Roth, Karlsruhe \\
\hline Dithiothreitol (DTT) & Alexis Biochemicals, Loerrach \\
\hline Ethanol & Merck, Mannheim \\
\hline Ethanolamine & Sigma-Aldrich, Steinheim \\
\hline Ethidium bromide & Roth, Karlsruhe \\
\hline Ethylenediaminetetraacetate (EDTA) & Roth, Karlsruhe \\
\hline Gelatin from bovine & Sigma-Aldrich, Steinheim \\
\hline Glucose & Merck, Mannheim \\
\hline Glutathione reduced & Sigma-Aldrich, Steinheim \\
\hline Glycerol & Merk, Mannheim \\
\hline Glycine & Merck, Mannheim \\
\hline Hydrochlorid acid $37 \%(\mathrm{HCl})$ & Merck, Mannheim \\
\hline Imidazole & Roth, Karlsruhe \\
\hline Iron chloride $\left(\mathrm{FeCl}_{3}\right)$ & Roth, Karlsruhe \\
\hline Isoamyl alcohol & Sigma-Aldrich, Steinheim \\
\hline Isopropyl beta-D-thiogalactopyranoside (IPTG) & AppliChem GmbH, Darmstadt \\
\hline Kanamycin & Sigma-Aldrich, Steinheim \\
\hline Low fat dry milk & Regilait, Saint Martin Belle-Roche (F) \\
\hline Magnesium chloride $\left(\mathrm{MgCl}_{2}\right)$ & Merck, Mannheim \\
\hline Magnesium sulfate $\left(\mathrm{MgSO}_{4}\right)$ & Roth, Karlsruhe \\
\hline
\end{tabular}




\begin{tabular}{|c|c|}
\hline D-(+)-Maltose monohydrate & Sigma-Aldrich, Steinheim \\
\hline Manganese chloride $\left(\mathrm{MnCl}_{2}\right)$ & Roth, Karlsruhe \\
\hline Methanol & Sigma-Aldrich, Steinheim \\
\hline Nickel chloride $\left(\mathrm{NiCl}_{2}\right)$ & Riedel-de Haen,Seelze \\
\hline Nonidet P-40 (NP-40) & Roche, Penzberg \\
\hline Nystatin & AppliChem GmbH, Darmstadt \\
\hline N-Z-amine & Roth, Karlsruhe \\
\hline Peptone & Roth, Karlsruhe \\
\hline Phenol:Chlorofom:Isoamyl alcohol (25:24:1) & Sigma-Aldrich, Steinheim \\
\hline Phenylmethylsulfonylfluoride (PMSF) & Serva, Heidelberg \\
\hline Potassium chloride $(\mathrm{KCl})$ & Roth, Karlsruhe \\
\hline Potassium Dihydrogen phosphate $\left(\mathrm{KH}_{2} \mathrm{PO}_{4}\right)$ & Roth, Karlsruhe \\
\hline Sodium acetate & Roth, Karlsruhe \\
\hline Sodium azide $\left(\mathrm{NaN}_{3}\right)$ & Alfar Aesar, Karlsruhe \\
\hline Sodium dihydrogenphophate $\left(\mathrm{NaH}_{2} \mathrm{PO}_{4}\right)$ & Merck, Mannheim \\
\hline Sodium dodecyl sulfate (SDS) & VWR, Poole \\
\hline Sodium hydroxide $(\mathrm{NaOH})$ & Merck, Mannheim \\
\hline Sodium hypochlorite $(\mathrm{NaClO})$ & Roth, Karlsruhe \\
\hline Sodium molybdate $\left(\mathrm{Na}_{2} \mathrm{MoO}_{4}\right)$ & Merck, Mannheim \\
\hline Sodium monohydrogenphosphate $\left(\mathrm{Na}_{2} \mathrm{HPO}_{4}\right)$ & Merck, Mannheim \\
\hline Sodium selenite $\left(\mathrm{Na}_{2} \mathrm{SeO}_{3}\right)$ & Alfar Aesar, Karlsruhe \\
\hline Sodium sulfate $\left(\mathrm{Na}_{2} \mathrm{SO}_{4}\right)$ & Merck, Mannheim \\
\hline Soium chloride $(\mathrm{NaCl})$ & Merck, Mannheim \\
\hline Tetramethylethylenediamine (TEMED) & Sigma-Aldrich, Steinheim \\
\hline TriChloroacetic acid (TCA) & Merck, Mannheim \\
\hline Tris(hydroxymethyl)aminoethane (Tris) & Roth, Karlsruhe \\
\hline Triton ${ }^{\circledR} \mathrm{X}-100$ & Merck, Mannheim \\
\hline TRIzol $^{\mathbb{B}}$ Reagent & Invitrogen, Karlsruhe \\
\hline Tryptone & Roth, Karlsruhe \\
\hline Tween ${ }^{\circledR}-20$ & Sigma-Aldrich, Steinheim \\
\hline Yeast extract & MOBIO, Hamburg \\
\hline Zink sulfate $\left(\mathrm{ZnSO}_{4}\right)$ & AppliChem GmbH, Darmstadt \\
\hline$\alpha$-Lactose & Roth, Karlsruhe \\
\hline
\end{tabular}

\subsubsection{Consumables}

Generally used consumables and other reagents are listed in Table 2-3

Table 2-3: Generally used consumables and other reagents

\begin{tabular}{|ll|}
\hline Consumables / Others & Supplier \\
\hline \hline 9cm, $6 \mathrm{~cm}, 3.5 \mathrm{~cm}$ Petri dish & Greiner, Solingen \\
\hline Amersham Hyperfilm ECL $(18 \times 24 \mathrm{~cm})$ & GE Healthcare, Buckinhamshire (UK) \\
\hline Amicon Ultra-15 Centrifugal Filter Units & Millipore, Billerica (USA) \\
\hline Amylose Resin & New England Biolabs, Ibswich (USA) \\
\hline Cryotubes & Greiner, Solingen \\
\hline Dynabeads ${ }^{\circledR}$ M280 sheep anti mouse IgG & Invitrogen, Karlsruhe \\
\hline Dynabeads ${ }^{\circledR}$ M280 sheep anti rabbit IgG & Invitrogen, Karlsruhe \\
\hline Femotip ${ }^{\circledR} \mathrm{II}$ & Eppendorf, Hamburg \\
\hline GeneRuler ${ }^{\text {tm }} 1$ kb Plus DNA ladder & Fermentas, St. Leon-Rot \\
\hline Glutathione Sepharose 4 Fast Flow & GE Healthcare, Buckinhamshire (UK) \\
\hline
\end{tabular}




\begin{tabular}{|ll|}
\hline Microloader & Eppendorf, Hamburg \\
\hline Mono Q $^{\mathrm{TM}} 5 / 50 \mathrm{GL}$ & GE Healthcare, Buckinhamshire (UK) \\
\hline Ni-NTA resin & Qiagen, Hilden \\
\hline Nitrocellulose membrane & GE Healthcare, Buckinhamshire (UK) \\
\hline Phase Lock Gel $^{\mathrm{TM}}(2 \mathrm{ml}$, heavy) & Eppendorf, Hamburg \\
\hline Pierce $^{\circledR}$ Avidin Agarose Resin & Thermo Fisher Scientific, Rockford (USA) \\
\hline $\begin{array}{l}\text { Roche's EDTA-free Complete Protease Inhibitor } \\
\text { Cocktail }\end{array}$ & Roche, Penzberg \\
\hline SeeBlue ${ }^{\circledR}$ Plus2 Pre-Stained protein standard & Invitrogen, Karlsruhe \\
\hline Superdex 75 10/300 GL & GE Healthcare, Buckinhamshire (UK) \\
\hline Superdex 200 10/30 GL & GE Healthcare, Buckinhamshire (UK) \\
\hline Superose 6 30/100 GL & GE Healthcare, Buckinhamshire (UK) \\
\hline \hline
\end{tabular}

\subsubsection{Kits}

Generally used Kits are listed in Table 2-4

Table 2-4: generally used Kits

\begin{tabular}{|c|c|}
\hline Kits & Supplier \\
\hline 2x iQ SYBR ${ }^{\circledR}$ Green Supermix & BIO-RAD, Muenchen \\
\hline Coomassie Plus (Bradford) Protein Assay & Thermo Fisher Scientific, Rockford (USA) \\
\hline DNA Clean \& Concentrator ${ }^{\mathrm{TM}}-5$ & Zymo Research, Orange (USA) \\
\hline ECL (PLUS) Western Blotting Detection Reagents & GE Healthcare, Buckinhamshire (UK) \\
\hline Micro BSA Protein Assay Kit & Thermo Fisher Scientific, Rockford (USA) \\
\hline NucleoBond® Xtra Midi & Macherey \& Nagel, Dueren \\
\hline QIAprep ${ }^{\circledR}$ Spin Miniorep & Qiagen, Hilden \\
\hline $\begin{array}{l}\text { SuperScript }{ }^{\mathrm{tm}} \text { III First-Strand Synthesis System for } \\
\text { RT-PCR }\end{array}$ & Invitrogen, Karlsruhe \\
\hline $\begin{array}{l}\text { TNT }^{\circledR} \text { T7 Quick Coupled Transcription/ Translation } \\
\text { System }\end{array}$ & Promega, Madison (USA) \\
\hline Zymoclean $^{\mathrm{TM}}$ Gel DNA Recovery Kit & Zymo Research, Orange (USA) \\
\hline
\end{tabular}

\subsubsection{Enzymes}

Generally used enzymes are listed in Table 2-5

Table 2-5: Generally used enzymes

\begin{tabular}{|ll|}
\hline \hline Enzyme & Supplier \\
\hline \hline Antarctic phosphatase & New England Biolabs (NEB), Ibswich (USA) \\
\hline BamHI, NcoI, NdeI, NotI, SacII, StuI, XhoI & New England Biolabs (NEB), Ibswich (USA) \\
\hline DNaseI & New England Biolabs (NEB), Ibswich (USA) \\
\hline DpnI & New England Biolabs (NEB), Ibswich (USA) \\
\hline PfuUltra ${ }^{\mathrm{TM}}$ II Fusion HS DNA Polymerase & Stratagene, La Jolla (USA) \\
\hline Proteinase K & Invitrogen, Karlsruhe \\
\hline T4 DNA Ligase & New England Biolabs (NEB), Ibswich (USA) \\
\hline Taq DNA Polymerase (recombinant), LC & Fermentas, St. Leon-Rot \\
\hline \hline
\end{tabular}




\subsubsection{Histone peptides}

C-terminal biotinylated histone peptides were synthesized at the Griesinger Department at the Max Planck Institute of Biophysical Chemistry in Goettingen (MPI) or at the Rockefeller University Proteomics Core Facility in New York (USA) (RU). The obtained peptides were either unmodified or they contained specified mono-, di- or tri-methylated lysines and are listed in Table 2-6.

Table 2-6: Generally used biotinylated peptides

\begin{tabular}{|c|c|c|c|c|}
\hline "Name & Histone (aa) & Peptide sequence & "Modification & Supplier \\
\hline H3K4me3 & H3 (1-20) & ARTK $_{4}(\mathrm{me} 1) \mathrm{QTARKSTGGKAPRKQ}$ & K4me3 & RU \\
\hline H3K9me0 & H3 (1-20) & ARTK $_{4}$ QTARK $_{9}$ STGGKAPRKQ & unmodified & MPI \\
\hline H3K9me1 & H3 (1-20) & ARTKQTARK $_{9}($ me1)STGGKAPRKQ & K9me1 & MPI \\
\hline H3K9me2 & H3 (1-20) & ARTKQTARK $_{9}(\mathrm{me} 2)$ STGGKAPRKQ & K9me2 & MPI \\
\hline H3K9me3 & H3 (1-20) & ARTKQTARK $_{9}(\mathrm{me} 3)$ STGGKAPRKQ & K9me3 & MPI \\
\hline $\mathrm{H} 3 \mathrm{~K} 27 \mathrm{me} 0$ & H3 (19-38) & QLATKAARKSAPATGGVKKP & unmodified & RU \\
\hline H3K27me3 & H3 (19-38) & QLATKAARK $_{27}(\mathrm{me} 3)$ SAPATGGVKKP & K27me3 & RU \\
\hline H3K36me3 & H3 (27-46) & KSAPATGGVK $_{36}(\mathrm{me} 3) \mathrm{KPHRYRPGTV}$ & K36me3 & RU \\
\hline H3K64me0 & H3 (57-76) & STELLIRKLPFQRLVREIAQ & unmodified & RU \\
\hline H3K64me3 & H3 (57-76) & STELLIRK $_{64}(\mathrm{me} 3)$ LPFQRLVREIAQ & K64me3 & RU \\
\hline H4K20me0 & H4 (12-27) & KGGAKRHRKVLRDNIQ & unmodified & MPI \\
\hline H4K20me3 & H4 (12-27) & KGGAKRHRK $_{20}(\mathrm{me} 3)$ VLRDNIQ & K20me3 & MPI \\
\hline
\end{tabular}

\subsubsection{Antibodies obtained from different sources}

Generally used antibodies are listed in Table 2-7

Table 2-7: Generally used antibodies

\begin{tabular}{|llll|}
\hline \hline Name & Supplier; order number & Host & Dilution \\
\hline \hline Primary antibodies & & & \\
\hline$\alpha$ GFP & Roche, Mannheim; 11814460001 & Mouse, monoclonal & $1: 1000$ \\
\hline$\alpha$ GST & $\begin{array}{l}\text { Santa Cruz Biotechnology, Inc., } \\
\text { Santa Cruz (USA); sc - 138 }\end{array}$ & Mouse monoclonal & $1: 1000$ \\
\hline$\alpha H 3$ & Abcam, Cambridge (USA); ab1791 & Rabbit, polyclonal & $1: 10000$ \\
\hline$\alpha H$ PL-2 & Francesca Palladino [116] & Rabbit, polyclonal & $1: 1000$ \\
\hline$\alpha$ LIN-61 (guinea pig) & present study (2.4.1) & Guinea pig, polyclonal & $1: 1000$ \\
\hline$\alpha$ LIN-61 (rabbit) & present study (2.4.2) & Rabbit, polyclonal & $1: 4000$ \\
\hline$\alpha$ MBP tag & Santa Cruz Biotechnology, Inc., & Rabbit, polyclonal & $1: 1000$ \\
\hline$\alpha$ MYC tag & Santa Cruz (USA); sc - 808 & & \\
\hline \hline Secondary antibodies & Millipore, Billerica (USA), 05-724 & Mouse, monoclonal & $1: 1000$ \\
\hline \hline anti-guinea pig-HRP & DakoCytomation, Hamburg; P0141 & Rabbit, polyclonal & $1: 5000$ \\
\hline anti-mouse-HRP & DakoCytomation, Hamburg; P0447 & Goat, polyclonal & $1: 5000$ \\
\hline anti-rabbit-HRP & DakoCytomation, Hamburg; P0399 & Swine, polyclonal & $1: 5000$ \\
\hline \hline
\end{tabular}




\subsubsection{Primers}

Primer sequences were designed using the DNASTAR Lasergene 7 program and were ordered from MWG, Ebersberg. The primer sequences are listed in the method section when used (underlined sequences indicate restriction site, lower case indicate mutated nucleotide).

\subsubsection{Plasmids obtained from different sources}

Generally used plasmid vectors for cloning are listed in table Table 2-8.

Table 2-8: Generally used plasmid vectors

\begin{tabular}{|c|c|c|c|c|c|}
\hline Name & Type & Promoter & Selection & Tags & Source \\
\hline pETM-40 & $\begin{array}{l}\text { bacterial } \\
\text { expression } \\
\text { vector }\end{array}$ & T7 & Kanamycin & $\begin{array}{l}\text { MBP } \\
(\mathrm{N}-\text { term) }\end{array}$ & $\begin{array}{l}\text { G. Stier, EMBL Protein } \\
\text { expression and Purification } \\
\text { Facility, Heidelberg }\end{array}$ \\
\hline pET11a & $\begin{array}{l}\text { bacterial } \\
\text { expression } \\
\text { vector }\end{array}$ & $\mathrm{T} 7$ & Ampicillin & - & $\begin{array}{l}\text { New England Biolabs (NEB), } \\
\text { Ibswich (USA) }\end{array}$ \\
\hline pET16b & $\begin{array}{l}\text { bacterial } \\
\text { expression } \\
\text { vector }\end{array}$ & $\mathrm{T} 7$ & Ampicillin & $\begin{array}{l}\text { His6 } \\
\text { (N-term) }\end{array}$ & Merck, Darmstadt \\
\hline pCDNA3.1(+) & $\begin{array}{l}\text { mammalian } \\
\text { expression } \\
\text { vector }\end{array}$ & T7/CMV & Ampicillin & - & Invitogen, Karlsruhe \\
\hline pGEX-4T-3 & $\begin{array}{l}\text { bacterial } \\
\text { expression } \\
\text { vector }\end{array}$ & tac & Ampicillin & $\begin{array}{l}\text { GST } \\
(\mathrm{N}-\text { term })\end{array}$ & $\begin{array}{l}\text { GE Healthcare, } \\
\text { Buckinhamshire (UK) }\end{array}$ \\
\hline $\begin{array}{l}\text { pBluescript } \\
\text { SK(-) }\end{array}$ & $\begin{array}{l}\text { Phagemid } \\
\text { vector }\end{array}$ & $\mathrm{T} 7$ & Ampicillin & - & Stratagene, La Jolla (USA) \\
\hline L4440 & $\begin{array}{l}\text { Worm } \\
\text { expression, } \\
\text { RNAi }\end{array}$ & $\mathrm{T} 7$ & Ampicillin & - & $\begin{array}{l}\text { Andrew Fire, Stanford School } \\
\text { of Medicine (USA) }\end{array}$ \\
\hline
\end{tabular}

The plasmids that are listed in table Table 2-9 were obtained by isolation out of purchased bacterial clones.

Table 2-9: purchased plasmids containing either lin-61 or $13 m b t l 1$ cDNA

\begin{tabular}{|llllll|}
\hline \hline Name & insert & vector & clone ID & bacterial host & suppllier \\
\hline $\begin{array}{l}\text { R06C7.7 } \\
\text { pL4440-Dest }\end{array}$ & $\begin{array}{l}\text { lin-61 } \\
\text { cDNA }\end{array}$ & $\begin{array}{l}\text { pL4440- } \\
\text { Dest }\end{array}$ & $\begin{array}{l}\text { R06C7.7 CeRNAi } \\
\text { Feeder }\end{array}$ & $\begin{array}{l}\text { HT115(DE3) } \\
\text { (Fire lab) }\end{array}$ & $\begin{array}{l}\text { Thermo Fisher } \\
\text { Scientific, } \\
\text { Rockford (USA) }\end{array}$ \\
$\begin{array}{lllll}\text { 13mbtl1 pCMV- } \\
\text { SPORT6 }\end{array}$ & $\begin{array}{l}\text { 13mbtl1 } \\
\text { cDNA }\end{array}$ & $\begin{array}{l}\text { pCMV- } \\
\text { SPORT6 }\end{array}$ & BC039820.1 & DH10B & $\begin{array}{l}\text { Geneservice, } \\
\text { Cambridge (UK) }\end{array}$ \\
\hline \hline
\end{tabular}

The plasmid pRF4 was obtained from Dr. Monika Jedrusik-Bode (Max Planck Institute of biophysical Chemistry, Goettingen) and is described in [123]. 
In addition, the cosmid R06C7 (Z71266.1) that was originally generated in the Wellcome Trust Sanger Institue (Cambridge, UK) was obtained from Dr. Monika Jedrusik-Bode (Max Planck Institute of Biophysical Chemistry, Goettingen).

\subsubsection{Bacterial strains}

Bacterial strains used for cloning, expression of recombinant protein or as food source for C. elegans are listed in table Table 2-10

Table 2-10: Bacterial strains

\begin{tabular}{|c|c|c|c|}
\hline Strain & genotype / description & application & Source \\
\hline DH5a & $\begin{array}{l}\text { E. coli } \mathrm{F}-\mathrm{f} 80 \text { lacZDM15 } \mathrm{D}(\text { lacZYA-argF }) \mathrm{U} 169 \\
\text { deo } \mathrm{R} \text { recA1 end } 1 \text { } 16 \text { sd } 17\left(\mathrm{r}_{\mathrm{k}}^{-}, \mathrm{m}_{\mathrm{k}}^{+}\right) \text {phoAsup } 44 \mathrm{l}^{-} \\
\text {thi-1 gyrA96 relA1 }\end{array}$ & $\begin{array}{l}\text { plasmid } \\
\text { amplification; } \\
\text { cloning }\end{array}$ & $\begin{array}{l}\text { Invitrogen, } \\
\text { Karlsruhe }\end{array}$ \\
\hline $\begin{array}{l}\text { BL21- } \\
\text { CodonPlus(DE3)- } \\
\text { RIL }\end{array}$ & $\begin{array}{l}\text { E. coli } \mathrm{B} \mathrm{F}-\text { ompT hsdS }\left(\mathrm{r}_{\mathrm{B}}^{-} \mathrm{m}_{\mathrm{B}}^{-}\right) d c m+\mathrm{Tet}^{\mathrm{r}} \text { gal l } \\
\text { (DE3) endA Hte }\left[\arg U \text { ileY leuW } \mathrm{Cam}^{\mathrm{r}}\right]\end{array}$ & $\begin{array}{l}\text { protein } \\
\text { expression }\end{array}$ & $\begin{array}{l}\text { Stratagene, } \\
\text { La Jolla } \\
\text { (USA) }\end{array}$ \\
\hline OP50-1 & $\begin{array}{l}\text { Uracil auxotroph. E. coli B, streptomycin resistant, } \\
\text { useful for growing C. elegans in bulk }\end{array}$ & $\begin{array}{l}\text { C. elegans } \\
\text { food }\end{array}$ & CGC \\
\hline HB101 & $\begin{array}{l}\text { This strain of } E \text {. coli is easier for worms to eat than } \\
\text { other E. coli strains. [supE44 hsdS20(rB-mB-) } \\
\text { recA13 ara-14 proA } 2 \text { lacY } 1 \text { galK2 rpsL20 xyl-5 } \\
\text { mtl-1]. Contains a mutation (rpsL20) in a ribosomal } \\
\text { subunit gene that confers streptomycin resistance }\end{array}$ & $\begin{array}{l}\text { C. elegans } \\
\text { food }\end{array}$ & CGC \\
\hline HT115(DE3) & $\begin{array}{l}\text { E. coli } \mathrm{F}-\text {, mcrA, mcrB, IN(rrnD-rrnE)1, } \\
\text { rnc14::Tn10(DE3 lysogen: lavUV5 promoter -T7 } \\
\text { polymerase) (IPTG-inducible T7 polymerase) } \\
\text { (RNAse III minus), tetracycline resistant }\end{array}$ & $\begin{array}{l}\text { RNAi } \\
\text { feeding }\end{array}$ & CGC \\
\hline
\end{tabular}

(CGC stands for Caenorhabditis Genetics Center (CGC), which is funded by the NIH National Center for Research Resources (NCRR))

For RNAi feeding experiments, a HT115(DE3) bacteria strain was purchased from Open Biosystem (Thermo Fisher Scientific, Rockford (USA)) with the clone ID ZK678.1. This strain contains the lin-15A coding sequence cloned into the pl4440Dest vector.

\subsubsection{C. elegans strains obtained from different sources}

C. elegans strains obtained from different sources are listed in Table 2-11

Table 2-11: C. elegans strains obtained from different sources

\begin{tabular}{|c|c|c|c|}
\hline Strain & Genotype & Source & Reference \\
\hline $\mathrm{N} 2$ & wild-type & $\begin{array}{l}\text { Hodkin J, Oxford University, Oxford (UK); } \\
\text { distributed by the CGC }\end{array}$ & [124] \\
\hline PFR40 & hpl-2(tm1489)III & $\begin{array}{l}\text { Palladino F, Ecole Noramle Superieure de } \\
\text { Lyon, Lyon }(F) \text {; distributed by the CGC }\end{array}$ & [114] \\
\hline MT1806 & $\operatorname{lin}-15 A(n 767) X$ & $\begin{array}{l}\text { Horvitz R, Massachusetts Institute of } \\
\text { Technology, distributed by the CGC }\end{array}$ & [84] \\
\hline lin- & $\operatorname{lin}-61(\operatorname{tm} 2649) I$ & National BioResource Project NBRP, Mitani & - \\
\hline
\end{tabular}


Material and Methods

\begin{tabular}{|c|c|c|c|}
\hline $61(\operatorname{tm} 2649)$ & & $\begin{array}{l}\text { Laboratory at the Tokyo Women's Medical } \\
\text { University School of of Medicine }\end{array}$ & \\
\hline MT15643 & $m b t r-1(n 4775) I$ & $\begin{array}{l}\text { Horvitz R, Massachusetts Institute of } \\
\text { Technology }\end{array}$ & {$[100]$} \\
\hline MT13293 & $m e t-2(n 4256) I I I$ & $\begin{array}{l}\text { Horvitz R, Massachusetts Institute of } \\
\text { Technology }\end{array}$ & {$[112]$} \\
\hline GS2362 & $\operatorname{arIs} 48[G F P: \because$ lin-13] & Greenwald I., Howard Hughes Medical Institute & {$[118]$} \\
\hline
\end{tabular}

(CGC stands for Caenorhabditis Genetics Center (CGC), which is funded by the NIH National Center for Research Resources (NCRR))

Molecular details of the genotype of mutant C. elegans strains are listed in Table 2-12

Table 2-12: Molecular details of genotypes

\begin{tabular}{|c|c|}
\hline Genotype & Molecular details (taken from wormbase (http://www.wormbase.org/)) \\
\hline $\begin{array}{l}\text { hpl-2 } \\
(t m 1489)\end{array}$ & $\begin{array}{l}\text {...gagaataatcgaacaaaaatc [672 BP DELETION] aaaaatatggaataaagattc... -- Wild-type } \\
\text {...gagaataatcgaacaaaaatc - }\end{array}$ \\
\hline $\begin{array}{l}\operatorname{lin}-15 A \\
(n 767)\end{array}$ & 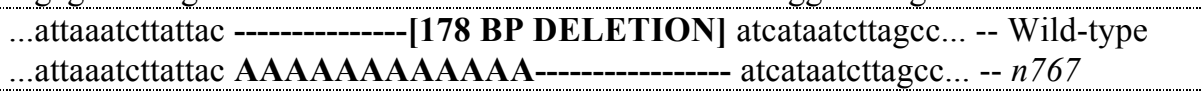 \\
\hline $\begin{array}{l}\operatorname{lin}-61 \\
(\operatorname{tm} 2649)\end{array}$ & $\begin{array}{l}\text {...ctcaatatattccttttcgc [672 BP DELETION] tgagccagaactctgtatccg... -- Wild-type } \\
\text {...ctcaatatattcetttttcgc - }\end{array}$ \\
\hline $\begin{array}{l}m b t r-1 \\
(n 4775)\end{array}$ & $\begin{array}{l}\text {...caattaatttaaaaattgag [1878 BP DELETION] aatttgttgaaaaataacct... -- Wild-type } \\
\text {...caattaatttaaaaattgag - }\end{array}$ \\
\hline $\begin{array}{l}\text { met-2 } \\
(n 4256)\end{array}$ & $\begin{array}{l}\text {...catgctcactctgattcgatt [1343 BP DELETION] attttcttcgacaacggaacc... -- Wild-type } \\
\text {....catgctcactctgattcgatt -------------- -- atttcttcgacaacggaacc... -- } n 4256\end{array}$ \\
\hline
\end{tabular}

Note: Sequence is reported on the plus strand

\subsection{Molecular biological methods}

\subsubsection{Plasmid DNA preparation}

Plasmid DNA was isolated from DH5 $\alpha$ bacteria bearing the desired plasmid that were cultured over night at $37^{\circ} \mathrm{C}$ in either $5 \mathrm{ml}$ or $100 \mathrm{ml} \mathrm{LB}$ media $(1 \% \mathrm{w} / \mathrm{v}$ peptone, $0.5 \%$ $\mathrm{w} / \mathrm{v}$ yeast extract, $0.5 \% \mathrm{w} / \mathrm{v} \mathrm{NaCl}$ ) using either the QIAprep ${ }^{\circledR}$ Spin Miniprep Kit (Qiagen) or the NucleoBond ${ }^{\circledR}$ Xtra Midi Kit (Macherey \& Nagel), according to manufacturer's protocol. The concentration of the isolated plasmid DNA was determined photometricaly at $260 \mathrm{~nm}$ using the NanoDrop ${ }^{\circledR}$ ND-1000.

\subsubsection{DNA digestion with restriction endonucleases}

DNA was digested with various restriction restriction endonucleases (New England Biolabs (NEB)) dependent on the DNA sequence and on the purpose of use, according to NEB protocols [125].

\subsubsection{Polymerase chain reaction (PCR)}

Amplification of particular DNA sequences defined by the used DNA template and the chosen primers was performed by PCR according to [126]. Unless otherwise 
specified, $50 \mu \mathrm{l}$ PCR reaction mix (50 ng template DNA, $500 \mathrm{nM}$ of each chosen primer, $200 \mu \mathrm{M}$ of each dNTP (100 mM dNTP Set, Invitrogen), 1 unit PfuUltra ${ }^{\mathrm{TM}}$ II Fusion HS DNA Polymerase (Stratagene), $5 \mu 1$ 10x reaction buffer provided by the manufacturer) was incubated in the PCR machine 'Eppendorf Mastercycler ep gradient $\mathrm{S}^{\prime}$ using the following program: $1 \mathrm{~min}$ at $94^{\circ} \mathrm{C}-\left[30 \mathrm{sec}\right.$ at $94^{\circ} \mathrm{C}-1 \mathrm{~min}$ at $55^{\circ} \mathrm{C}-1$ min per kb of amplicon length at $\left.72^{\circ} \mathrm{C}\right] 8 \mathrm{x}-\left[30 \mathrm{sec}\right.$ at $94^{\circ} \mathrm{C}-1 \mathrm{~min}$ at $60^{\circ} \mathrm{C}-$ 1 min per kb of amplicon length at $\left.72^{\circ} \mathrm{C}\right]_{27 \mathrm{x}}-10 \mathrm{~min}$ at $72^{\circ} \mathrm{C}$.

\subsubsection{Separation and isolation of DNA fragments}

To separate DNA fragments corresponding to size, agarose gel electrophoresis was performed according to standard protocols [126]. DNA solutions were mixed with 10x loading dye (10 mM EDTA, 30\% w/v glycerol, $100 \mu \mathrm{g} / \mathrm{ml}$ bromphenol blue), loaded into slots of $1 \%$ to $2 \%$ agarose gels $(1-2 \% \mathrm{w} / \mathrm{v}$ agarose, $89 \mathrm{mM}$ Tris, $89 \mathrm{mM}$ boric acid, $2 \mathrm{mM}$ EDTA, $0.01 \% \mathrm{v} / \mathrm{v}$ ethidium bromide, $\mathrm{pH} 8.0$ ) and run for $30 \mathrm{~min}$ at $120 \mathrm{~V}$ in TBE buffer ( $89 \mathrm{mM}$ Tris, $89 \mathrm{mM}$ boric acid, 2 mM EDTA, pH 8.0) using the Sub-Cell-GT Agarose gel electrophoresis system. The DNA was visualized using UV light $(320 \mathrm{~nm})$ as the fluorescence of ethidium bromide is strongly enhanced by the intercalation with DNA. In all runs, GeneRuler ${ }^{\mathrm{TM}} 1 \mathrm{~kb}$ Plus DNA Ladder was loaded in parallel to the samples as size reference. If desired, single DNA fragments were isolated out of the agarose gels using the Zymoclean ${ }^{\mathrm{TM}}$ Gel DNA Recovery Kit (Zymo Research), according to manufacturer's instructions.

In addition to the gel extraction method, DNA sequences were isolated and purified using the DNA Clean \& Concentrator ${ }^{\mathrm{TM}}-5$ Kit (Zymo Research), according to manufacturer's protocol, or by phenol/chloroform extraction.

To perform phenol/chloroform extraction, the DNA sample was mixed with an equal volume of Phenol:Chlorofom:Isoamyl alcohol (25:24:1) and centrifuged at 14000xg for $5 \mathrm{~min}$ in Phase Lock Gel ${ }^{\mathrm{TM}}(2 \mathrm{ml}$, heavy) tubes. The upper aqueous phase was mixed with an equal volume of chloroform:isoamyl alcohol (24:1) and the centrifugation step was repeated. The upper aqueous phase, containing the DNA was transferred to a new tube.

Ethanol precipitation was conducted by mixing the DNA solution with a 2.5 -fold volume of ethanol and 1/10 volume of $3 \mathrm{M}$ Sodium acetate $\mathrm{pH}$ 5.2. The mixture was incubated $2 \mathrm{hrs}$ to $\mathrm{ON}$ at $-20^{\circ} \mathrm{C}$ before it was centrifuged $\left(16000 \mathrm{xg}, 30 \mathrm{~min}, 4^{\circ} \mathrm{C}\right)$. The 
DNA pellet was washed with 75\% ethanol and after drying, the pellet was resolved in a small volume of $\mathrm{H}_{2} \mathrm{O}(20-50 \mu \mathrm{l})$.

\subsubsection{Transformation of plasmids into chemically competent bacteria}

To transfer plasmid DNA into DH5 $\alpha$ or B121-CodonPlus ${ }^{\circledR}(\mathrm{DE} 3)-(\mathrm{RIL})$ bacteria according to Sambrook et al. [126], chemically competent bacteria stored at $-80^{\circ} \mathrm{C}$ in $50 \mu 1$ aliquots were used. For transformation, an aliquot of bacteria was thawed on ice and incubated with the plasmid DNA of choice for $20 \mathrm{~min}$ on ice. The bacteria/DNA mixture was heat-shocked at $42^{\circ} \mathrm{C}$ for $1 \mathrm{~min}$ and afterwards cooled on ice for $2 \mathrm{~min}$ before $500 \mu \mathrm{l}$ of SOC medium $(2 \% \mathrm{w} / \mathrm{v}$ tryptone, $0.5 \% \mathrm{w} / \mathrm{v}$ yeast extract, $10 \mathrm{mM}$ $\mathrm{NaCl}, 2.5 \mathrm{mM} \mathrm{KCl}, 10 \mathrm{mM} \mathrm{MgCl} 2,20 \mathrm{mM}$ glucose) was added. The mixture was incubated at $37^{\circ} \mathrm{C}$ while shaking for $60 \mathrm{~min}$ and subsequently centrifuged at low speed (1500xg) for $2 \mathrm{~min}$. Most of the supernatant was removed by aspiration, and the bacteria pellet was resuspended in remaining media and streaked on an LB-plate (1\% $\mathrm{w} / \mathrm{v}$ tryptone, $0.5 \%$ yeast extract, $1 \% \mathrm{w} / \mathrm{v} \mathrm{NaCl}, 1.5 \%$ agar) containing the required antibiotics (100 $\mu \mathrm{g} / \mathrm{ml}$ ampicillin or $50 \mu \mathrm{g} / \mathrm{ml}$ kanamycin) for incubation $\mathrm{ON}$ at $37^{\circ} \mathrm{C}$. Single grown bacterial colonies were used to inoculate growth media dependent on the purpose of use.

\subsubsection{General cloning procedure}

In general, cloning of cDNA or genomic DNA was performed according to Sambrook et al. [126]. The DNA sequence was amplified by PCR (2.2.3) using specific primers flanking the desired DNA sequences and containing specific restriction endonuclease cutting sites. The PCR amplicon was purified and the PCR amplicon and the target vector were digested with specific restriction endonucleases $(2.2 .2,2.2 .4)$. The digested DNAs were separated on agarose gels and purified by gel extraction (2.2.4). The vector was treated with Antarctic phosphatase (NEB), according to the manufacturer's protocol [125]. $100 \mathrm{ng}$ of dephosphorylated vector were incubated ON at $15^{\circ} \mathrm{C}$ with a 3 -fold molar excess of PCR amplicon and ligated using $1 \mu 1 \mathrm{~T} 4 \mathrm{DNA}$ ligase (NEB) in a reaction volume of $20 \mu \mathrm{l}$ including $2 \mu \mathrm{l} 10 \mathrm{x}$ reaction buffer provided by the manufacturer. $10 \mu 1$ of the ligation reaction were transformed into DH5 $\alpha$. For plasmid preparation, several single colonies were selected to inoculate 5 $\mathrm{ml}$ LB media cultures (2.2.5). Plasmid DNA was isolated from these cultures (2.2.1), 
and aliquots of $1 \mu \mathrm{g}$ were sequenced (MWG (Ebersberg) or Seqlab (Göttingen)). Plasmids containing the desired correct insert were kept.

\subsubsection{Site-directed mutagenesis}

Site-directed mutagenesis of plasmid DNA was performed according to the 'QuickChange Site-directed mutagenesis' protocol. Two primers of complementary sequence were designed containing the desired nucleotide exchanges. The mutated nucleotides were located in the middle of the primers with at least 15 bases up- and downstream. A PCR reaction was performed using the original plasmid as template and the resulting PCR product was purified $(2.2 .3,2.2 .4)$. To eliminate template plasmid DNA, the purified DNA solution was digested with DpnI (NEB) for $60 \mathrm{~min}$ at $37^{\circ} \mathrm{C}$ according to the manufacturer's protocol. The remaining plasmid DNA was transformed into DH5 $\alpha$.

\subsubsection{Generated plasmid constructs}

\subsubsection{1 pETM40 expression vector based constructs}

The DNA sequence encoding LIN-61 (gi: 193203072) was amplified from the R06C7.7 pL4440-Dest plasmid by PCR and cloned into the pETM40 vector via NcoI and XhoI restriction sites to generate a construct encoding MBP-tagged LIN-61 (2.2.6). The following primers were used:

\footnotetext{
lin61AscNco-f: 5'-GAAAGGCGCGCCACCATGGGAATGTCTGAATTTCTGAAAATTCTC-3' lin61BamXho-r: 5'-CAGGTTGGATCCCTCGAGTCAATAGTTGTACTTTTTCGGAGG-3，
}

\subsubsection{2 pcDNA3.1 $(+)$ mammalian expression vector based constructs}

To generate a construct encoding MYC-tagged LIN-61, the DNA sequence encoding a MYC-tag followed by a NotI restriction site was directly fused 5' to the lin-61 coding sequence by PCR (2.2.3). The PCR amplicon was cloned into pcDNA3.1(+) via BamHI and XhoI restriction sites (2.2.6). The following primers were used:

\begin{tabular}{|c|c|}
\hline \multirow[t]{2}{*}{ BamHINtermmyclin61f: } & 5'-GAGAGGCGGGATCCACCATGGAACAAAAACTCATCTCAG \\
\hline & AAGAGGATCTGAATGCGGCCGCAATGTCTGAATTTCTG-3 ' \\
\hline lin-61-XhoI-rev: & 5'-CAGGTTCACTCGAGTCAATAGTTGTACTTTTTCGGAGG-3' \\
\hline
\end{tabular}

Within the pcDNA3.1(+)-myc-lin-61 construct (see above) point mutations were introduced by site-directed mutagenesis (2.2.7). 
To generate a construct encoding MYC-tagged LIN-61 D428A point mutant protein, the following primers were used:

$\begin{array}{ll}\text { lin-61 da for: } & \text { 5'-GATTTTCACACATGgCAGCAGCTTCAAGGCGTAG-3' } \\ \text { lin-61 da rev: } & 5 \text { '-CTTGAAGCTGCTGCCATGTGTGAAAATCAGTTTATTTGTC-3', }\end{array}$

To generate a construct encoding MYC-tagged LIN-61 F452A W455A F459A triple point mutant protein, the following primers were used:

lin-61 cage for:

5'-CCACATCATACAGTTCATCAgcTTCTTCATCCgcGCCGTCGgc ATTGACATTTATCAGTC-3,

lin-61 cage rev: 5'-GATAAATGTCAATgcCGACGGCgcGGATGAAGAAgcTGA TGAACTGTATGATGTGGACTC-3,

To generate constructs encoding MYC-tagged LIN-61 F452A, W455A or F459A single point mutant protein, the following primers were used:

LIN-61 F452A:

lmuta 1 fa for: 5'-CTTCATCCCAGCCGTCGgcATTGACATTTATCAGTCTTCC-3'

lmuta lfa rev: 5'-GACTGATAAATGTCAATgcCGACGGCTGGGATGAAGAATTTG-3'

LIN-61 W455A:

lmuta 2 wa for: 5'-CATCAAATTCTTCATCCgcGCCGTCGAAATTGACATTTATC-3'

lmuta 2wa rev: 5'-GTCAATTTCGACGGCgcGGATGAAGAATTTGATGAACTGTATG-3'

LIN-61 F459A:

lmuta 3 fa for:

5'-CATCATACAGTTCATCAgcTTCTTCATCCCAGCCGTCG-3' lmuta 3 fa rev:

5'-CGGCTGGGATGAAGAAgcTGATGAACTGTATGATGTGG-3'

To generate constructs encoding MYC-tagged LIN-61 single point mutant proteins in which the coding sequence of lin-61 corresponds to the coding lin-61 sequence of the lin-61(n3624) [LIN-61 P123S], the lin-61(n3736) [LIN-61 F247S], the lin-61(n3807) [LIN-61 G250E], the lin-61(n3447) [LIN-61 S354N] or the lin-61(n3922) [LIN-61 G445R] alleles, the following primers were used:

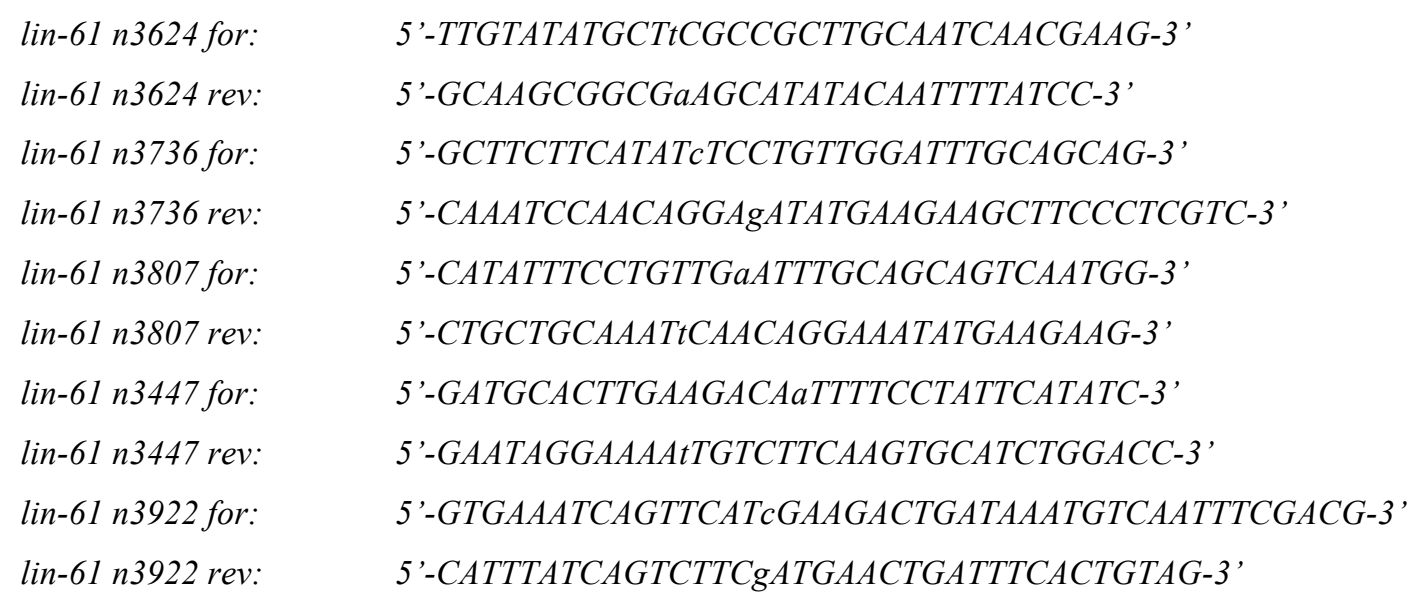


To generate a construct encoding a MYC-tagged MBTR-1 protein, the $m b t r-1$ coding sequence (gi: 114786042) was amplified by RT-PCR (2.5.12) from isolated $C$. elegans wild-type N2 total RNA. By cloning the mbtr-1 coding sequence via NotI and XhoI into the pcDNA3.1(+)-myc-lin-61 construct (see above), the lin-61 coding sequence was exchanged by the $m b t r-1$ coding sequence. The following primers were used:

mbtr-1 NotI for ains: Y48G_r_XhoI+stop:

$$
\begin{aligned}
& \text { 5'-GAGAGGCG } \underline{\text { ' } G G C C G C A A T G G A A A A A T C G T C G T C G-3} \text { ' } \\
& \text { 5'-GCG } \underline{C T C G A G T C A A T C G T A T T C T C C G T T T T C G G C G T T C-3} \text {, }
\end{aligned}
$$

\subsubsection{3 pGEX-4T-3 expression vector based constructs:}

To generate constructs encoding GST-tagged deletion proteins of LIN-61 containing three, two or one out of four MBT repeats, the corresponding DNA sequences were amplified by PCR and cloned into pGEX-4T3 via BamHI and XhoI restriction sites (2.2.6).

For cloning the DNA sequence encoding the LIN-61 MBT repeats two to four (amino acids 138 to 491 ) the following primers were used:

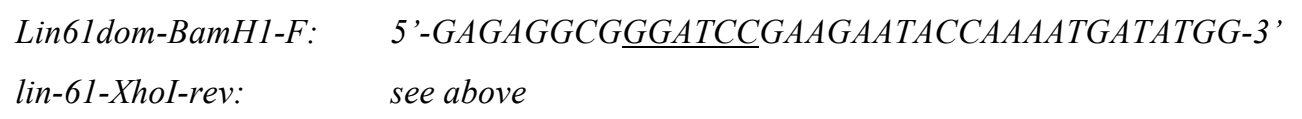

For cloning the DNA sequence encoding the LIN-61 MBT repeats two and three (amino acids 138 to 383 ) the following primers were used:

Lin61dom-BamH1-F: $\quad$ see above

lin61dom2st xhor: 5'-CAGGTTCA

For cloning the DNA sequence encoding the LIN-61 MBT repeats three and four (amino acids 266 to 491 ) the following primers were used:

lin61dom2bamfor: 5'-GAGAGGCGGGATCCATTGAGCACACAAATAAAATTGC-3,

lin-61-Xhol-rev: see above

For cloning the DNA sequence encoding the LIN-61 MBT repeat two (amino acids 138 to 265$)$ the following primers were used:

Lin61dom-BamH1-F: see above

lin61dom1stxhore: 5'-CAGGTTCACTCGAGTCAATATTCCTTTTTCGCATTTAG-3'

For cloning the DNA sequence encoding the LIN-61 MBT repeat three (amino acids 266 to 383) the following primers were used:

lin61dom2bamfor: see above

lin61dom2st xhor: see above

For cloning the DNA sequence encoding the LIN-61 MBT repeat four (amino acids 384 to 491) the following primers were used: 
Material and Methods

$\begin{array}{ll}\text { lin61dom3bamfor: } & \text { 5'-GAGAGGCGGGATCCAAAGGAACATTCAGATGGGATG-3' } \\ \text { lin-61-XhoI-rev: } & \text { see above }\end{array}$

\subsubsection{4 pET11a expression vector based construct}

The DNA sequence encoding LIN-61 was amplified by PCR and cloned into the pET11a vector via NdeI and BamHI restriction sites (2.2.6). The following primers were used:

$\begin{array}{ll}\text { Lin61NDEI forw: } & \text { 5'-CACTCGAGCATATGTCTGAATTTCTGAAAATTGTCAGAG-3' } \\ \text { lin61 BAMHI rev: } & \text { 5'-CACAGGTTGGATCCTCAATAGTTGTACTTTTTCGGAGGTTG-3' }\end{array}$

\subsubsection{5 pET16b expression vector based construct}

DNA sequences encoding the three MBT repeats of human L3MBTL1 were amplified by PCR and cloned into pET16b via NdeI and NotI restriction sites by Christoph Biesemann (rotation student of IMPRS Molecular Biology Program 2006) (2.2.6). For the PCR reaction, the full-length cDNA plasmid 13mbtl1 pCMV-SPORT6 was used as template with the following primers:
L3MBTL_NdeI_for:
5'-GCG CATATGCCTGCAACAGGTGAGAAGAAGGAATG-3'
L3MBTL_BamHI_r:

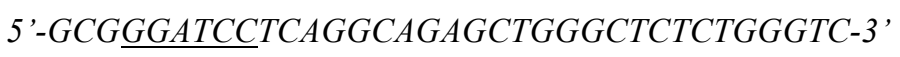

\subsubsection{6 pBluescript SK(-) vector based constructs}

A 'StuI - SacII' fragment of genomic C. elegans DNA (4387 bp) comprising the lin61 gene (R06C7.7) as the only complete predicted open reading frame (Figure 2-1) was amplified by PCR from the cosmid R06C7 (gi: 1279324) with the following primers:

$\begin{array}{ll}\text { vor stu 1 for: } & \text { 5'-CATTCTTCTTCCGGCAGAATCATCCAGTG-3' } \\ \text { Sac2 rev: } & 5 \text { '-CTTCATTTCCTCCGCGGTCATCATTTG-3' }\end{array}$

For cloning the genomic DNA fragment into pBluescript SK(-), the PCR amplicon was digested using StuI and SacII restriction enzymes. The vector was digested with SmaI and SacII restriction enzymes (2.2.6).

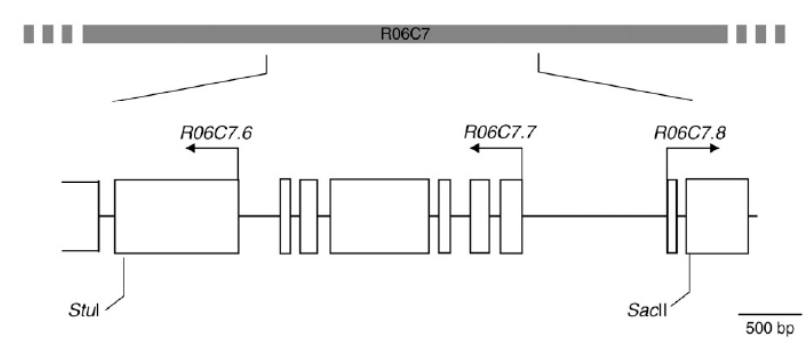

Figure 2-1: Schematic overview of StuI - SacII fragment of R06C7

Part of cosmid R06C7 is shown as shaded bar. The StuI- SacII fragment of R06C7 is shown below. Taken from [100] 
Within the pBluescript SK(-)-genomic-lin-61 construct (see above), three point mutations were introduced by site-directed mutagenesis to generate a construct in which the coding lin-61 sequence encodes a LIN-61 F452A W455A F459A triple point mutant protein (2.2.7). The site-directed mutagenesis was performed in two steps and the following primers were used:

lmuta 3 fa for: see above

lmuta 3fa rev: $\quad$ see above

lmuta3ara for: 5'-CATCAgcTTCTTCATCCgcGCCGTCGgcATTGACATTTATCAGTCTTC-3'

lmuta3ara rev: 5'-GACTGATAAATGTCAATgcCGACGGCgcGGATGAAGAAgcTGATGAAC-3'

\subsection{Protein biochemical methods}

\subsubsection{SDS polyacrylamide gel electrophoresis (SDS-PAGE)}

SDS-PAGE, based on the method from Laemmli [127], was carried out according to standard protocols [128] and used to separate proteins according to their molecular weight. Pouring and running of SDS-gels was carried out using the Mini-PROTEAN electrophoresis system. In general, Tris-glycine gels with a 10, 12 or $15 \%$ separating gel (10 - 15\% acrylamide/bisacrylamide (37.1:1), $0.4 \mathrm{M}$ Tris, 0.1\% w/v SDS, 5\% v/v glycerol, 0.1\% w/v APS, 0.04\% v/v TEMED, $\mathrm{pH} 8.8)$ and a 4\% stacking gel (4\% acrylamide:bisacrylamide (37.1:1), 0.68\% Tris, $0.1 \% \mathrm{w} / \mathrm{v}$ SDS, $0.1 \% \mathrm{w} / \mathrm{v}$ APS, $0.1 \%$ v/v TEMED, $\mathrm{pH}$ 6.8) were used. Protein samples were boiled for $5 \mathrm{~min}$ in protein sample buffer $(62.5 \mathrm{mM}$ Tris, 8.5\% v/v glycerol, 2\% w/v SDS, $100 \mu \mathrm{g} / \mathrm{ml}$ bromphenol blue, $150 \mathrm{mM}$ 2-mercaptoethanol), before loadung onto the gel. SDS-gels were typically run at constant voltage of $150 \mathrm{~V}$ in SDS-PAGE running buffer $(25 \mathrm{mM}$ Tris, $200 \mathrm{mM}$ glycine, $0.1 \% \mathrm{w} / \mathrm{v}$ SDS) until the blue running front reached the end of the gel. As size reference, $5 \mu$ of SeeBlue ${ }^{\circledR}$ Plus2 Pre-Stained protein standard was run in parallel to the samples.

For samples intended to be analyzed by mass spectrometry, the NuPAGE ${ }^{\circledR}$ Pre-cast system (Invitrogen) was used, according to manufacturer's instructions.

\subsubsection{Protein detection techniques}

\subsubsection{Coomassie Blue staining}

To stain proteins on SDS-PAGE gels, the gels were incubated in Coomassie staining solution $(0.05 \% \mathrm{w} / \mathrm{v}$ Coomassie brilliant blue, $10 \% \mathrm{v} / \mathrm{v}$ acetic acid, $50 \% \mathrm{v} / \mathrm{v}$ 
methanol) for $30 \mathrm{~min}$ with gentle rocking. Gels were destained with destaining solution $(10 \% \mathrm{v} / \mathrm{v}$ acetic acid, $7.5 \% \mathrm{v} / \mathrm{v}$ methanol) for at least $60 \mathrm{~min}$ to remove background staining.

\subsubsection{Western blotting}

To specifically detect proteins by interaction with specific antibodies, western blotting was performed. In general, proteins separated by SDS-PAGE (2.3.1) were transferred to nitrocellulose membrane in transfer buffer (25 mM Tris, $200 \mathrm{mM}$ glycine, 20\% v/v methanol, $0.1 \% \mathrm{w} / \mathrm{v} \mathrm{SDS}$ ) for $60 \mathrm{~min}$ at $4^{\circ} \mathrm{C}$ at constant voltage of $100 \mathrm{~V}$ using the Mini-Trans-Blot blot system. The nitrocellulose membrane was incubated in PBS-T milk (137 mM NaCl, $2.7 \mathrm{mM} \mathrm{KCl,} 10 \mathrm{mM} \mathrm{Na} \mathrm{HPO}_{4}, 2 \mathrm{mM} \mathrm{KH} \mathrm{KO}_{4}, 0.1 \% \mathrm{v} / \mathrm{v}$ Tween $^{\circledR}-20,5 \% \mathrm{w} / \mathrm{v}$ low-fat dry milk, $\mathrm{pH}$ 7.4) for $60 \mathrm{~min}$ while gentle rocking to block the membrane. Primary antibodies were diluted in PBS-T milk (dilutions see 2.1.7) and incubated with the membrane for $60 \mathrm{~min}$ at $\mathrm{RT}$ or $\mathrm{ON}$ at $4^{\circ} \mathrm{C}$. Then, the membrane was washed four times for 15 min each in PBS-T (same as PBS-T milk, but without milk) and subsequently incubated with the corresponding horseradish peroxidase (HRP) conjugated secondary antibodies diluted 1:5000 in PBS-T milk for $60 \mathrm{~min}$ at RT. Afterwards, the membrane was washed again four times for $15 \mathrm{~min}$ each in PBS-T before the antibody bound proteins were visualized on the membrane using ECL (Plus) Western Blotting Detection Reagents (GE Healthcare) and Amersham Hyperfilm ECL films (GE Healthcare) according to manufacturer's protocol. Film development was performed using the Kodak X OMAT 2000 processor.

\subsubsection{Mass spectrometry and analysis of results}

Mass spectrometry (MS) analysis was performed by the mass spectrometry group of Dr. Henning Urlaub at the Max Planck Institute of biophysical Chemistry (Göttingen), Briefly, after the protein sample was run on NuPAGE ${ }^{\circledR}$ gels (2.3.1), the gels were cut into 23 slices of equal size after Coomassie staining (2.3.2). Proteins within the slices were digested according to Shevchenko et al. [129] and peptides were extracted and analyzed by LC-coupled tandem MS on an Orbitrap XL mass spectrometer (Thermo Fisher Scientific). CID fragment spectra were searched against NCBInr database using MASCOT (with the taxonomy filter C. elegans) as search engine. Output files 
were subtracted according to the gi-numbers (NCBI) of the found proteins with the help of the statistical program $\mathrm{R}$ (for details of programming see 6.1).

\subsubsection{Expression of recombinant proteins in E. coli}

For expression of recombinant protein, a single colony of E. coli B121CodonPlus $^{\circledR}(\mathrm{DE} 3)-(\mathrm{RIL})$ cells, transformed with the corresponding coding plasmid (2.2.5), was used to inoculate $100 \mathrm{ml} \mathrm{LB}$ media. This starter culture was grown $\mathrm{ON}$ at $37^{\circ} \mathrm{C}$ while shaking $(130 \mathrm{rpm})$. It was used to inoculate a main culture in a ratio of $1 / 100$ the following morning. In all cases with the exception of expression of the HIS $_{6}$-tagged three MBT repeats of human L3MBTL1, ZYM-5052 auto-inducing medium $(1 \% \mathrm{w} / \mathrm{v} \mathrm{N}-\mathrm{Z}$-amine, $0.5 \% \mathrm{w} / \mathrm{v}$ yeast extract, $0.5 \% \mathrm{w} / \mathrm{v}$ glycerol, $0.05 \% \mathrm{w} / \mathrm{v}$ glucose, $0.2 \%$ w/v $\alpha$-lactose, $25 \mathrm{mM} \mathrm{Na}_{2} \mathrm{HPO}_{4}, 25 \mathrm{mM} \mathrm{KH}_{2} \mathrm{PO}_{4}, 50 \mathrm{mM} \mathrm{NH} \mathrm{Cl}_{4}, 5$ $\mathrm{mM} \mathrm{Na} \mathrm{SO}_{4}, 2 \mathrm{mM} \mathrm{MgCl} 2,50 \mu \mathrm{M} \mathrm{FeCl}_{3}, 20 \mu \mathrm{M} \mathrm{CaCl}_{2}, 10 \mu \mathrm{M} \mathrm{MnCl}, 10 \mu \mathrm{M}$ $\mathrm{ZnSO}_{4}, 2 \mu \mathrm{M} \mathrm{CoCl}_{2}, 2 \mu \mathrm{M} \mathrm{CuCl}_{2}, 2 \mu \mathrm{M} \mathrm{NiCl}_{2}, 2 \mu \mathrm{M} \mathrm{Na}_{2} \mathrm{MoO}_{4}, 2 \mu \mathrm{M} \mathrm{Na}_{2} \mathrm{SeO}_{3}, 2$ $\mu \mathrm{M} \mathrm{H}_{3} \mathrm{BO}_{3}$, either $100 \mu \mathrm{g} / \mathrm{ml}$ ampicillin or $50 \mu \mathrm{g} / \mathrm{ml}$ kanamycin dependent of the plasmid) was used. Cultures were grown for 4 days at $15^{\circ} \mathrm{C}$ while shaking $(120 \mathrm{rpm})$. Expression of the recombinant proteins was induced automatically in this medium by the presence of $\alpha$-lactose.

To express the $\mathrm{HIS}_{6}$-tagged three MBT repeats of human L3MBTL1, 2YT media ( $1.6 \%$ tryptone, $1 \%$ yeast extract, $0.5 \% \mathrm{NaCl}, 100 \mu \mathrm{g} / \mathrm{ml}$ ampicillin) was used. Cultures were shaken at $37^{\circ} \mathrm{C}$ until an $\mathrm{OD}_{600 \mathrm{~nm}}$ of $0.4-0.7$ was reached. At this time, the temperature was lowered to $25^{\circ} \mathrm{C}$ and the expression of the recombinant protein was induced by supplementing the culture with $0.2 \mathrm{mM}$ IPTG. The culture was grown for an additional $4-7 \mathrm{hrs}$.

Bacteria were harvested by centrifugation $\left(6000 \mathrm{xg}, 15 \mathrm{~min}, 4^{\circ} \mathrm{C}\right)$. Bacterial pellets were resuspended in $10 \mathrm{ml}$ lysis buffer (composition dependent on purpose of use) per 11 main culture and stored at $-80^{\circ} \mathrm{C}$ until further purification.

\subsubsection{Purification of recombinant proteins}

To purify recombinant proteins, the bacterial suspension (2.3.4) was thawed at $4{ }^{\circ} \mathrm{C}$ and the cells were lysed using an EmulsiFlex-C5 High Pressure Homogenizer (Avestin) at $4^{\circ} \mathrm{C}$, according to manufacturer's instructions. Afterwards, NP-40 was added to a final concentration of $0.5 \%$, and the suspension was stirred for $30 \mathrm{~min}$. Subsequently, the suspension was centrifuged $\left(20000 \mathrm{xg}, 45 \mathrm{~min}, 4^{\circ} \mathrm{C}\right)$ to remove 
insoluble cell material and the supernatant was kept. Further purification steps are described in the following sections.

\subsubsection{Purification of HIS $_{6}$-tagged recombinant proteins}

\section{Ni-NTA purification}

For purification of $\mathrm{HIS}_{6}$-tagged recombinant proteins, the bacterial pellets (2.3.4) were resuspended in Ni-NTA-lysis buffer ( $50 \mathrm{mM}$ sodium phosphate, $300 \mathrm{mM} \mathrm{NaCl}$, $10 \mathrm{mM}$ imidazole, $2 \mathrm{mM}$ PMSF, $2 \mathrm{mM}$ benzamidine, $10 \mathrm{mM}$ 2-mercaptoethanol, $\mathrm{pH}$ 8.0) and then handled as described above.

The supernatant was applied to Ni-NTA resin (nickel-loaded nitrilotriacetic acid coated agarose, Qiagen) that was packed into a plastic gravity column and was equilibrated with Ni-NTA-lysis buffer beforehand. The washing and elution procedure was carried out as described in the QIAexpressionist manual [130]. Briefly, the resin was washed with 100x column volumes wash buffer $(50 \mathrm{mM}$ sodium phosphate, $500 \mathrm{mM} \mathrm{NaCl}, 10 \mathrm{mM}$ imidazole, $2 \mathrm{mM}$ PMSF, $2 \mathrm{mM}$ benzamidine, 10 $\mathrm{mM}$ 2-mercatoethanol, $\mathrm{pH}$ 8.0) and $\mathrm{HIS}_{6}$-tagged protein was eluted with elution buffer (50 mM sodium phosphate, $100 \mathrm{mM} \mathrm{NaCl}, 250 \mathrm{mM}$ imidazole, $2 \mathrm{mM}$ PMSF, 2 $\mathrm{mM}$ benzamidine, $10 \mathrm{mM}$ 2-mercaptoethanol, $\mathrm{pH}$ 8.0) by gravity flow.

\section{Anion exchange chromatography}

To further purify proteins on a MonoQ ${ }^{\mathrm{TM}}$ 5/50 GL Anion exchange column (GE Healthcare), proteins were dialyzed to Mono-Q buffer 5\% B (25 mM Tris, $50 \mathrm{mM}$ $\mathrm{NaCl}, 5 \mathrm{mM}$ DTT, $\mathrm{pH}$ 8.0). The ion-exchange chromatography run was performed according to manufacturer's instructions on an ÄKTA Explorer FPLC instrument (GE Healthcare) with Mono-Q buffer A (25 mM Tris, $5 \mathrm{mM}$ DTT, pH 8.0) and Mono-Q buffer B (25 mM Tris, $1 \mathrm{M} \mathrm{NaCl}, 5 \mathrm{mM}$ DTT, pH 8.0) using the following protocol: 5 loading volumes 5\% Mono-Q buffer B - gradient to $60 \%$ buffer B in 20 column volumes -5 column volumes $100 \%$ Mono-Q buffer B. Peak fractions containing the recombinant protein were pooled and dialyzed to Mono-Q buffer A.

\section{Gel filtration chromatography}

In a final purification step, recombinant proteins were purified by gel filtration chromatography on a Superdex 75 10/300 GL column (GE Healthcare) according to manufacturer's instructions using Mono-Q buffer A to equilibrate the column. The 
chromatography was developed in Mono-Q buffer A and eluted fractions of recombinant protein were combined and concentrated using Amicon Ultra-15 Centrifugal Filter Units. The concentrated protein was stored at $4^{\circ} \mathrm{C}$.

\subsubsection{Purification of MBP-tagged proteins}

\section{Amylose bead purification}

For purification of MBP-tagged recombinant proteins, the bacterial pellets (2.3.4) were resuspended in MBP buffer (50 mM Tris, $200 \mathrm{mM} \mathrm{NaCl}, 1 \mathrm{mM}$ EDTA, $1 \mathrm{mM}$ DTT, 1mM PMSF, $1 \mathrm{mM}$ Benzamidine, pH 7.4) and then handled as described above. The supernatant was combined with amylose resin $(2 \mathrm{ml}$ resin per 11 culture volume; NEB) equilibrated in MBP buffer. The mixture was rotated $\mathrm{ON}$ at $4^{\circ} \mathrm{C}$. The beads were washed six times by incubating the beads for $30 \mathrm{~min}$ in ten-fold resin volume each with MBP buffer at $4^{\circ} \mathrm{C}$ followed by centrifugation at $500 \mathrm{xg}$ for $5 \mathrm{~min}$ at $4^{\circ} \mathrm{C}$. The MBP-tagged protein was eluted by incubation of the protein bound resin four times for $30 \mathrm{~min}$ each with MBP-buffer containing $10 \mathrm{mM}$ maltose. The eluted fractions containing the recombinant protein were combined and stored at $4^{\circ} \mathrm{C}$ until further use.

\section{Gel filtration chromatography}

To separate different subspecies of MBP-tagged full length LIN-61 dependent on its multimerization/aggregation state, gel filtration chromatography was performed after amylose bead purification. For this, the protein was dialyzed to $50 \mathrm{mM}$ Tris, $100 \mathrm{mM}$ $\mathrm{NaCl}, 1 \mathrm{mM}$ EDTA and concentrated using Amicon Ultra-15 Centrifugal Filter Units to $46 \mathrm{mg} / \mathrm{ml}$. For gel filtration $250 \mu \mathrm{l}$ of the protein sample was loaded on an equilibrated Superdex 200 10/300 GL column (GE Healthcare). The run was performed according to manufacturer's instructions using GF-buffer $(50 \mathrm{mM} \mathrm{Na}$ phosphate buffer $\mathrm{pH} 7.4,100 \mathrm{mM} \mathrm{NaCl}, 1 \mathrm{mM}$ EDTA). The eluted material was collected in $1 \mathrm{ml}$ fractions.

\subsubsection{Purification of GST-tagged proteins}

For purification of GST-tagged recombinant proteins, the bacterial pellets produced (2.3.4) were resuspended in GST-buffer ( $50 \mathrm{mM}$ Tris, $150 \mathrm{mM} \mathrm{NaCl}, 1 \mathrm{mM}$ EDTA, 1 mM DTT, 2 mM PMSF, 2 mM benzamidine) and then handled as described above. 
The supernatant was added to Glutathione Sepharose 4 Fast Flow resin $(1 \mathrm{ml}$ per 11 culture volume, GE Healthcare) equilibrated in GST-buffer in a test tube and incubated with the resin while rotating $\mathrm{ON}$ at $4^{\circ} \mathrm{C}$. The beads were washed six times by incubating the beads for $30 \mathrm{~min}$ in GST-buffer at $4^{\circ} \mathrm{C}$ followed by centrifugation at $500 \mathrm{xg}$ for $5 \mathrm{~min}$ at $4^{\circ} \mathrm{C}$. The GST-tagged protein was eluted from the beads by incubation of the resin four times / $30 \mathrm{~min}$ in two resin volumes of GST elution-buffer (50 mM Tris, $10 \mathrm{mM}$ gluthatione, $\mathrm{pH}$ 9.5). The eluted fractions containing the recombinant protein were combined and stored at $4^{\circ} \mathrm{C}$ until further use.

\subsubsection{Expression of MYC-tagged recombinant proteins in rabbit reticulocyte lysate}

MYC-tagged recombinant proteins were expressed using the $\mathrm{TNT}^{\circledR} \mathrm{T} 7$ Quick Coupled Transcription/Translation System (Promega) according to the manufacturer's protocol. In general, $17 \mu 1$ reaction mix containing the translated protein were used in further applications.

\subsubsection{Histone peptide pull-down experiments}

Histone peptide pull-down experiments were performed based on Wysocka et al. [131]. $30 \mu \mathrm{l}$ 50:50 slurry of Pierce ${ }^{\circledR}$ Avidin Agarose Resin per pull-down were washed three times with PBS (1 min incubation in buffer followed by centrifugation with $500 \mathrm{xg}, 1 \mathrm{~min}$ ). Then, $10 \mu \mathrm{g}$ of histone peptide (2.1.6) per $30 \mu \mathrm{l}$ bead slurry were added and the samples were incubated for $3 \mathrm{hrs}$ while shaking $(1400 \mathrm{rpm})$ in a thermomixer comfort at RT. Unbound peptides were removed by three washing steps (PBS as before). Purified recombinant protein (2.3.5) or programmed TNT reaction mix (2.3.6) was added to the beads in $750 \mu$ PD150 buffer (20 mM HEPES, $150 \mathrm{mM}$ $\mathrm{KCl}, 0.2 \%$ Triton $^{\circledR} \mathrm{X}-100,20 \%$ glycerol, $\mathrm{pH}$ 7.9).

For pull-down experiments using purified recombinant wild-type or mutant LIN-61 proteins, the PD150 buffer was supplemented with $0.5 \% \mathrm{w} / \mathrm{v}$ low-fat dry milk (blocking solution (milk)) or with 25\% C. elegans extract (blocking solution (C. elegans extract)) when indicated.

Samples were incubated $\mathrm{ON}$ at $4^{\circ} \mathrm{C}$ under constant rotation and then washed six times with $1 \mathrm{ml} \mathrm{PD} 150$ buffer each for $5 \mathrm{~min}$ at $4^{\circ} \mathrm{C}$ (centrifugation $500 \mathrm{xg}, 1 \mathrm{~min}$ ). Proteins 
recovered on the peptide-coated beads were eluted by boiling in $40 \mu 1$ protein sample buffer. After SDS-PAGE separation, western blotting was performed $(2.3 .1,2.3 .2)$.

\subsection{Generation of LIN-61 specific antibodies}

\subsubsection{Generation of $\alpha L I N-61$ peptide antibodies}

Charles River Laboratory raised antisera in guinea pigs against LIN-61 sequences in collaboration with the Peptide Sceciality Laboratories (PSL) GmbH by injecting each of the following peptides (produced by Dr. Rackwitz, PSL GmbH).

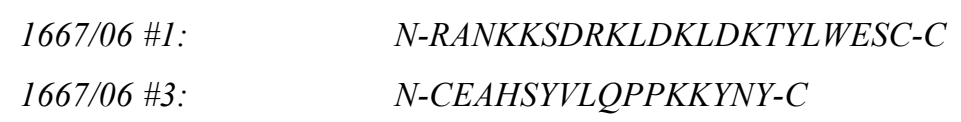

The sera obtained were affinity purified using corresponding prepackaged peptide columns (obtained from the PSL GmbH). Briefly, $4 \mathrm{ml}$ of guinea pig serum was diluted in an equal volume of PBS and incubated with $0.5 \mathrm{ml}$ peptide-bound column matrix $\mathrm{ON}$ at $4^{\circ} \mathrm{C}$ while slowly rotating. The suspension was filled back into the column, and the serum/PBS mixture was allowed to pass the column twice by gravity flow. Afterwards, the column was washed first with $30 \mathrm{ml}$ PBS and then with $20 \mathrm{ml}$ $10 \mathrm{mM}$ Na-phosphate $\mathrm{pH}$ 6.8. To elute peptide specific antibodies, ten times $0.5 \mathrm{ml}$ $0.1 \mathrm{M}$ glycine $\mathrm{pH} 2.4$ was added, and when added each flow-through fraction was collected in a tube loaded with $35 \mu \mathrm{l} 2 \mathrm{M} \mathrm{K}_{2} \mathrm{HPO}_{4}$ for $\mathrm{pH}$ neutralization. Fractions containing the highest protein concentration of eluted antibodies were determined using the Micro BCA Protein Assay Kit (Thermo Fisher Scientific), according to manufacturer's protocol, and pooled. Purified antibodies were aliquoted and stored at $-80^{\circ} \mathrm{C}$. Specificity of antibodies against LIN-61 was tested on crude C. elegans lysate (2.5.8) by western blotting (2.3.2). Purified antibodies against peptide 1667/06\#1 showed specificity in western blot analysis (see Figure 2-2) and were used for experiments in the present study (named $\alpha \mathrm{LIN}-61$ (guinea pig)).

\subsubsection{Generation of antibodies using recombinant LIN-61 for immunization}

Recombinant non-tagged LIN-61 was expressed and purified under denaturing conditions by Dr. Wolfgang Fischle. The expression construct used was the lin-61 coding sequence cloned into pET11a (2.2.8). 
By immunizing two rabbits with the purified protein, the company Charles River Laboratory raised antisera according to their proprietary protocol.

Specificity of antibodies against LIN-61 was tested on crude C. elegans lysate (2.5.8) by western blotting (2.3.2). Antibodies that showed specificity in western blot analysis (Figure 2-2) were used for experiments in the present study (named $\alpha$ LIN-61 (rabbit)).
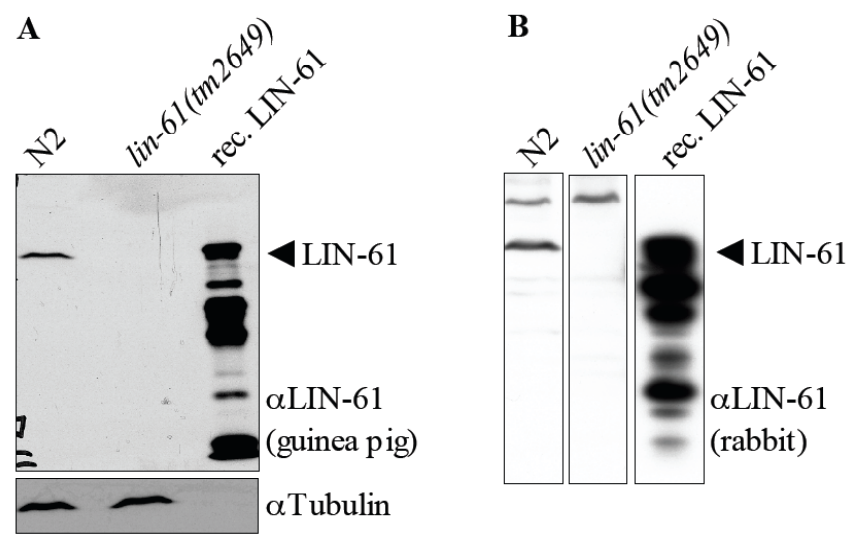

Figure 2-2: Western Blot analysis of generated $\alpha \mathrm{LIN}-61$ antibodies

Crude C. elegans lysates generated from $\mathrm{N} 2$ wild-type and from lin61(tm2649) mutant worms (Figure 3-9) and recombinant purified LIN61 were separated by SDS PAGE and analyzed using (A) $\alpha$ LIN-61 (guinea pig) antibodies (2.4.1) in a 1:1000 dilution ( $\alpha$ Tubulin was used as loading control) and (B) $\alpha$ LIN-61 (rabbit) antibodies (2.4.2) in a 1:4000 dilution. Arrowhead indicate LIN-61

\subsection{C. elegans based methods}

\subsubsection{Examination of C. elegans}

For handling and observation C. elegans, cultures or individual worms were monitored with a dissecting microscope illuminated from below while placed on a NGM plate (2.5.2) or an agarose pad. To generate agarose pads, $2 \%$ of agarose was boiled in $\mathrm{H}_{2} \mathrm{O}$ before a drop of this solution was spotted onto a coverslip and made even using a second coverslip. For analysis of $C$. elegans at $24.5^{\circ} \mathrm{C}, \mathrm{F} 1$ offspring was analyzed of hermaphrodites that were shifted at the adult stage from $20^{\circ} \mathrm{C}$ to $24.5^{\circ} \mathrm{C}$ for one night to lay eggs.

To look at individual worms in more detail, single animals were picked into a drop of $2 \% \mathrm{v} / \mathrm{v} \mathrm{NaN}_{3}$ on an agarose pad and examined under the inverted research microscope ECLIPSE Ti-E with DIC optics. Images of the examined worms were taken on a digital camera.

\subsubsection{Culturing C. elegans on plates}

C. elegans strains were cultured as described in [124] and [132] on Nematode Growth Medium (NGM) plates $(0,3 \% \mathrm{w} / \mathrm{v} \mathrm{NaCl}, 0,25 \% \mathrm{w} / \mathrm{v}$ peptone, $1.7 \% \mathrm{w} / \mathrm{v}$ agar, $5 \mathrm{mg} / \mathrm{l}$ 
cholesterol, $1 \mathrm{mM} \mathrm{CaCl}, 1 \mathrm{mM} \mathrm{MgSO}_{4}, 25 \mathrm{mM}$ K-phosphate buffer $\mathrm{pH}$ 6.0, 100 $\mu \mathrm{g} / \mathrm{ml}$ streptomycin, $10 \mu \mathrm{g} / \mathrm{ml}$ nystatin) that were seeded with an E. coli OP50-1 lawn at either $15{ }^{\circ} \mathrm{C}, 20{ }^{\circ} \mathrm{C}$ or $24.5{ }^{\circ} \mathrm{C}$. To generate larger quantities of worms growing on one agar plate, Super NGM plates $(0,3 \% \mathrm{w} / \mathrm{v} \mathrm{NaCl}, 2 \% \mathrm{w} / \mathrm{v}$ peptone, $1.7 \% \mathrm{w} / \mathrm{v}$ agar, $15 \mathrm{mg} / \mathrm{l}$ cholesterol, $1 \mathrm{mM} \mathrm{CaCl}_{2}, 1 \mathrm{mM} \mathrm{MgSO}_{4}, 25$ mM K-phosphate buffer pH 6.0, $100 \mu \mathrm{g} / \mathrm{ml}$ streptomycin, $10 \mu \mathrm{g} / \mathrm{ml}$ nystatin) were used. To transfer C. elegans from one plate to another, a spatula was used to move a chunk of agar from an old plate to a new plate. Alternatively, single worms were picked for transfer with a platinum wire that was mounted to a pasteur pipette. To harvest worms for further experiments, worms were washed off the plates with water into a test tube and allowed to settle down on ice. After washing the worm pellet a few times with water, the worms were either directly used or flash frozen in liquid $\mathrm{N}_{2}$ and stored at $-80^{\circ} \mathrm{C}$.

\subsubsection{Culturing C. elegans in liquid medium}

Large quantities of $C$. elegans were obtained by growing them in liquid medium as described in [133]. As starting material 10 NGM plates (2.5.2) overgrown with starved L1/L2 larvae were harvested by washing them off the plates with water into a $50 \mathrm{ml}$ tube. The worms were allowed to settle down on ice and washed with water several times. The washed worm pellet was used to inoculate a 2.51 flask containing $500 \mathrm{ml} \mathrm{S}$ Medium (0.1 M NaCl, $50 \mathrm{mM} \mathrm{K-phosphate} \mathrm{buffer} \mathrm{pH} 6.0,5 \mathrm{mg} / 1 \mathrm{w} / \mathrm{v}$ cholesterol, $10 \mathrm{mM} \mathrm{K-Citrate} \mathrm{pH} \mathrm{6.0,} 6.4 \mathrm{mM}$ EDTA, $2.5 \mathrm{mM} \mathrm{FeSO}_{4}, 1 \mathrm{mM} \mathrm{MnCl}$, $1 \mathrm{mM} \mathrm{ZnSO}_{4}, 0.1 \mathrm{mM} \mathrm{CuSO}_{4}, 3 \mathrm{mM} \mathrm{CaCl}_{2}, 3 \mathrm{mM} \mathrm{MgSO}_{4}$ ) that was supplemented with $5 \mathrm{ml}$ concentrated $\mathrm{HB} 101$ bacteria solution. The flask was incubated at $20^{\circ} \mathrm{C}$ and shaken at $120 \mathrm{rpm}$. The growth of the worms was carefully followed by transferring small aliquots of worm suspension onto a glass slide and inspecting them with a dissecting microscope. Additional bacteria solution was added as needed. The unsynchronized culture was harvested after three to five days when the majority of worms were adults. To harvest the culture, it was decanted into $50 \mathrm{ml}$ tubes and the worms were either allowed to settle down on ice or centrifuged for 2 min at 1500xg and $4{ }^{\circ} \mathrm{C}$. The worm pellet was washed several times, flash frozen in liquid $\mathrm{N}_{2}$ and stored at $-80^{\circ} \mathrm{C}$. 


\subsubsection{Synchronization of C. elegans}

To synchronize $C$. elegans, a population of worms with a high percentage of hermaphrodites containing eggs were washed several times with $\mathrm{H}_{2} \mathrm{O}$ and then treated with bleach-solution $(0.5 \mathrm{M} \mathrm{NaOH}, 1.2 \% \mathrm{NaClO})$ in a test tube. The tube was incubated for $10 \mathrm{~min}$ (with frequent vortexing in between) until the worms were resolved. Centrifugation for two min at 1500xg pelleted the released eggs. The egg pellet was washed three times and then incubated in M9-buffer $\left(0.3 \% \mathrm{w} / \mathrm{v} \mathrm{KH}_{2} \mathrm{PO}_{4}\right.$, $\left.0.6 \% \mathrm{w} / \mathrm{v} \mathrm{Na}_{2} \mathrm{HPO}_{4}, 0.5 \% \mathrm{w} / \mathrm{v} \mathrm{NaCl}, 1 \mathrm{mM} \mathrm{MgSO} 4\right) \mathrm{ON}$ at $20{ }^{\circ} \mathrm{C}$. The next day hatched L1 larvae were seeded onto fresh NGM plates.

In addition, this method was used to clean contaminated C. elegans stocks as bacterial contaminants and yeast are killed by this procedure [132].

\subsubsection{Freezing and recovery of C. elegans stocks}

To keep C. elegans stocks indefinitely, they were frozen and stored at $-80{ }^{\circ} \mathrm{C}$. As freshly starved L1-L2 stage larvae survive the freezing procedure best, several plates of worms that were depleted of food were washed off with water, and the worms were collected in a tube. The worms were allowed to settle down on ice, and the water was removed to a residual volume of three to five milliliters. An equal volume of freezing solution $\left(0.3 \% \mathrm{w} / \mathrm{v} \mathrm{KH}_{2} \mathrm{PO}_{4}, 0.6 \% \mathrm{w} / \mathrm{v} \mathrm{Na} \mathrm{HPO}_{4}, 0.5 \% \mathrm{w} / \mathrm{v} \mathrm{NaCl}, 1 \mathrm{mM} \mathrm{MgSO}\right.$, $30 \% \mathrm{v} / \mathrm{v}$ glycerol) was added. After mixing, aliquots of $1 \mathrm{ml}$ in cryotubes were packed in a styrofoam box with slots for holding the tubes, to ensure a slow cooling of the samples, and placed in a $-80{ }^{\circ} \mathrm{C}$ freezer [124], [132].

To recover $C$. elegans stocks, a frozen aliquot of the stock was thawed at RT until melted and poured onto a bacteria seeded NGM plate.

\subsubsection{Single worm PCR}

Single worm PCR is a method to analyze individual C. elegans for the presence of particular deletions in genes of interest. A single hermaphrodite was picked into a PCR tube containing $2.5 \mu \mathrm{l}$ single worm PCR lysis buffer $(10 \mathrm{mM}$ Tris, $50 \mathrm{mM} \mathrm{KCl}$, $2.5 \mathrm{mM} \mathrm{MgCl} 2,0.45 \% \mathrm{NP}-40,0.45 \%$ Tween $20,0.01 \%$ gelatin, $100 \mu \mathrm{g} / \mathrm{ml}$ Proteinase-K, $\mathrm{pH} 8.3$ ) and frozen at $-80^{\circ} \mathrm{C}$ for $30 \mathrm{~min}$ to freeze-crack the worm. The sample was incubated at $60^{\circ} \mathrm{C}$ for $60 \mathrm{~min}$ to degrade the worm proteins by proteinase $\mathrm{K}$ before the enzyme was inactivated at $95^{\circ} \mathrm{C}$ for $15 \mathrm{~min}$. Then, a PCR was performed 
using three primers that can amplify a specific genomic DNA fragment. One forward and one reverse primer were located outside and one reverse primer was located within a particular deletion mutation. In case of wild-type and homozygous deletion mutant worms, one genomic DNA fragment of a particular size was made due to a short elongation time. In the case of heterozygous worms, two DNA fragments corresponding to the wild-type and mutant deletion allele were obtained. As the two amplified DNA fragments differ in size, this method allows to detect whether a worm is heterozygous or homozygous for the particular deletion mutation or whether it is wild-type for the gene of interest (2.2.4). The following PCR protocol was carried out: $22.5 \mu \mathrm{l}$ single worm PCR reaction buffer $\left(2.2 \mathrm{mM} \mathrm{MgCl}_{2}, 222 \mu \mathrm{M}\right.$ of each $\mathrm{dNTP}, 0.7 \mu \mathrm{M}$ of each chosen primer, 0.5 unit/22.5 $\mu 1$ Taq polymerase (Fermentas) in $1 \mathrm{x}$ reaction buffer provided by the manufacturer) was added to the sample and the PCR was performed using the sw-PCR protocol $\left(1 \mathrm{~min}\right.$ at $94^{\circ} \mathrm{C}-\left[30 \mathrm{sec}\right.$ at $94^{\circ} \mathrm{C}-30$ sec at $57^{\circ} \mathrm{C}-40 \mathrm{sec}$ at $\left.72^{\circ} \mathrm{C}\right]_{40 \mathrm{x}}-5 \mathrm{~min}$ at $72^{\circ} \mathrm{C}$ ). When more than one single worm PCR had to be performed, the worm was picked into $5 \mu$ (for two PCRs) or $7.5 \mu 1$ (for three PCRs) of single worm PCR lysis buffer. The sample was then separated into $2.5 \mu 1$ fractions for the different PCR reactions.

The following primers were used to analyze hermaphrodites for the lin-61 allele lin61( $\operatorname{tm} 2649)$ :

lin sw for2id gr: $\quad$ '-GACAACGTCTTGAACTATTAAATTATTCC-3'

lin sw R1: 5'-GTGTGCTCAATATATTCCTTTTTCG-3'

lin-delmut-for: 5'-CGGACAGAAAACTCGATAAGACCTAC-3'

To analyze hermaphrodites for the $m b t r-1$ allele $m b t r-1(n 4775)$, the single worm PCR was performed with some modifications. The single worm PCR reaction buffer was supplemented with 4\% DMSO, and two separate PCR reactions were conducted. For PCR reaction one, a primer pair was included that amplifies only the mbtr- 1 wild-type allele. For PCR reaction two, a primer pair was included that amplifies a DNA fragment only present in the mbtr-1 mutant allele mbtr-1(n4775). The following primers were used:

$\begin{array}{lll}\text { PCR reaction 1: } & Y 48 \text { sw for del: } & \text { 5'-GGAGAGAAGGCACATGTTTGAGG-3' } \\ & \text { Y48 sw rev: } & \text { 5'-GACTTTATTTTTCGTATTATTTTCTTCATTTC-3' } \\ \text { PCR reaction 2: } & \text { y48 sw for 1: } & \text { 5'-CTATTCTATCGTCGAAGAAATCCAGTTG-3' } \\ & Y 48 \text { sw rev: } & \text { see above }\end{array}$

To analyze hermaphrodites for the lin-15A allele lin-15A(n767), a modified single worm PCR reaction was performed, as only two primers were used: 


$\begin{array}{ll}\text { lin15a sw for: } & \text { 5'-TGGCCATGATATCTATAGAGCACTG-3' } \\ \text { lin15a-sw rev: } & \text { 5'-GATTATCTTTCCCATTGATGAGTTG-3' }\end{array}$

The following primers were used to analyze hermaphrodites for the met-2 allele met2(n4256):

met-2 sw for: 5'-GGATTGAATTCGAAAAATGGACCG-3'

met-2 sw rev: $\quad 5$ '-CTGAGCAGCATTCATCTTCGC-3'

met-2 swidel for: $\quad$ '-GCAGAGCTCATAGATCCGATTTTC-3'

The following primers were used to analyze hermaphrodites for the $h p l-2$ allele $h p l$ 2(tm1489):

sw-hpl2 for1: $\quad$ '-CTTCTGGCTGTGTCGTTTGCG-3'

sw-hpl2rev: $\quad$ ''-CTGTACGGTGATTGTCTGTGAG-3'

sw-hpl2for2indel: $\quad 5$ '-GCTCGCTTTGTTGATTTGCTCG-3'

\subsubsection{C. elegans crosses}

Crosses were set up to generate double mutant $C$. elegans strains. In a first step, the frequency of males had to be enhanced for one strain, as the occurrence of males (that arise by spontaneous $\mathrm{X}$ chromosome nondysjunction) is very low in wild-type populations $(\sim 0.1 \%)$. To do so, several plates were searched for males that were then picked to a new NGM plate together with a few hermaphrodites. This plate could be kept as a male stock plate since, in contrast to progeny that result from selfing, the F1 progeny that results from such mating consist of $\sim 50 \%$ males. To start a cross, $10-20$ males of one strain were put together with 1-4 L4 hermaphrodites of the other strain and incubated for two days at $20{ }^{\circ} \mathrm{C}$. If a male mates with a hermaphrodite, the majority of the progeny will be cross-progeny, because male sperm out competes hermaphrodite sperm. The hermaphrodites were singled to new NGM plates to lay eggs. If the offspring consists of $\sim 50 \%$ males, the crossing was successful. F1 L4 hermaphrodites were singled again and allowed to lay eggs. After laying eggs, the hermaphrodites were picked and analyzed by single worm PCR (2.5.6) to test, if they had been heterozygous for the particular deletion mutations. Progeny of heterozygous hermaphrodites were singled and analyzed by single worm PCR to test if they were homozygous for the particular deletion mutations. The siblings of a homozygous hermaphrodite were the origin of a new double mutant strain. The following C. elegans double mutant strains were generated by crossing:

mbtr-1 lin-61: $\quad$ mbtr-1(n4775) lin-61(tm2649) I

lin-61; lin-15A: lin-61(tm2649)I; lin-15A(n767)X 
lin-61; met-2: $\quad$ lin-61(tm2649)I; met-2(n4256)III

lin-61; hpl-2: $\quad$ lin-61(tm2649)I; hpl-2(tm1489)III

mbtr-1; hpl-2: $\quad$ mbtr-1(n4775)I; hpl-2(tm1489)III

In addition, crosses were set up to eliminate background mutations in single mutant $C$. elegans strains by crossing single mutant worms with wild-type N2. By this method, lin-61(tm2649) was backcrossed five times to N2 and $m b t r-1(n 4775)$ was backcrossed three times to $\mathrm{N} 2$.

\subsubsection{C. elegans protein extract preparation}

C. elegans protein extract was either prepared according to Cheeseman et al. [134] with some modifications or to Wysocka et al. [131] with some modifications.

For both extract preparations, $5 \mathrm{~g}$ of frozen C. elegans, derived from liquid C. elegans cultures (2.5.3), were grounded using a pre-chilled mortar and pestle in liquid $\mathrm{N}_{2}$.

\subsubsection{Protein extract preparation based on Cheeseman et al. (2004)}

The grounded worm pellet (see above) was supplemented with an equal volume of $2 \mathrm{x}$ Cheeseman buffer (50 mM HEPES, 2 mM EGTA, 2 mM MgCl $2,200 \mathrm{mM} \mathrm{KCl,} \mathrm{20 \%}$ glycerol, 0.1\% NP-40, 2x Roche's EDTA-free Complete Protease Inhibitor Cocktail, $\mathrm{pH}$ 7.4) while grounding and then thawed on ice. The grounded worms were sonicated with a Branson Digital Sonifier using the following settings: $30 \%$ amplitude for 3 min total ( $15 \mathrm{~s} \mathrm{on;} 59 \mathrm{~s}$ off), 40\% amplitude for $30 \mathrm{~s}$ (15 s on; $59 \mathrm{~s}$ off). Extract was clarified by centrifugation at $50000 \mathrm{xg}$ for $60 \mathrm{~min}$ at $4^{\circ} \mathrm{C}$ and the supernatant was transferred to a new tube. The protein concentration of the extract was determined using the Coomassie Plus (Bradford) Protein Assay (Thermo Scientific), according to the manufacturer's protocol.

\subsubsection{Protein extract preparation based on Wysocka et al. (2006)}

The grounded worm pellet (see above) was supplemented with an equal volume of $2 \mathrm{x}$ buffer A (10 mM HEPES, $30 \mathrm{mM} \mathrm{MgCl}$, $20 \mathrm{mM} \mathrm{KCl,} 1 \mathrm{mM}$ DTT, 1x Roche's EDTA-free Complete Protease Inhibitor Cocktail, $\mathrm{pH}$ 7.9) while grounding before they were thawed. The grounded worm/buffer suspension was sonicated as described in the previous section and then centrifuged for $10 \mathrm{~min}$ at $18000 \mathrm{xg}$ at $4^{\circ} \mathrm{C}$. The pellet was resuspended in buffer C (20 mM HEPES, $420 \mathrm{mM} \mathrm{KCl}, 1.5 \mathrm{mM} \mathrm{MgCl} 2,0.2 \mathrm{mM}$ EDTA, 25\% v/v glycerol, 1x Roche's EDTA-free Complete Protease Inhibitor 
Cocktail, $\mathrm{pH}$ 7.4) and incubated for $2 \mathrm{hrs}$ at $4^{\circ} \mathrm{C}$ while shaking at high setting. Samples were centrifuged for $10 \min \left(18000 \mathrm{xg}, 4^{\circ} \mathrm{C}\right)$ and the supernatant, containing the soluble nuclear protein extract, was kept. To lower the salt concentration, extract was dialyzed against buffer D (20 mM HEPES, $150 \mathrm{mM} \mathrm{KCL}, 0.2 \mathrm{mM}$ EDTA, 0.2\% v/v Triton-X100, 20\% v/v glycerol, $1 \mathrm{mM}$ DTT, $2 \mathrm{mM}$ PMSF, $2 \mathrm{mM}$ benzamidine, $\mathrm{pH}$ 7.9). The protein concentration of the extract was determined as described above.

\subsubsection{Generation of crude $C$. elegans lysate for western blot analysis}

A minimum of 50 animals were transferred to a $1.5 \mathrm{ml}$ tube and washed with $\mathrm{H}_{2} \mathrm{O}$. For homogenization, an equal amount $(\mathrm{w} / \mathrm{v})$ of nematode solubilization buffer $(0.3 \%$ (v/v) ethanolamine, $2 \mathrm{mM}$ EDTA, $1 \mathrm{mM}$ PMSF, $5 \mathrm{mM}$ DTT) [135] was added. The sample was sonicated for $20 \mathrm{~min}$ in $30 \mathrm{~s}$ on; $30 \mathrm{~s}$ off intervals in the Bioruptor ${ }^{\mathrm{TM}}$. Afterwards an equal volume of $2 x$ protein sample buffer was added and the sample was boiled for $5 \mathrm{~min}$. After clarification (centrifugation for $10 \mathrm{~min}$ at $16000 \mathrm{xg}$ at $4^{\circ} \mathrm{C}$ ), 15-20 $\mu 1$ of the supernatant was loaded onto a SDS PAGE gel (2.3.1), which was processed for western blotting (2.3.2).

\subsubsection{Histone peptide pull-down experiments using C. elegans extract}

Histone peptide pull-down experiments were conducted exactly as described in (2.3.7) but $\sim 1 \mathrm{ml} \mathrm{C.} \mathrm{elegans} \mathrm{extract} \mathrm{was} \mathrm{used} \mathrm{instead} \mathrm{of} \mathrm{recombinant} \mathrm{protein.}$

\subsubsection{Gel filtration of C. elegans extract}

Gel filtration chromatography was performed using either $300 \mu 1$ of N2 C. elegans protein extract that was generated based on the Cheeseman et al. protocol (protein concentration: $\sim 10 \mathrm{mg} / \mathrm{ml}$ ) or $300 \mu \mathrm{l}$ of $\mathrm{N} 2$ C. elegans protein extract that was generated based on the Wysocka et al. protocol (protein concentration: $\sim 1.2 \mathrm{mg} / \mathrm{ml}$ (2.5.8). Protein extract was applied to a Superose 6 30/100 GL column (GE Healthcare) by using an Äkta Explorer FPLC instrument with the following buffer: 25 mM HEPES, $1 \mathrm{mM}$ EGTA, $1 \mathrm{mM} \mathrm{MgCl} 2,100 \mathrm{mM} \mathrm{KCl,} \mathrm{5 \%} \mathrm{glycerol,} \mathrm{0.05 \%} \mathrm{NP-40,}$ 1x Roche's EDTA-free Complete Protease Inhibitor Cocktail, pH 7.4. For elution, the same buffer was used and fractions of $500 \mu 1$ were collected.

To monitor the elution profile of LIN-61, fractions were precipitated by trichloroacetic acid (TCA)-precipitation. $20 \mu \mathrm{l}$ of $100 \%$ ice-cold TCA was added to each $500 \mu \mathrm{l}$ fraction and incubated for $15 \mathrm{~min}$ on ice. After centrifugation (16000xg, 
$10 \mathrm{~min}, 4^{\circ} \mathrm{C}$ ), the pellet was washed two times with $1 \mathrm{ml}$ ice-cold acetone. The pellet was air dried and resuspended by boiling in protein sample buffer. Samples were analyzed by SDS PAGE and western blotting (2.3.2).

\subsubsection{Immunprecipitation of C. elegans extract}

\subsubsection{1 $\alpha$ LIN-61 immunprecipitation of C. elegans extract}

Immunoprecipitation experiments using $\alpha$ LIN-61 (rabbit) antibodies (2.4.2) were performed using $C$. elegans protein extracts that were generated based on the Cheeseman et al. protocol (2.5.8). $\alpha$ LIN-61 (rabbit) antibodies were crosslinked to Dynabeads ${ }^{\circledR}$ M-280 Sheep anti Rabbit IgG (Invitrogen), according to manufacturer's protocol. Briefly, $30 \mu \mathrm{l}$ of $\alpha \mathrm{LIN}-61$ (rabbit) antibodies were incubated with $60 \mu \mathrm{l}$ beads slurry $\mathrm{ON}$ at $4{ }^{\circ} \mathrm{C}$ under constant rotation. Unbound antibodies were removed by washing the beads three times in $1 \mathrm{ml}$ PBS before the beads were resuspended in $0.2 \mathrm{M}$ triethanolamine $\mathrm{pH}$ 8.2. Afterwards, the beads were incubated for $60 \mathrm{~min}$ in 1 $\mathrm{ml}$ of $20 \mathrm{mM}$ DMP in $0.2 \mathrm{M}$ triethanolamine $\mathrm{pH} 8.2$ at RT while rotating. To quench the crosslinking, the buffer was exchanged to $1 \mathrm{ml} 50 \mathrm{mM}$ Tris $\mathrm{pH} 7.5$ and incubated for $60 \mathrm{~min}$ at RT. The beads were washed three times with PBS before the bead suspension volume was adjusted to the initial volume with PBS.

For immunoprecipitation, the prepared bead slurry was incubated with $8 \mathrm{mg}$ protein extract. The suspension was incubated $\mathrm{ON}$ at $4^{\circ} \mathrm{C}$ under constant rotation. To remove unbound proteins, the beads were washed either three times with 1x Cheeseman buffer or four times with high salt buffer (25 mM HEPES, $1 \mathrm{mM}$ EGTA, $1 \mathrm{mM}$ $\mathrm{MgCl}_{2}, 300 \mathrm{mM} \mathrm{KCl}, 20 \%$ glycerol, 0.5\% NP-40, 2x Roche's EDTA-free Complete Protease Inhibitor Cocktail, $\mathrm{pH}$ 7.4). The bound protein fraction was eluted from the beads by boiling in $40 \mu 1$ protein sample buffer.

\subsubsection{2 $\alpha$ GFP immunprecipitation of $C$. elegans extract}

Immunoprecipitation experiments with $\alpha$ GFP antibody were conducted using $C$. elegans protein extracts that were generated based on the Cheeseman et al. protocol (2.5.8). $5 \mu \mathrm{l} \alpha \mathrm{GFP}$ antibody were bound to $40 \mu \mathrm{l}$ Dynabeads ${ }^{\circledR}$ M-280 Sheep anti mouse $\mathrm{IgG}$ by incubation for $2 \mathrm{hrs}$ at $4^{\circ} \mathrm{C}$ under constant rotation. Unbound antibody was removed by washing the beads 3 times in $1 \mathrm{ml}$ PBS. Then, $10 \mu 1$ of $10 \% \mathrm{w} / \mathrm{v}$ BSA and $6 \mathrm{mg}$ protein extract was added to the beads, and the suspension was 
incubated $\mathrm{ON}$ at $4^{\circ} \mathrm{C}$ under constant rotation. To remove unbound proteins, the beads were washed four times with 1x Cheeseman buffer before the bound protein fraction was eluted from the beads by boiling in $40 \mu 1$ protein sample buffer.

\subsubsection{C. elegans RNA isolation and Reverse Transcription}

To isolate C. elegans RNA, $1 \mathrm{ml}$ TRIzol ${ }^{\circledR}$ Reagent and $10 \mu 1$ 2-mercaptoethanol were added to $0.2 \mathrm{~g}$ frozen worm pellet. The sample was thawed while intensively shaken and afterwards frozen in liquid $\mathrm{N}_{2}$. This procedure was repeated three times. The sample was incubated at RT for $5 \mathrm{~min}$ and then centrifuged $(16000 \mathrm{xg}$ for $10 \mathrm{~min}$ at $4^{\circ} \mathrm{C}$ ) to remove insoluble material. The supernatant was split into Phase Lock Gel ${ }^{\mathrm{TM}}$ ( $2 \mathrm{ml}$, heavy) tubes (600 $\mu \mathrm{l}$ each) and supplemented with $120 \mu \mathrm{l}$ chloroform/isoamyl alcohol $(49: 1 \mathrm{v} / \mathrm{v})$. The samples were vigorously shaken and incubated at RT for 3 min before centrifugation $\left(12000 \mathrm{xg}\right.$ for $15 \mathrm{~min}$ at $4^{\circ} \mathrm{C}$ ). The upper aqueous phases were combined in a new tube, mixed with $500 \mu 1$ isopropanol per $1 \mathrm{ml} \mathrm{TRIzol}^{\circledR}$ Reagent (initial volume) and incubated for $10 \mathrm{~min}$ at RT to precipitate the isolated RNA. The precipitate was collected by centrifugation $\left(16000 \mathrm{xg}, 30 \mathrm{~min}, 4^{\circ} \mathrm{C}\right)$ and washed with $75 \%$ ethanol before drying for $5-10 \mathrm{~min}$ at $37^{\circ} \mathrm{C}$. Afterwards, the RNA was dissolved in $\mathrm{H}_{2} \mathrm{O}$.

To deplete residual DNA, $3 \mu \mathrm{g}$ RNA was incubated with $0.8 \mu 1$ 10x DNaseI buffer (100 mM Tris, $25 \mathrm{mM} \mathrm{MgCl}_{2}, 5 \mathrm{mM} \mathrm{CaCl}_{2}, \mathrm{pH}$ 7.6), $1 \mu \mathrm{l}$ RNase-out and $0.8 \mu \mathrm{l}$ DNaseI in a $8 \mu \mathrm{l}$ reaction volume for $30 \mathrm{~min}$ at $37^{\circ} \mathrm{C}$. The reaction was stopped by adding $0.4 \mu 10.1 \mathrm{M}$ EDTA followed by incubation for $10 \mathrm{~min}$ at $75^{\circ} \mathrm{C}$.

$8 \mu \mathrm{l}$ of DNaseI digested RNA solution was used to synthesize first-strand cDNA using the SuperScript ${ }^{\text {tm }}$ III First-Strand Synthesis System for RT-PCR (Invitrogen). The synthesis was done in a volume of $20 \mu \mathrm{l}$ according to the manufacturer's protocol using the provided Oligo(dT) primers.

\subsubsection{Quantitative PCR analysis of lin-3 transcripts}

The relative abundance of lin-3 mRNA in wild-type and mutant C. elegans strains was measured by quantitative real-time PCR using $\mathrm{SYBR}^{\circledR}$ green [89].

As template, synthesized cDNA (2.5.12) derived from RNA (2.5.12) that was isolated from synchronized wild-type and mutant $C$. elegans larvae grown at $20^{\circ} \mathrm{C}$ and harvested at late L2 to late L3 stages was used. The following primers were used to 
amplify a $236 \mathrm{bp}$ fragment that spans the exon 6 - exon 7 junction of the reverse transcribed lin-3 mRNA:
lin-3 67 for:
5'-GCGTTCGCATTTCTCATTG-3'
lin-3 67 rev:
5'-GTTGATGATATATTTGGAGGTA-3'

As internal control, a 203 bp fragment of rpl-26 mRNA spanning the exon 1 - exon 2 junction was amplified with the following primers:
rpl-26 12 for:
5'-CTCACCAAGGAGCTCCGCACC-3'
rpl-26 12 rev:
5'-GAAGGGTGGATTCCGATGTGC-3'

The quantitative real-time PCR reactions were done in triplicate and were performed by assembling a reaction mix of $0.125 \mu 1$ of a $20 \mu \mathrm{l}$ cDNA synthesis reaction (corresponding to $\sim 19 \mathrm{ng}$ total RNA), $1.25 \mu \mathrm{M}$ of each primer and $10 \mu \mathrm{l}$ of $2 \mathrm{x}$ iQ $\mathrm{SYBR}^{\circledR}$ Green Supermix in a $20 \mu$ reaction volume that was processed in a DNA Engine OPTICON real-time PCR machine with the following program: $15 \mathrm{~min}$ at $95^{\circ} \mathrm{C}-\left[15 \mathrm{sec} \text { at } 94^{\circ} \mathrm{C}-55 \mathrm{sec} \text { at } 55^{\circ} \mathrm{C}-30 \mathrm{sec} \text { at } 72^{\circ} \mathrm{C} \text { - read plate }\right]_{40 \mathrm{x}}$ - perform melting curve from $60^{\circ} \mathrm{C}$ to $95^{\circ} \mathrm{C}$, read every $0.3^{\circ} \mathrm{C}$.

To calculate the relative gene expression level of lin-3 in mutant C. elegans with respect to wild-type, the $2^{(-\Delta \Delta \mathrm{Ct})}$ method [136] was used with

$\Delta \Delta \mathrm{Ct}_{\text {lin-3 amplicon of mutant strain }}=\left(\mathrm{Ct}_{\text {lin-3 amplicon of mutant strain }}-\mathrm{Ct}_{\mathrm{rpl}-26}\right.$ amplicon of mutant strain $)-$ $\left(\mathrm{Ct}_{\text {lin-3 }}\right.$ amplicon of wild-type $-\mathrm{Ct}_{\mathrm{rpl}-26}$ amplicon of wild-type). For analysis, the data of two independent RNA isolations from independent worm growths were combined and the STDEV was calculated from the resulting six $2^{(-\Delta \Delta \mathrm{Ct})}$ values.

\subsubsection{RNAi feeding experiments}

RNAi feeding experiments were performed to score the loss-of-function phenotype of lin-15A in wild-type and different $C$. elegans mutant strains. The experimental procedure was according to the protocol in Kamath et al. [137] with some minor modifications. Briefly, $5 \mathrm{ml}$ LB-Amp media was inoculated with a fresh colony of Ht115(DE3) bacteria ZK678.1 (containing the lin-15A cDNA cloned into p14440Dest) and shaken for $8 \mathrm{hrs}$ at $37^{\circ} \mathrm{C} .100 \mu \mathrm{l}$ of the bacterial culture was streaked onto each NGM feeding plate (NGM plate including $100 \mu \mathrm{g} / \mathrm{ml}$ ampicilin, $12.5 \mu \mathrm{g} / \mathrm{ml}$ tetracyclin, $1 \mathrm{mM}$ IPTG but no streptomycin). The plates were incubated $\mathrm{ON}$ to allow the bacteria to grow and to induce RNA expression. A drop of L1 larvae suspension ( 20 worms) derived from $C$. elegans wild-type and mutant strains that were bleached and allowed to hatch in M9 buffer (2.5.4) was spotted onto the plates. Plates 
were incubated at $24.5^{\circ} \mathrm{C}$ until the worms developed to early L4 stage ( $30 \mathrm{hrs}$ ). Three worms at a time were transferred to three new NGM feeding plates and allowed to lay eggs at $24.5^{\circ} \mathrm{C}$ before they were picked off the plates. The progeny of these worms (F1 generation) were further incubated at $24.5^{\circ} \mathrm{C}$ and scored for phenotypes. In parallel, the RNAi feeding experiment was performed using Ht115(DE3) bacteria containing the empty L4440 vector.

\subsubsection{C. elegans microinjection}

Microinjection of DNA mixtures into the distal gonad arms of C. elegans is a method to generate transgenic animals $[123,138]$. In general, the injected DNAs undergo homologous recombination with each other, which result in large extrachromosomal arrays that contain many copies of the injected DNAs in transgenic animals. Even though these repetitive arrays are usually unstable during cell division, they can become inheritable ranging from $\sim 10 \%$ to $\sim 90 \%$, with an average of about $50 \%$ inheritance in each generation $[123,138]$. To easily detect transgenic animals, the microinjected DNA mixture also contains a plasmid carrying a transformation marker like pRF4. pRF4 contains the mutant collagen rol-6 allele rol-6(su1006), and animals expressing it exhibit a helically twisted cuticle and move as a consequence in circles (roller phenotype) instead of forward or backward in a sinusoidal motion [139]. To generate transgenic C. elegans lines expressing either wild-type LIN-61 or a LIN-61 F452A W455A F459A triple point mutant protein in a lin-61; lin-15A (2.5.7) mutant background, microinjection was performed according to Mello \& Fire (1995) [140] with some modifications. To prepare the DNA solution for microinjection, a mix was assembled containing $80 \mathrm{ng} / \mathrm{ul} \mathrm{pRF} 4$ plasmid and $20 \mathrm{ng} / \mathrm{ul}$ of a purified SacII PspOMI restriction fragment $(2.2 .2,2.2 .4)$ of either the lin-61 (wild-type) gene or the lin-61 (triple aromatic cage point mutant) construct in $\mathrm{H}_{2} \mathrm{O}$. Individual young adult worms were transferred from a NGM plate into a drop of halocarbon oil that was pipetted onto an agarose pad (2.5.1). $2 \mu$ of the DNA mix were loaded into a Femotip ${ }^{\circledR}$ II microinjection needle connected to a pressurized injection system and controlled by a micromanipulator. DNA solution was injected into the gonads of individual young adult worms by pushing the needle into the worm gonad and applying pressure so that the solution flowed until the gonad noticeably swelled up. To recover microinjected worms, a drop of M9 buffer was added onto the worm to release it off the pad. After $5 \mathrm{~min}$, the worm was transferred to a new NGM plate. 
Surviving worms were incubated individually on plates until they laid eggs. F1 progeny displaying a roller phenotype were singled once more to separate possible individual transgenic lines that might inherit the extrachromosomal array. The plates were incubated for at least one week until the offspring were starved. Then, individuals with a roller phenotype were transferred to new plates. Transgenic lines were kept by continuous enrichment of worms with a roller phenotype. Seven lines with the genotype lin-61; lin-15A; Ex[lin-61(+) rol-6(su1006)] and four lines with the genotype lin-61; lin-15A; Ex[lin-61(triple aromatic cage point mutant) rol6(su1006)] were be established.

\subsubsection{C. elegans brood size assay}

To estimate the mean brood size of different C. elegans strains at $20^{\circ} \mathrm{C}$ or $24.5^{\circ} \mathrm{C}$, a brood size assay was performed [141]. Briefly, individual hermaphrodites at larvae stage (L3 - L4) were picked onto NGM plates and transferred to fresh NGM plates at successive days. Progeny were counted at larva stage. The mean brood size and standard deviation of a particular strain was calculated and corresponds to the average brood size of all assayed hermaphrodites that were not sterile. While counting the brood size, the percentage of sterile hermaphrodites of the particular strains was determined in parallel.

\subsubsection{C. elegans life span assay}

Life span assays were conducted to determine distinctions in life span (defined as the number of days an animal remains responsible to external stimuli) at $20^{\circ} \mathrm{C}$ and $24.5^{\circ} \mathrm{C}$ for wild-type and different mutant C. elegans strains [142]. Worms were grown on NGM plates until they reached the L4 stage and were then transferred (10 worms per plate) to NGM plates that were supplemented with $100 \mathrm{mM}$ FUDR and covered with $100 \mu 1$ of concentrated HB101 bacterial solution. FUDR was added to the plates to prevent growth of progeny without having an effect on the lifespan of C. elegans [143]. The life span of 50 animals per strain was determined by observing them every $1-3$ days. Worms were assessed as dead when they no longer responded to nudging with a platinum wire pick. The percentage of worms that were alive at each observation day was calculated. Worms that had crawled off the plates or could not be found were excluded from the calculation. The mean life $\left(m_{L S}\right)$ span of each strain, 
assessed from at least two independent experiments, was calculated from the number of worms that died each day using the following formula:

$$
\mathrm{m}_{\mathrm{LS}}=\frac{\mathrm{w}_{1}+\mathrm{w}_{2}+\ldots \mathrm{w}_{\mathrm{n}}}{\mathrm{n}}(\mathrm{w}=\text { number of worms that died at day } 1, \text { day } 2, \ldots \text { day } \mathrm{n})
$$

A two-tailed Student's t-test was used to calculate the significance of differences in the mean life span. 


\section{Results}

\subsection{Identification of $\mathrm{H} 3 \mathrm{~K} 9 \mathrm{me} 3$ binding proteins in C. elegans}

To investigate chromatin factors directly involved in the readout of the repressive chromatin mark H3K9me3 in C. elegans, I started by identifying effector proteins that bind to $\mathrm{H} 3 \mathrm{~K} 9 \mathrm{me} 3$ peptides.

\subsubsection{The MBT proteins MBTR-1 and LIN-61 interact with H3K9me3}

To identify C. elegans H3K9me3 interaction proteins, peptide pull-down experiments were performed $(2.3 .7 ; 2.5 .9)$. C. elegans extract (based on Cheseman protocol, 2.5.8.1) was incubated with either $\mathrm{H} 3 \mathrm{~K} 9 \mathrm{me} 0$ or $\mathrm{H} 3 \mathrm{~K} 9 \mathrm{me} 3$ peptides that were coupled to agarose beads. The bound fraction of each peptide was separated by SDS-PAGE and proteins were analyzed by LC-MS/MS (2.3.3). The statistical program R was used to select proteins specifically present in the H3K9me3 bound fraction by subtracting the protein data set obtained for the $\mathrm{H} 3 \mathrm{~K} 9 \mathrm{me} 0$ bound fraction from the protein data set obtained for the $\mathrm{H} 3 \mathrm{~K} 9 \mathrm{me} 3$ bound fraction. 34 proteins, identified with at least two unique peptides, were found to interact with the H3K9me3 peptide (for complete list see 6.2.1). LIN-61 and MBTR-1, the two MBT proteins of C. elegans (see 1.2 and alignment in Figure 3-3), were among these. 23 unique peptides were identified for LIN-61 (sequence coverage 45\%) and six unique peptides were identified for MBTR-1 (sequence coverage 12\%) (Figure 3-1). Surprisingly, HPL-2 that was shown to interact with $\mathrm{H} 3 \mathrm{~K} 9 \mathrm{me} 3$ [117] was not found in this experiment.

A

$$
\begin{aligned}
& \text { Identified protein: LIN-61 } \\
& \text { MSEFLKIVRANKKSDRKLDKTYLWESYLHQFEKG } \\
& \text { KTSFIPVEAFNRNLTVNFNECVKEGVIFETVVHDY } \\
& \text { DKNCDSIQVRWFARIEKVCGYRVLAQFIGADTKF } \\
& \text { WLNILSDDMFGLANAAMSDPNMDKIVYAPPLAINE } \\
& \text { EYQNDMVNYVNNCIDGEIVGQTSLSPKFDEGKALL } \\
& \text { SKHRFKVGQRLELLNYSNSTEIRVARIQEICGRRM } \\
& \text { NVSITKKDFPESLPDADDDRQVFSSGSQYWIDEGSF } \\
& \text { FIFPVGFAAVNGYQLNAKKEYIEHTNKIAQAIKNGE } \\
& \text { NPRYDSDDVTFDQLAKDPIDPMIWRKVKVGQKFE } \\
& \text { LIDPLAQQFNNLHVASILKFCKTEGYLIVGMDGPDA } \\
& \text { LEDSFPIHINNTFMFPVGYAEKYNLELVPPDEFKGT } \\
& \text { FRWDEYLEKESAETLPLDLFKPMPSQERLDKFK } \\
& \text { VGLRLEAADMCENQFICPATVKSVHGRLINVNFD } \\
& \text { GWDEEFDELYDVDSHDILPIGWCEAHSYVLQPPKK } \\
& \text { YNY }
\end{aligned}
$$

B

Identified protein: MBTR-1

MEKSSSNFIQNGKRDHGGKLRQYNYKLEEAEYRYF TEERLFYRRRNPVEKIAQRIPKPQIEGTFTWSDELRC NYDGNTQFLPVEALEGCLPLEKLNQHLKPGFRLEV VVRPSLDPSITTKSPEIRWFGEVTAVCGFYVAIKFVG ELNRRPCWFHMLSEDIFDIGSGLKQDPAMKWLQYR PLSLLKPMQCPKFWRRGSTPAPPVPRPTEEILDEFQA ELHENRISEPKIFDQLRHLAHRPSRFRLNQRVELLN YLEPTEIRVARILRILGRRLMVMVTAQDYPEDLPSV EAKDRQVQHENVEFWVDESSFFLFPVGFAMINGLR TKATEGYLEHSRRIAEGSGSYHKDDVTFEQLFAGKP DISAEKLNLLKVGQKFELLDPLSDLRQSFCVATIRK ICKTPGFLIISPDETESDDESFPIHIDNHFMHPVGYAE KFGIKLDRLAGTEPGKFKWEGYLKEKQAEKIPDEM LRPLPSKERRHMFEFGRVLEAVGQNETYWISPASV EEVHGRTVLIEFQGWDSEFSELYDMDSHDLLPAGW CEFFNFKLRHPVLPVNDPNAENGEYD

Figure 3-1: Identification of LIN-61 and MBTR-1 as H3K9me3 peptide binding proteins 
(A) Protein sequence of LIN-61 (B) Protein sequence of MBTR-1 (A; B) Unique peptides identified in $\mathrm{MS} / \mathrm{MS}$ analysis of $\mathrm{H} 3 \mathrm{~K} 9 \mathrm{me} 3$ binding fraction are indicated in red.

To verify the interaction of LIN-61 and MBTR-1 with H3K9me3 peptides in an independent experiment, both factors were in vitro transcribed and translated as MYC-tagged proteins in rabbit reticulocyte lysate and used for peptide pull-down experiments with $\mathrm{H} 3 \mathrm{~K} 9 \mathrm{me} 0$ and $\mathrm{H} 3 \mathrm{~K} 9 \mathrm{me} 3$ peptides bound to beads (2.3.6; 2.3.7). Washing steps were performed in buffer containing the twofold salt concentration $(300 \mathrm{mM} \mathrm{KCl})$ as compared to the pull-down experiments shown elsewhere in this study to strengthen the binding conditions. The protein fractions bound to the peptides were analyzed by western blotting using $\alpha \mathrm{MYC}$ antibody. Both MYC-tagged proteins specifically interacted with H3K9me3 but not with $\mathrm{H} 3 \mathrm{~K} 9 \mathrm{me} 0$ peptide (Figure 3-2). These results confirm the interaction of LIN-61 and MBTR-1 with H3K9me3 peptide. They show that recombinant LIN-61 and MBTR-1 bind to H3K9me3 irrespective of context of $C$. elegans extract. The identification of MBT proteins interacting with a tri-methylated peptide was unexpected, as all up to date investigated human or Drosophila MBT proteins discriminate against tri-methyl lysine (1.2).

A

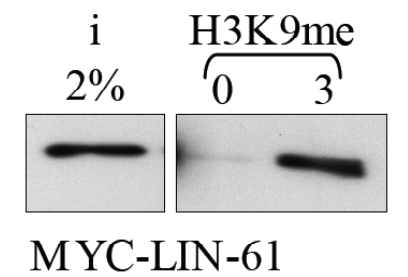

B

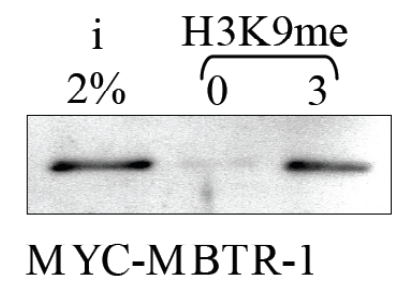

Figure 3-2: Verification of LIN-61 and MBTR-1 as H3K9me3 peptide binding proteins

Peptide pull-down experiments using $\mathrm{H} 3 \mathrm{~K} 9 \mathrm{me} 0$ and $\mathrm{H} 3 \mathrm{~K} 9 \mathrm{me} 3$ peptides were performed with (A) in vitro translated MYC-tagged LIN-61 and (B) in vitro translated MYC-tagged MBTR-1. Proteins bound to peptides were eluted by boiling in protein sample buffer, separated by SDS-PAGE and analyzed by western blotting using $\alpha \mathrm{MYC}$ antibody. i: input

\subsection{Characterization of LIN-61 H3K9me3 interaction}

Motivated by the unexpected finding that the $C$. elegans MBT proteins interact with tri-methylated $\mathrm{H} 3 \mathrm{~K} 9$ peptides, methyl mark binding by $C$. elegans MBT proteins on the basis of LIN-61 interaction with methylated histone tail peptides was further characterized. 


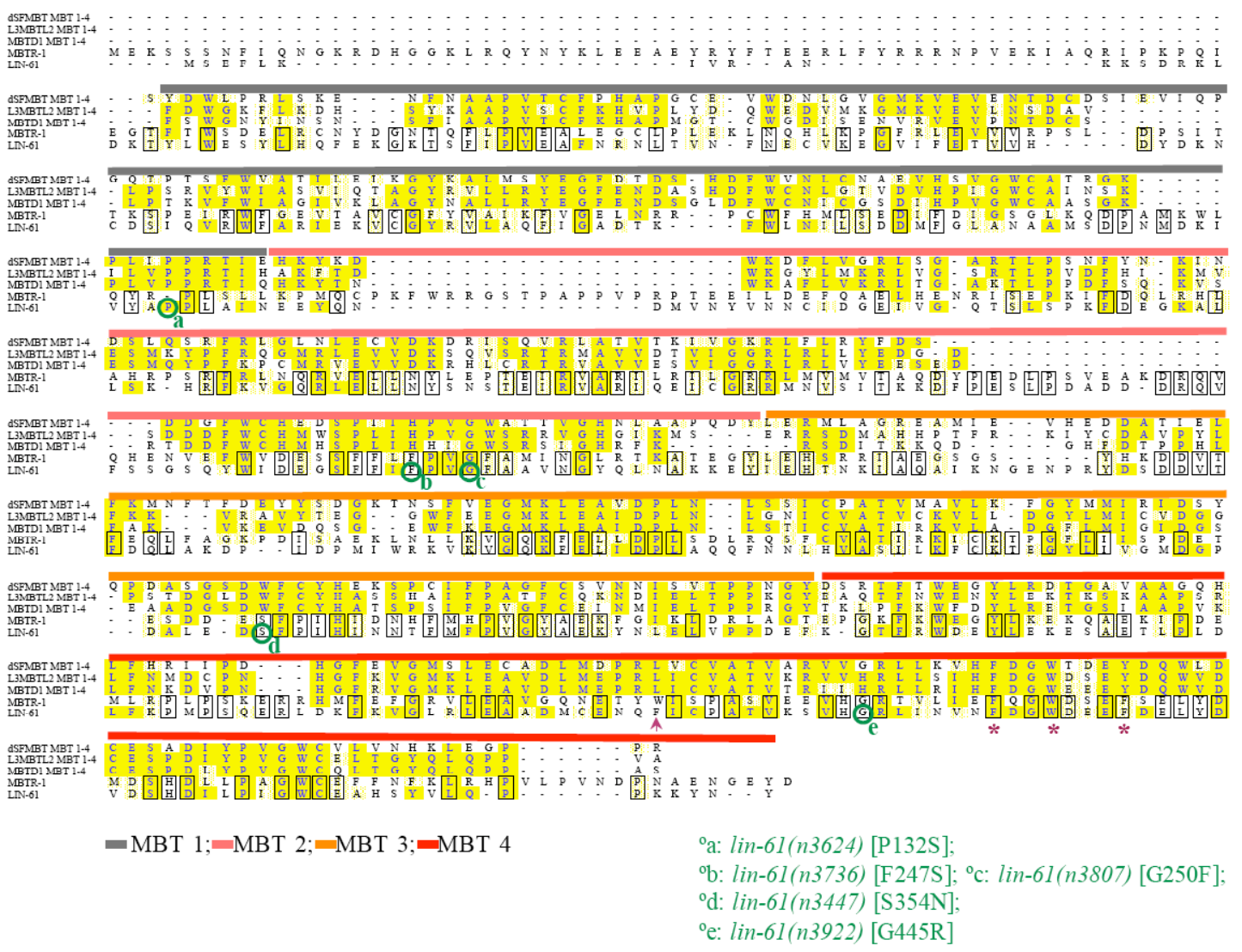

Figure 3-3: Alignment of LIN-61 and MBTR-2 protein sequences with the MBT repeats of fourMBT repeat proteins

ClustalW alignment of LIN-61 and MBTR-1 protein sequences with the protein sequence of the MBT repeats two to four of dSFMBT [56], hL3MBTL2 [57], hMBTD1 [63]. Dark yellow boxes indicate identity to consensus sequence of a sub alignment from the MBT repeats of dSFMBT, L3MBTL2, MBTD1. Light yellow boxes indicate similarities. Colored boxes above the alignment mark regions of MBT repeats deduced from [56, 57, 63] (see legend). LIN-61 and MBTR-1 are 36\% identical, (black boxes). Asterisks mark conserved aromatic cage residues (see 1.2.1.2; 3.2.3.2.). Green circles indicate residues mutated in particular lin-61 alleles (see 3.4.1.1).

\subsubsection{Binding to H3K9me3 is an autonomous function of LIN-61}

I could show that not only endogenous LIN-61 interacts with H3K9me3 peptides but the corresponding in vitro translated MYC-tagged counterpart also interacts with these peptides (3.1.1). However, the interaction of LIN-61 with the heterochromatic mark might be mediated by other factors within the $C$. elegans extract or the reticulocyte lysate and therefore could be indirect. To elucidate if LIN-61 directly binds to the H3K9 tri-methyl mark, recombinant MBP tagged protein was expressed in E. coli, amylose affinity purified, and used for peptide pull down experiments. Figure 3-4 (A) shows a Coomassie stained SDS-PAGE gel of amylose affinity purified MBP-tagged LIN-61. 
In a pull-down experiment that was performed without the presence of blocking reagents, MBP-tagged LIN-61 bound to H3K9me3 peptide but in the same way it interacted with unmodified peptide (Figure 3-4 B). However, specific binding to trimethylated peptide compared to $\mathrm{H} 3 \mathrm{~K} 9 \mathrm{me} 0$ was achieved when the peptide protein incubation step of the pull-down experiment was carried out in $25 \% \mathrm{v} / \mathrm{v}$ C. elegans extract (blocking buffer (C. elegans extract)) or in $0.5 \% \mathrm{w} / \mathrm{v}$ low-fat dry milk (blocking buffer (milk)) (Figure 3-4 C and D).

One assumption to explain the gain in H3K9me3 binding specificity of LIN-61 upon presence of milk or $C$. elegans extract is that the amylose affinity purified MBPtagged LIN-61 sample might not be homogenous, but instead might consist of different LIN-61 pools with differing binding specificity for non- and tri-methylated H3K9 peptides. Supplementation milk or $C$. elegans extract might abrogate the binding of an unspecific interacting LIN-61 pool to H3K9me0 peptides.

To dissect if the observed inconclusive properties of LIN-61 regarding peptide specificity might be ascribed to a heterogeneous pool of recombinant LIN-61, amylose affinity purified LIN-61 was further purified by gel filtration chromatography.

Two different MBP-tagged LIN-61 pools were separated by size. The main part of the amylose affinity purified MBP-tagged LIN-61 was eluted from the gel filtration column after a low retention volume close to the exclusion volume indicating a high molecular weight multimerization/aggregation state of MBP-tagged LIN-61 (Fr. 1) (Figure 3-4 E). The second peak was eluted after a larger retention volume indicating that the MBP-tagged LIN-61 in this much smaller protein fraction (Fr. 2) is in a lower molecular weight state as compared to fraction one and monomeric (Figure 3-4 E).

To analyze if the protein fractions of either peak behave differently regarding specificity for tri-methylated versus unmethylated $\mathrm{H} 3 \mathrm{~K} 9$ peptides, peptide pull-down experiments were performed with both fractions separately (Figure 3-4 F, G). MBPtagged LIN-61 of fraction one (Fr. 1) bound specifically to H3K9me3 peptides when the pull-down reaction was supplemented with milk but bound to both peptides equally strong when no blocking reagent was added to the pull-down reaction (Figure 3-4 F). The protein in fraction one thus behaved the same as the original non-further purified amylose purified MBP-tagged LIN-61. In contrast, monomeric MBP-tagged LIN-61 from fraction two (Fr. 2) bound specifically to H3K9me3 but not to 
$\mathrm{H} 3 \mathrm{~K} 9 \mathrm{me} 0$ peptides regardless of the presence of milk during the pull-down reaction (Figure 3-4 F).

These results show that recombinant purified LIN-61 has the intrinsic capacity to interact specifically with $\mathrm{H} 3 \mathrm{~K} 9 \mathrm{me} 3$ peptides (Figure 3-4 F, G) and $\mathrm{H} 3 \mathrm{~K} 9 \mathrm{me} 3$ interaction is therefore an autonomous function of LIN-61.

Furthermore, they show that MBP-tagged LIN-61 when it is purified in one step by amylose purification consists of two LIN-61 pools and that a blocking buffer has to be added to peptide pull-down experiments to avoid unspecific peptide interaction of multimerized/aggregated LIN-61 in peptide pull-down experiments.

To further uncover the binding characteristics of LIN-61, pull-down experiments that are shown in the following sections and for which recombinant expressed and purified protein was used were performed in either blocking buffer (milk) or blocking buffer (C. elegans extract) when indicated. 
$\mathbf{A}$

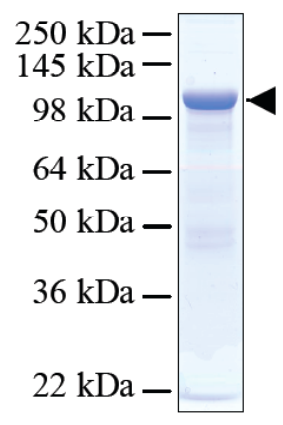

$\mathbf{E}$

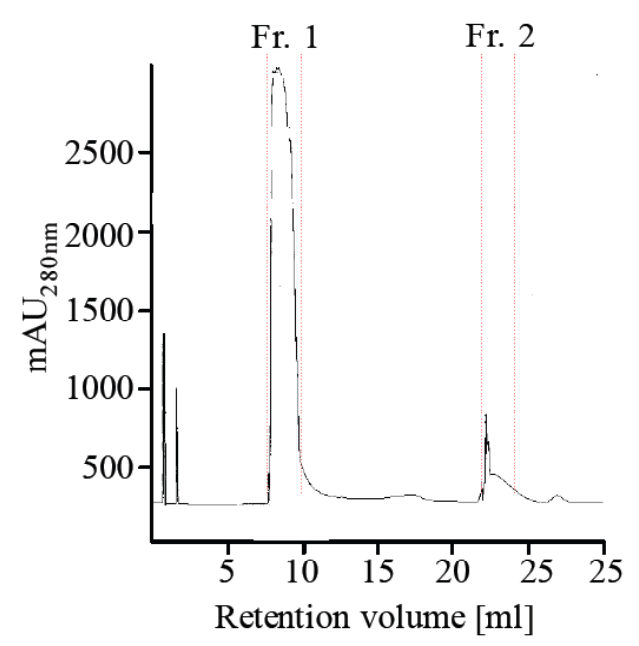

B

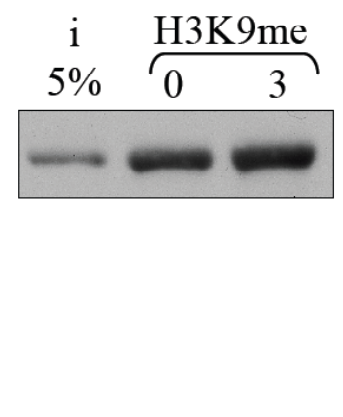

C

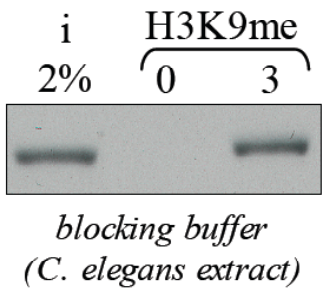

D

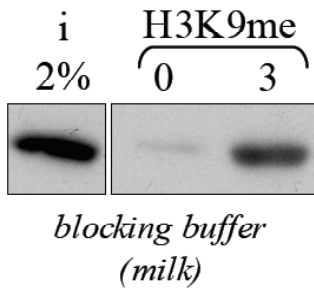

$\mathbf{F}$

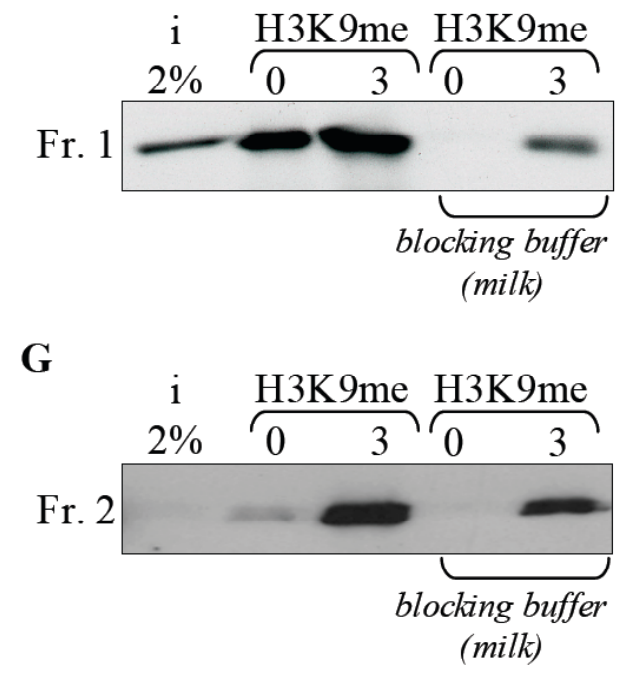

Figure 3-4: recombinant MBP-tagged LIN-61 interacts specifically with H3K9me3

(A) Recombinant MBP-tagged LIN-61 expressed in E. coli and affinity purified with amylose resin was separated by SDS-PAGE and visualized by Coomassie Blue staining (arrowhead). Molecular weight markers are indicated on the left. (B-D) Peptide pull-down experiments using H3K9me0 and H3K9me3 peptides were performed with amylose affinity purified MBP-tagged LIN-61. The peptide protein incubation step was carried out in blocking buffer (C. elegans extract) or in blocking buffer (milk) as indicated. Peptide bound fractions were separated by SDS-PAGE and visualized by western blotting using $\alpha \mathrm{MBP}$ antibodies. (E) Amylose affinity purified MBP-tagged LIN-61 was separated on a Superdex 200 gel filtration column. Elution profile and pooled fractions are shown. (F, G) Peptide pulldown experiments were performed as in (B-D) with (F) MBP-tagged LIN-61 of the high molecular weight fraction (Fr. 1) or with (G) MBP-tagged LIN-61 of the monomeric low molecular weight fraction (Fr. 2). i: input 


\subsubsection{LIN-61 binds specifically to H3K9 methylated peptides}

LIN-61 specifically interacts with $\mathrm{H} 3 \mathrm{~K} 9 \mathrm{me} 3$ peptide but discriminates against the non-methylated state of the same peptide. To analyze whether LIN-61 shows sequence specific binding, pull-down reactions with different methylated peptides corresponding to distinct histone methylation marks were performed. For these studies I used $C$. elegans extract and in parallel the recombinant HIS-tagged and purified three MBT repeats of L3MBTL1. The recombinant HIS-tagged protein composed of the three MBT repeats of L3MBTL1 $(1.2 .1 .1[49,58])$ interacted with all mono-methylated peptides irrespective of the surrounding sequence as shown in the western blot analysis of Figure 3-5 (A). In contrast, endogenous LIN-61, bound only methylated H3K9 peptides. Because I found LIN-61 bound stronger to $\mathrm{H} 3 \mathrm{~K} 9 \mathrm{me} 3$ compared to $\mathrm{H} 3 \mathrm{~K} 9 \mathrm{me} 1$, I also analyzed $\mathrm{H} 3 \mathrm{~K} 9 \mathrm{me} 2$ interaction in separate pull-down experiments. As shown in Figure 3-5 (B), LIN-61 out of C. elegans extract interacted equally well with $\mathrm{H} 3 \mathrm{~K} 9 \mathrm{me} 2$ and $\mathrm{H} 3 \mathrm{~K} 9 \mathrm{me} 3$ peptides $(\mathrm{H} 3 \mathrm{~K} 9 \mathrm{me} 3=$ H3K9me2 >> H3K9me1 >> H3K9me0).

A

$\begin{array}{lllll}\mathrm{H} 3 & \mathrm{H} 3 & \mathrm{H} 3 & \mathrm{H} 3 & \mathrm{H} 4\end{array}$

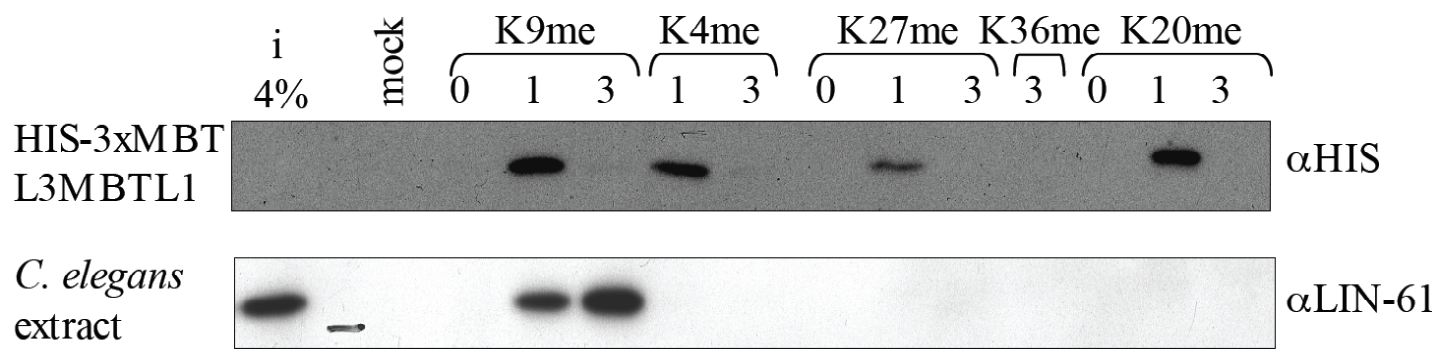

B

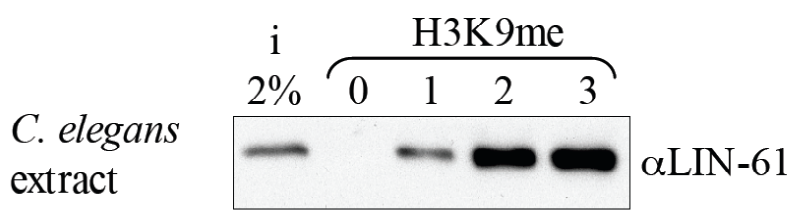

Figure 3-5: Endogenous LIN-61 interacts specifically with the H3K9 methyl mark

(A) Peptide pull-down experiments were performed using the indicated peptides with either recombinant purified HIS-tagged 3xMBT repeats of L3MBTL1 or with C. elegans protein extract. Peptide bound protein fractions were separated by SDS-PAGE and analyzed by western blotting using the indicated antibodies. (B) Peptide pull-down experiment was performed with $C$. elegans protein extract as in (A). i: input; mock: pull-down reaction that was performed without peptide 
To further sustain these findings, I also analyzed the methyl mark binding behavior of recombinant LIN-61.

Using either non-, mono- or tri-methylated peptides with differing sequences in pulldown experiments with recombinant amylose purified MBP-tagged LIN-61 revealed that the sequence specific interaction with methylated $\mathrm{H} 3 \mathrm{~K} 9$ peptides with preference for the higher methylated state observed for endogenous LIN-61 is a direct function of LIN-61 (Figure 3-6 A, B).

In a peptide pull-down competitor assay (Figure 3-6 C), sequence specific binding and clear discrimination against the non-methylated state was further confirmed for recombinant MBP-tagged LIN-61. This experiment showed that the unspecific binding fraction of MBP-tagged LIN-61 loses binding to bead coupled H3K9me0 peptides when $\mathrm{H} 3 \mathrm{~K} 9 \mathrm{me} 0, \mathrm{H} 2 \mathrm{~K} 20 \mathrm{me} 0$ or $\mathrm{H} 4 \mathrm{~K} 20 \mathrm{me} 3$ peptides are used as competitor peptide. This result equals the results obtained when milk or extract was used as blocking reagent (compare with Figure 3-4 and Figure 3-5 A). In contrast, H3K9me3 was the only competitor peptide that could impaire the recovery of LIN-61 on bead coupled H3K9me3 peptide in the peptide pull-down competitor assay.

$\mathbf{A}$

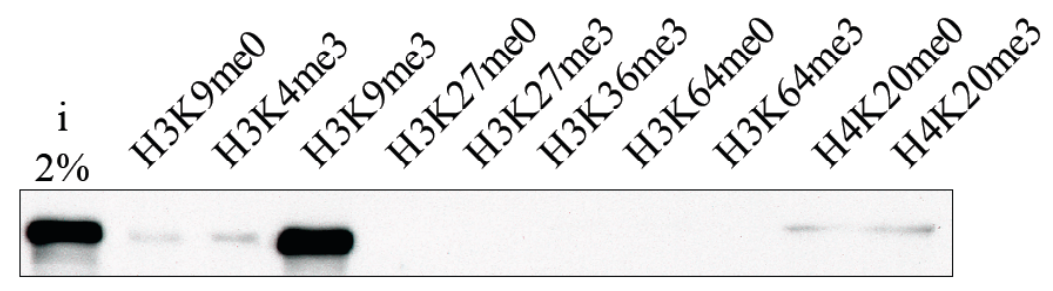

B

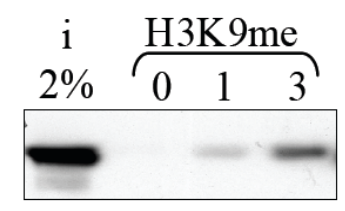

C

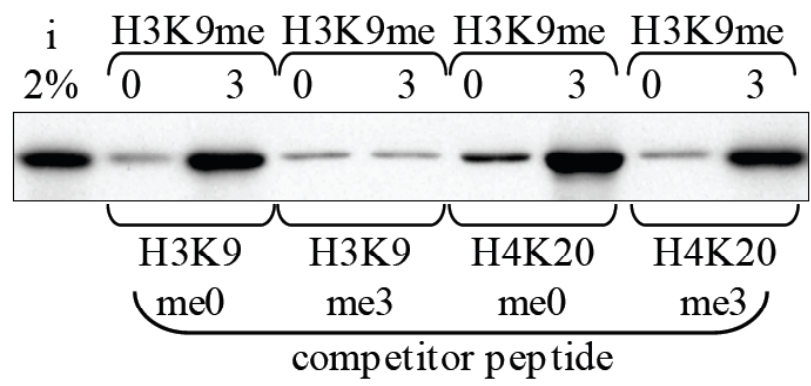

Figure 3-6: Recombinant LIN-61 specifically binds H3K9me3 peptides

(A) Peptide pull-down experiments were performed using the indicated peptides bound to agarose beads and amylose affinity purified recombinant MBP-tagged LIN-61. The peptide protein incubation step was carried out in blocking buffer (milk). Peptide bound fractions were separated by SDS-PAGE and visualized by western blotting using $\alpha \mathrm{MBP}$ antibodies. (B) Peptide pull-down experiments were performed using $\mathrm{H} 3 \mathrm{~K} 9 \mathrm{me} 0$, H3K9me1 and $\mathrm{H} 3 \mathrm{~K} 9 \mathrm{me} 3$ peptides as described in (A). (C) Peptide pulldown experiments using either $\mathrm{H} 3 \mathrm{~K} 9 \mathrm{me} 0$ or $\mathrm{H} 3 \mathrm{~K} 9 \mathrm{me} 3$ peptides were performed as in (A) but the 
peptide protein incubation step was carried out without blocking buffer but together with $500 \mu \mathrm{g}$ (100fold excess compared to bound peptide on beads) competitor peptide as indicated. i: input

\subsubsection{Three MBT repeats and a functional aromatic cage motif are necessary for LIN-61/H3K9me3 interaction}

From the results described in the previous sections I concluded that LIN-61 has discriminative binding affinities for differently methylated histone tail peptides. This is in sharp contrast to the binding characteristics of all other so far investigated MBT proteins. Hence, although LIN-61 is mainly composed of MBT repeats and does not contain any other recognizable domains, its binding mechanism to peptides must vary from the mechanism of other MBT proteins.

To better understand the binding mechanism of LIN-61 to the H3K9me3 mark and to dissect similarities and differences compared to other MBT proteins, functional analysis with different LIN-61 mutations were performed using the binding competence to $\mathrm{H} 3 \mathrm{~K} 9 \mathrm{me} 3$ peptides in pull-down experiments as a read-out.

\subsubsection{MBT repeats two to four of LIN-61 are necessary for $\mathrm{H} 3 \mathrm{~K} 9 \mathrm{me}$ interaction}

Crystal structures of the MBT repeats of several proteins of human and Drosophila origin have revealed that the MBT repeats of these proteins are folded into one unit with a high level of interdigitation between the individual MBT domains. The structures of the four MBT repeats of dSFMBT, L3MBTL2 and MBTD1 indicate that four MBT repeats might in general be arranged similarly with the last three MBT repeats forming a propeller like structure and the first MBT sticking out of this structure (1.2.1.3).

Based on the assumption that the MBT repeats of LIN-61 are similarly composed as in the other four MBT repeat proteins, I hypothesized that the first MBT repeat of LIN-61 might not be essential for the overall composition of the residual MBT repeats and thus not essential for methyl mark binding. To test this hypothesis, a truncated protein containing only the three C-terminal MBT repeats of LIN-61 was expressed as a GST-tagged protein in E. coli and affinity purified by glutathione sepharose resin. Peptide pull-down experiments showed that the three last MBT repeats of LIN-61 were sufficient for the protein to specifically interact with $\mathrm{H} 3 \mathrm{~K} 9 \mathrm{me} 3$ peptide (Figure 
3-7 A). In contrast, further truncated GST-tagged LIN-61 proteins missing MBT repeats two, three and/or four did not bind at all to $\mathrm{H} 3 \mathrm{~K} 9 \mathrm{me} 3$ in peptide pull-down experiments (Figure 3-7 C-D). These results indicate that the MBT repeats two to four are required for $\mathrm{H} 3 \mathrm{~K} 9 \mathrm{me} 3$ methyl mark binding of LIN-61.

A

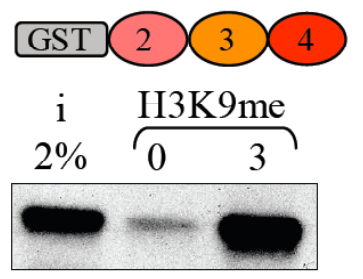

B

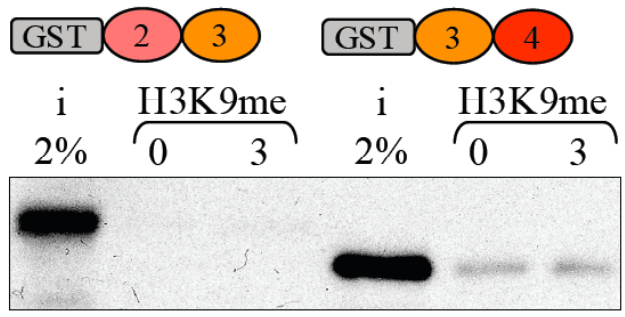

C

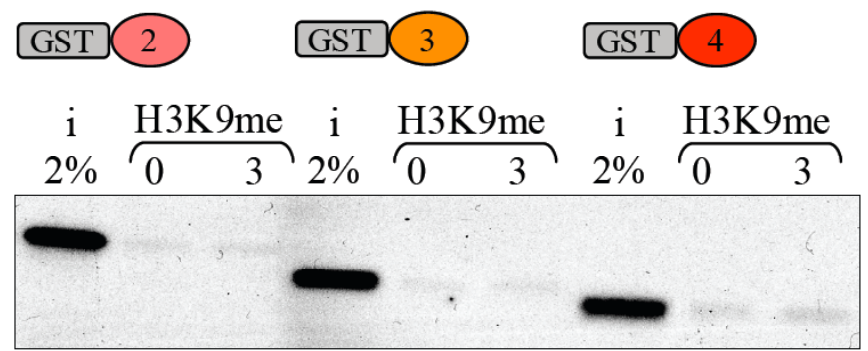

Figure 3-7: MBT repeats two to four of LIN-61 are required for H3K9me3 peptide binding

(A - C) Peptide pull-down experiments were performed using $\mathrm{H} 3 \mathrm{~K} 9 \mathrm{me} 0$ and $\mathrm{H} 3 \mathrm{~K} 9 \mathrm{me} 3$ peptides with purified GST-tagged LIN-61 deletion mutant proteins as schematically indicated above the corresponding pull-down experiment result. The protein sequence of the deletion mutant proteins corresponds to MBT repeat sequences of LIN-61 as indicated in Figure 3-3. The peptide protein incubation step was carried out with blocking-solution (C. elegans extract). Peptide bound fractions were separated by SDS-PAGE and visualized by western blotting using $\alpha$ GST antibody. i: input

\subsubsection{A functional aromatic cage motif is necessary for LIN-61 H3K9me3 binding}

All structurally analyzed MBT repeats contain one functional binding pocket per MBT repeat unit, which selectively binds mono- and di-methyl lysine. As discussed in 1.2.1.1, three conserved aromatic residues that build the aromatic cage motif combine with a conserved aspartate to build the functional binding pocket.

To identify the methyl-lysine binding pockets of LIN-61, the protein sequence of the MBT core domains of each LIN-61 MBT domain were aligned with the protein sequences of functional binding pocket bearing MBT core domains of other MBT proteins (Figure 3-8 A). Only MBT core domain four in LIN-61 contains all three 
conserved aromatic residues that might form a cage and in addition the conserved aspartate.

To investigate whether the conserved aspartate in MBT domain four (D428) and the conserved aromatic cage residues within the same domain (F452 W455 F459) are implicated in binding of the methyl mark H3K9me3, LIN-61 point mutants with specific amino acids exchanged to alanine were analyzed in peptide pull-down experiments (Figure 3-8 C-G). The proteins were expressed with a MYC-tag in rabbit reticulocyte lysate using the TNT system. The binding affinity of the LIN-61 point mutant proteins towards $\mathrm{H} 3 \mathrm{~K} 9 \mathrm{me} 3$ was evaluated by peptide pull-down experiments (Figure 3-8 B).

The analysis revealed that binding of the LIN-61 D428A to H3K9me3 was in fact of lower affinity as compared to the wild-type protein but not abolished (Figure 3-8 B; C). However, a triple mutation of LIN-61 in which all three conserved aromatic residues were exchanged to alanine (F452A W455A F459A) abolished binding of LIN-61 to H3K9me3 peptides (Figure 3-8 E-G).

The analysis of LIN-61 single point mutants with only one of the three aromatic residues exchanged to alanine revealed that the $\mathrm{F} 452 \mathrm{~A}$ mutation has only a minor effect on the H3K9me3 binding capacity of LIN-61, while the W455A mutation abolished and the F459A mutation strongly impaired H3K9me3 interaction (Figure 3-8 C).

These results indicate that LIN-61 like other MBT proteins possesses only one functional binding pocket. In addition, two conserved aromatic cage residues, essential for methyl lysine binding in other MBT repeats are essential for H3K9me3 interaction in LIN-61. 
A

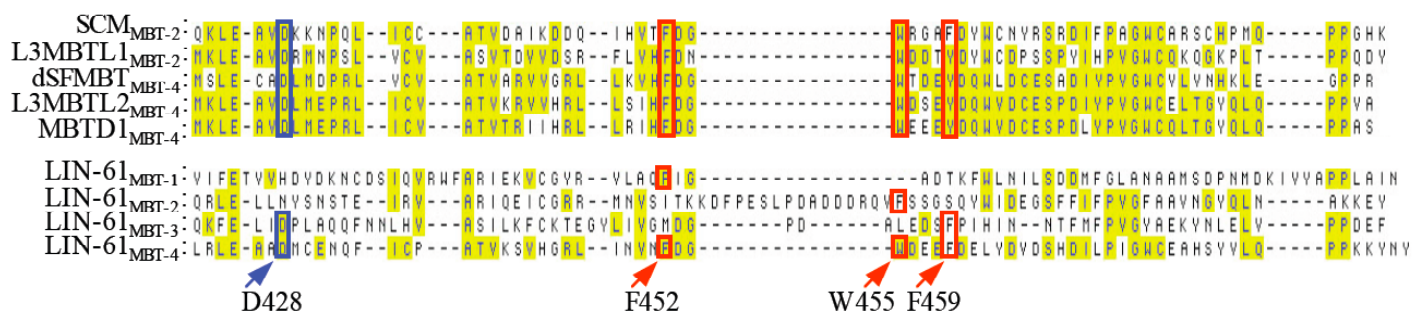

B

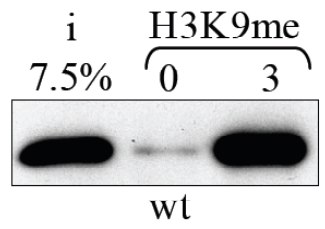

$\mathbf{E}$

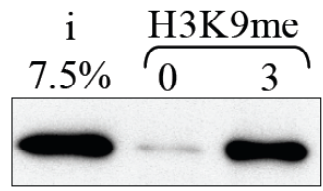

F452A
C

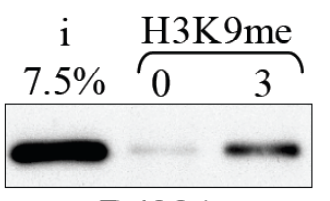

D428A

$\mathbf{F}$

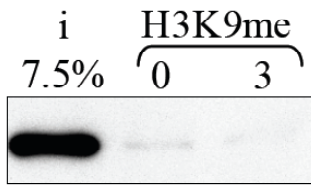

W455A
D

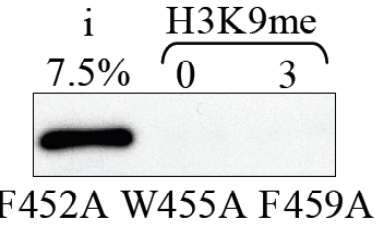

G

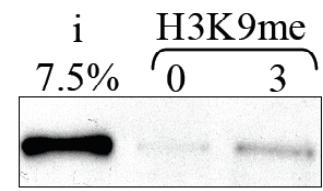

F459A

Figure 3-8: D428A mutation results in slight and W455A or F459A mutation in strong impairment of LIN-61 H3K9me3 interaction

(A) Alignment of the protein sequences of MBT core domains of indicated MBT proteins that interact with methylated histone tail peptides with the four MBT core domains of LIN-61. Positions corresponding to cage-forming residues in $\mathrm{SCM}_{\mathrm{MBT}-2}$ core, $\mathrm{L}_{3 \mathrm{MBTL}} 1_{\mathrm{mbt}-2}$ core, $\mathrm{dSFMBT} \mathrm{MBT}_{-4}$ core and MBTD $1_{\text {MBT- } 4 \text { core }}$ are marked with a red box and the positions of the conserved aspartate are marked with a blue box. Positions of the conserved aromatic cage residues and the aspartate within the MBT core domains of LIN-61 are depicted with boxes using the same colors as for the other MBT-repeat protein core domains. N-terminal arm regions of the MBT domain are not included in the alignment. (B - G) Peptide pull-down experiments using $\mathrm{H} 3 \mathrm{~K} 9 \mathrm{me} 0$ and $\mathrm{H} 3 \mathrm{~K} 9 \mathrm{me} 3$ peptides were performed with in vitro translated MYC-tagged LIN-61 point mutant proteins. The particular amino acid exchange and its position in the LIN-61 protein sequence are indicated. Proteins bound to peptides were eluted by boiling in protein sample buffer, separated by SDS-PAGE and analyzed by western blotting using aMYC antibody. i: input

\subsection{Characterization of lin-61 and mbtr-1 deletion mutants}

To discover biological functions of LIN-61 and MBTR-1 in C. elegans, phenotypic analysis of lin-61 and mbtr-1 deletion mutants were performed.

\subsubsection{Description of lin-61 and mbtr-1 alleles used in this study}

For phenotypic analysis of lin-61 associated phenotypes, lin-61(tm2649) mutant worms were assayed. The strain was obtained from the National BioResource Project and backcrossed five times to wild-type N2 worms to diminish background mutations. $\operatorname{tm} 2649$ is a deletion allele with a 672 bp deletion within the genomic sequence of lin- 
61 (schematically illustrated in Figure 3-9 A). The deletion was confirmed by sequencing the truncated coding sequence of lin- 61 that was obtained by RT-PCR performed on total RNA of lin-61(tm2649) worms (see also Figure 3-9 B). Although mRNA is still made in the lin-61 deletion mutant (Figure 3-9 B), no protein was detected by western blot analysis of crude lin-61(tm2649) worm lysate using $\alpha$ LIN-61 (guinea pig) antibodies. Since the LIN-61 peptide used for raising the guinea pig antibodies maps to the very N-terminus of LIN-61 that should be present if a truncated protein were made, I conclude that $\operatorname{tm} 2649$ is a null allele.

A

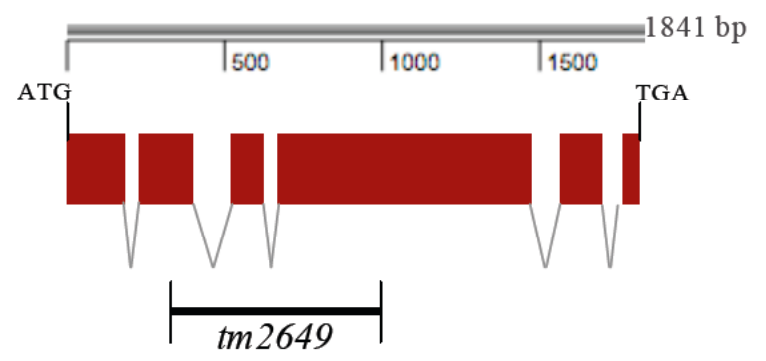

B

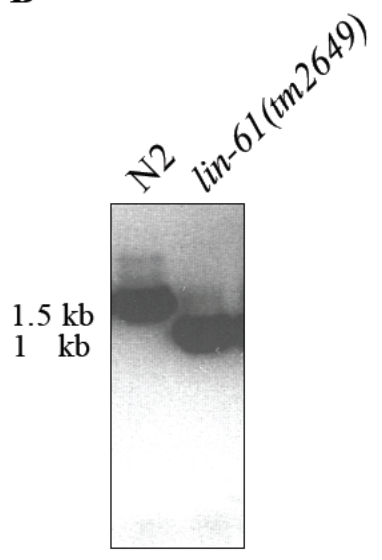

C

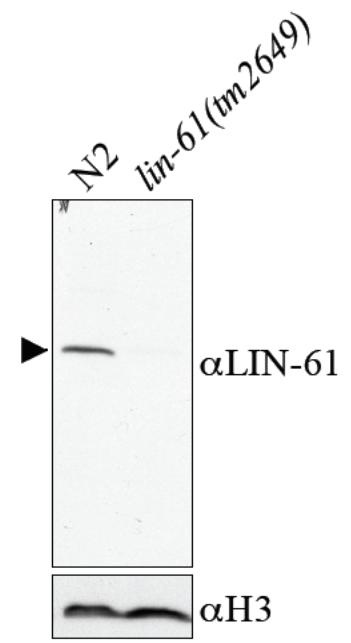

Figure 3-9: Characterization of the lin-61 null allele $t m 2649$

(A) lin-61 gene structure ranging from translation initiation codon (ATG) to the termination codon (TGA). Boxes indicate coding sequence. The bar indicates the genomic region deleted in $t m 2649$. (B) Ethidium bromide stained agarose gel showing RT-PCR fragments, amplified with primers that border the coding sequence of lin-61. RT-PCR was performed on total RNA of N2 or lin-61 (tm2649) worms. (C) Western blot analysis of $\mathrm{N} 2$ and lin-61 (tm2649) crude $C$. elegans lysates using $\alpha \mathrm{LIN}-61$ (guinea pig) antibodies. Arrowheads indicate endogenous wild-type LIN-61. $\alpha \mathrm{H} 3$ western blot performed as loading control

For phenotypic analysis of mbtr-1, mbtr-1(n4775) mutant worms were assayed. The corresponding strain MT15643, obtained from R. Horvitz and characterized in Harrison et al. [100], was backcrossed three times to wild-type N2 before analysis. The 1878 bp deletion within the genomic sequence of mbtr-1 (illustrated in Figure 3-10 A) was confirmed by sequencing the truncated coding sequence of $m b t r-1$ that was obtained by RT-PCR performed on total RNA of $m b t r-1(n 4775)$ worms (see also Figure 3-10 B). Figure 3-10 B shows that truncated mbtr-1 mRNA is present in mbtr$1(n 4775)$ mutant worms. Nonetheless the $n 4775$ allele is a presumptive null allele as 
the hypothetical truncated MBTR-1 protein resulting from the truncated mbtr-1 mRNA would result a in frame shift after amino acid 165. Therefore most of the MBT repeats would be missing (Figure 3-10 C).

A

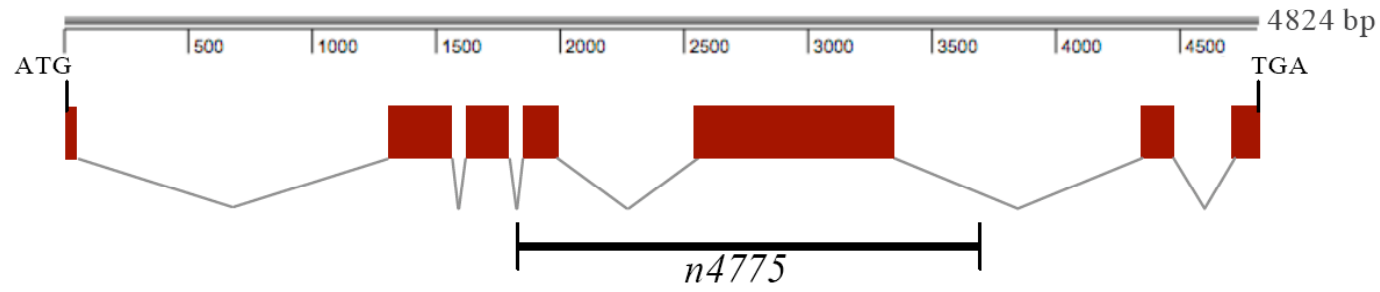

B

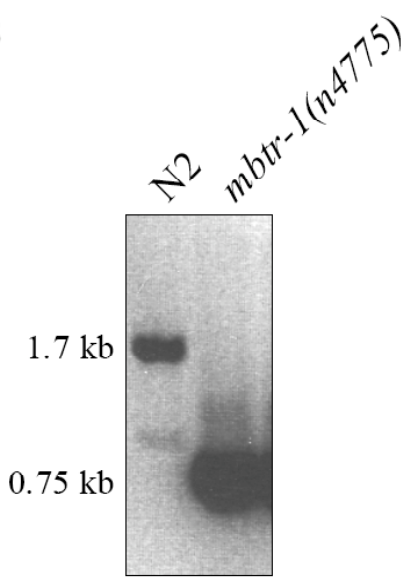

C

Hypothetical truncated MBTR-1 protein

MEKSSSNFIQNGKRDHGGKLRQYNYKLEEAEYRYFTE ERLFYRRRNPVEKIAQRIPKPQIEGTFTWSDELRCNYD GNTQFLPVEALEGCLPLEKLNQHLKPGFRLEVVV RPSLDPSITTKSPEIRWFGEVTAVCGFYVAIKFVGE LNRRPCWFHMLSEDIFDIGSSVVSLKPSAKTRPTGYHRPPSKK YTVERCSSSFRD GIPNFPSFMIWTPTIYFOPVGANSSISNFDIPYFO

(247 aa)

Figure 3-10: Description of the mbtr-1 allele $n 4775$

(A) $m b t r$-1gene structure ranging from translation initiation codon (ATG) to the termination codon (TGA). Boxes indicate coding sequence. A bar indicates the genomic region deleted in $n 4775$. (B) Ethidium bromide stained agarose gel showing RT-PCR fragments, amplified with primers that border the coding sequence of $m b t r-1$. RT-PCR was performed on total RNA of N2 or mbtr-1(n4775) worms. (C) Protein sequence of a hypothetical truncated MBTR-1 protein based on the mbtr-1 genomic sequence change in mbtr-1(n4775). The underlined sequence indicates parts of MBT domain one; the italic sequence indicates a sequence not corresponding to wild-type MBTR-1 that would results from the frame shift in $n 4775$.

\subsection{2 lin-61 and mbtr-1 single and double mutants show no apparent morphological changes}

Neither the lin-61 nor the mbtr-1 single mutation caused any obvious phenotype at either $20^{\circ} \mathrm{C}$ or $24.5^{\circ} \mathrm{C}$. The morphology of lin- 61 or mbtr-1 mutant worms was indistinguishable from wild-type N2 animals (Figure 3-11 A - C). Since lin-61 and mbtr-1 encode the only MBT proteins in C. elegans, I analyzed mbtr-1 lin-61 double mutant worms. However, no morphological defects could be detected (Figure 3-11 D). These findings are in agreement with parallel studies by Harrison et al. [100]. 
A

\section{N2}

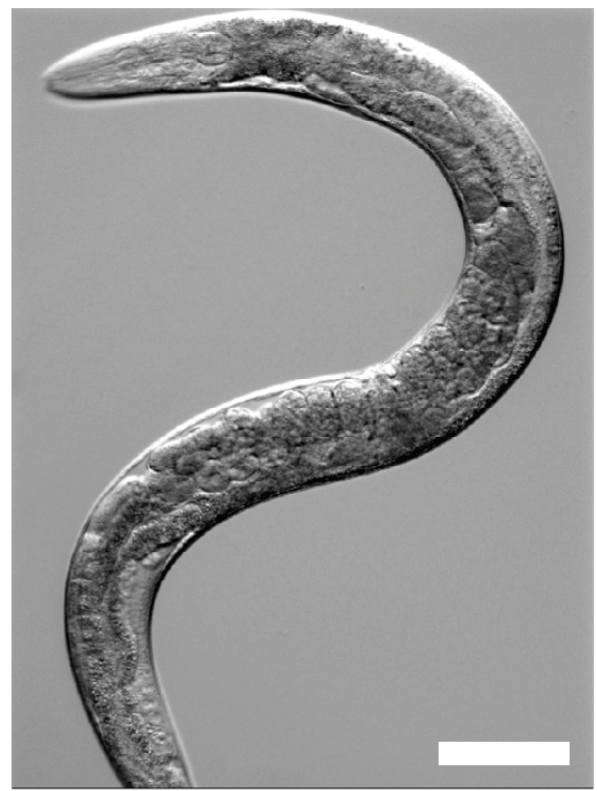

C

mbtr-1

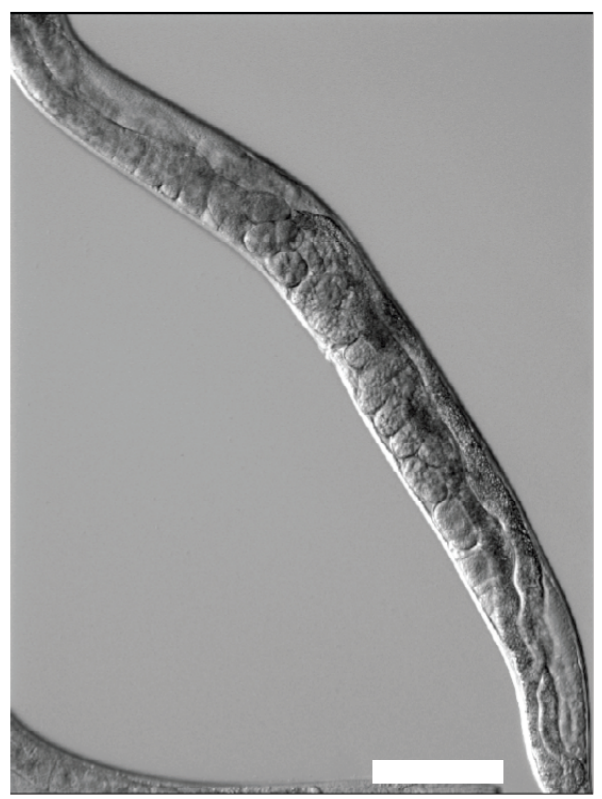

B

$$
\text { lin-61 }
$$

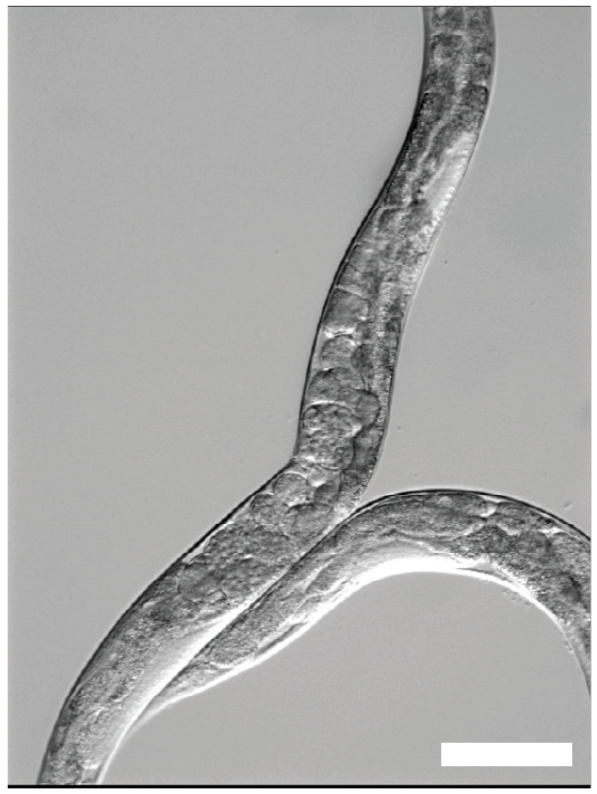

D

mbtr-1 lin-61

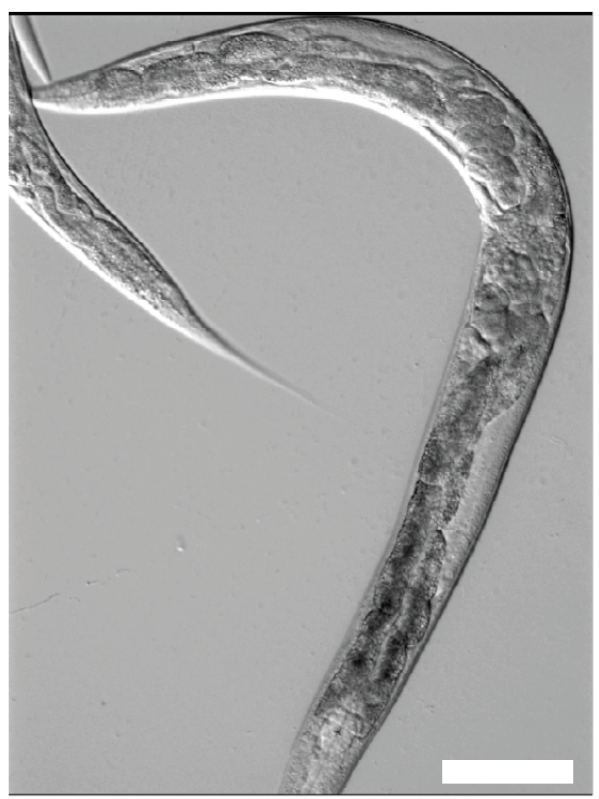

Figure 3-11: lin-61 and mbtr-1 single or lin-61 mbtr-1 double mutants show no morphological changes

Representative images of worms of (A) wild-type N2, (B) lin-61(tm2649), (C) mbtr-1(n4775) and (D) mbtr-1(n4775) lin-61(tm2649) genotype are shown. Animals were grown at $24.5^{\circ} \mathrm{C}$ for $\sim 72$ hrs before DIC images were taken. Scale bar represents $100 \mu \mathrm{m}$. 


\subsection{3 lin-61 but not mbtr-1 is a synMuvB gene}

In a genome wide RNAi screen lin-61 was identified as synMuvB gene [101].

While the present study was ongoing, Harrison et al. [100] showed that diverse lin-61 alleles cause a synMuv phenotype in a synMuvA mutant background.

As outlined in 3.3.1, phenotypic analysis of lin-61 performed in the present study is on the basis of the null allele $\operatorname{tm} 2649$ that has not been characterized in previous studies. The analysis of lin-61(tm2649) in the loss-of-function background (either mutation or RNAi) of the synMuvA gene lin-15A revealed that the tm2649 allele causes a strong synMuv phenotype. The findings further confirmed the data of lin-61 being a synMuvB gene (Table 3-1; Figure 3-12).

Table 3-1: lin-61 causes a synthetic multivulva phenotype in combination with lin-15A

\begin{tabular}{|c|c|c|}
\hline Genotype & $\% \mathbf{M u v} \pm \mathrm{SD}$ & (n) \\
\hline $\operatorname{lin}-15 A(R N A i)$ & $0 \pm 0$ & $(>750)$ \\
\hline $\operatorname{lin}-15 A(n 767)$ & $0 \pm 0$ & (976) \\
\hline$m b t r-1(n 4775)$ & $0 \pm 0$ & $(>200)$ \\
\hline $\operatorname{lin}-61(t m 2649)\left(20^{\circ} \mathrm{C}\right)$ & $0.1 \pm 0.2$ & (914) \\
\hline $\operatorname{lin}-61(\operatorname{tm} 2649) *$ & $0.3 \pm 0.2$ & $(1197)$ \\
\hline mbtr-1(n4775); lin-15A(RNAi) & $0 \pm 0$ & $(1040)$ \\
\hline $\operatorname{lin-61(tm2649);~lin-15A(RNAi)}$ & $94 \pm 2.1$ & $(783)$ \\
\hline $\operatorname{lin-61}(\operatorname{tm} 2649) ; \operatorname{lin}-15 A(n 767)\left(20^{\circ} \mathrm{C}\right)$ & $78.3 \pm 14$ & $(825)$ \\
\hline $\operatorname{lin}-61(\operatorname{tm} 2649) ; \operatorname{lin}-15 A(n 767)$ & $99.9 \pm 0.2$ & $(833)$ \\
\hline \multicolumn{3}{|c|}{$\begin{array}{l}\text { All animals were raised at } 24.5^{\circ} \mathrm{C} \text { unless otherwise stated and the } \mathrm{F} 1 \text { offspring of three hermaphrodites } \\
\text { per trial were assayed. Three trials per strain were conducted. The Muv phenotype was analyzed using } \\
\text { a dissecting microscope and animals were scored as Muv if one or more ectopic ventral protrusions } \\
\text { (pseudovulvae) were observed. lin-15A(RNAi) was performed by feeding Ht115(DE3) bacteria } \\
\text { ZK678.1 (containing the lin-15A cDNA cloned into p14440-Dest). SD: Standard deviation; n: total } \\
\text { number of worms observed; *: strain that was raised on control feeding plates using Ht1 15(DE3) } \\
\text { bacteria containing the empty L4440 vector. }\end{array}$} \\
\hline
\end{tabular}


A

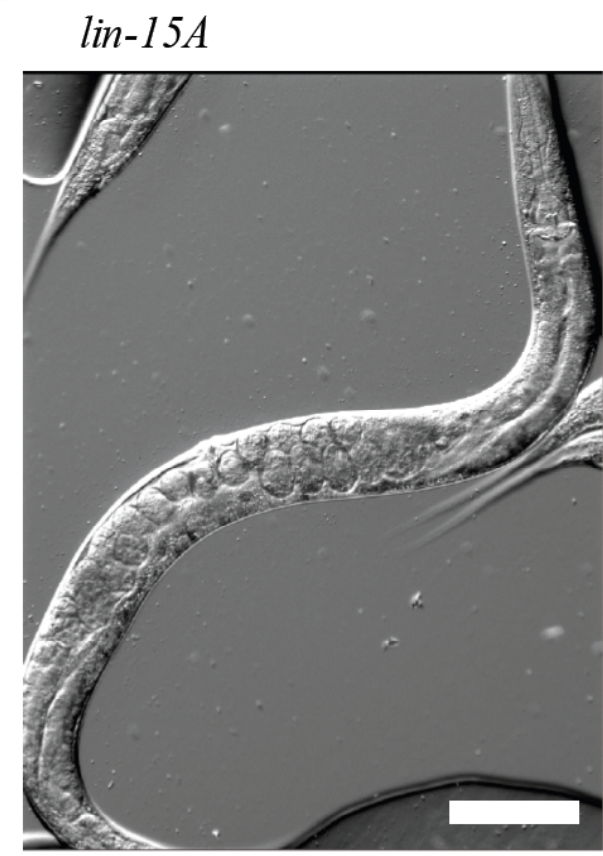

B

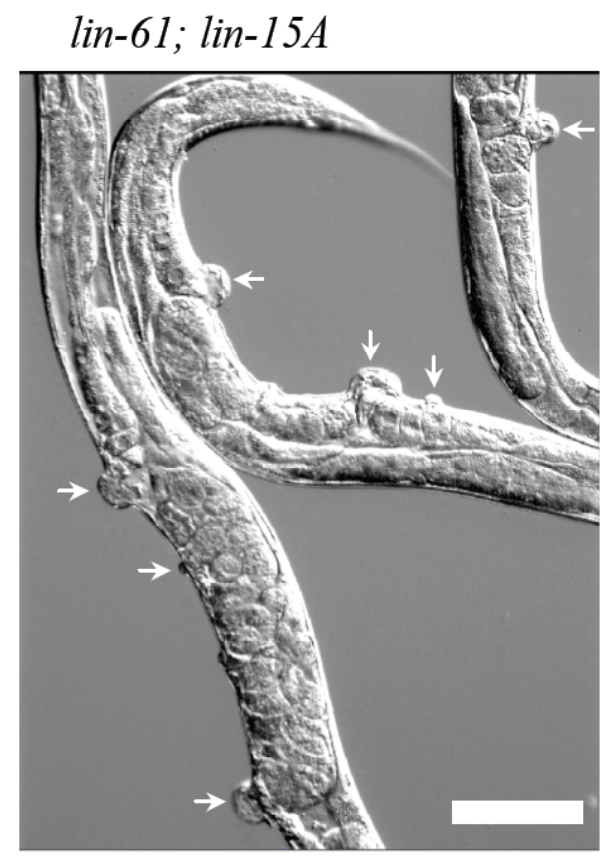

Figure 3-12: lin-61; lin-15A causes a synMuv phenotype

Representative images of worms of (A) lin-15A(n767) and (B) lin-61(tm2649); lin-15A(n767) genotype grown at $24.5^{\circ} \mathrm{C}$ for $72 \mathrm{hrs}$ before DIC images were taken. (For comparison of lin-61; lin-15A with lin61 see Figure 3-11 B) Scale bar represents $100 \mu \mathrm{m}$. Arrows indicate pseudovulvae.

It is thought that synMuvA and synMuvB genes function as negative regulators of ectopic vulval cell fate development by concerted repression of lin-3 expression (see 1.3.1.2). It was shown by Cui et al. [89] that whereas synMuvA and synMuvB single mutants do not cause elevated lin-3 mRNA levels, the lin-3 expression level in synMuvA; synMuvB double mutants is increased. To analyze if lin-61; lin-15A cause increased lin-3 expression, lin-3 mRNA levels of N2, lin-61, lin-15A and lin-61; lin$15 \mathrm{~A}$ worms at the L3 stage were quantified and normalized to rpl-26 mRNA levels (Figure 3-13). While the single mutations showed a slight increase in lin-3 expression compared to wild-type $N 2$, the lin-3 mRNA level in lin-61; lin-15A mutant worms was more than doubled compared to wild-type worms. In support of this result, an elevated lin-3 expression level was also reported for lin-61(n3909); lin-15A(n433) worms while the present study was ongoing [119]. I conclude that LIN-61 exerts a repressive function on $\operatorname{lin}-3$ expression within the synMuvB pathway. 


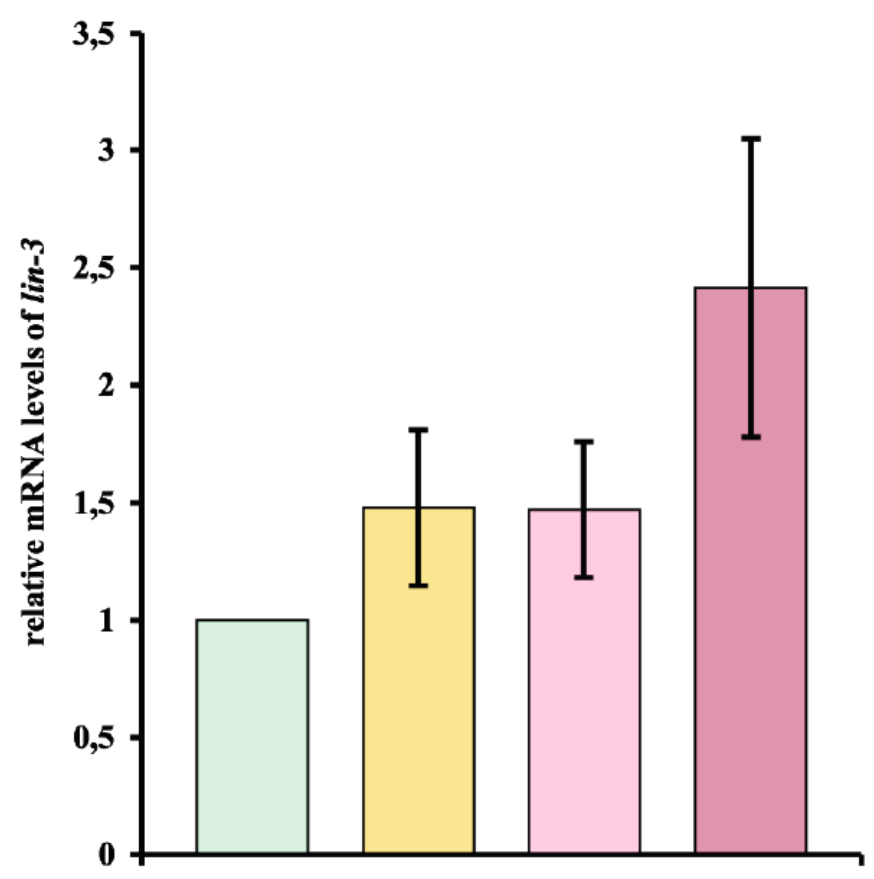

Figure 3-13: lin-3 mRNA level is elevated in lin-61; lin-15A

Relative fold changes of lin-3 mRNA levels of L3 worms of indicated genotypes (see color code in legend) raised at $20^{\circ} \mathrm{C}$. The mean $2^{-(\Delta \Delta \mathrm{Ct})}$ values were calculated based on real-time PCR triplicates of two RNA isolations from independent worm growth. Error bars represent standard deviation.

In contrast to lin-61, mbtr-1 is not a synMuvB gene. The $n 4775$ allele does not cause a synMuv phenotype by loss-of-function of lin-15A (Table 3-1). Similar results were obtained by Harrison et al. [119].

\subsubsection{Genetic interaction of lin-61 with met-2 and hpl-2 causes a synMuv phenotype}

As synMuvB factor, LIN-61 is involved in the repression of ectopic vulval cell fate development and a repressive function of LIN-61 on lin-3 expression could be demonstrated (3.3.3). Several genes that encode proteins with functions in negative regulation of chromatin are among the reported synMuvB genes (1.3.1.3). My results show that LIN-61 also belongs to this category, as interaction with H3K9me3 could be shown. Interestingly, a H3K9 HMT (MET-2) was identified as a synMuvB factor [112] and in addition HPL-2, a HP1 homologue [113]. HPL-2 is the only other protein within the synMuvB pathway besides LIN-61 that was shown to bind to H3K9me3 in peptide pull-down experiments (Figure 3-14 A and [117]).

To dissect the function of LIN-61 within the synMuvB pathway in the context of MET-2 and HPL-2, lin-61; met-2 and lin-61; hpl-2 double mutant C. elegans were generated and analyzed for synthetic phenotypes (Table 3-2). 
Table 3-2: lin-61 causes a synMuv phenotype by genetic interaction with $m e t-2$ or $h p l-2$ at $24.5^{\circ} \mathrm{C}$

\begin{tabular}{|c|c|c|c|c|}
\hline \multirow[b]{2}{*}{ Genotype } & \multicolumn{2}{|l|}{$20^{\circ} \mathrm{C}$} & \multicolumn{2}{|l|}{$24.5^{\circ} \mathrm{C}$} \\
\hline & $\% \mathbf{M u v} \pm \mathrm{SD}$ & (n) & $\% \mathbf{M u v} \pm \mathrm{SD}$ & (n) \\
\hline met-2 & $0 \pm 0$ & $(695)$ & $0.4 \pm 0.2$ & (875) \\
\hline$h p l-2$ & $0 \pm 0$ & (1150) & $2.2 \pm 0.7$ & (1068) \\
\hline mbtr-1 lin-61 & $0 \pm 0$ & $(>400)$ & $0 \pm 0$ & $(>400)$ \\
\hline mbtr-1; hpl-2 & $0 \pm 0$ & $(738)$ & $1.4 \pm 0.8$ & (819) \\
\hline lin-61; met-2 & $0.8 \pm 0.7$ & $(482)$ & $12.9 \pm 3.1$ & (507) \\
\hline lin-61; hpl-2 & $0.8 \pm 0.5$ & $(1551)$ & $84.4 \pm 4$ & (1416) \\
\hline
\end{tabular}

All animals scored were raised at $20^{\circ} \mathrm{C}$ or $24.5^{\circ} \mathrm{C}$ as indicated. Three trials of independent worm growth per genotype were conducted. The Muv phenotype was analyzed using a dissecting microscope and animals were scored as Muv if one or more ectopic ventral protrusions (pseudovulvae) were observed. SD: Standard deviation; n: total number of worms analyzed

Interestingly, genetic interaction was observed in both cases. The lin-61; met-2 double mutation causes a mild synthetic Muv phenotype and the lin-61; hpl-2 double mutation causes a strong synthetic Muv phenotype at $24.5^{\circ} \mathrm{C}$ (Table 3-2; Figure 3-14 C).

These results indicate that LIN-61 acts redundantly with HPL-2 and to some extent redundantly with MET-2 to control vulval cell fate decision at $24.5^{\circ} \mathrm{C}$.

In addition, I analyzed if mbtr-1 lin-61 causes a synMuv phenotype but no genetic interaction could be detected (Table 3-2). 
A

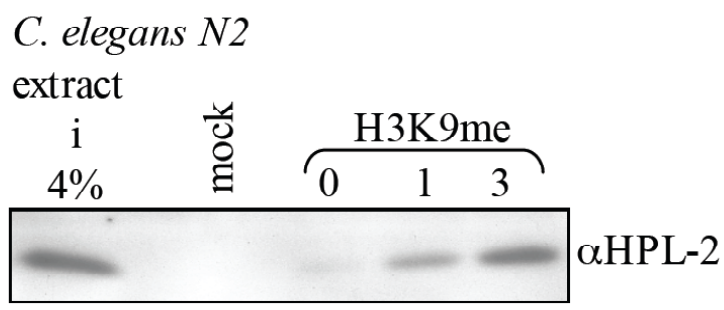

C. elegans N2

C
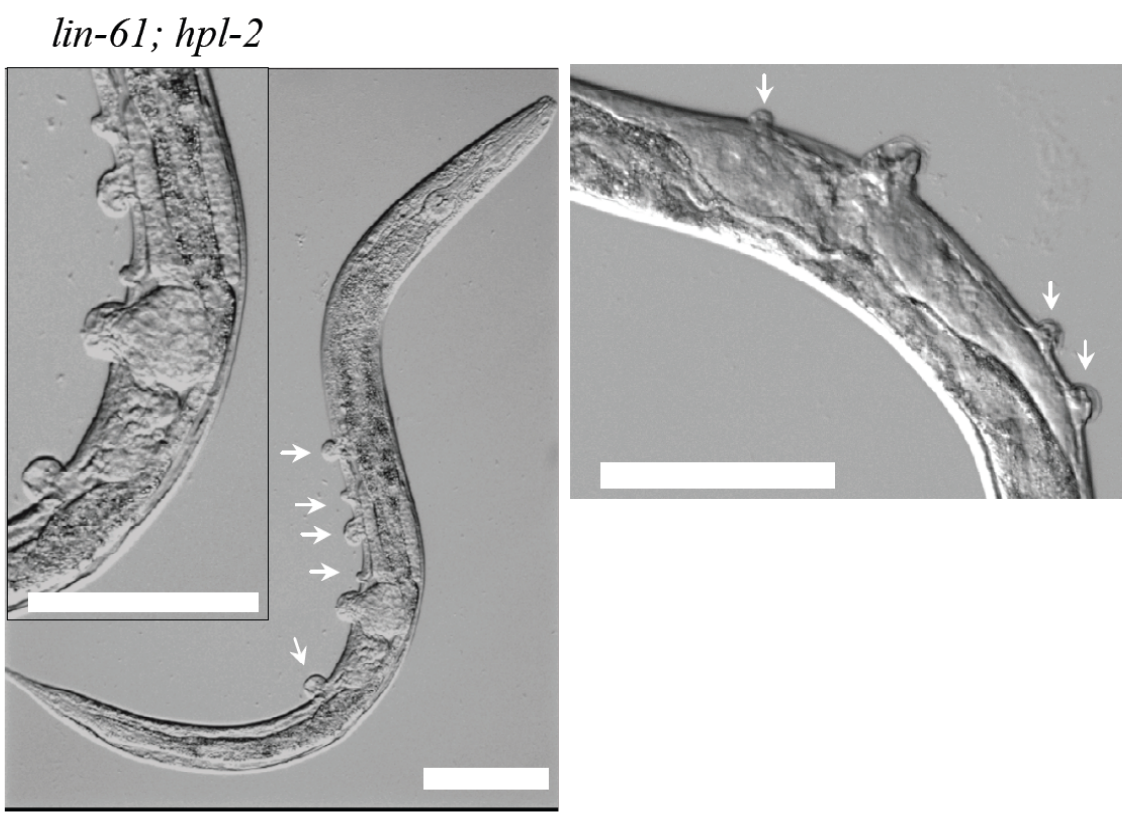

Figure 3-14: lin-61; $h p l-2\left(24.5^{\circ} \mathrm{C}\right)$ are Muv and have disorganized gonadal structures

(A) Peptide pull-down experiments were performed using $\mathrm{H} 3 \mathrm{~K} 9 \mathrm{me} 0$ and $\mathrm{H} 3 \mathrm{~K} 9 \mathrm{me} 3$ peptides with $C$. elegans protein extract. Peptide bound protein fractions were separated by SDS-PAGE and analyzed by western blotting. Binding of endogenous HPL-2 was visualized using $\alpha$ HPL-2 antibodies. i: input; mock: pull-down reaction that was performed without peptide $(\mathbf{B}, \mathbf{C})$ Representative images of worms of (B) hpl-2(tm 1489) and (C) lin-61(tm2649); hpl-2(tm 1489) genotype are shown. Animals were grown at $24.5^{\circ} \mathrm{C}$ for $72 \mathrm{hrs}$ before DIC images were taken. (For comparison of lin-61; hpl-2 with lin-61 see Figure 3-11 B.) Scales bar represents $100 \mu \mathrm{m}$. Arrows indicate pseudovulvae. 


\subsubsection{Synergistic effects of lin-61 with met-2 and hpl-2 on fertility}

Wild-type adult hermaphrodites contain two U-shaped gonad arms with mitotic germ cell nuclei in the distal region and oocytes in the proximal part. A uterus with developing embryos is separated from the gonad arms by spermstoring spermathecae. For lin-61 single mutant worms, no obvious defects in gonadal structures were observed (Figure 3-11 B).

For $h p l-2$ mutant worms it is reported that they show gonadal defects as single mutants. Schott et al. showed by analyzing the localization of GFP-expression of particular gene marker in worms grown at $24{ }^{\circ} \mathrm{C}$ that a low percentage of $h p l-2$ single mutants fail to correctly differentiate distal tip cells (DTC) (35\%), sheath cells $(24.2 \%)$ and spermathecal cells (39\%) indicating defects in somatic gonad development $[114,144]$. In line with these results I detected $\mathrm{hpl}-2$ mutant worms at $24.5^{\circ} \mathrm{C}$ that showed defects in gonad morphology and did not contain any oocytes or embryos (Figure 3-14 B, worm on right) next to hpl-2 mutant worms with no obvious gonadal defects (Figure 3-14 B, worm on left).

However in addition to a strong Muv phenotype, the gonad structures appeared to be strongly disorganized in most lin-61; hpl-2 double mutant worms at $24.5^{\circ} \mathrm{C}$ and often no oocytes, no embryos and/or no elongated gonad arms could be detected in adult lin-61; hpl-2 hermaphrodites (Figure 3-14 C). The significant increase in gonadal defects in a lin-61; hpl-2 background indicate that both factors are involved in gonadal development, having partly redundant functions. The morphological gonadal defects of lin-61; $h p l-2$ animals were not further dissected in more detail in the present study but might contain defects of the germline and/or oocyte maturation besides defects of the somatic gonad that were already detected in $h p l-2$ single mutants [114].

Encouraged by the detected enhanced gonadal defects caused by lin- 61 in an hpl-2 mutant background, I decided to perform brood size assays as several gonadal defects might have an impact on brood size and/or cause sterility (Figure 3-15).

lin-61 does not result in sterility but in a mild reduced brood size at $20^{\circ} \mathrm{C}$. However lin-61 caused occasional sterility $(2 \%)$ and a $\sim 30 \%$ reduced mean brood size compared to wild-type at $24.5^{\circ} \mathrm{C}$.

Both, $h p l-2$ and met-2 single mutations caused a mild sterile phenotype and a reduced mean brood size at $20^{\circ} \mathrm{C}$ and at $24.5^{\circ} \mathrm{C}$. 
Interestingly lin-61 caused in $\mathrm{hpl}-2$ as well as in met-2 mutant background synergistic effects on sterility on brood size.

Consistent with the morphological gonadal defects detected in lin-61; $h p l-2$ worms at $24.5^{\circ} \mathrm{C}$ (Figure $3-14 \mathrm{C}$ ), I detected a strong sterile phenotype (98\%, one worm laid two eggs) caused by lin-61; hpl-2 at this temperature. But synergism was also detectable at $20^{\circ} \mathrm{C}$. At this temperature $22 \%$ sterile animals were detected and the mean brood size of lin-61; $h p l-2$ was $\sim 75 \%$ reduced compared to $h p l-2$.

The lin-61; met-2 double mutant was $47 \%$ sterile at $24.5^{\circ} \mathrm{C}$ while the met-2 single mutation caused $8 \%$ sterility at this temperature. In addition there was a synergistic reduction in mean brood size for non-sterile lin-61; met-2 worms compared to the single mutants at $24.5^{\circ} \mathrm{C}(\sim 63 \%$ reduced compared to met-2). Inconsistent results however were obtained at $20^{\circ} \mathrm{C}$ as lin-61; met-2 caused no sterility at this temperature whereas for met-2 $7 \%$ sterility was detected. Nonetheless a synergistic reduction in mean brood size of about $29 \%$ compared to met-2 was detected for lin-61; met-2 at $20^{\circ} \mathrm{C}$.

Interestingly, $m b t r-1$ caused neither a phenotypic effect on sterility or brood size as a single mutant nor in a lin-61 or hpl-2 mutant background at both temperatures analyzed. 
A

\begin{tabular}{lrrrrrr}
\hline \hline \multirow{2}{*}{ Genotype } & \multicolumn{2}{c}{$\mathbf{2 0}^{\circ} \mathrm{C}$} & & \multicolumn{2}{c}{$\mathbf{2 4 . 5}^{\circ} \mathrm{C}$} \\
\cline { 2 - 3 } \cline { 5 - 7 } wild-type (N2) & \multicolumn{2}{c}{$\%$ Sterile (n) } & & \multicolumn{2}{c}{$\%$ Sterile (n) } \\
lin-61 & 0 & $(29)$ & & 0 & $(38)$ \\
hpl-2 & 0 & $(50)$ & & 2 & $(44)$ \\
lin-61; hpl-2 & 13 & $(30)$ & & 25 & $(28)$ \\
met-2 & 22 & $(54)$ & & 98 & $(60)$ \\
lin-61; met-2 & 7 & $(30)$ & & 8 & $(26)$ \\
mbtr-1 & 0 & $(17)$ & & 47 & $(15)$ \\
mbtr-1 lin-61 & 0 & $(30)$ & & 0 & $(38)$ \\
mbtr-1; hpl-2 & 0 & $(29)$ & & 0 & $(36)$ \\
\hline \hline
\end{tabular}

B

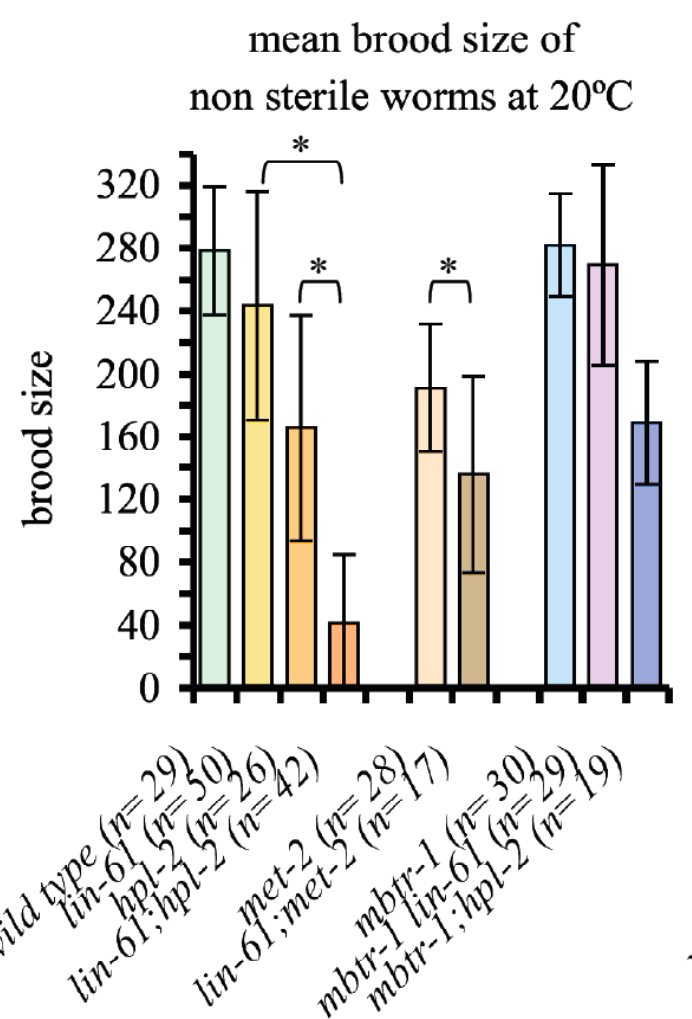

C

mean brood size of non sterile worms at $24.5^{\circ} \mathrm{C}$

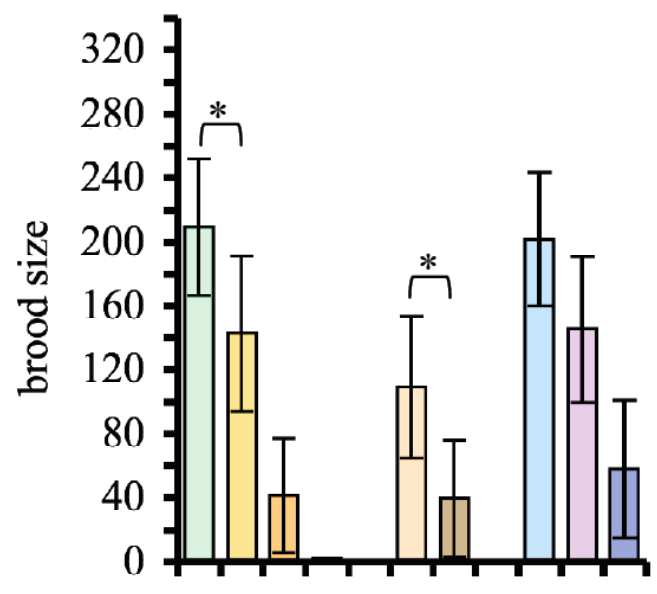

Figure 3-15: lin-61 causes reduced mean brood size at $24.5^{\circ} \mathrm{C}$ and is synergistic to $\mathrm{hpl}-2$ and $m e t-2$ regarding sterility rate and mean brood size

(A) The table depicts frequency of sterility determined for the indicated genotypes at the indicated temperature. n: number of worms analyzed $(\mathbf{B} ; \mathbf{C})$ Mean brood size of non sterile worms of indicated genotype at (B) $20^{\circ} \mathrm{C}$ or at (C) $24.5^{\circ} \mathrm{C}$. Error bars show standard deviation; Asterisks and brackets mark strains that differ significantly in brood size with a $p$-value of $<0,001$ ( $p$-values were calculated with a standard Student t-test); n: number of worms analyzed 
In addition to a slightly reduced brood size, lin-61 mutant worms had a reduced life span compared to wild-type $\mathrm{N} 2$ worms at $20^{\circ} \mathrm{C}$ and at $24.5^{\circ} \mathrm{C}$. Representative life span curves in Figure 3-16 (A; B) illustrate that in a worm population of lin-61, more worms died at earlier time points compared to a N2 population. Calculation of mean life span revealed that in average lin-61 mutant worms died $2.5\left(20^{\circ} \mathrm{C}\right)$ and 3.6 $\left(24.5^{\circ} \mathrm{C}\right)$ days earlier than N2 worms (Figure 3-16 C; D).

The loss-of-function mutation of $h p l-2$ only caused a reduction in mean life span at $24.5^{\circ} \mathrm{C}$ whereas met- 2 worms showed a reduced mean life span at both temperatures tested (Figure 3-16). The reduction in mean life span at $24.5^{\circ} \mathrm{C}$ of $h p l-2$ animals is comparable to the reduction in mean life span of lin-61 worms, whereas met-2 showed more severe defects. The mean life span of met- 2 was found to be 5.6 days $\left(20^{\circ} \mathrm{C}\right)$ or 6.5 days $\left(24.5^{\circ} \mathrm{C}\right)$ shorter compared to the mean life span of $\mathrm{N} 2$.

Interestingly, although the life span of $h p l-2$ worms was in average not reduced compared to wild-type at $20^{\circ} \mathrm{C}$, analysis of mean lifespan of lin-61; $h p l-2$ double mutant worms showed that lin-61; $h p l-2$ worms died on average $\sim 2$ days earlier than lin-61 single mutant worms at $20^{\circ} \mathrm{C}$ or at $24.5^{\circ} \mathrm{C}$.

For lin-61; met-2, there was no significant decrease in mean life span compared to met-2.

Overall, these data indicate that LIN-61 has more general functions in C. elegans besides a function in the synMuvB pathway. LIN-61 has unique functions as well as redundant functions with HPL-2 and MET-2 regarding fertility and life span.

Importantly, no defects were detected for mbtr-1 mutant worms. mbtr-1 mutant worms were found to live on average slightly longer than $\mathrm{N} 2$ worms at $20^{\circ} \mathrm{C}$. As in the brood size assay,there was no effect for mbtr-1 in a lin-61 or an $h p l-2$ mutant background. 
$\mathbf{A}$

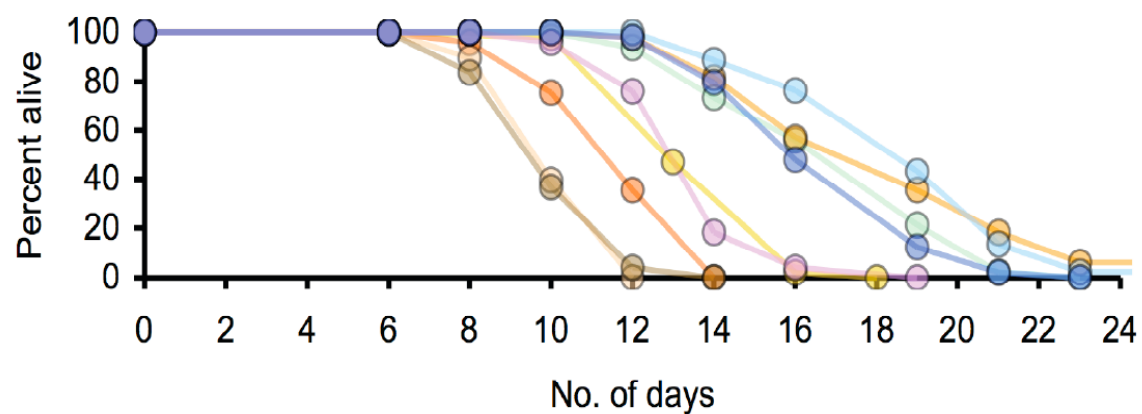

$\mathbf{B}$

Life span $24.5^{\circ} \mathrm{C}$

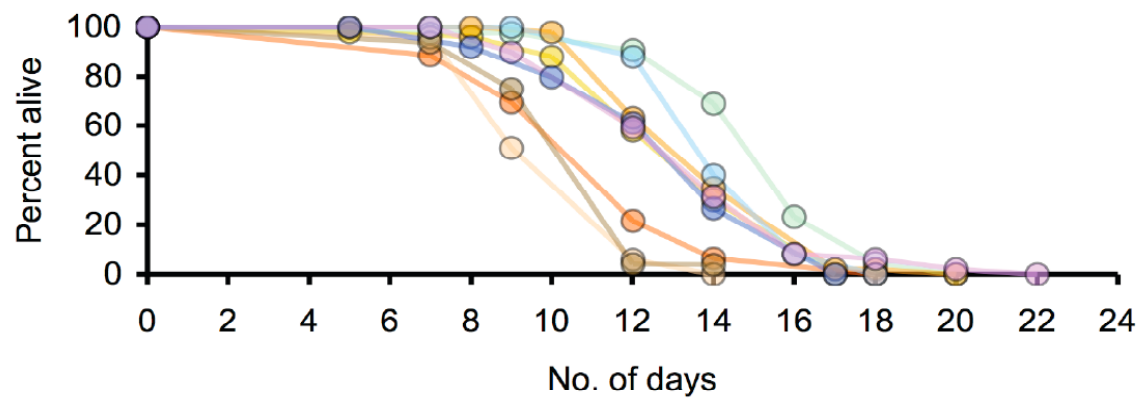

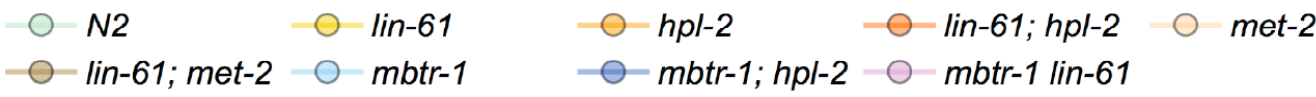

C
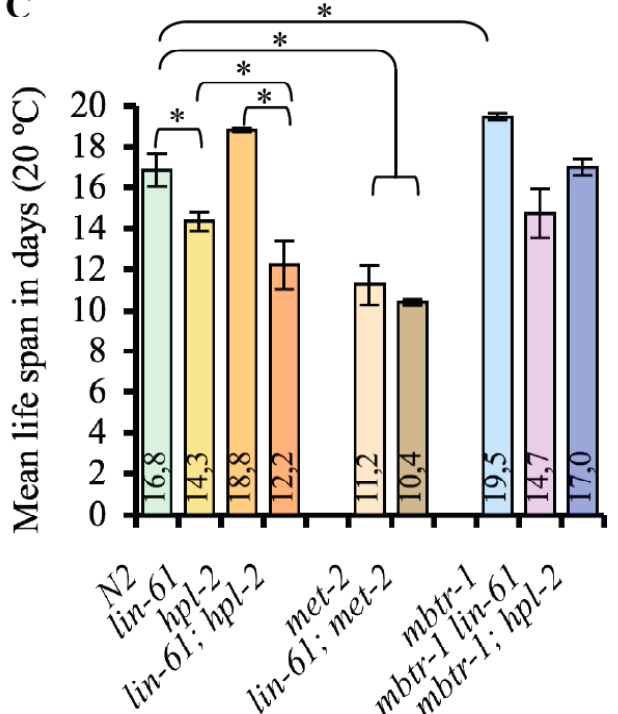

D

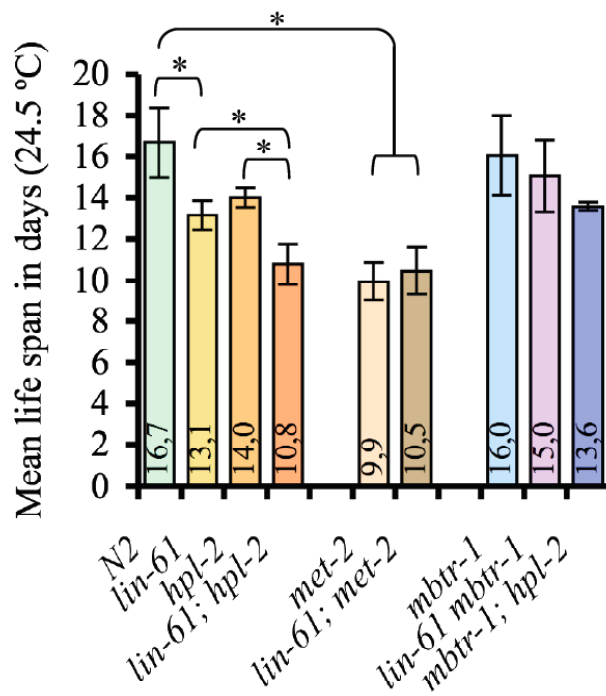

\section{Figure 3-16: lin-61 causes a reduced life span}

(A; B) Representative life span curves of worm populations $(n=50)$ of indicated genotype (see color code in legend below the curves) assayed at $20^{\circ} \mathrm{C}(\mathbf{A})$ or $24.5^{\circ} \mathrm{C}(\mathbf{B})$. The percentage of worms that were alive at each observation day was calculated and plotted. No. of days: number of days from egg (C; D) Mean life span in days of at least two trials per genotype assayed either at $20^{\circ} \mathrm{C}$ (C) or at $24.5^{\circ} \mathrm{C}$ (D) Error bars represent standard deviation; Asterisks and brackets mark strains that differ significantly in life span with a p-value of $<0.05$ ( $p$-values: calculated with a standard Student t-test) 


\subsection{Function of LIN-61 within the synMuvB pathway}

The identification of LIN-61 as a synMuvB protein as well as the synergistic effects of lin-61 loss-of-function with $\mathrm{hpl}-2$ and met-2 loss-of-function support the assumption that LIN-61 exerts biological functions by interaction with the repressive chromatin mark H3K9me3. To study the biological impact of LIN-61 H3K9me3 binding, the role of LIN-61 as synMuvB factor was further analyzed.

\subsubsection{LIN-61 H3K9me3 binding is essential for LIN-61 function within the synMuvB pathway}

\subsubsection{Particular lin-61 alleles that cause a synMuv phenotype in a synMuvA mutant background encode proteins impaired in $\mathrm{H3K9me3}$ binding}

Harrison et al. [100] identified several lin-61 alleles that cause a synMuv phenotype in combination with loss-of-function in synMuvA class genes. Five alleles containing missense mutations in the coding sequence were described: lin-61(n3624) encodes a LIN-61 P123S mutant protein with a conserved proline at the C-terminal end of the first MBT domain exchanged to serine; lin-61(n3736) encodes a LIN-61 F247S mutant protein with a phenylalanine within the second MBT domain exchanged to serine; lin-61(n3807) encodes a LIN-61 G250F mutant protein with a conserved glycine within the second MBT domain exchanged to phenylalanine; lin-61(n3447) encodes a LIN-61 S354N mutant protein with a serine within the third MBT domain exchanged to asparagine and lin-61(n3922) encodes a LIN-61 G445R mutant protein with a glycine within MBT repeat four exchanged to arginine (see Figure 3-3 and [100]). The Muv rates for double mutants of each of the lin-61 missense alleles with the class A synMuv mutation lin-56(n2728) determined at $20^{\circ} \mathrm{C}$ by Harrison et al. [100] are shown in Figure 3-17 A. Some of these mutations seem to disrupt LIN-61 protein stability. Harrison et al. [100] showed in western blot analysis of total worm extract that all strains but lin-61(n3447) have decreased LIN-61 protein level with the levels in lin-61(n3809) and lin-61(n3922) being barely detectable compared to the wild-type LIN-61 level (Figure 3-17 A).

However, the protein level in lin-61(n3447) was not reduced and residual LIN-61 protein was detected in other strains. I hypothesized that these mutations might cause impaired H3K9me3 interaction. To test this hypothesis, corresponding point mutant proteins were in vitro translated (with the TNT system) as MYC-tagged proteins and 
analyzed in peptide pull-down experiments using $\mathrm{H} 3 \mathrm{~K} 9 \mathrm{me} 3$ and $\mathrm{H} 3 \mathrm{~K} 9 \mathrm{me} 0$ peptides

(Figure 3-17 C-G).

A

\begin{tabular}{lc} 
& $\%$ Muv $(n)$ \\
\cline { 2 - 2 } lin-61 allele & With lin-56(n2728) \\
lin-61(n3624) & $85(220)$ \\
lin-61(n3807) & $83(136)$ \\
lin-61 $(n 3736)$ & $80(313)$ \\
lin-61 $(n 3922)$ & $52(167)$ \\
lin-61 $(n 3447)$ & $47(237)$
\end{tabular}

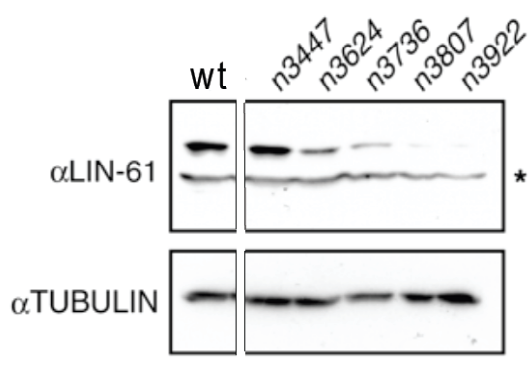

taken from Harrison et al., 2007

B

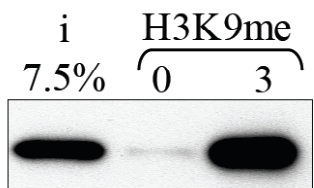

wt

$\mathbf{E}$

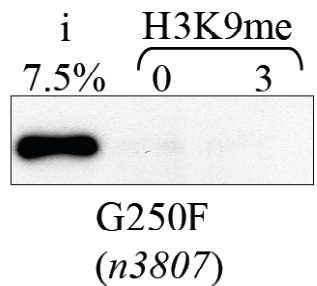

C

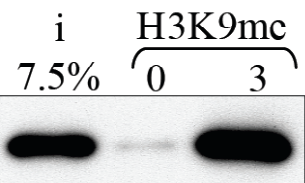

P132S

(n3624)

$\mathbf{F}$

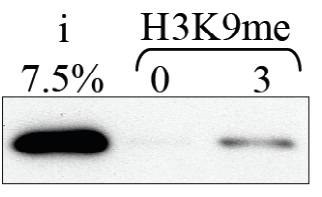

S354N

(n3447)
D

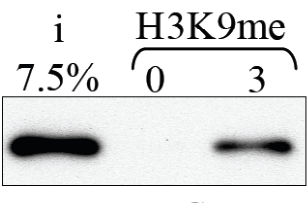

F247S

$(n 3736)$

G

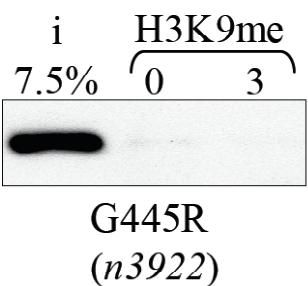

Figure 3-17: LIN-61 point mutants encoded by lin-61 alleles with impaired functions within the synMuvB pathway affect $\mathrm{H3K9me3}$ binding in vitro

(A) Results are taken from Harrison et al. [100]. Left: table shows \% Muv phenotype of indicated genotypes. Right: Western blot analysis using indicated antibodies of protein extract from mixed-stage cultures of indicated genotypes. The asterisk denotes unspecific immunoreactivity (B - G) Peptide pulldown experiments using $\mathrm{H} 3 \mathrm{~K} 9 \mathrm{me} 0$ and $\mathrm{H} 3 \mathrm{~K} 9 \mathrm{me} 3$ peptides were performed with in vitro translated MYC-tagged LIN-61 point mutant proteins corresponding to the LIN-61 protein sequences of lin-61 alleles denoted in (A). Amino acid exchanges compared to wild-type (wt) and corresponding alleles are indicated below the pull-down experiment results. Proteins bound to peptides were eluted by boiling in protein sample buffer, separated by SDS-PAGE and analyzed by western blotting using $\alpha \mathrm{MYC}$ antibody. i: input

The binding affinity of the point mutant proteins towards $\mathrm{H} 3 \mathrm{~K} 9 \mathrm{me} 3$ was evaluated by comparing the $\mathrm{H} 3 \mathrm{~K} 9 \mathrm{me} 3$ bound fraction of the appropriate point mutant with the H3K9me3 bound fraction of wild-type LIN-61 (Figure 3-17 B).

The LIN-61 P132S point mutant protein encoded by lin-61(n3624) interacted with H3K9me3 peptides similar to wild-type LIN-61 (Figure 3-17 C). Noticeably, in $C$. 
elegans extract there was less protein in lin-61(n3624) compared to the wild-type LIN-61 protein level.

LIN-61 F247S point mutant protein encoded by lin-61(n3736) showed strongly reduced interaction with $\mathrm{H} 3 \mathrm{~K} 9 \mathrm{me} 3$ peptide compared to wild-type and no H3K9me3 peptide interaction could be detected for LIN-61 G250F and for LIN-61 G445R mutant protein encoded by lin-61(n3807) and lin-61(n3922), respectively (Figure 3-17 D; E; G). But in these cases reduced protein stability of the corresponding mutant proteins in C. elegans was also detected and could be a reason for an impaired function as synMuvB factor (Figure 3-17 A).

Interestingly, LIN-61 S354N point mutant protein shows strongly diminished interaction with $\mathrm{H} 3 \mathrm{~K} 9 \mathrm{me} 3$ peptide in vitro (Figure 3-17 F).

These results indicate that at least for the lin-61(n3447) allele that was identified in an unbiased screen to cause a synMuv phenotype in a sensitized synMuvA loss-offunction background, impaired methyl mark binding is the likely cause for loss-offunction within the synMuvB pathway, as western blot analysis of worm extract indicates that the mutant protein in stably expressed.

\subsubsection{LIN-61 defective in H3K9me3 binding is not functional in the synMuvB pathway}

To more directly elucidate the significance of the H3K9me3 methyl mark binding capability of LIN-61 with respect to its function within the synMuvB pathway, rescue experiments were performed in the lin-61; lin-15A mutant. To rescue the Muv phenotype of lin-61; lin-15A animals, a DNA fragment from the cosmid R06C7, which contains lin-61 as the only complete predicted open reading frame, was microinjected together with the marker plasmid pFR4 into individual double mutant animals (2.5.15). Seven transgenic lines with the genotype lin-61; lin-15A; Ex[lin61(+) rol-6(su1006)] were established. The seven lin-61; lin-15A; Ex[lin-61(+) rol6(su1006)] lines showed a reduced penetrance of Muv phenotype ranging from 35.8\% to $12.1 \%$ at $24.5^{\circ} \mathrm{C}$ compared to $98.2 \%$ Muv, detected for non-transgenic animals (Figure 3-18 A).

To directly analyze if a LIN-61 protein impaired in H3K9me3 interaction still has the capacity to function in the repression of ectopic vulval cell fate development, a DNA fragment encoding a LIN-61 F452A W455A F459A triple aromatic cage point mutant 
protein, shown to not interact with $\mathrm{H} 3 \mathrm{~K} 9 \mathrm{me} 3$ in peptide pull-down experiments (3.2.3.2) was injected. Four lines were established with the genotype lin-61; lin-15A; Ex[lin-61(triple aromatic cage point mutant) rol-6(su1006)]. None of these four lines showed any rescue of lin-61; lin-15A Muv phenotype (Figure 3-18 A). Importantly, western blot analysis performed on worm lysates that were made from animals of different lines revealed that LIN-61 protein was stably expressed in the analyzed lines (Figure 3-18 B). Therefore, LIN-61 defective in H3K9me3 binding is not functional within the synMuvB pathway.

$\mathbf{A}$
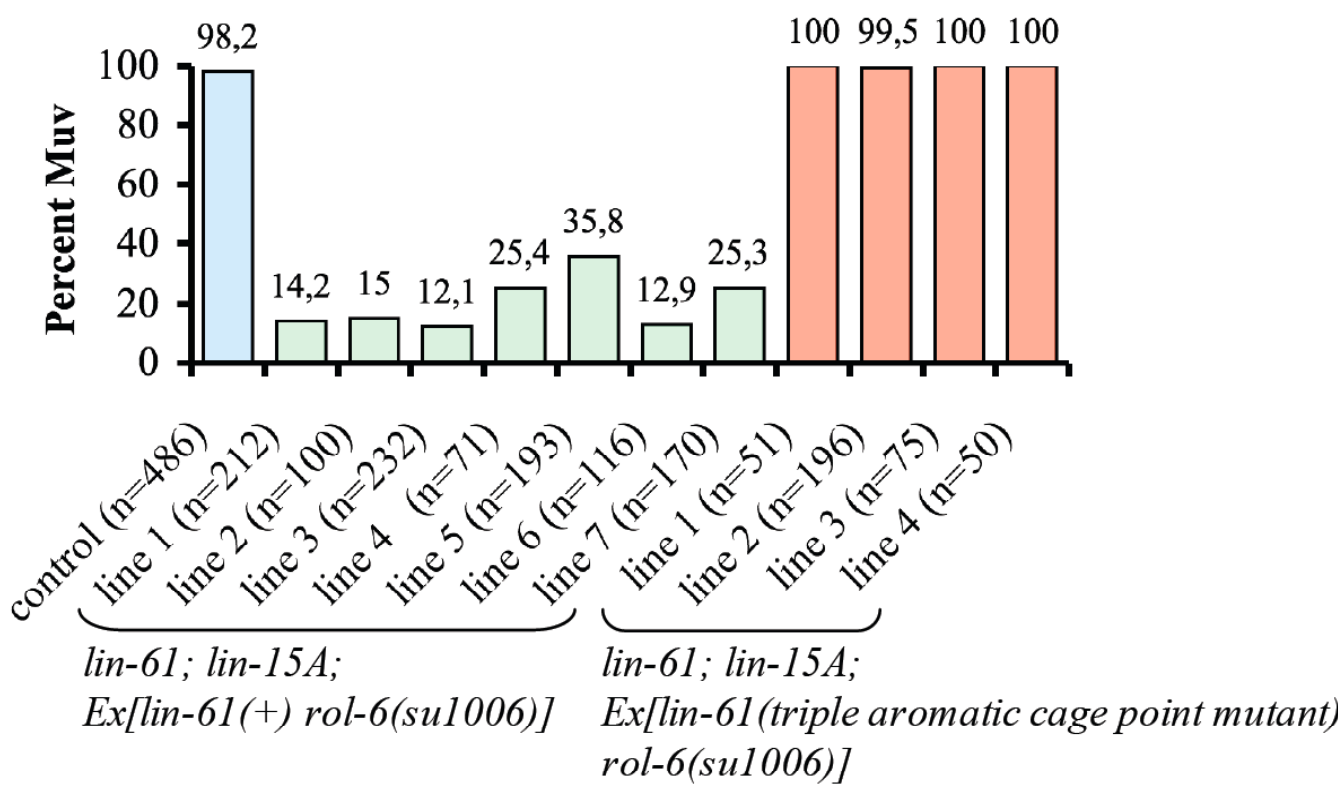

B

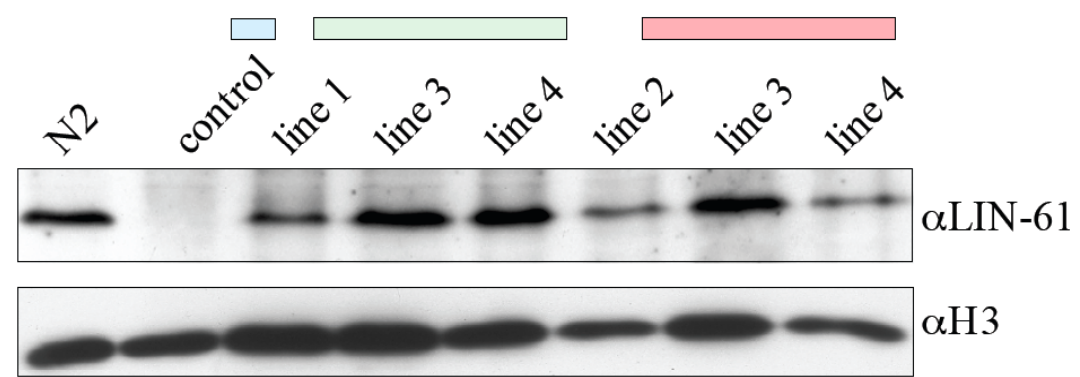

Figure 3-18: The Muv phenotype of lin-61; lin-15A is not rescued by LIN-61 containing a triple aromatic cage point mutation

(A) Rescue of Muv phenotype in lin-61; lin-15A mutant worms. Percent Muv of indicated lines are plotted. lin-61; lin-15A; Ex[lin-61(+) rol-6(su1006)](green bars) indicate lines (1 - 7) generated by microinjection of a genomic $C$. elegans DNA fragment (4387 bp) comprising the lin-61 gene [lin-61 $(+)$ ] into lin-61; lin-15A animals. lin-61; lin-15A; Ex[lin-61(triple aromatic point mutant) rol6(su1006)] (red bars) indicate lines $(1-4)$ generated by microinjection of the same DNA fragment but 
with three point mutations introduced so that the coding lin-61 sequence encodes a LIN-61 F452A W455A F459A triple point mutant protein. The dominant rol-6 (su1006) plasmid pRF4 was co-injected as marker for transgenic animals. Worms were raised at $24.5^{\circ} \mathrm{C}$ and percent Muv was scored using a dissecting microscope. As control (blue bar), worms were scored that have lost the extrachromosomal array. n: number of worms analyzed. (B) Crude C. elegans lysates generated from N2 worms, from control worms (indicated by blue bar), from worms of three lin-61; lin-15A; Ex[lin-61(+) rol6(su1006)] lines (indicated by green bar) and from worms of three lin-61; lin-15A; Ex [lin-61(triple aromatic point mutant) rol-6(su1006)] lines (indicated by red bar) were separated by SDS-PAGE and analyzed by western blotting using $\alpha \mathrm{LIN}-61$ (rabbit) antibodies. $\alpha \mathrm{H} 3$ antibody staining was used as loading control.

\subsection{Identification of LIN-61 interaction partners}

Presumably LIN-61 exerts its functions not solely by interaction with the H3K9me3 repressive chromatin mark but in concert with other proteins. To investigate if LIN-61 is stably associated with other factors, gel filtration chromatography of $\mathrm{N} 2$ C. elegans extract (mixed stage) was performed. Besides a LIN-61 protein peak around the monomeric molecular mass of LIN-61 (56.6 kDa), LIN-61 was detected in fractions corresponding to two higher molecular mass peaks. This result suggests that LIN-61 is assembled with other proteins in stable complexes.

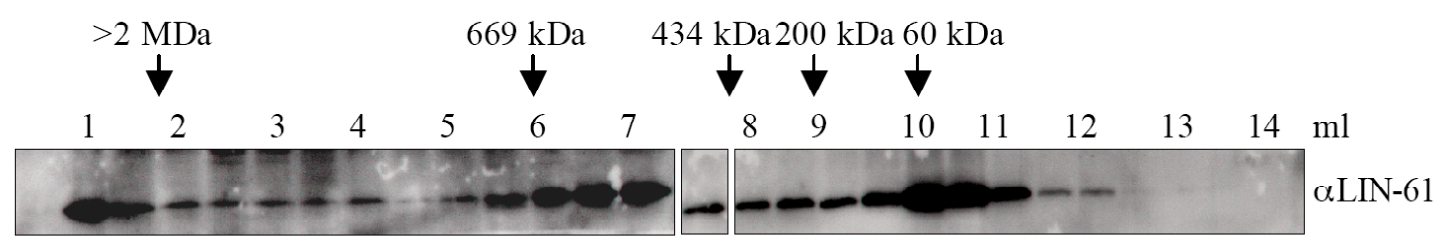

Figure 3-19: Endogenous LIN-61 exists in high molecular weight complexes

C. elegans extract was subjected to Superose 6 gel filtration. Protein fractions were separated by SDSPAGE and analyzed by western blotting using $\alpha$ LIN-61 (guinea pig) antibodies. Elution peaks for protein standards are indicated. The amount of $\mathrm{ml}$ corresponds to the retention volume.

\subsubsection{Identification of LIN-13 as interaction partner of LIN-61}

Harrison et al. [100] performed immunoprecipitation (IP) experiments using $\alpha$ LIN-61 antibodies and analyzed the antibody bound protein fraction for the existence of several proteins of the DRM and NuRD complex in C. elegans by western blotting. None of the tested proteins were identified to interact with LIN-61. I decided to use a more unbiased approach to identify interaction partners of LIN-61. I immunoprecipitated LIN-61 out of C. elegans N2 extract using $\alpha$ LIN-61 (rabbit) antibodies. After washing, recovered proteins were separated by SDS PAGE and 
analyzed by LC-MS/MS. Two separate $\alpha$ LIN-61 immunoprecipitation experiments were performed that were washed in one case (IP a) under mild conditions (100mM $\mathrm{KCl}$ and $0.05 \% \mathrm{NP}-40$ ) and in the other case (IP b) under harsher conditions (300mM $\mathrm{KCl}$ and $0.5 \% \mathrm{NP}-50)$. For control, the same $\alpha \mathrm{LIN}-61$ immunoprecipitation experiments were performed using lin-61(tm2649) protein extract.

In addition, peptide pull-down experiments using $\mathrm{H} 3 \mathrm{~K} 9 \mathrm{me} 0$ and $\mathrm{H} 3 \mathrm{~K} 9 \mathrm{me} 3$ peptides that were performed with N2 C. elegans extract (see 3.1.1) were also performed with lin-61(tm2649) C. elegans extract. In western blot analysis (performed in parallel to LC-MS/MS analysis) LIN-61 was identified in the bound protein fraction of both $\alpha$ LIN-61 immunoprecipitation experiments and the H3K9me3 pull-down out of N2 but not out of lin-61(tm2649) C. elegans extract (Figure 3-20 A).

To extract LIN-61 interacting proteins out of the protein data sets obtained by LCMS/MS for all performed immunoprecipitation and peptide pull-down experiments, the statistical program $\mathrm{R}$ was used. Proteins identified in the $\alpha$ LIN-61 immunoprecipitation experiment out of lin-61(tm2649) C. elegans extract were subtracted from the protein data set obtained for the corresponding immunoprecipitation experiment out of $\mathrm{N} 2$ extract. Proteins specifically present in the H3K9me3 bound protein fraction from N2 extract were obtained by subtracting the $\mathrm{H} 3 \mathrm{~K} 9 \mathrm{me} 0$ bound protein fractions out of N2 and lin-61(tm2649) C. elegans extract and by subtracting the H3K9me3 bound protein fraction out of lin-61(tm2649) $C$. elegans extract.

Besides LIN-61, only two other proteins, LIN-13 and the hypothetical protein B0041.8, were identified as present specifically in all three bound protein fractions of $\alpha$ LIN-61 IP a, $\alpha$ LIN-61 IP $b$ and the H3K9me3 pull-down out of N2 C. elegans extract (but not out of lin-61(tm2649) C. elegans extract) with at least two unique peptides in at least two of the three obtained data sets (see table 6.2.2). The protein sequence of LIN-13 with labeled identified peptides is shown in Figure 3-20 (B). Interestingly, LIN-13 is a synMuvB factor [88, 118]. Also it was shown by Coustham et al. [116] that LIN-13 interacts with HPL-2. The LIN-13 protein sequence (2248 aa) includes the RB binding motif LXCXE, 24 apparent zinc fingers of the $\mathrm{C} 2-\mathrm{H} 2$ class and one apparent zinc finger of the C4 class [118]. 
A

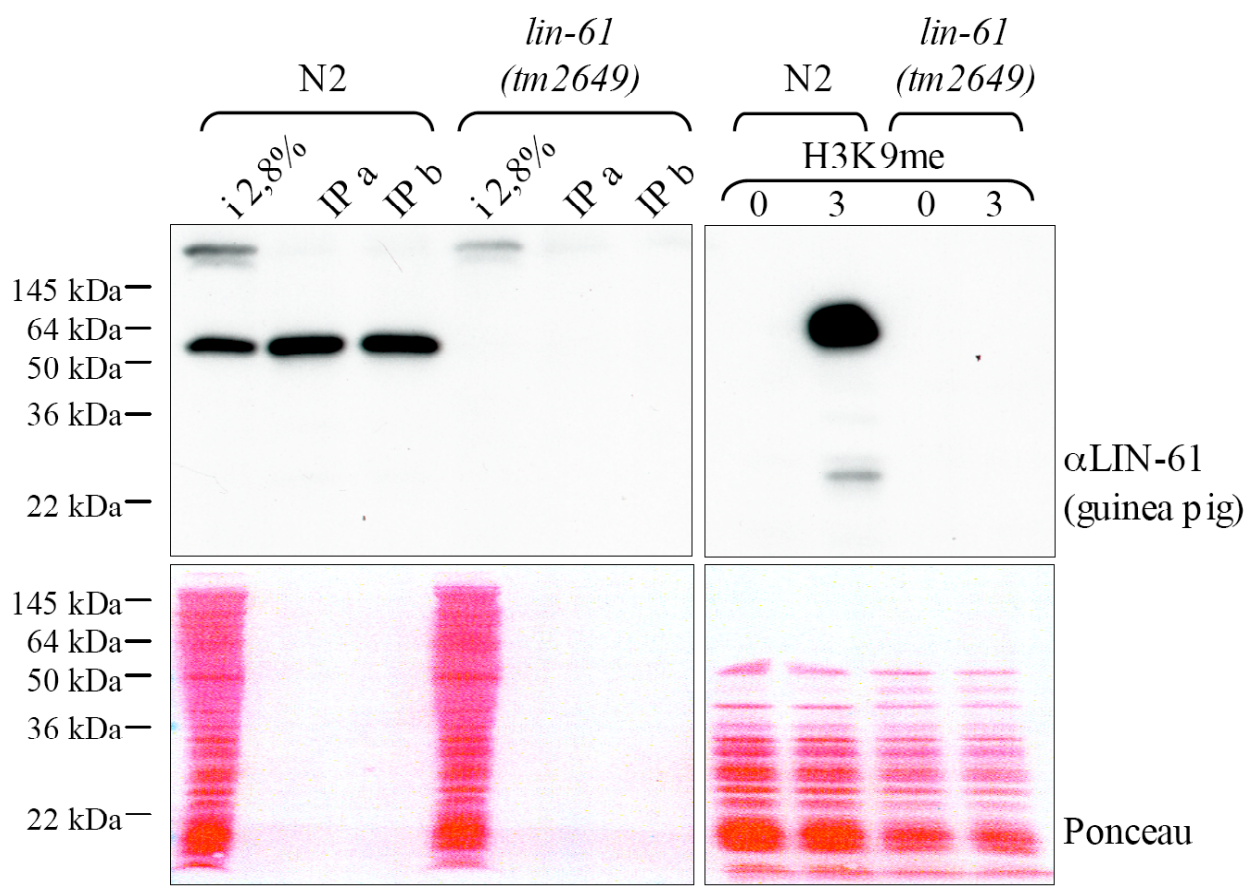

B

Identified protein: LIN-13

MDEFELFQQLNQTAPLVKTEEPEVPDEFQQANNNQSAPLRTGLSDLSHEIAAAKQREEEEAQRLADFMQKDMKEPAVKRKRGSEEYKKDP LESKAPLSTFGHSSRPRRSVNYASIERGDEAQAQSLVTDFGSRGNRKKPKRTRDELDENYMEENEGNSGRKKKPNAKGASRQFQVPGLP TYASQYSRPPKQEDVFK TIVPLAEDAR AEGERVIGFRLDSQPAVRRASGGFRRFCAWLSDNQIFSIMQTVDKLCIVGANNEDHDEILLKSIR HVYNAMPPTFRRDWEYAARKDVFDSRLFVQNMPMPLSEISVTDPRHPPSPIARGTTVRPNCCENQPLFLNMCETIEHYLGHHDVVHLFGCD ICYRVYPSRYELTKHDCKEFAEYLRQLTFKQQTLHLEAAYMYLCCSQCGLWLSVKPSGEGKKGWTYFATALMNHSCQPLVPVVAYFPKP LKDEGKGIRIQFQVMSELNIGLPLSCSECNIEEFHSVVEIEEHFKEKHEANHTCIKCGK TFGTEFMLKHHAQSHTTQTAQFANYLQMSATYQ PPPSSGRLPYVGFGSSIPAIGGLTSGEVOALEASENKKSEFVEPDEYTIRKKLLRWKHAKTKKENRNITDSNEKEFSYEPGESSGEEDFOKSLL EQDNQSSSSSDSDSDSDDFTSSKQKKKRNIKIRGDLGYEHINRNKFFERPESEKEARKRIEKVYKKHVLLSRERLLDPEEALRILEESRMV EQDNQSSSSSDSDSDSDDFISSKQKKKRNIKIRGDLGYEHINRNKFFERPESEKEARKRIEKVYKKHVLLSRERLLDPEEALRILEESRMV HLNSIQSTLADDIAMSCIRTISLPASNCIDPLKDLLLVNKIFYFCTKCNYIFSKDPVVHCLSCEVTEDDLIEVYHAASGPHAGVRCIDPECKAHL CSVISLKTHLSDVHSKQATLELVSGELDNFSENRFDRSLMLMAKHFTQLQFDERTYLARFTDIECFMPFSGLLEAKDQPRPMPIROOPOQT
KPAYSLVRPDIIAPNELMRPYTLTPAIRPGQRIKPYKVPRTSR WYSCSWCDREYESLNQFVDHLTRFHTHPCPSCGKAFSSONTRF THVCSR FAEIKGRGATLCGQCPSCPEIHQVERIFVHMLNRHFSTIEYVLATGELLPPARDVGIRYNHGENGGYGRSYESLK AIEOSVVDPF SPDYRL KQVKISAI PIHGVEI NRI PARDPPMGSFTVCPPKDKNIDPRI MCYMCFI TFDSYDFI THHMDDHPFK WANCPFCAANTPTHFDI QKHI IQ EHVVQISGQACCAFCQEHHRFMSSHILFRCKRVSRCTICGVKSNDPLANRVHIQRSHAL TL RRFOCAYCIKVFVSVGEYYEHECASGGGR

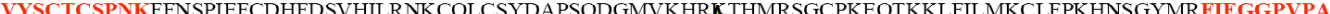
STCT Q ISAGKEKKFKCQKCSLAFY INGSLESHMKDHRQDAGAQLCIEI I YGIPV VIKASWLCKNCCVV FENQPKYQKHMAIHGDICLICIHCSGIA FNHTAIQNHMKSHEEKKVRYSCGTCLCTFASDLALFDHLSVAHGVSLYYFCKVCGFGSTSADSVFQHISIHNGHNYSLVQRFGACPAQLLN YDPTDELEFRSQILNKTIQLVSPSDCSHRSMLLQCETVVSCKTCHCTQAWFNYMAFNNHSEETGFPQFKNVDLANDYRRDFPLSRHLNER NALSMSOFGNAKHGSANHSHGQAQPNKR'IFHEVPYRIAAPRSSLQINGSSMGSVITINGGRVVRPSPPNSMNVILRRAPPQQAPPRRIVI ANSAPNNTNVLRNHVAVTTKCQFKDCDKVLHSEFDRQLHSMHSSNSSWFCRQCGHSPKSEIDLFLHYIQVHLKPAYDKHQSNSFKSNVFHL KCPIRSCTSPEFOSPKAFEKHMRTAHAAELPFEASCCDARFASKALCVKHDOEHASFLDSNGTDASCCPICGSLSMWSLPKDPHTDCLQSHII RHGLDYRSSCRQCLKQFPADVNQDQVIAHILDTHGMSMHGNTFHCNLCTTGTKTVEEFAEHCRKAHVFHILVKSSHSTRGELVVTTGQEY ENYVGLKSVTRASLNSISSQRASNAGETAQPSVLCAGSGNAALLTIAAAIGEPETSNNTAEVLTLD

$\square$ identified in N2_ $\alpha$ LIN-61 IP a

s identified in N2_ $\alpha$ LIN-61 IP b $x$ identified in N2_II3K9me3-PD

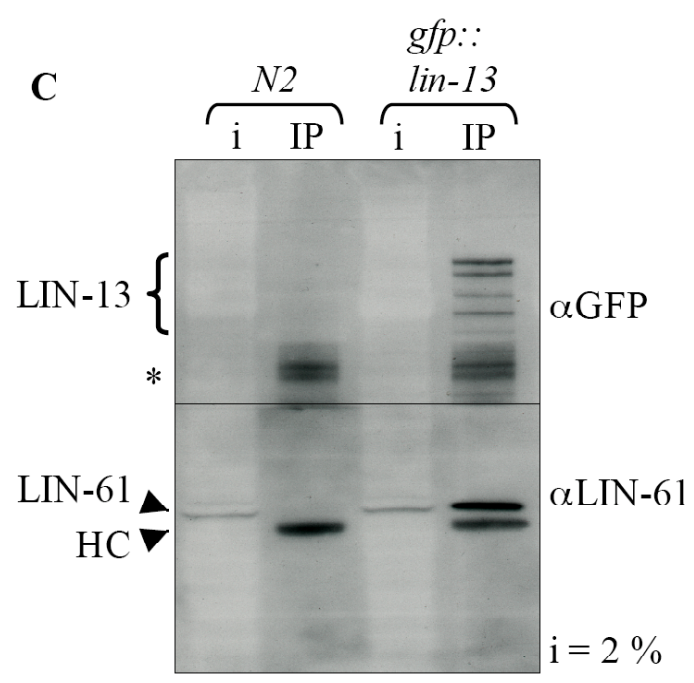




\section{Figure 3-20: Identification of LIN-13 as an interaction partner of LIN-61}

(A) $\alpha$ LIN-61 (rabbit) immunoprecipitation (IP) (IP a: washing steps under mild conditions (100 mM $\mathrm{KCl}$ and $0.05 \% \mathrm{NP}-40$ ); IP b: washing steps under high salt conditions (300 mM KCl and $0.5 \% \mathrm{NP}-$ 40)) and peptide pull-down experiments using $\mathrm{H} 3 \mathrm{~K} 9 \mathrm{me} 0$ and $\mathrm{H} 3 \mathrm{~K} 9 \mathrm{me} 3$ peptides out of wild-type N2 and lin-61(tm2649) C. elegans protein extract. Proteins bound to antibodies or peptides were eluted by boiling and separated by SDS-PAGE. Western blot analysis using $\alpha$ LIN-61 (guinea pig) antibodies and Ponceau staining are shown. LC-MS/MS analysis of eluted protein fractions was performed in parallel. i: input (B) LIN-13 protein sequence, identified in the protein binding fractions of $\alpha$ LIN-61 IP a, $\alpha$ LIN-61 IP $b$ and of H3K9me3 pull-down out wild-type N2 extract. Unique peptides are shown as indicated in the legend below the LIN-13 sequence. (C) $\alpha$ GFP IP out of either wild-type N2 C. elegans protein extract or extract derived from $\operatorname{arIs}[g f p:: l i n-13]$ transgenic animals. Antibody bound protein fractions were separated by SDS-PAGE and analyzed by western blotting using $\alpha$ GFP antibody (top) and $\alpha$ LIN-61 (guinea pig) antibodies (bottom). i: input; HC: antibody heavy chain of antibody; asterisk: nonspecific immunoreactivity of $\alpha \mathrm{GFP}$ antibody on proteins in $\mathrm{N} 2$ and $g f p::$ lin-13 extracts.

To verify the interaction of LIN-13 with LIN-61, a reverse immunoprecipitation experiment was performed. As no specific antibodies are available for LIN-13, protein extract was made from $C$. elegans expressing GFP-tagged LIN-13 (arIs48[gfp::lin-13]). Immunoprecipitation was performed using $\alpha$ GFP antibody. In parallel, $\alpha$ GFP immunoprecipitation experiment was performed out of N2 protein extract as negative control. As shown in Figure 3-20 (C) western blot analysis revealed that LIN-61 was co-immunoprecipitated by $\alpha \mathrm{GFP}$ antibody out of C. elegans extract that contains GFP-tagged LIN-13 but not out of N2 extract. This results confirms direct or indirect interaction of LIN-61 with LIN-13.

The interaction of LIN-61 with B0041.8 was not further investigated or verified in the present study. 


\section{Discussion}

\subsection{C. elegans MBT proteins and HPL-2 interact with H3K9me3}

H3K9me3 peptide pull-down experiments carried out with $C$. elegans protein extract were performed as an unbiased approach to identify chromatin effector proteins in $C$. elegans with functionalities in the readout of the repressive $\mathrm{H} 3 \mathrm{~K} 9 \mathrm{me} 3$ chromatin mark. While $C$. elegans MBT proteins were among the H3K9me3 peptide bound protein fraction, neither of the two C. elegans HP1 homologues, HPL-1 or HPL-2, were identified in this protein fraction although HP1 homologues of other species were shown to generally interact with $\mathrm{H} 3 \mathrm{~K} 9 \mathrm{me} 3$ peptides. This result might indicate that $C$. elegans HP1 proteins do not interact with H3K9me3. Although peptide pulldown experiments followed by MS/MS identification of peptide bound proteins are sensitive and unbiased, only proteins that are found in the pull-down input extract in sufficient amounts can be identified by this approach as putative methyl mark binding proteins. As many chromatin factors that are bound to histones are not solubilized at low salt conditions during extract preparation [131], it is possible that the abundance of some $\mathrm{H} 3 \mathrm{~K} 9 \mathrm{me} 3$ binding proteins within the $C$. elegans extract prepared for this experiment (Cheseman protocol (2.5.8.1), $100 \mathrm{mM} \mathrm{KCl}$ in extract buffer) was not high enough to be identified in the peptide bound protein fraction.

Indeed, a specific interaction of HPL-2 with H3K9me3 peptides was identified in pull-down experiments from $C$. elegans extract that was prepared using $420 \mathrm{mM} \mathrm{KCl}$ for nuclear protein extraction ([131], 2.5.8.2) (Figure 3-14 A), a result that was also obtained by Wirth et al. [117] while the present study was ongoing. These results indicate that HPL-2 is indeed a bonafide $\mathrm{H} 3 \mathrm{~K} 9 \mathrm{me} 3$ interacting protein in C. elegans.

\subsection{MBT proteins in C. elegans define a new class of MBT proteins}

\subsubsection{LIN-61 and MBTR-1 are special MBT proteins}

LIN-61 and MBTR-1, the only two known MBT proteins in C. elegans, were identified as $\mathrm{H} 3 \mathrm{~K} 9 \mathrm{me} 3$ peptide binding proteins out of $C$. elegans extract (Figure 3-1). For LIN-61 I showed that the interaction of LIN-61 with a H3K9me3 peptide is direct and not mediated by other factors (Figure 3-4). In addition, expanded peptide pull-down experiments using several different histone tail peptides with endogenous 
and with recombinant purified LIN-61 revealed a sequence and methylation state specific binding of LIN-61 towards higher methylated (me2/me3) H3K9 peptides (Figure 3-5; Figure 3-6).

Although I could not yet express recombinant protein and analyze its interaction with different histone modifications directly, it is likely that MBTR-1 has similar binding characteristics compared to LIN-61 as in vitro translated MYC-tagged MBTR-1 made in reticulocyte lysate interacted with $\mathrm{H} 3 \mathrm{~K} 9 \mathrm{me} 3$ peptides (Figure 3-2). In addition, in vitro translated MYC-tagged MBTR-1 was bound with higher affinity to a H3K9me3 peptide than to a $\mathrm{H} 3 \mathrm{~K} 9$ mel peptide in pull-down experiments (data not shown).

These results are in stark contrast to the binding characteristics of all other studied MBT proteins (1.2.1.1) indicating that MBT proteins in C. elegans might have evolved differently compared to MBT proteins in other species. As only a subset of MBT repeats of Drosophila (SCM and dSFMBT) and human (SCML2, L3MBTL1, L3MBTL2, MBTD1) MBT proteins has been analyzed in detail regarding methyl mark interaction, it is possible that other MBT proteins possess binding characteristics similar to the C. elegans MBT proteins.

The first obvious difference of LIN-61 compared to other investigated MBT repeats is that LIN-61 shows sequence specific binding towards the surrounding methyl lysine mark sequence, which links this protein to cellular mechanisms involved in the readout of the $\mathrm{H} 3 \mathrm{~K} 9$ methyl mark.

In contrast, the promiscuous interactions shown in vitro with diverse mono- or dimethylated lysines (H3K4me1/me2, H3K9me1/2, H3K36me1/me2, H3K27me1/me2, H4K20me1/me2, H1K26me1/me2) irrespective of sequence environment of other MBT repeats in higher eukaryotes are still an enigma. It is not understood how such binding behavior could be translated into specific biological functions. It is certainly still unclear whether endogenous MBT proteins when posttranslationally modified and/or in the context of other factors/complexes also show promiscuity in methyl mark binding, as all analyses so far have been carried out with recombinant MBT protein preparations. Since several MBT proteins are implicated in gene repression (1.2.2), it seems unlikely that an interaction with $\mathrm{H} 3 \mathrm{~K} 4 \mathrm{me} 1$, a methyl mark consistently associated with active chromatin, is of biological relevance for these proteins. Endogenous MBT proteins in Drosophila or human cells might accomplish sequence specific recognition of methyl marks via interaction with other factors needed to obtain sequence specificity. In this sense, it has been proposed that 
L3MBTL1 might specifically interact with H4K20me1 in vivo since it was shown to interact with PR-SET7, a H4K20me1 specific HMT, and given the observation that knock down of PR-SET7 in K562 cells decreased chromatin association of L3MBTL1 [58].

\subsubsection{LIN-61 selectively binds to lysine in higher methylation state}

The second unique binding characteristic of $C$. elegans MBT proteins identified in my studies is the methyl state preference for higher methylated lysine residues. This observation is in contrast to other investigated MBT proteins that discriminate against tri-methylated lysine (1.2.1.1). This finding implicates differences within the methyl lysine binding pockets of $C$. elegans MBT proteins compared to other MBT repeats for which different crystal structures showed corresponding architecture [49, 55-57, 59].

These results indicate that a simple classification of chromatin effector protein reader modules into general binding modules that specifically interact with PTMs cannot be undertaken based on sequence homologies alone.

Indeed, all chromo domains with a complete set of conserved aromatic cage residues show methyl state specific interaction with di- and tri- methylated lysine [48, 52, 145 , 146] but some chromo domains lacking a complete set of aromatic cage residues [48] were identified to interact instead with DNA (dMi-2, [147]) or RNA (MOF, [148]).

In addition, methyl state specific differences could be identified for double tudor domains of the JMJD2A demethylase, which specifically interact with H3K4me3 [149] and the double tudor domains of 53BP1, which preferentially interact with H4K20me2 and H4K20me1 but not with H4K20me3 peptides in ITC measurements [150]. Apart from being composed of two tudor domains with significant sequence similarity, these tudor domains adopt different fold topologies whereby in case of 53BP1 the two tudor domains form independently folded domains and in case of JMJD2A the two tudor domains are highly interconnected. Further, the methyl lysine binding pockets of both proteins are differently arranged within the double tudor domains $[42,149,150]$.

Unfortunately, no crystal structure for either of the two C. elegans MBT proteins is available. Therefore, I used sequence alignment and structure function analysis to deduce whether the overall fold of the four MBT repeats of LIN-61 might be similarly arranged as in other four MBT repeat proteins. Crystal structures of dSFMBT, 
L3MBTL2 and MBTD1 show that the first MBT repeat sticks out of a highly interdigitated propeller like structure formed by the last three MBT repeats in these four MBT repeat proteins. Nonetheless, the N-terminal arm of the first MBT domain interacts with the core domain of the fourth MBT repeat (1.2.1.3).

I showed that for LIN-61 the first MBT domain is not necessary for the interaction with H3K9me3 peptide (Figure 3-7 A). This result indicates either that in LIN-61 the four MBT repeats are differently arranged as compared to other four MBT repeat proteins or that the first of the four MBT repeats in general is not required for methyl lysine binding. This has not been analyzed for other MBT proteins before. Although crystal structures show that the first MBT domain interacts with MBT domains two and four, one possible explanation might be that in case the first MBT domain is deleted, the N-terminal arm of the second MBT domain that would normally interact with the first MBT core domain can substitute the interaction of the N-terminal arm of the first MBT domain with the core domain of MBT repeat four.

On the other hand it is conspicuous that the sequence alignment of LIN-61 and MBTR-1 with the four MBT repeats of other MBT proteins shows stretches of extra residues for LIN-61 and MBTR-1 within MBT domain two that might account for structural differences between the four MBT repeats of $C$. elegans MBT proteins compared to the other four MBT repeats (Figure 3-3).

The finding that all three MBT domains two to four are required for H3K9me3 binding (Figure 3-7) leads to the suggestion that the overall fold of these MBT repeats has an important impact on methyl mark binding. To solve this issue in detail, a crystal structure of the MBT repeats of LIN-61 will be needed.

In contrast to complete distinct binding pockets for JMJD2A (composed of two interconnected tudor domains) and 53BP1 (composed of two separate tudor domains) I could show by sequence alignment and functional analysis that the only methyl lysine binding pocket of LIN-61 is found in the fourth MBT repeat as it is in other MBT proteins containing four repeats (Figure 3-8; [56, 57, 63]).

In structure function analysis I revealed that only two of the three conserved aromatic residues shown to be essential for methyl lysine interaction in L3MBTL1 [59] are essential for H3K9me3 binding of LIN-61 in peptide pull-down experiments (Figure 3-8 D, F, G). This finding indicates that residues shown to participate in forming the methyl-lysine binding pocket in other MBT proteins are essential for LIN-61. 
However the hydrophobic cage for methyl lysine interaction in LIN-61 obviously is composed differently as compared to other MBT proteins.

The general assumption is that the aromatic cage residues in the binding pockets of proteins that selectively interact with lower methylated lysine $(\mathrm{Kmel} / \mathrm{Kme} 1)$ serves besides stabilization of methyl-lysine interactions as size selective filter (1.2.1.2, [42, 150, 151]). Since LIN-61 preferentially binds to tri-methylated lysine it is apparent that in LIN-61 a modified aromatic cage is formed as compared to other MBT proteins.

According to the sequence alignment in Figure 3-3, a conserved leucine that is shown to participate along with the three conserved aromatic cage residues in the formation of the hydrophobic methyl-lysine binding cage in L3MBTL1 and L3MBTL2 [57, 59] is replaced by a tryptophan in the MBTR-1 sequence and by a phenylalanine in the LIN-61 sequence. I hypothesize that in case of LIN-61 F434 instead of F452 might be involved in the formation of an aromatic cage.

Another inconsistence for methyl lysine binding of LIN-61 compared to other MBT proteins is that the mutation of a conserved aspartate to alanine (D429A) in LIN-61 caused only diminished binding to the H3K9me3 peptide (Figure 3-8 C) but did not abrogate methyl-lysine interaction as shown for other MBT proteins (1.2.1.2). While in other MBT proteins, the primary function of the conserved aspartate is to stabilize the binding of mono- and di-methyl lysine by forming a hydrogen bond with a methyl-ammonium proton, this function cannot be valid for the interaction with trimethylated lysine, as the tri-methyl-ammonium group cannot serve as hydrogen donor.

Overall, the results indicate that methyl mark binding of LIN-61 takes place in the same MBT domain as in other MBT proteins and that some conserved residues implicated in methyl mark interaction in other MBT proteins hold this function in LIN-61 whereas clear differences could be detected for other conserved residues. However, to better understand the binding mechanism of LIN-61 to H3K9me3 in more detail and in comparison to the selective mono- and di-methyl lysine binding mechanism of other MBT proteins, further structural analysis is required. In addition to providing a better understanding of the methyl lysine binding pocket of LIN-61, a three dimensional structure of the LIN-61 MBT repeats in complex with H3K9me3 peptides would also provide insight how the sequence specificity of LIN-61 toward the H3K9 sequence backbone is accomplished. 


\subsection{Biological functions of $C$. elegans MBT proteins}

In contrast to other MBT proteins in Drosophila or human cells that interact with mono- and dimethylated lysine marks, LIN-61 shows a clear binding preference towards the higher methylated H3K9me state (H3K9me2/me3). Therefore, it is questionable whether LIN-61 possesses functions that can be attributed to MBT proteins in general. But if the interaction with a particular histone PTM is a mechanism to target chromatin effector proteins to a particular genomic region, it might be valid that $C$. elegans MBT proteins share general functions with other MBT proteins. Therefore, mechanisms by which the MBT repeats of $C$. elegans proteins exert their biological functions could be translated to some extent to functional mechanisms of MBT repeats in other proteins. Supporting this argument, I showed an impact on lin-3 repression for LIN-61 (Figure 3-13). Indeed, several MBT proteins in Drosophila and in human cells possess functions in gene repression (1.2.2.1, 1.2.2.2). Therefore the dissection of biological functions and working mechanisms of $C$. elegans MBT proteins will contribute to a better understanding of the working mechanisms of MBT repeats in general. Interestingly, the two C. elegans MBT proteins are the only known MBT proteins that do not contain any other recognizable domains apart from MBT repeats. Thus, the functions of LIN-61 are probably all mediated by the MBT repeats.

\subsubsection{LIN-61 within the synMuvB pathway}

Here, lin-61 was identified as a synMuvB gene and I showed that LIN-61 exerted a repressive function on lin-3 expression in this pathway (Figure 3-13).

\subsubsection{LIN-61 acts within the synMuvB pathway via H3K9 methyl mark binding}

In two different approaches I showed that H3K9 methyl mark interaction is an essential function of LIN-61 to exert its functions as a synMuvB factor in repression of ectopic vulval cell fate development.

In the first approach, rescue experiments were performed in lin-61; lin-15A mutant $C$. elegans by microinjection (Figure 3-18). All seven lines, generated by microinjection of a genomic DNA fragment comprising the lin-61 gene, showed robust rescue of the lin-61; lin-15A Muv phenotype. In contrast, all four lines generated by microinjection 
of a DNA fragment encoding a LIN-61 F452A W455A F459A triple point mutant did not rescue the Muv phenotype. Microinjection experiments have the drawback that large extrachromosomal arrays are formed and therefore lin-61 gene expression from these arrays might not reflect the endogenous lin-61 gene expression levels in all developmental stages and in all cells. Nevertheless, western blot analysis of worm lysates generated from mixed stage worms of several generated lines indicate that LIN-61 is stably expressed in all tested lines in comparable amounts. In addition, the fact that at least four independent lines were analyzed with consistent results supports the finding that in contrast to wild-type LIN-61, LIN-61 F452A W455A F459A is not functional in the synMuvB pathway. As the LIN-61 F452A W455A F459A mutant was shown in vitro to not interact with $\mathrm{H} 3 \mathrm{~K} 9 \mathrm{me} 3$ peptides, these results indicate that H3K9me3 methyl mark interaction is an essential function of LIN-61 within the synMuvB pathway.

In a second approach I investigated the capacity of in vitro translated LIN-61 point mutant proteins to interact with $\mathrm{H} 3 \mathrm{~K} 9 \mathrm{me} 3$ peptide. The LIN-61 point mutants correspond to proteins encoded by lin-61 missense alleles that were identified in unbiased screens as synMuv causing alleles in a synMuvA loss-of-function background. Out of these point mutant proteins I could identify one missense mutation (LIN-61 S354N) that showed strongly diminished interaction with H3K9me3 peptide in vitro but was shown to be stably expressed in vivo (Figure 3-17 F, [100]). This result clearly indicates together with the rescue experiment that $\mathrm{H} 3 \mathrm{~K} 9 \mathrm{me} 3$ binding is essential for LIN-61 to act as a synMuvB protein.

Interestingly, the point mutation of LIN-61 encoded by lin-61(n3447) is not located in the peptide binding MBT repeat four but in the core domain of MBT repeat three. This finding further supports the assumption that MBT two to four of LIN-61 are forming one unit with high interdigitation between the single MBT domains and that all three repeats are necessary for methyl mark binding (3.2.3.1). As the protein is stably expressed in C. elegans, it is unlikely that this point mutation causes severe missfolding of the protein. It would be highly interesting to dissect the impact of the amino acid exchange S354N on H3K9me3 interaction of LIN-61 by solving corresponding crystal structures.

In vitro translated LIN-61 P132S did not cause any reduction in H3K9me3 peptide binding affinity. P132 is located in the C-terminal end of MBT core domain one. Therefore the result is consistent with the finding that MBT one of LIN-61 is not 
essential for methyl mark binding in vitro (3.2.3.1). Western blot analysis of Harrison et al. [100] reveals that the P123S mutation disrupts protein stability of endogenous LIN-61 encoded by lin-61(n3624). A strongly reduced LIN-61 protein level is an obvious explanation for the synMuv phenotype that is caused by lin-61(n3624) in a synMuvA mutant background. However, due to the fact that about half the amount of LIN-61 could still be detected in worm lysate made from lin-61(n3627) as compared to LIN-61 levels detected in lysate made from wild-type worms (Figure 3-17 C, [100]), other functions of LIN-61 apart from the H3K9me3 binding capacity might be impaired by this mutation. LIN-61 levels in $n 3736, n 3807$ and $n 3922$ are severely reduced, which is likely the major reason for the synMuv phenotype in combination with the loss-of-function allele lin-56(n2728). Nevertheless the finding that the mutation F247S diminished and that the mutations G250F and G445R abolished binding of LIN-61 to H3K9me3 peptide, leads to the suggestion that residual LIN-61 protein levels in lin-61(n3736), lin-61(n3807) or lin-61(n3922) are not functional in the synMuvB pathway.

\subsubsection{LIN-61 interacts with the synMuvB factor LIN-13}

I showed that LIN-13 interacts directly or indirectly with LIN-61 (3.5.1). Interestingly LIN-13 is a synMuvB class protein [118] and in addition it was reported that it interacts with HPL-2 [116]. As HPL-2 was not identified by MS/MS analysis in the H3K9me3 pull-down experiments in which LIN-13 was identified and due to the fact that LIN-13 was not identified in the $\mathrm{H} 3 \mathrm{~K} 9 \mathrm{me} 3$ peptide bound protein fraction out of lin-61 mutant C. elegans protein extract, H3K9me3 interaction of LIN-13 is likely mediated by LIN-61. In addition, a LIN-61/LIN-13 interaction was identified in a reciprocal immunoprecipitation experiment.

LIN-13 is an essential protein in C. elegans but its function is unknown. LIN-13 contains 25 zinc fingers that might have functions in protein-DNA or protein-protein interaction. Also, an RB protein-binding motif is present. Therefore one functional aspect of LIN-13 in the synMuvB pathway might be to serve as an interaction platform coordinating the interplay of other synMuvB factors.

Interestingly, several MBT proteins in other organisms are functionally linked to a number of proteins homologous to $C$. elegans synMuvB factors. It has been shown that L3MBTL1 promotes repression of E2F (EFL-1) target genes and that it is 
associated with RB (LIN-35), HP1 $\gamma$ (HPL-2) and RPD3 (HDA-1) (1.2.2.1). In addition dSFMBT was shown to be involved together with Mi-2 (LET-418) and MEP-1 in SUMO (SMO-1) mediated transcriptional repression (1.2.2.2, [76]). Therefore one hypothesis is that LIN-61 might exert its repressive function within the synMuvB pathway in a concerted manner together with one or more of these factors. However, none of these factors were identified in $\alpha$ LIN-61 immunoprecipitation experiments performed in the present study and neither HDA-1 nor HPL-2 was identified in $\alpha$ LIN-61 immunoprecipitation experiments performed by Harrison et al. [100]. Nonetheless these negative results do not preclude an interaction, as the experiments were either performed using $C$. elegans extract from mixed stage worms or from embryos and might therefore not reflect protein interactions that take place during vulva development (L3 stage).

\subsubsection{3 lin-61 genetically interacts with $\mathrm{hpl}-2$ and met-2}

lin-61; met-2 cause a low penetrant synMuv phenotype at $24.5^{\circ} \mathrm{C}$ (Table $\left.3-2\right)$.

This result is in line with the observation that in a synMuvA mutant background both lin-61 and met-2 cause elevated lin-3 mRNA levels in L3 stage worms (Figure 3-13, $[112,119])$, but that the lin-3 mRNA level in a triple lin-61(n3809); met-2(n4256); lin-15A(n433) mutant background is even more enhanced [119]. This result leads to the hypothesis that in lin-61; met-2 L3 stage worms the lin-3 mRNA level might as well be elevated compared to the corresponding single mutants. De-repression of lin-3 in lin-61; met-2 mutant worms might reach a lin-3 threshold level prone to induce ectopic vulval cell fate development in a low percentage of worms even in the absence of a synMuvA gene mutation. These findings indicate partly redundant functions of LIN-61 and MET-2, sufficient to adequately suppress ectopic vulval cell fate development in corresponding single mutant worms.

met-2 encodes a H3K9 HMT and it was shown that the enzymatic activity of MET-2 is important for its function in the synMuvB pathway [112]. Together with my finding that $\mathrm{H} 3 \mathrm{~K} 9 \mathrm{me} 3$ binding is an essential function of LIN-61 within the synMuvB pathway (3.4), this result might indicate that both proteins act by placing and respectively interacting with the $\mathrm{H} 3 \mathrm{~K} 9 \mathrm{me} 3$ mark in a chromatin mediated mechanism within the synMuvB pathway. In case of MET-2 acting upstream of LIN-61, no genetic interaction between lin-61 and met-2 would be expected as long as both mutations are null. One explanation for a synthetic Muv phenotype in lin-61; met-2 is 
that met-2 loss-of-function causes no complete abrogation of the H3K9me3 mark in chromatin regions supposed to be regulated by LIN-61. In line with this argument, it has been shown that next to a strong reduction of the H3K36me3 methyl mark, the level of $\mathrm{H} 3 \mathrm{~K} 9 \mathrm{me} 3$ is also reduced in protein extract of met-1 mutant embryos (1.3.1.3, [112]). In addition, there are two other putative H3K9 HMTs identified in $C$. elegans, set-11 and set-23. Mutation of set-11 causes no phenotype as single mutant and is not synMuvB. The mutation of set-23 causes an embryonic lethal phenotype and therefore it cannot be excluded that set-23 possess functions as a synMuvB factor [112].

Residual H3K9me3 marks might be sufficient to target a sufficient amount of LIN-61 to corresponding chromatin regions. In addition, H3K9me3 marks, placed by MET-2, can be recognized and bound by other chromatin effector proteins apart from LIN-61 that have functions as synMuvB factor (e.g. HPL-2). These assumptions might explain why both proteins can exert their functions in the synMuvB pathway to some extent independently from each other, although MET-2 is thought to act upstream of LIN-61. Loss-of-function of both factors might severely impair corresponding repressive chromatin states compared to the single mutations. This enhancement might coincide with more pronounced de-repression of lin-3 caused by lin-61; met-2 and as a consequence might result in a Muv phenotype.

Besides genetic interaction with met-2, I showed that lin-61; $h p l-2$ causes a strong synergistic Muv phenotype at $24.5^{\circ} \mathrm{C}$ indicating that LIN-61 and HPL-2 possess redundant functions in repression of ectoptic vulval cell fate decision.

As defects in $\mathrm{H} 3 \mathrm{~K} 9 \mathrm{me} 3$ binding abrogates the function of LIN-61 as synMuvB factor (3.4) and as HPL-2 was identified to interact with H3K9me3 in peptide pull-down experiments (Figure 3-14 A, [117]), both proteins might possess similar H3K9me3 dependent functions in the formation of repressive chromatin states and by that cause gene repression. It was also shown that $h p l-2$ causes enhanced lin-3 expression in a lin-15A mutant background in L3 worms [115]. One possible explanation for the strong synMuv phenotype of lin-61; $h p l-2$ in the absence of a synMuvA mutation is that lin-3 desilencing caused by lin-61; hpl-2 is above a threshold level, as suggested for lin-61; met-2 (see above).

Interestingly, it was also reported that $h p l-2$ loss-of-function causes a synthetic Muv phenotype in a met-2 mutant background [112]. On the one hand similar assumptions can be made to explain independency of both factors in inhibiting expression of 
ectopic vulval cell fates as for LIN-61 and MET-2 (see above). On the other hand it is worth noticing that the genetic interaction of met-2 and hpl-2 in met-2 hpl-2 (87\% Muv at $20^{\circ} \mathrm{C}$ ) [112] is much stronger when compared to lin-61; met-2. Therefore, HPL-2, in contrast to LIN-61, might act to some extent or even exclusively independent from the H3K9me3 methyl mark within the synMuvB pathway, despite its interaction with $\mathrm{H} 3 \mathrm{~K} 9 \mathrm{me} 3$ in pull-down experiments. Supporting this idea is a report showing that HPL-2 localization in nuclear foci of embryo nuclei is mediated by LIN-13 but distinct from H3K9me3 [116].

Reports, showing that $h p l-2$ causes a synMuv phenotype of varying degree in conjunction with several more synMuvB loss-of-function mutations and at $25^{\circ} \mathrm{C}$ already as single mutant $(1.3 .1 .3,[112-114,116])$ complicate the understanding of how HPL-2 might act within the synMuvB pathway and how its function correlates with the function of LIN-61.

In general, as indicated in 1.3.1.3, it becomes more evident that several synMuvB loss-of-function mutations not only cause a synMuv phenotype in combination with synMuvA loss-of-function mutations but in addition with a subset of synMuvB lossof-function mutations. Additionally, most synMuvB double mutation combinations cause a more pronounced synMuv phenotype in a synMuvA mutant background as do single synMuvB mutations. One deduction from these observations is that most synMuvB factors act independently from each other [119]. On the other hand most synMuvB genes encode factors implicated in chromatin regulation. As outlined for LIN-61 and MET-2, the corresponding factors might act in a concerted manner on chromatin despite their genetic interaction. Therefore, to better understand to what extent synMuv factors act redundantly or in a concerted way and how individual factors generally act to achieve repression of ectopic vulval cell fate development, a combination of genetic and biochemical approaches should be used for further dissection of this pathway. The biochemical identification of the DRM complex (1.3.1.3) and the finding that the enzymatic function of MET-2 and the H3K9me3 methyl mark binding capacity of LIN-61 both are essential functions within the synMuvB pathway are starting points to better understand the mechanisms of the synMuvB protein network in repression of ectopic vulval cell fate development. I am confident that these types of experiments will help to better understand chromatin regulation. Besides detailed analysis of synMuvB factors, investigation of the not 
well-characterized group of synMuvA factors will be of great importance to better understand the molecular mechanisms of ectopic vulva cell fate repression.

\subsubsection{LIN-61 possess unique and redundant functions with HPL-2 and MET-2 besides the SynMuvB pathway}

In addition to having a role in controlling vulva cell fate development, I demonstrated that LIN-61 has more general functions in C. elegans. Although I could not detect apparent defects in lin-61 single mutant worms (Figure 3-11 B), I showed that lin-61 causes on average $30 \%$ reduced mean brood size at $24.5^{\circ} \mathrm{C}$ and a reduced mean life span compared to wild-type (Figure 3-15, Figure 3-16). Overall, the defects caused by lin-61 are not severe but they are significant and implicate that LIN-61 is involved in a broader range of processes apart from its function within the synMuvB pathway.

LIN-61 has a repressive function as synMuvB factor and Harrison et al. [100] reported a repressive function of LIN-61 on mat-3 gene expression. Together with the finding that H3K9me3 methyl mark binding is an essential function of LIN-61 in the synMuvB pathway, it is likely that LIN-61 has more general repressive functions on the expression of a broader range of genes. The detected phenotypes are probably caused by mis-regulation of a number of genes, which as a consequence might slightly perturb diverse balanced cellular mechanisms that ensure viability and proper generation of offspring. As the phenotypes detected are not severe, these results indicate that lin-61 causes either only subtle mis-regulation of a number of genes or mis-regulation of a subset of genes that do not have essential functions. To identify genes that are regulated by LIN-61, cDNA microarray gene expression profiles of lin61 mutants in comparison to wild-type and ChIP-on-ChIP experiments for identification of LIN-61 chromatin target sites have to be performed. In addition, LIN-61 might function as a global H3K9me3 methyl mark binding protein in the maintenance of heterochromatin and by this in genome integrity and cellular maintenance.

In contrast to lin-61, hpl-2 causes more severe defects in the generation of offspring. I detected sterility in $13 \%$ of $h p l-2$ worms at $20^{\circ} \mathrm{C}$ and $25 \%$ at $24.5^{\circ} \mathrm{C}$ (Figure $3-15 \mathrm{~A}$ ). These results are in conflict with Schott et al. [114], who reported $0 \%$ sterility at $20^{\circ} \mathrm{C}$ 
but $44 \%$ sterility at $24^{\circ} \mathrm{C}$ and $100 \%$ sterility at $25^{\circ} \mathrm{C}$. Although the exact percentages of sterility are in disagreement, I detected morphological gonadal defects in a subset of $h p l-2$ worms that are consistent with the reported defects of the somatic gonad [114]. In addition, I saw the brood size of $h p l-2$ animals was on average $40 \%$ reduced at $20^{\circ} \mathrm{C}$ and $80 \%$ reduced at $24.5^{\circ} \mathrm{C}$ compared to the mean brood size of wild-type worms (Figure 3-15 B, C). Defects of the gonads probably cause this phenotype as gonadogenesis is a complex process and the development of the somatic gonad and of the germline is coordinated by intercellular signaling [152]. Defects in development of the somatic gonad are prone to cause early germline proliferation defects. In adult hermaphrodites, germline proliferation in the mitotic zone near the DTCs and progression of differentiation from germ cells to oocytes is controlled in tight connection with somatic gonad cells by complex signaling mechanisms.

Gonadal defects are also caused by mutation of the synMuvB gene $h d a-1$ [120]. It has been shown that $h d a-1$ loss-of-function and loss-of-function of several other synMuvB genes including $h p l-2$ causes ectopic expression of lag-2::gfp, which is normally restricted to the DTCs and to vulva cells (1.3.1.4). lag-2 encodes a ligand of the Notch receptor family, which is implicated in somatic gonad cell fate decision and germ cell development [120].

In addition, it was reported that loss-of-function of several synMuvB factors cause ectopic missexpression of $p g l-1$ in somatic cells (1.3.1.4). $p g l-1$ encodes a $\mathrm{P}$ granule component and its expression is normally restricted to the germline.

Overall, these results indicate that several synMuvB factors are involved in germline soma distinction by regulating soma as well as germline restricted gene expression. Defects in germline soma distinction probably have an impact on sterility and reduced brood size and might also cause a reduced life span.

Apart from mild phenotypes in lin-61 single mutants, lin-61 in an hpl-2 mutant background showed strong synergistic effects on sterility and brood-size.

Interestingly, $h p l-2$ loss-of-function, which causes stronger defects on brood size and gonadal development than lin-61, does not cause a reduction in mean life span at $20^{\circ} \mathrm{C}$. hpl-2 loss-of-function nonetheless affects life span in a lin-61 mutant background at $20^{\circ} \mathrm{C}$ and additive effects of these mutants were observed at $24.5^{\circ} \mathrm{C}$ where hpl-2 and lin-61 both reduced C. elegans life span. 
The detected phenotypes indicate unique as well as redundant functions of LIN-61 and HPL-2. The redundancy might be caused either by HPL-2 and LIN-61 controlling different sets of genes that have redundant functions or by regulating common target genes where both factors must be absent to fully de-repress the corresponding genes. In addition, I detected an impact of lin-61 on gonadal defects caused by $h p l-2$. This leads to the suggestion that reduced brood size in lin- 61 single mutants at $24.5^{\circ} \mathrm{C}$ is also caused by (non apparent) gonadal defects. Interestingly Harrison et al. [100] did not detect mis-regulated expression of either $p g l-1$ or lag-2::gfp in lin-61(n3809) C. elegans, indicating that lin-61 associated phenotypes are probably not associated with mis-expression of these genes. In contrast to the results of Harrison et al., Poulin et al. [101] detected ectopic expression of lag-2 caused by lin-61 RNAi but outside gut and epidermis (tissues most affected by lag-2 mis-expression by loss-of-function of other synMuvB factors).

The phenotypic analysis of met-2 mutant worms revealed that met- 2 causes defects in brood size, but only a low level of sterility. Consistent with the assumption that MET2 has impact on gene repression similar to HPL-2 and other synMuvB chromatin regulatory proteins, mis-regulated lag-2::gfp expression was also detected in met-2 animals [112].

Apart from a reduced brood size, met-2 mutant worms showed an apparent reduction in mean life span (Figure 3-16).

lin-61 caused a synergistic reduction in brood-size in a met-2 mutant background at $20^{\circ} \mathrm{C}$ and at $24.5^{\circ} \mathrm{C}$. However, my results on sterility caused by met-2 and lin- 61 ; met2 are inconsistent. At $20^{\circ} \mathrm{C}$ I detected $7 \%$ sterile worms for met-2 but no sterile worms for lin-61; met-2. The overall sterility was significantly increased in lin-61; met-2 as compared to met-2 at $24.5^{\circ} \mathrm{C}$, which is in line with the synergistic effects on brood size caused by lin-61; met-2 at both temperatures. To investigate this inconsistency, the sterility of met-2 and lin-61; met-2 has to be analyzed a second time using a bigger sample size.

The synergistic effect on brood size is not as strong as compared to the effects lin-61 causes in an hpl-2 mutant background. Nonetheless the results indicate that LIN-61 and MET-2 possess to some extent redundant functions. Interestingly the reduced life span detected for met-2 is not further affected by lin- 61 . 


\subsubsection{No biological functions are yet described for MBTR-1}

I did not detect any phenotypic defects in $m b t r-1$ mutant C.elegans. Loss-of-function of mbtr-1 has neither an impact on brood size nor does it cause a reduction in mean life span and MBTR-1 could not be identified as synMuvB factor. In addition, no effects were detected for mbtr-1 in a lin-61 or hpl-2 mutant background. Therefore, although MBTR-1 and LIN-61 both are composed mainly of four MBT repeats and despite the fact that they both interact with $\mathrm{H} 3 \mathrm{~K} 9 \mathrm{me} 3$ peptide, the proteins seem to possess distinct biological functions.

Interestingly, at $20^{\circ} \mathrm{C} \mathrm{mbtr-1}$ worms displayed a slightly enhanced mean life span. Further investigation of MBTR-1 function under this aspect might bring light into the biological function of MBTR-1. 


\section{Summary}

In eukaryotic cells the genome is packaged into chromatin, which is composed of core histones (H2A, H2B, H3, H4), non-histone proteins and DNA.

Packaging of DNA into distinct chromatin states plays a pivotal role not only in the maintenance of intact chromosomes but it is also an important mechanism in cellular processes that require access to DNA, such as transcription, replication and DNA repair. In such processes the chromatin structure is regulated to adopt distinct chromatin states ranging from a compact and inaccessible conformation (heterochromatin) to a low condensed accessible chromatin state (euchromatin). While condensed chromatin is mainly transcriptionally silent, transcription machinery and other DNA interacting factors can access DNA that is packaged into a decondensed chromatin conformation. Covalent posttranslational histone modifications (PTMs) and chromatin effector proteins are important factors for regulation and maintenance of the distinct chromatin states by either influencing the chromatin compaction state directly (e.g. by remodeling activities) or in trans by recruitment of further chromatin effector proteins.

One of the hallmarks of heterochromatin is trimethylation of lysine 9 on histone $\mathrm{H} 3$ (H3K9me3), and H3K9me3 methyl mark binding proteins are implicated in chromatin compaction and gene silencing.

In the present study, $\mathrm{H} 3 \mathrm{~K} 9 \mathrm{me} 3$ methyl mark binding proteins are investigated in $C$. elegans. LIN-61 and MBTR-1, the only two homologues of the malignant brain tumor (MBT) protein family in C. elegans were identified to interact with this repressive chromatin mark.

While MBT proteins of other organisms were reported to interact selectively with mono- and di-methylated lysine marks but with low sequence specificity, the present study shows that the $C$. elegans MBT protein LIN-61 has sequence specificity and methyl state selectivity for interaction with $\mathrm{H} 3 \mathrm{~K} 9 \mathrm{me} 2 / \mathrm{me} 3$ peptides.

By structure function analysis using $\mathrm{H} 3 \mathrm{~K} 9 \mathrm{me} 3$ peptide pull-down approaches, the present study provides first insights into the similarities and differences of the peptide binding mechanism of LIN-61 in comparison to other MBT proteins.

Phenotypic investigation of the biological functions of LIN-61 revealed that this MBT protein plays a role in negative regulation of ectopic vulval cell fate expression. In 
addition, an impact on repression of the gene lin-3 was detected. The interaction of LIN-61 with LIN-13, another factor that possess functions in vulval cell fate regulation, was identified.

Approaches to define the relevance of H3K9me3 binding for the function of LIN-61 in negative regulation of ectopic vulval cell fate expression showed that the interaction of LIN-61 with the repressive H3K9 methyl mark is essential for LIN-61 function.

In addition it was shown that besides its unique activities, LIN-61 functions redundantly with the heterochromatin protein 1 (HP1) homologue HPL-2, and the H3K9 specific histone methyl transferase MET-2. 


\section{Appendix}

\subsection{R programming for mass spectrometry analysis}

Open "R"

D1 = table 1 (e. g. output file of H3K9me0 PD lane);

D2 = table 2 (e.g. output file H3K9me3 PD lane)

> D1=read.table("/Users/nkoeste/Desktop/N_Koesters_060409_L3.txt",sep="|t",header=T)

> D2=read.table("/Users/nkoeste/Desktop/N_Koesters_060409_L4.txt",sep="|t",header=T)

> D1_D2=merge(D1,D2, by. $\mathrm{x}=1$, by. $. \mathrm{y}=1) \quad$ \#\#\# combining tables by GI number

(can also be saved as table, see below)

$>\operatorname{dim}\left(\mathrm{D} 1 \_\mathrm{D} 2\right) \quad$ \#\#\# showing dimensions of table

$>$ D2_unique $=\operatorname{subset}(\mathrm{D} 2$, is.element(prot_acc,D1_D2\$prot_acc $)==$ FALSE $)$

\#\#\#\# building a subset of GIs of all which are not in the combined table D1_D2 but in D2

$>\operatorname{dim}\left(\mathrm{D} 2 \_\right.$unique $)$

> write.table(D2_unique,file="/Users/nkoeste/Desktop/D2_unique.txt",sep="|t")

\#\#\#\# save table

\subsection{Mass spectrometry results}

\subsubsection{H3K9me3 bound C. elegans protein fraction}

\begin{tabular}{llcl}
\hline \hline GI db_id & \multicolumn{1}{c}{ protein_description } & $\begin{array}{c}\text { protein } \\
\text { score }\end{array}$ & $\begin{array}{c}\text { unique } \\
\text { peptides }\end{array}$ \\
\hline \hline gi|71999644 & hypothetical protein Y94H6A.5 & 1025 & 33 \\
\hline gi|71981040 & abnormal cell LINeage family member (lin-13) & 953 & 26 \\
\hline gi|71989520 & abnormal cell LINeage family member (lin-61) & 718 & 23 \\
\hline gi|17536159 & hypothetical protein T09A5.8 & 324 & 15 \\
\hline gi|17568549 & Heat Shock Protein family member (hsp-3) & 540 & 13 \\
\hline gi|193203511 & $\begin{array}{l}\text { MBT (Malignant Brain Tumor) Repeat containing protein } \\
\text { family member (mbtr-1) }\end{array}$ & 145 & 6 \\
\hline gi|17541180 & Adenine Nucleotide Translocator family member (ant-1.3) & 205 & 5 \\
\hline gi|2401255 & alpha-3 tubulin & 416 & 4 \\
\hline gi|25143471 & hypothetical protein Y48G8AL.5 & 81 & 3 \\
\hline \hline
\end{tabular}




\begin{tabular}{|c|c|c|c|}
\hline GI db_id & protein_description & $\begin{array}{l}\text { protein } \\
\text { score }\end{array}$ & $\begin{array}{c}\text { unique } \\
\text { peptides }\end{array}$ \\
\hline gi|17539652 & hypothetical protein F01G10.1 & 53 & 3 \\
\hline gi|17539778 & Slit-Robo GAP homolog family member (srgp-1) & 35 & 3 \\
\hline gi|71996508 & hypothetical protein T27F7.3 & 108 & 2 \\
\hline gi|17562702 & $\begin{array}{l}\text { yeast MCM (licensing factor) related family member } \\
(\mathrm{mcm}-7)\end{array}$ & 103 & 2 \\
\hline gi|17532157 & hypothetical protein $\mathrm{C} 29 \mathrm{H} 12.5$ & 95 & 2 \\
\hline gi|17532601 & Chaperonin Containing TCP-1 family member (cct-1) & 84 & 2 \\
\hline gi|17509013 & hypothetical protein T10B11.2 & 69 & 2 \\
\hline gi|17505278 & hypothetical protein B0041.8 & 69 & 2 \\
\hline gi|32563629 & hypothetical protein $\mathrm{H} 06 \mathrm{O} 01.2$ & 66 & 2 \\
\hline gi|193211092 & hypothetical protein $\mathrm{T} 25 \mathrm{C} 12.3$ & 66 & 2 \\
\hline gi|7496339 & hypothetical protein $\mathrm{C} 23 \mathrm{G} 10.4 \mathrm{a}$ & 65 & 2 \\
\hline gi|71997990 & hypothetical protein Y38E10A.22 & 63 & 2 \\
\hline gi|17509795 & Cysteinyl tRNA Synthetase family member (crs-1) & 61 & 2 \\
\hline gi|17567343 & $\begin{array}{l}\text { Propionyl Coenzyme A Carboxylase Alpha subunit family } \\
\text { member (pcca-1) }\end{array}$ & 55 & 2 \\
\hline gi|17508701 & $\begin{array}{l}\text { proteasome Regulatory Particle, ATPase-like family member } \\
\text { (rpt-5) }\end{array}$ & 53 & 2 \\
\hline gi|17564182 & Chaperonin Containing TCP-1 family member (cct-7) & 50 & 2 \\
\hline gi|17536967 & hypothetical protein Y25C1A.5 & 49 & 2 \\
\hline gi|17557798 & hypothetical protein $\mathrm{C} 10 \mathrm{G} 8.8$ & 49 & 2 \\
\hline gi|17506425 & hypothetical protein K02F2.2 & 47 & 2 \\
\hline gi|17566356 & $\begin{array}{l}\text { CAND1 (Cullin-Associated NEDD8-Dissociated protein 1) } \\
\text { homolog family member (cand-1) }\end{array}$ & 47 & 2 \\
\hline gi|193210399 & hypothetical protein T07A5.7 & 37 & 2 \\
\hline gi|133930802 & hypothetical protein F20C5.5 & 36 & 2 \\
\hline gi|17542636 & $\begin{array}{l}\text { TRAnsformer : XX animals transformed into males family } \\
\text { member (tra-3) }\end{array}$ & 36 & 2 \\
\hline gi| $\mid 17551328$ & DEgenerin Like family member (del-1) & 33 & 2 \\
\hline
\end{tabular}




\subsubsection{Common factors in bound fraction of $\mathrm{H} 3 \mathrm{~K} 9 \mathrm{me} 3 \mathrm{PD}$ and two}

\section{$\alpha L I N-61$ IPs from N2 C. elegans protein extract}

\begin{tabular}{|c|c|c|c|c|c|c|c|}
\hline \multirow{2}{*}{$\begin{array}{c}\text { GI } \\
\text { db_id }\end{array}$} & \multirow{2}{*}{$\begin{array}{c}\text { Protein } \\
\text { description }\end{array}$} & \multicolumn{2}{|c|}{$\begin{array}{c}\text { N2_H3K9me3 } \\
\text { PD }\end{array}$} & \multicolumn{2}{|c|}{ N2_IP a } & \multicolumn{2}{|c|}{ N2_IP b } \\
\hline & & $\begin{array}{c}\text { protein } \\
\text { score }\end{array}$ & $\begin{array}{c}\text { unique } \\
\text { peptides }\end{array}$ & $\begin{array}{c}\text { protein } \\
\text { score }\end{array}$ & $\begin{array}{c}\text { unique } \\
\text { peptides }\end{array}$ & $\begin{array}{c}\text { protein } \\
\text { score }\end{array}$ & $\begin{array}{l}\text { unique } \\
\text { peptides }\end{array}$ \\
\hline $\begin{array}{c}\text { gi } \\
71981 \\
040\end{array}$ & $\begin{array}{l}\text { abnormal cell } \\
\text { LINeage family } \\
\text { member (lin-13) }\end{array}$ & 953 & 26 & 129 & 3 & 56 & 1 \\
\hline $\begin{array}{c}\text { gi } \\
71989 \\
520\end{array}$ & $\begin{array}{l}\text { abnormal cell } \\
\text { LINeage family } \\
\text { member (lin-61) }\end{array}$ & 718 & 23 & 633 & 22 & 539 & 22 \\
\hline $\begin{array}{c}\text { gi } \\
17505 \\
278\end{array}$ & $\begin{array}{c}\text { hypothetical } \\
\text { protein B0041.8 }\end{array}$ & 69 & 2 & 197 & 6 & 205 & 5 \\
\hline
\end{tabular}




\section{References}

1. Avery, O.T., C.M. Macleod, and M. McCarty, Studies on the Chemical Nature of the Substance Inducing Transformation of Pneumococcal Types : Induction of Transformation by a Desoxyribonucleic Acid Fraction Isolated from Pneumococcus Type Iii. J Exp Med, 1944. 79(2): p. 137-158.

2. $\quad$ Ball, P., Portrait of a molecule. Nature, 2003. 421(6921): p. 421-2.

3. Felsenfeld, G. and M. Groudine, Controlling the double helix. Nature, 2003. 421(6921): p. 448-53.

4. Kornberg, R.D., Chromatin structure: a repeating unit of histones and DNA. Science, 1974. 184(139): p. 868-71.

5. $\quad$ Luger, K., Dynamic nucleosomes. Chromosome Res, 2006. 14(1): p. 5-16.

6. Luger, K., et al., Crystal structure of the nucleosome core particle at $2.8 \mathrm{~A}$ resolution. Nature, 1997. 389(6648): p. 251-60.

7. Arents, G. and E.N. Moudrianakis, The histone fold: a ubiquitous architectural motif utilized in DNA compaction and protein dimerization. Proc Natl Acad Sci U S A, 1995. 92(24): p. 11170-4.

8. Luger, K. and T.J. Richmond, The histone tails of the nucleosome. Curr Opin Genet Dev, 1998. 8(2): p. 140-6.

9. Robinson, P.J. and D. Rhodes, Structure of the ' $30 \mathrm{~nm}^{\prime}$ chromatin fibre: a key role for the linker histone. Curr Opin Struct Biol, 2006. 16(3): p. 336-43.

10. Thomas, J.O., Histone H1: location and role. Curr Opin Cell Biol, 1999. 11(3): p. 312-7.

11. Hansen, J.C., Conformational dynamics of the chromatin fiber in solution: determinants, mechanisms, and functions. Annu Rev Biophys Biomol Struct, 2002. 31: p. 361-92.

12. Maeshima, K. and M. Eltsov, Packaging the genome: the structure of mitotic chromosomes. J Biochem, 2008. 143(2): p. 145-53.

13. Muller, W.G., et al., Generic features of tertiary chromatin structure as detected in natural chromosomes. Mol Cell Biol, 2004. 24(21): p. 9359-70.

14. Heitz, E., Heterochromatin der Moose. Jahrbuch der Wissenschaft Botanik, 1928. 69: p. 762-818.

15. Grewal, S.I. and D. Moazed, Heterochromatin and epigenetic control of gene expression. Science, 2003. 301(5634): p. 798-802.

16. Richards, E.J. and S.C. Elgin, Epigenetic codes for heterochromatin formation and silencing: rounding up the usual suspects. Cell, 2002. 108(4): p. 489-500.

17. Perrod, S. and S.M. Gasser, Long-range silencing and position effects at telomeres and centromeres: parallels and differences. Cell Mol Life Sci, 2003. 60(11): p. 2303-18.

18. Trojer, P. and D. Reinberg, Facultative heterochromatin: is there a distinctive molecular signature? Mol Cell, 2007. 28(1): p. 1-13.

19. Kouzarides, T., Chromatin modifications and their function. Cell, 2007. 128(4): p. 693-705.

20. Campos, E.I. and D. Reinberg, Histones: annotating chromatin. Annu Rev Genet, 2009. 43: p. 559-99.

21. Talbert, P.B. and S. Henikoff, Histone variants--ancient wrap artists of the epigenome. Nat Rev Mol Cell Biol, 2010. 11(4): p. 264-75.

22. Black, B.E. and E.A. Bassett, The histone variant CENP-A and centromere specification. Curr Opin Cell Biol, 2008. 20(1): p. 91-100. 
23. Clapier, C.R. and B.R. Cairns, The biology of chromatin remodeling complexes. Annu Rev Biochem, 2009. 78: p. 273-304.

24. Bowen, N.J., et al., Mi-2/NuRD: multiple complexes for many purposes. Biochim Biophys Acta, 2004. 1677(1-3): p. 52-7.

25. Denslow, S.A. and P.A. Wade, The human Mi-2/NuRD complex and gene regulation. Oncogene, 2007. 26(37): p. 5433-8.

26. Ho, L. and G.R. Crabtree, Chromatin remodelling during development. Nature, 2010. 463(7280): p. 474-84.

27. Berger, S.L., The complex language of chromatin regulation during transcription. Nature, 2007. 447(7143): p. 407-12.

28. Sterner, D.E. and S.L. Berger, Acetylation of histones and transcriptionrelated factors. Microbiol Mol Biol Rev, 2000. 64(2): p. 435-59.

29. Zhang, Y. and D. Reinberg, Transcription regulation by histone methylation: interplay between different covalent modifications of the core histone tails. Genes Dev, 2001. 15(18): p. 2343-60.

30. Bhaumik, S.R., E. Smith, and A. Shilatifard, Covalent modifications of histones during development and disease pathogenesis. Nat Struct Mol Biol, 2007. 14(11): p. 1008-16.

31. Gelato, K.A. and W. Fischle, Role of histone modifications in defining chromatin structure and function. Biol Chem, 2008. 389(4): p. 353-63.

32. Lee, B.M. and L.C. Mahadevan, Stability of histone modifications across mammalian genomes: implications for 'epigenetic' marking. J Cell Biochem, 2009. 108(1): p. 22-34.

33. Rosenfeld, J.A., et al., Determination of enriched histone modifications in non-genic portions of the human genome. BMC Genomics, 2009. 10: p. 143.

34. Vakoc, C.R., et al., Histone H3 lysine 9 methylation and HPlgamma are associated with transcription elongation through mammalian chromatin. Mol Cell, 2005. 19(3): p. 381-91.

35. Munshi, A., et al., Histone modifications dictate specific biological readouts. $\mathrm{J}$ Genet Genomics, 2009. 36(2): p. 75-88.

36. Carrozza, M.J., et al., Histone H3 methylation by Set 2 directs deacetylation of coding regions by Rpd3S to suppress spurious intragenic transcription. Cell, 2005. 123(4): p. 581-92.

37. Keogh, M.C., et al., Cotranscriptional set2 methylation of histone H3 lysine 36 recruits a repressive Rpd3 complex. Cell, 2005. 123(4): p. 593-605.

38. Lu, X., et al., The effect of H3K79 dimethylation and H4K20 trimethylation on nucleosome and chromatin structure. Nat Struct Mol Biol, 2008. 15(10): p. 1122-4.

39. Shogren-Knaak, M., et al., Histone H4-K16 acetylation controls chromatin structure and protein interactions. Science, 2006. 311(5762): p. 844-7.

40. Ruthenburg, A.J., et al., Multivalent engagement of chromatin modifications by linked binding modules. Nat Rev Mol Cell Biol, 2007. 8(12): p. 983-94.

41. Seet, B.T., et al., Reading protein modifications with interaction domains. Nat Rev Mol Cell Biol, 2006. 7(7): p. 473-83.

42. Taverna, S.D., et al., How chromatin-binding modules interpret histone modifications: lessons from professional pocket pickers. Nat Struct Mol Biol, 2007. 14(11): p. 1025-40.

43. Mujtaba, S., L. Zeng, and M.M. Zhou, Structure and acetyl-lysine recognition of the bromodomain. Oncogene, 2007. 26(37): p. 5521-7. 
44. Zeng, L. and M.M. Zhou, Bromodomain: an acetyl-lysine binding domain. FEBS Lett, 2002. 513(1): p. 124-8.

45. Adams-Cioaba, M.A. and J. Min, Structure and function of histone methylation binding proteins. Biochem Cell Biol, 2009. 87(1): p. 93-105.

46. Ruthenburg, A.J., C.D. Allis, and J. Wysocka, Methylation of lysine 4 on histone H3: intricacy of writing and reading a single epigenetic mark. Mol Cell, 2007. 25(1): p. 15-30.

47. Maurer-Stroh, S., et al., The Tudor domain 'Royal Family': Tudor, plant Agenet, Chromo, PWWP and MBT domains. Trends Biochem Sci, 2003. 28(2): p. 69-74.

48. Jacobs, S.A. and S. Khorasanizadeh, Structure of HP1 chromodomain bound to a lysine 9-methylated histone H3 tail. Science, 2002. 295(5562): p. 2080-3.

49. Li, H., et al., Structural basis for lower lysine methylation state-specific readout by $M B T$ repeats of $L 3 M B T L 1$ and an engineered PHD finger. Mol Cell, 2007. 28(4): p. 677-91.

50. Jacobs, S.A., et al., Specificity of the HPI chromo domain for the methylated $N$-terminus of histone H3. EMBO J, 2001. 20(18): p. 5232-41.

51. Min, J., Y. Zhang, and R.M. Xu, Structural basis for specific binding of Polycomb chromodomain to histone H3 methylated at Lys 27. Genes Dev, 2003. 17(15): p. 1823-8.

52. Fischle, W., et al., Molecular basis for the discrimination of repressive methyl-lysine marks in histone H3 by Polycomb and HP1 chromodomains. Genes Dev, 2003. 17(15): p. 1870-81.

53. Bonasio, R., E. Lecona, and D. Reinberg, MBT domain proteins in development and disease. Seminars in Cell \& Developmental Biology, 2010. 21(2): p. 221-230.

54. Qiao, F. and J.U. Bowie, The many faces of SAM. Sci STKE, 2005. 2005(286): p. re7.

55. Grimm, C., et al., Structural and functional analyses of methyl-lysine binding by the malignant brain tumour repeat protein Sex comb on midleg. EMBO Rep, 2007. 8(11): p. 1031-7.

56. Grimm, C., et al., Molecular recognition of histone lysine methylation by the Polycomb group repressor dSfmbt. EMBO J, 2009. 28(13): p. 1965-77.

57. Guo, Y., et al., Methylation-state-specific recognition of histones by the MBT repeat protein L3MBTL2. Nucleic Acids Res, 2009. 37(7): p. 2204-10.

58. Kalakonda, N., et al., Histone H4 lysine 20 monomethylation promotes transcriptional repression by L3MBTL1. Oncogene, 2008. 27(31): p. 4293304.

59. Min, J., et al., L3MBTL1 recognition of mono- and dimethylated histones. Nat Struct Mol Biol, 2007. 14(12): p. 1229-30.

60. Santiveri, C.M., et al., The malignant brain tumor repeats of human SCML2 bind to peptides containing monomethylated lysine. J Mol Biol, 2008. 382(5): p. 1107-12.

61. Wang, W.K., et al., Malignant brain tumor repeats: a three-leaved propeller architecture with ligand/peptide binding pockets. Structure, 2003. 11(7): p. 775-89.

62. Sathyamurthy, A., et al., Crystal structure of the malignant brain tumor (MBT) repeats in Sex Comb on Midleg-like 2 (SCML2). J Biol Chem, 2003. 278(47): p. 46968-73. 
63. Eryilmaz, J., et al., Structural studies of a four-MBT repeat protein MBTD1. PLoS One, 2009. 4(10): p. e7274.

64. Wu, S., R.C. Trievel, and J.C. Rice, Human SFMBT is a transcriptional repressor protein that selectively binds the N-terminal tail of histone H3. FEBS Lett, 2007. 581(17): p. 3289-96.

65. Gateff, E., T. Loffler, and J. Wismar, A temperature-sensitive brain tumor suppressor mutation of Drosophila melanogaster: developmental studies and molecular localization of the gene. Mech Dev, 1993. 41(1): p. 15-31.

66. Yohn, C.B., et al., l(3)malignant brain tumor and three novel genes are required for Drosophila germ-cell formation. Genetics, 2003. 165(4): p. 1889900.

67. Koga, H., et al., A human homolog of Drosophila lethal(3)malignant brain tumor (l(3)mbt) protein associates with condensed mitotic chromosomes. Oncogene, 1999. 18(26): p. 3799-809.

68. Scharf, A.N., et al., Monomethylation of lysine 20 on histone H4 facilitates chromatin maturation. Mol Cell Biol, 2009. 29(1): p. 57-67.

69. Boccuni, P., et al., The human L(3)MBT polycomb group protein is a transcriptional repressor and interacts physically and functionally with TEL (ETV6). J Biol Chem, 2003. 278(17): p. 15412-20.

70. Lu, J., et al., A genome-wide RNA interference screen identifies putative chromatin regulators essential for E2F repression. Proc Natl Acad Sci U S A, 2007. 104(22): p. 9381-6.

71. Trojer, P., et al., L3MBTL1, a histone-methylation-dependent chromatin lock. Cell, 2007. 129(5): p. 915-28.

72. Breen, T.R. and I.M. Duncan, Maternal expression of genes that regulate the bithorax complex of Drosophila melanogaster. Dev Biol, 1986. 118(2): p. 442-56.

73. Klymenko, T., et al., A Polycomb group protein complex with sequencespecific DNA-binding and selective methyl-lysine-binding activities. Genes Dev, 2006. 20(9): p. 1110-22.

74. Shao, Z., et al., Stabilization of chromatin structure by PRC1, a Polycomb complex. Cell, 1999. 98(1): p. 37-46.

75. Saurin, A.J., et al., A Drosophila Polycomb group complex includes Zeste and dTAFII proteins. Nature, 2001. 412(6847): p. 655-60.

76. Stielow, B., et al., Identification of SUMO-dependent chromatin-associated transcriptional repression components by a genome-wide RNAi screen. Mol Cell, 2008. 29(6): p. 742-54.

77. Cui, M. and M. Han, Roles of chromatin factors in C. elegans development. WormBook, 2007: p. 1-16.

78. Kolasinska-Zwierz, P., et al., Differential chromatin marking of introns and expressed exons by H3K36me3. Nat Genet, 2009. 41(3): p. 376-81.

79. Schaner, C.E. and W.G. Kelly, Germline chromatin. WormBook, 2006: p. 114.

80. Fay, D.S. and J. Yochem, The SynMuv genes of Caenorhabditis elegans in vulval development and beyond. Dev Biol, 2007. 306(1): p. 1-9.

81. Sternberg, P.W., Vulval development. WormBook, 2005: p. 1-28.

82. Ferguson, E.L., P.W. Sternberg, and H.R. Horvitz, A genetic pathway for the specification of the vulval cell lineages of Caenorhabditis elegans. Nature, 1987. 326(6110): p. 259-67. 
83. Moghal, N. and P.W. Sternberg, The epidermal growth factor system in Caenorhabditis elegans. Exp Cell Res, 2003. 284(1): p. 150-9.

84. Ferguson, E.L. and H.R. Horvitz, Identification and characterization of 22 genes that affect the vulval cell lineages of the nematode Caenorhabditis elegans. Genetics, 1985. 110(1): p. 17-72.

85. Horvitz, H.R. and J.E. Sulston, Isolation and genetic characterization of celllineage mutants of the nematode Caenorhabditis elegans. Genetics, 1980. 96(2): p. 435-54.

86. Hill, R.J. and P.W. Sternberg, The gene lin-3 encodes an inductive signal for vulval development in C. elegans. Nature, 1992. 358(6386): p. 470-6.

87. Beitel, G.J., S.G. Clark, and H.R. Horvitz, Caenorhabditis elegans ras gene let-60 acts as a switch in the pathway of vulval induction. Nature, 1990. 348(6301): p. 503-9.

88. Ferguson, E.L. and H.R. Horvitz, The multivulva phenotype of certain Caenorhabditis elegans mutants results from defects in two functionally redundant pathways. Genetics, 1989. 123(1): p. 109-21.

89. Cui, M., et al., SynMuv genes redundantly inhibit lin-3/EGF expression to prevent inappropriate vulval induction in C. elegans. Dev Cell, 2006. 10(5): p. 667-72.

90. Huang, L.S., P. Tzou, and P.W. Sternberg, The lin-15 locus encodes two negative regulators of Caenorhabditis elegans vulval development. Mol Biol Cell, 1994. 5(4): p. 395-411.

91. Herman, R.K. and E.M. Hedgecock, Limitation of the size of the vulval primordium of Caenorhabditis elegans by lin-15 expression in surrounding hypodermis. Nature, 1990. 348(6297): p. 169-71.

92. Myers, T.R. and I. Greenwald, lin-35 Rb acts in the major hypodermis to oppose ras-mediated vulval induction in C. elegans. Dev Cell, 2005. 8(1): p. 117-23.

93. Ceol, C.J. and H.R. Horvitz, dpl-1 DP and efl-1 E2F act with lin-35 Rb to antagonize Ras signaling in C. elegans vulval development. Mol Cell, 2001. 7(3): p. 461-73.

94. Lu, X. and H.R. Horvitz, lin-35 and lin-53, two genes that antagonize a $C$. elegans Ras pathway, encode proteins similar to $R b$ and its binding protein RbAp48. Cell, 1998. 95(7): p. 981-91.

95. Frolov, M.V. and N.J. Dyson, Molecular mechanisms of E2F-dependent activation and pRB-mediated repression. J Cell Sci, 2004. 117(Pt 11): p. 2173-81.

96. Polager, S. and D. Ginsberg, E2F - at the crossroads of life and death. Trends Cell Biol, 2008. 18(11): p. 528-35.

97. Korenjak, M., et al., Native E2F/RBF complexes contain Myb-interacting proteins and repress transcription of developmentally controlled E2F target genes. Cell, 2004. 119(2): p. 181-93.

98. Lewis, P.W., et al., Identification of a Drosophila Myb-E2F2/RBF transcriptional repressor complex. Genes Dev, 2004. 18(23): p. 2929-40.

99. Harrison, M.M., et al., Some C. elegans class B synthetic multivulva proteins encode a conserved LIN-35 Rb-containing complex distinct from a NuRD-like complex. Proc Natl Acad Sci U S A, 2006. 103(45): p. 16782-7.

100. Harrison, M.M., X. Lu, and H.R. Horvitz, LIN-61, one of two Caenorhabditis elegans malignant-brain-tumor-repeat-containing proteins, acts with the DRM 
and NuRD-like protein complexes in vulval development but not in certain other biological processes. Genetics, 2007. 176(1): p. 255-71.

101. Poulin, G., et al., Chromatin regulation and sumoylation in the inhibition of Ras-induced vulval development in Caenorhabditis elegans. EMBO J, 2005. 24(14): p. 2613-23.

102. von Zelewsky, T., et al., The C. elegans Mi-2 chromatin-remodelling proteins function in vulval cell fate determination. Development, 2000. 127(24): p. 5277-84.

103. Unhavaithaya, Y., et al., MEP-1 and a homolog of the NURD complex component Mi-2 act together to maintain germline-soma distinctions in $C$. elegans. Cell, 2002. 111(7): p. 991-1002.

104. Kunert, N., et al., dMec: a novel Mi-2 chromatin remodelling complex involved in transcriptional repression. EMBO J, 2009. 28(5): p. 533-44.

105. Ceol, C.J. and H.R. Horvitz, A new class of C. elegans synMuv genes implicates a Tip60/NuA4-like HAT complex as a negative regulator of Ras signaling. Dev Cell, 2004. 6(4): p. 563-76.

106. Galarneau, L., et al., Multiple links between the NuA4 histone acetyltransferase complex and epigenetic control of transcription. Mol Cell, 2000. 5(6): p. 927-37.

107. Ikura, T., et al., Involvement of the TIP60 histone acetylase complex in DNA repair and apoptosis. Cell, 2000. 102(4): p. 463-73.

108. Niida, H., et al., Essential role of Tip60-dependent recruitment of ribonucleotide reductase at DNA damage sites in DNA repair during G1 phase. Genes Dev, 2010. 24(4): p. 333-8.

109. Sun, Y., et al., Histone H3 methylation links DNA damage detection to activation of the tumour suppressor Tip60. Nat Cell Biol, 2009. 11(11): p. 1376-82.

110. Qi, D., et al., Drosophila Reptin and other TIP60 complex components promote generation of silent chromatin. Genetics, 2006. 174(1): p. 241-51.

111. Taubert, S., et al., E2F-dependent histone acetylation and recruitment of the Tip60 acetyltransferase complex to chromatin in late G1. Mol Cell Biol, 2004. 24(10): p. 4546-56.

112. Andersen, E.C. and H.R. Horvitz, Two C. elegans histone methyltransferases repress lin-3 EGF transcription to inhibit vulval development. Development, 2007. 134(16): p. 2991-9.

113. Couteau, F., et al., A heterochromatin protein 1 homologue in Caenorhabditis elegans acts in germline and vulval development. EMBO Rep, 2002. 3(3): p. 235-41.

114. Schott, S., et al., Unique and redundant functions of C. elegans HP1 proteins in post-embryonic development. Dev Biol, 2006. 298(1): p. 176-87.

115. Schott, S., et a1., HPL-2/HP1 prevents inappropriate vulval induction in Caenorhabditis elegans by acting in both HYP7 and vulval precursor cells. Genetics, 2009. 181(2): p. 797-801.

116. Coustham, V., et al., The C. elegans HP1 homologue HPL-2 and the LIN-13 zinc finger protein form a complex implicated in vulval development. Dev Biol, 2006. 297(2): p. 308-22.

117. Wirth, M., et al., HIS-24 linker histone and SIR-2.1 deacetylase induce H3K27me3 in the Caenorhabditis elegans germ line. Mol Cell Biol, 2009. 29(13): p. 3700-9. 
118. Melendez, A. and I. Greenwald, Caenorhabditis elegans lin-13, a member of the LIN-35 Rb class of genes involved in vulval development, encodes a protein with zinc fingers and an LXCXE motif. Genetics, 2000. 155(3): p. 1127-37.

119. Andersen, E.C., A.M. Saffer, and H.R. Horvitz, Multiple levels of redundant processes inhibit Caenorhabditis elegans vulval cell fates. Genetics, 2008. 179(4): p. 2001-12.

120. Dufourcq, P., et al., Functional requirement for histone deacetylase 1 in Caenorhabditis elegans gonadogenesis. Mol Cell Biol, 2002. 22(9): p. 302434.

121. Garbe, D., J.B. Doto, and M.V. Sundaram, Caenorhabditis elegans lin-35/Rb, efl-1/E2F and other synthetic multivulva genes negatively regulate the anaphase-promoting complex gene mat-3/APC8. Genetics, 2004. 167(2): p. 663-72.

122. Wang, D., et al., Somatic misexpression of germline P granules and enhanced RNA interference in retinoblastoma pathway mutants. Nature, 2005. 436(7050): p. 593-7.

123. Mello, C.C., et al., Efficient gene transfer in C.elegans: extrachromosomal maintenance and integration of transforming sequences. EMBO J, 1991. 10(12): p. 3959-70.

124. Brenner, S., The genetics of Caenorhabditis elegans. Genetics, 1974. 77(1): p. 71-94.

125. New England Biolabs, I., Catalog and technical reference. 2007/200.

126. Sambrook, J. and D. Russel, Molecular Cloning. A laboratory Manual. 3rd ed. 2001: Cold Spring Harbor, New York: Cold Spring Harbour press.

127. Laemmli, U.K., Cleavage of structural proteins during the assembly of the head of bacteriophage T4. Nature, 1970. 227(5259): p. 680-5.

128. Gallagher, S.R., One-dimensional SDS gel electrophoresis of proteins. Curr Protoc Immunol, 2006. Chapter 8: p. Unit 84.

129. Shevchenko, A., et al., Mass spectrometric sequencing of proteins silverstained polyacrylamide gels. Anal Chem, 1996. 68(5): p. 850-8.

130. QIAexpressionist, T., A handbook for high level expression and purification of 6xHis-tagged proteins.

131. Wysocka, J., Identifying novel proteins recognizing histone modifications using peptide pull-down assay. Methods, 2006. 40(4): p. 339-43.

132. Stiernagle, T., Maintenance of C. elegans. WormBook, 2006: p. 1-11.

133. Lewis, J.A. and J.F. Flemming, Basic culture methods, in Methods in Cell Biology Caenorhabditis elegans Modern Biological Analysis of an Organism, E.H.F.a.S. D.C., Editor. 1995.

134. Cheeseman, I.M., et al., A conserved protein network controls assembly of the outer kinetochore and its ability to sustain tension. Genes Dev, 2004. 18(18): p. 2255-68.

135. Duerr, J.S., Immunohistochemistry. WormBook, 2006: p. 1-61.

136. Livak, K.J. and T.D. Schmittgen, Analysis of relative gene expression data using real-time quantitative $P C R$ and the 2(-Delta Delta $C(T)$ ) Method. Methods, 2001. 25(4): p. 402-8.

137. Kamath, R.S. and J. Ahringer, Genome-wide RNAi screening in Caenorhabditis elegans. Methods, 2003. 30(4): p. 313-21.

138. Stinchcomb, D.T., et al., Extrachromosomal DNA transformation of Caenorhabditis elegans. Mol Cell Biol, 1985. 5(12): p. 3484-96. 
139. Kramer, J.M., et al., The Caenorhabditis elegans rol-6 gene, which interacts with the sqt-1 collagen gene to determine organismal morphology, encodes a collagen. Mol Cell Biol, 1990. 10(5): p. 2081-9.

140. Mello, C. and A. Fire, DNA transformation in Methods Cell Biology. 1995. p. 451-482.

141. Hodgkin, J. and T.M. Barnes, More is not better: brood size and population growth in a self-fertilizing nematode. Proc Biol Sci, 1991. 246(1315): p. 1924.

142. Sutphin, G.L. and M. Kaeberlein, Measuring Caenorhabditis elegans life span on solid media. J Vis Exp, 2009(27).

143. Apfeld, J. and C. Kenyon, Cell nonautonomy of C. elegans daf-2 function in the regulation of diapause and life span. Cell, 1998. 95(2): p. 199-210.

144. Simonet, T., et al., Antagonistic functions of SET-2/SET1 and HPL/HP1 proteins in C. elegans development. Dev Biol, 2007. 312(1): p. 367-83.

145. Fischle, W., et al., Specificity of the chromodomain Y chromosome family of chromodomains for lysine-methylated ARK(S/T) motifs. J Biol Chem, 2008. 283(28): p. 19626-35.

146. Franz, H., et al., Multimerization and $H 3 \mathrm{~K} 9 m e 3$ binding are required for CDYL1b heterochromatin association. J Biol Chem, 2009. 284(50): p. 3504959.

147. Bouazoune, K., et al., The dMi-2 chromodomains are DNA binding modules important for ATP-dependent nucleosome mobilization. EMBO J, 2002. 21(10): p. 2430-40.

148. Akhtar, A., D. Zink, and P.B. Becker, Chromodomains are protein-RNA interaction modules. Nature, 2000. 407(6802): p. 405-9.

149. Huang, Y., et al., Recognition of histone H3 lysine-4 methylation by the double tudor domain of JMJD2A. Science, 2006. 312(5774): p. 748-51.

150. Botuyan, M.V., et al., Structural basis for the methylation state-specific recognition of histone H4-K20 by 53BP1 and Crb2 in DNA repair. Cell, 2006. 127(7): p. 1361-73.

151. Collins, R.E., et al., The ankyrin repeats of G9a and GLP histone methyltransferases are mono- and dimethyllysine binding modules. Nat Struct Mol Biol, 2008. 15(3): p. 245-50.

152. Hubbard, E.J. and D. Greenstein, The Caenorhabditis elegans gonad: a test tube for cell and developmental biology. Dev Dyn, 2000. 218(1): p. 2-22. 


\section{Acknowledgements}

First and foremost, I would like to thank my supervisor, Dr. Wolfgang Fischle, for giving me the opportunity to work in his lab, his continuous support and guidance. I am grateful for all the fruitful discussions, encouraging ideas and his motivating supervision.

I am very grateful to my $\mathrm{PhD}$ committee members Prof. Dr. Sigrid Hoyer-Fender and Prof. Dr. Herbert Jäckle for their interest in my project and for all their helpful suggestions and fruitful discussions.

I am very grateful to Dr. Monika Jedrusik-Bode for her constant help and fruitful discussions.

Many, many thanks to all lab members of the Fischle group for the pleasant time throughout my thesis, for the constant help and advise.

I want to thank the GGNB program for the constant support, informative method courses and inspiring retreats.

I am grateful to Dr. Henning Urlaub and his group for their excellent mass spectrometry work and for helpful advises and motivating explanations.

I am grateful to Dr. Dieter Klopfenstein and his group for giving me the permission to perform C. elegans microinjection in their lab, all their help and advices.

I want to thank Dr. Henrik Bringmann and his group for giving me the permission to take DIC images of C. elegans and their help.

I thank Prof. Dr. Horvitz, Prof. Dr. Greenwald, Dr. Palladino, the people from the Caenorhabditis genetics Center and the people from the Mitani Laboratory for sharing reagents. 
Many thanks to Haixia Ding, Franziska Paap, Jennifer Bals and Claudia Fahlbusch for their great help in preparing media and buffers and for pouring so many plates.

I am very thankfull to Kathy Gelato and Lena Hyatt for reading and correcting the manuscript.

Especially, I want to thank Henriette Franz, Martina Wirth, Szabolcs Sörös and Kerstin Mosch for their great help in many many steps of my thesis, their friendship and all the nice shared time vom Frühstück bis zum Feierabend-Bierle!

Lastly, I want to thank my family for always being there for me, their continuous support, their help!! 


\section{Curriculum vitae}

Personal data

Name:

Nora Köster-Eiserfunke

Date of birth:

26.08.1979

Place of birth:

Aachen, Germany

Nationality:

German

\section{Schooling}

$1986-1988$

Grundschule Gut Kullen, Aachen (elementary school)

$1988-1990$

Grundschule Werste, Bad Oeynhausen (elementary school)

1990 - 1999

Immanuel-Kant-Gymnasium Bad Oeynhausen

05/1999

Abitur

\section{Studies}

10/1999-12/2004 Biochemistry

Martin-Luther-University Halle-Wittenberg, Halle (Saale)

Diploma in Biochemistry

Diploma thesis: "Analysis of putative phosphoproteins in transgenic Arabidopsis-plants with enhanced MAPkinase-3 and -6 activity"

Since $06 / 2006$

GGNB "Molecular Biology of Development and Interaction between Organisms"

Georg-August-University Göttingen

$\mathrm{PhD}$ studies

Thesis: "Characterization of LIN-61 methyl mark binding and its function in C. elegans vulva development"

\section{Work Experience}

04/2005 - 09/2005 Research Assistant

School of Biological Sciences, University of Aberdeen

\section{Stipend}

$2006-2008$

Max-Planck-Institute stipend 